\title{
MODIFICATION OF POLYPROPYLENE MEMBRANES AND FILMS BY CATALYTIC OZONATION
}

\author{
By \\ Hong Bin $\mathrm{Gu}$ \\ (M.A.Sc., Ryerson University, Toronto, Ontario, Canada, 2008)
}

(B.Sc, Wuhan University, Wuhan City, Hubei Province, P. R. China, 1988)

\author{
A dissertation \\ Presented to Ryerson University \\ In partial fulfillment of the \\ Requirements for the degree of \\ Doctor of Philosophy \\ In the program of \\ Chemical Engineering
}

Toronto, Ontario, Canada, 2014

CHong Bin Gu 2014 


\section{AUTHOR'S DECLARATION}

I hereby declare that I am the sole author of this dissertation. This is a true copy of the dissertation, including any required final revisions, as accepted by my examiners.

I authorize Ryerson University to lend this dissertation to other institutions or individuals for the purpose of scholarly research.

I further authorize Ryerson University to reproduce this dissertation by photocopying or by other means, in total or parts, at the request of other institutions or individuals for the purpose of scholarly research.

I understand that my dissertation may be made electronically available to the public.

\section{Hong Bir Ga}




\section{BORROWER'S PAGE}

Ryerson University requires the signatures of all persons using or photocopying this thesis.

Please sign below, and give address and date.

\begin{tabular}{|c|c|c|c|}
\hline Name of Borrowers & Date & Address & Signature \\
\hline & & & \\
\hline & & & \\
\hline & & & \\
\hline & & & \\
\hline & & & \\
\hline & & & \\
\hline & & & \\
\hline & & & \\
\hline & & & \\
\hline & & & \\
\hline & & & \\
\hline & & & \\
\hline & & & \\
\hline & & & \\
\hline & & & \\
\hline
\end{tabular}




\section{ABSTRACT \\ Hydrophilic Modification of Polypropylene Membranes and Films by Catalytic Ozonation \\ Hong Bin Gu \\ Doctor of Philosophy}

Department of Chemical Engineering, Ryerson University, Toronto, 2014

Commercial polypropylene microfiltration membranes (PPMM) and biaxial oriented polypropylene (BOPP) films were ozonated in aqueous and gaseous media, respectively, followed by graft polymerization of acryl amide (AAm), hydroxyethyl methacrylate (HEMA), and polyethylene glycol (PEG) to improve their surface hydrophilicity. The efficiency of ozonation conducted in the gaseous and aqueous phases was compared, the gaseous phase ozonation was found slightly more effective in generating peroxide groups, while the aqueous phase ozonation was found more effective in grafting polymerization. Scavengers were added to the aqueous phase ozonation, results indicated that both the radical groups and the molecule ozone contributed to the peroxide generation. The free radical groups contributed maximum 25\% and 32\% for ozonation of PPMM and BOPP, respectively, and the molecule ozone contributed the percentage remaining. Results also showed that the concentration of peroxides generated on the surfaces of PPMM or BOPP increased with the applied ozone dose and ozonation time in both phases.

Copper sulfate hydrate $\left(\mathrm{CuSO}_{4} \cdot 5 \mathrm{H}_{2} \mathrm{O}\right)$ and ferric chloride hydrate $\left(\mathrm{FeCl}_{3} \cdot 6 \mathrm{H}_{2} \mathrm{O}\right)$ were added in the aqueous phase ozonation as homogenous catalysts, results showed that the peroxide generation rate of PPMM and BOPP was improved comparing to that of ozonation without catalyst. The peroxide generation of PPMM showed 17\% increase by adding copper catalyst, and $16 \%$ increase in peroxide generation was observed in ferric catalyzed ozonation of BOPP. The mechanism of the aqueous phase ozonation was investigated, along with that of catalytic ozonation. An enhanced radical process was found for catalytic ozonation in this study.

The hydrophilicity of PPMM and BOPP was improved by graft polymerization of 
AAm, HEMA and PEG initiated by the peroxides. The aqueous phase ozonation was found more effective in grafting. A washing test was conducted using distilled water blending with $10 \%$ isopropyl alcohol. When the ozonated membranes and films were washed and compared to the non-washed ones, it was found that the gaseous phase ozonated PPMM or BOPP lost more peroxides than their aqueous phase counterpart after washing. The washing tests showed that the aqueous phase ozonation could induce a better graft polymerization, because part of the tested peroxides from the gaseous ozonation was washed away in the cleaning and grafting process.

The improved hydrophilicity of PPMM was indicated by the contact angle reduction from $129^{\circ}$ to $91^{\circ}$ for AAm grafting; from $126^{\circ}$ to $74^{\circ}$ for HEMA grafting; and from $126^{\circ}$ to $88^{\circ}$ for PEG grafting; Fourier Transform Infrared (FTIR) measurements showed additional peaks of functional groups, such as amine $(\mathrm{N}-\mathrm{H})$ and amide $(-\mathrm{N}-\mathrm{C}=\mathrm{O})$ functional groups from the grafted AAm $\left(\mathrm{CH}_{2}=\mathrm{CH}-\mathrm{CO}-\mathrm{NH}_{2}\right)$; and the Scanning Electron Microscope (SEM) images confirmed amorphicity changes of the graft polymerization. X-ray Diffraction (XRD) diffractogram revealed the crystallinity changes of ozonated and grafted PPMM. Bovine serum albumin (BSA) was used to test the filtration performance of virgin and grafted membranes, the filtration tests demonstrated the improvement in anti-fouling effect of the modified PPMM; and the SEM images of the fouled and washed membranes revealed the pore blockage and recovering on the surface.

The hydrophilicity of the grafted BOPP was also improved, indicated by the contact angle reduction of AAm grafted film from $80^{\circ}$ to $56^{\circ}$. The FTIR showed additional peaks of $\mathrm{N}-\mathrm{H}$ and $-\mathrm{N}-\mathrm{C}=\mathrm{O}$ functional groups of grafted AAm. SEM images indicated amorphicity changes of the graft polymerization. The film modified by the aqueous phase ozonation showed its advantages of better graft polymerization, hydrophilicity, and protein adsorption.

The results of this study positively impacted the industrial using of PPMM to elongate its duration time of filtration, and improved the applications of BOPP in biomedical areas.

Keywords: membrane, film, hydrophilic, ozonation, aqueous phase, copper and ferric catalyst, mechanism, graft polymerization, AAm, HEMA, PEG, BSA, filtration, FTIR, SEM, XRD 


\section{ACKNOWLEDGEMENTS}

I would like to express my deep gratitude to my supervisors, Dr. Jiangning Wu and Dr. Philip Chan for their attentive and comprehensive guidance, invaluable advice and support throughout the research to accomplish this thesis.

I would like to show my deep appreciation to the faculty members and technologists in Chemical Engineering Department of Ryerson University for the facilities and assistance provided during my program in the university.

I am grateful for financial support of Natural Science and Engineering Research Council of Canada (NSERC) for funding this research. 


\section{DEDICATION}

Attribute to my father and mother for the bestowal of a beautiful life to me, attribute to my family members who support me all the time, tolerant of my mood variation and unhappiness when I faced difficulty, especially at the hard time when I was sick and did a severe cardinal surgery during the year 2012; and for those unconditional love, consistent encouragement and forgiveness throughout my whole life, wherever I am. 


\section{TABLE OF CONTENTS}

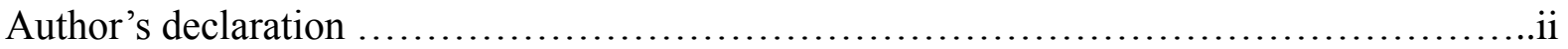

Borrower's page..................................................................ii

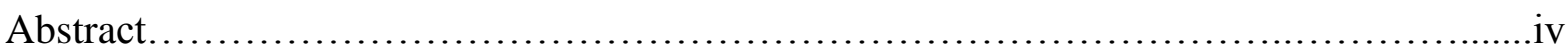

Acknowledgements.................................................................

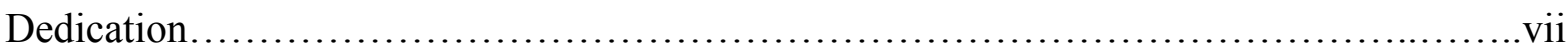

Table of Contents....................................................................

List of Tables......................................................................

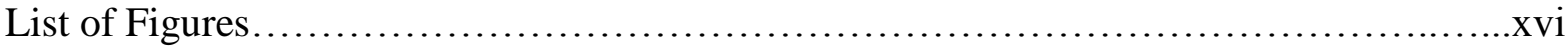

\section{Chapter 1}

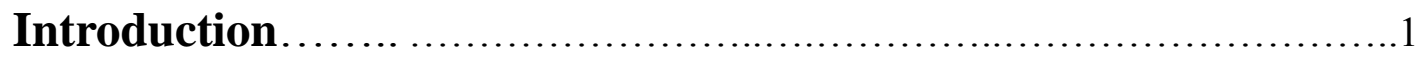

\section{Chapter 2}

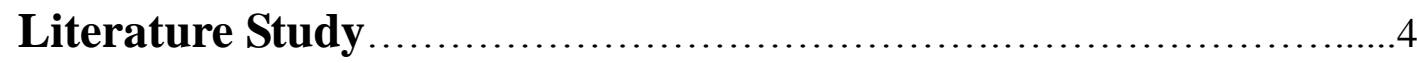

2.1 Membrane, films and their modifications........................................ 4

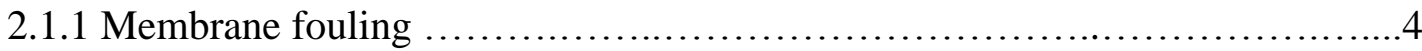

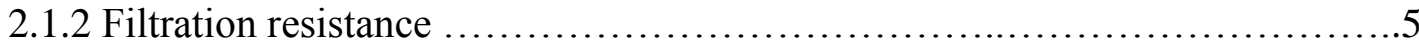

2.1.3 Membrane fouling .....................................................

2.1.4 Modifications to improve membrane anti-fouling..........................

2.1.5 Polymeric films and modifications..................................

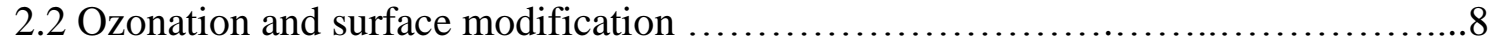

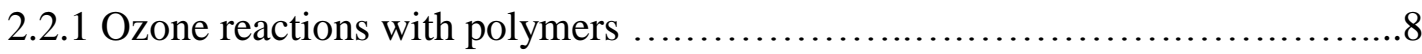

2.2.2 Peroxide Generation and Decomposition on Polymer Surface..................9

2.2.2.1 Redox decomposition of peroxide ...................................9

2.2.2.2 Thermal decomposition of peroxide ...............................10

2.2.3 Graft copolymerization............................................. 10 
2.3 Ozone properties......................................................11

2.3.1 Properties of ozone and comparison with oxygen........................11

2.3.2 Decomposition of ozone in water and $\mathrm{pH}$ effect. ..........................13

2.3.2.1 Effect of hydroxide ions $\mathrm{OH}^{-}$on ozone decomposition...................13

2.3.2.2 Equations in ozone decomposition.................................16

2.4 Mass Transfer............................................................ 20

2.4.1 Mass transfer of ozone in two phases....................................20

2.4.1.1 Equilibrium concentration for ozone ..................................20

2.4.1.2 Mass transfer with simultaneous chemical reactions.....................21

2.4.1.3 Determination of mass transfer coefficients of continuous-flow mode....22

2.4.2 Determination of the gas-liquid dispersion parameters $\left(\mathrm{d}_{32}, \varepsilon \mathrm{G}\right.$, a and $\left.\mathrm{U}_{\mathrm{S}}\right) \ldots .23$

2.4.3. Simultaneous determination of mass transfer parameters $\left(\mathrm{k}_{\mathrm{La}}, \mathrm{k}_{\mathrm{C}}\right.$ and $\left.\mathrm{m}\right) \ldots \ldots .24$

2.5 Ozonation kinetics and scavengers......................................... 26

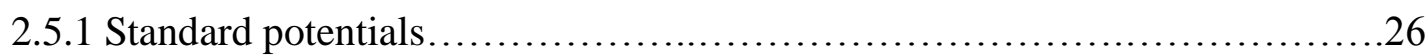

2.5.2 Reaction kinetics of ozone in pure water...............................27

2.5.2.1 Initiation step. ...............................................27

2.5.2.2 Propagation step. ................................................28

2.5.2.3 Termination step. .............................................29

2.5.3 Other hypothesis of ozonation in the aqueous phase.......................30

2.5.4 Mechanism about peroxide generation of ozonation on polyethylene..........33

2.6 Kinetics of membrane flux decline......................................... 34

2.6.1 Gas and liquid fouling model. ...................................... 34

2.6.2 Dead-end filtration fouling model...................................... 35

2.6.3 Combined pore blockage and cake filtration fouling model...................36

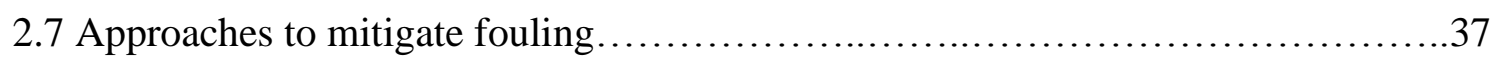

2.7.1 Surface modification............................................... 37

2.7.2 Chemical schematic of graft polymerization..............................37

2.7.3 Degree of grafting and grafting efficiency.............................. 38

2.7.4 Effect of grafting on surface roughness and permeability....................39

2.8 Permeate flux............................................................. 39 
2.9 Other researchers' work of membrane anti-fouling and modification. 40

2.9.1 Hydrophilic modification of polypropylene microfiltration membranes by ozone -induced graft polymerization.....................................40

2.9.2 Membrane fouling reduction by backpulsing and surface modification........42

2.9.3 Polyolefins reacted with $\mathrm{O}_{3}$ in aqueous phase..........................43

2.9.4 BOPP reacted with $\mathrm{O}_{3}$ in gaseous and aqueous phases...................47

2.9.5 List of other membrane anti-fouling modification......................47

\section{Chapter 3}

Experimental Methodology ..........................................49

3.1 Materials...................................................................... 49

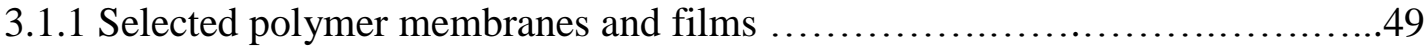

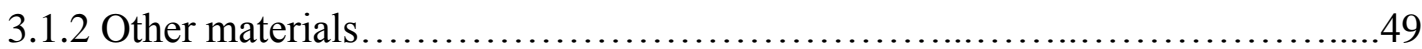

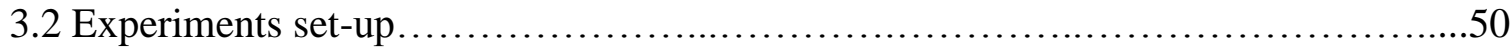

3.2.1 Experiment set-up for membrane and film ozonation......................50

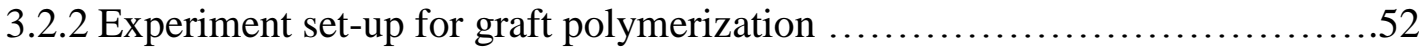

3.3. Experimental procedure.................................................. 53

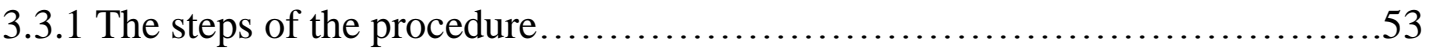

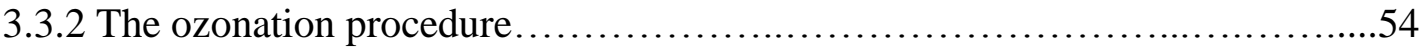

3.3.3 The graft procedure .................................................56

3.3.4 The filtration test and protein adsorption procedure $\ldots \ldots \ldots \ldots \ldots \ldots \ldots \ldots \ldots .56$

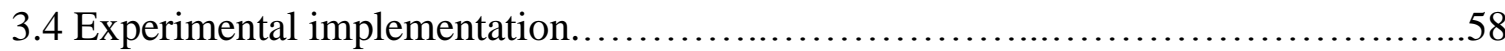

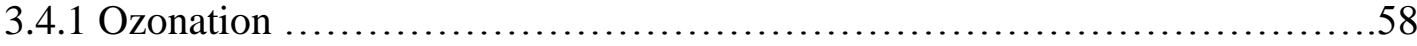

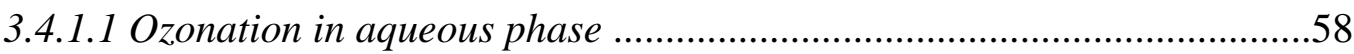

3.4.1.2 Ozonation in gaseous phase .................................................................58

3.4.1.3 Investigation of the ozone dose ..................................58

3.4.1.4 The study of the effect of varying $\mathrm{pH}$ value in aqueous phase reactions..59

3.4.1.5 Scavenger study .............................................5 
3.4.1.6 Washing test..................................................59

3.4.1.7 Aging study...............................................60

3.4.2 Catalysts in aqueous phase ozonation study...........................60

3.4.2.1 $\mathrm{Fe}^{3+}$ ion used as catalyst........................................60

3.4.2.2 $\mathrm{Cu}^{2+}$ ion used as catalyst .......................................60

3.4.3 Investigative tests of the mechanism on catalyst $\mathrm{Cu}^{2+} \ldots \ldots \ldots \ldots \ldots \ldots \ldots \ldots . . .61$

3.4.3.1 $\mathrm{Cu}^{2+}$ catalytic ozonation applied at a low temperature. ${ }^{+}$...............61

3.4.3.2 Scavenger tests in $\mathrm{Cu}^{2+}$ catalytic ozonation ..........................61

3.4.3.3 The effect of the anion $\mathrm{SO}_{4}{ }^{2-}$ on $\mathrm{DW}$ ozonation......................61

3.4.3.4 Investigation of dissolved ozone in DW undergoing $\mathrm{Cu}^{2+}$ catalytic

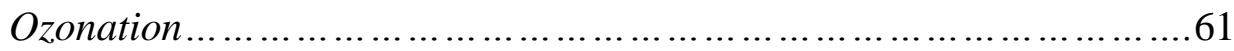

3.4.3.5 Hydroxyl radical capture by Nitrobenzene (NB) applied to ozonation with or without $\mathrm{Cu}^{2+}$ catalyst........................................61

3.4.4 Graft polymerization of the specific film under optimized condition...........62

3.4.4.1 AAm graft polymerization of the specific film........................62

3.4.4.2 PEG graft polymerization of the specific film.........................66

3.4.4.3 HEMA graft polymerization of the specific film ........................63

3.4.5 Dead-end filtration test............................................63

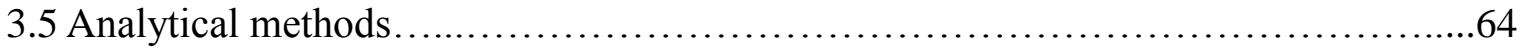

3.5.1 Standard metric iodide method............................................64

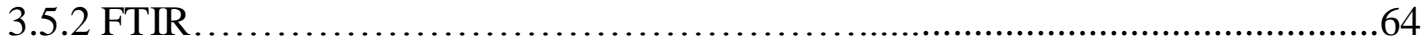

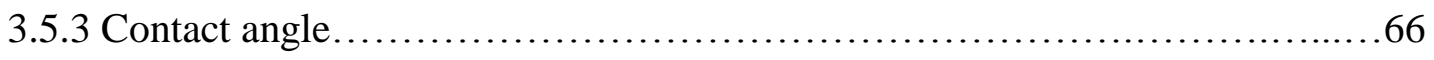

3.5.4 SEM............................................................66

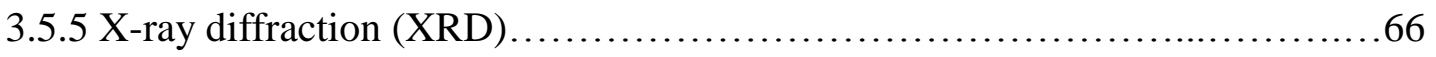

3.5.6 UV-Vis spectrometry..........................................66

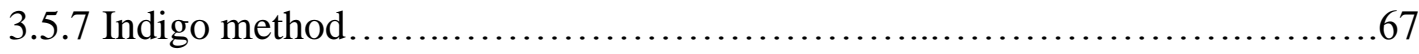

\section{Chapter 4}

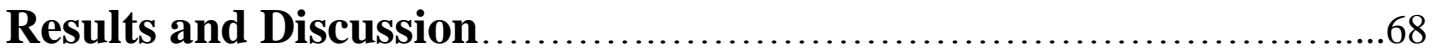


4.1 Polypropylene membrane ozonation.....................................68

4.1.1 PPMM ozonated in the aqueous and gaseous phases.....................69

4.1.2 Washing test.............................................................. 70

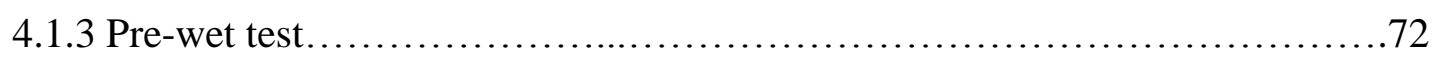

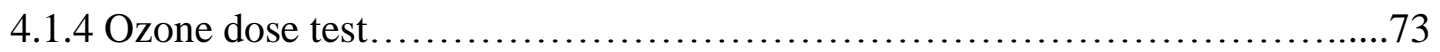

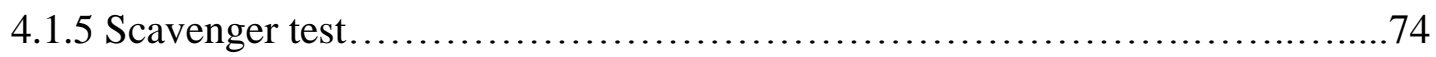

4.1.6 pH test............................................................. 75

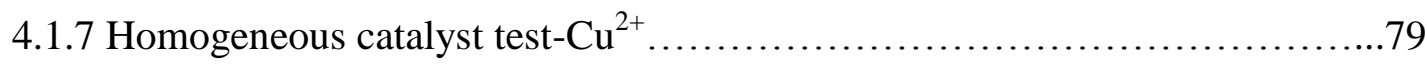

4.1.8 Investigative tests of the mechanism in homogeneous catalyst $\mathrm{Cu}^{2+} \ldots \ldots \ldots . .80$

4.1.8.1 Application of scavengers to the $\mathrm{Cu}^{2+}$ catalytic ozonation................80

4.1.8.2 The effect of anion $\mathrm{SO}_{4}{ }^{2-}$ on the $\mathrm{Cu}^{2+}$ catalytic ozonation..................82

4.1.8.3 $\mathrm{Cu}^{2+}$ catalytic ozonation at a low temperature $\left(0^{\circ} \mathrm{C}\right) \ldots \ldots \ldots \ldots . . . \ldots . . . . .84$

4.1.8.4 Investigation of ozone solubility in distilled water undergoing $\mathrm{Cu}^{2+}$

catalytic ozonation........................................... 86

4.1.8.5 Hydroxyl radical capture by Nitrobenzene (NB) applied to ozonation with

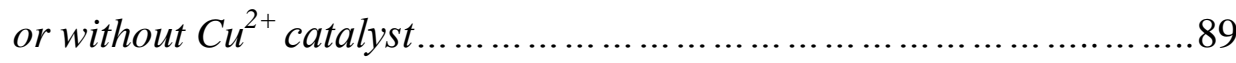

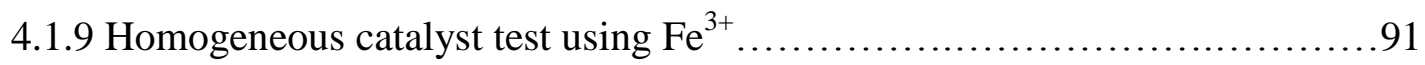

4.2 Polypropylene film ozonation........................................ 92

4.2.1 BOPP ozonated in the aqueous and gaseous phases.......................92

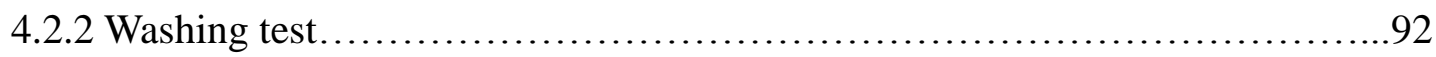

4.2.3 Peroxide aging test of BOPP .......................................... 95

4.2 .4 Scavenger test.................................................... 96

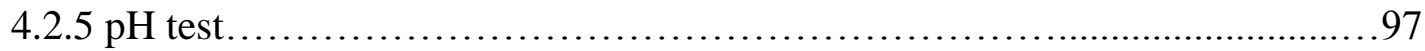

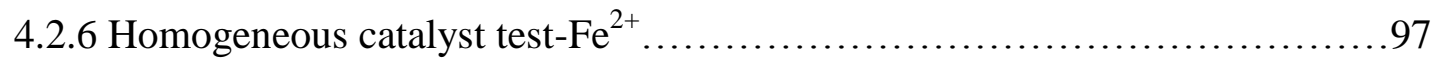

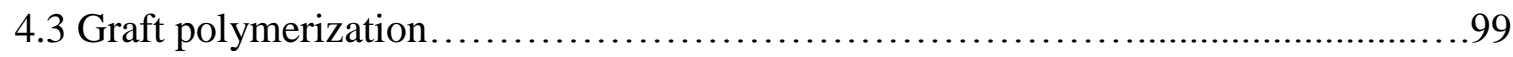

4.3.1 Graft polymerization and FTIR analysis..............................99

4.3.1.1AAm grafted to PPMM and FTIR analysis.........................99

4.3.1.2 Polyethylene glycol (PEG) grafted to PPMM and FTIR analysis.......102

4.3.1.3 AAm grafted to BOPP and FTIR analysis............................105

4.3.2 Graft degree...................................................... 105 
4.3.2.1 Graft degree of PРMM..........................................105

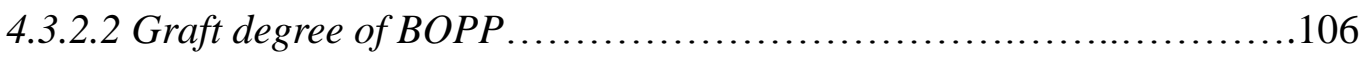

4.3.3 Contact angle test............................................... 106

4.3.3.1 Contact angle of the AAm grafted PPMM...........................106

4.3.3.2 Contact angle of HEMA grafted PPMM............................107

4.3.3.3 Contact angle of PEG grafted PPMM.............................107

4.3.3.4 Contact angle of AAm grafted BOPP..............................108

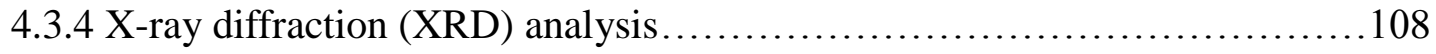

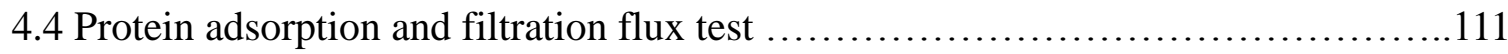

4.4.1 AAm grafted PPMM.............................................. 112

4.4.2 PEG grafted PPMM.............................................. 114

4.4.3 HEMA grafted PPMM............................................ 115

4.4.4 Catalytic grafted PPMM......................................... 116

4.4.5 Formula of filtration flux and fouling ................................116

4.5 SEM images........................................................... 117

4.5.1 SEM images of AAm grafted PPMM.................................117

4.5.2 SEM images of BSA filtration test for PPMM grafted by AAm...........118

4.5.3 SEM images of BOPP after AAm graft polymerization....................119

4.5.4 SEM images of the BSA adsorption test on BOPP ......................120

4.6 Discussion of the Mechanism and Reaction Models.......................122

4.6.1 Kinetics of ozonation of membrane and film surfaces.....................122

4.6.2 Scavengers and the mechanism....................................... 129

4.6.3 The catalytic pathways.............................................. 132

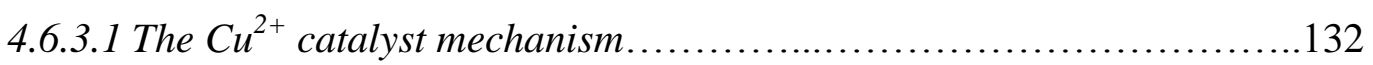

4.6.3.2 Fe(III) catalyst...............................................135

\section{Chapter 5}

Conclusions 


\section{Appendixes}

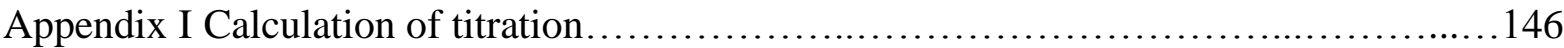

Appendix II Standard Sodium thiosulfate solution preparation...............................147

Appendix III Calibration of Sodium Thiosulfate solution..................................148

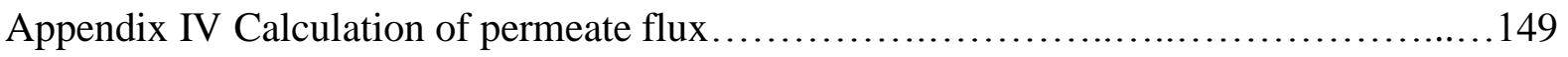

Appendix V Test and Calculation of Ozone Concentration.................................150

Appendix VI A summary of the principle infrared bands and their assignments...............151

Appendix VII Experimental Data Tables of PPMM reaction with ozone......................152

Appendix VIII Experimental Data Tables of scavenger test of PPMM reaction with ozone.

Appendix IV Experimental Data Tables of washing test of PPMM reaction with ozone......155

Appendix X Experimental Data Tables of Pre-wet test of PPMM reaction with ozone......157

Appendix XI Experimental Data Tables of different pH test of PPMM reaction with ozone.

Appendix XII Experimental Data Tables of catalyst test of PPMM reaction with ozone....162 Appendix XIII Experimental Data Tables of BOPP reaction with ozone....................173

Appendix XIV Experimental Data Tables of peroxide concentration aging of BOPP........175

Appendix XV Experimental Data Tables of BOPP aqueous ozonation with scavengers.....176

Appendix XVI Experimental Data Tables of washing test of BOPP reaction with ozone.....178 Appendix XVII Experimental Data Tables of different pH test of BOPP reaction with ozone

Appendix XVIII Experimental Data Tables of catalyst test of BOPP reaction with ozone...180

References. 


\section{LIST OF TABLES}

Tables

Page Numbers

Table 2.1 Membrane classification................................................4

Table 2.2 Ozone properties...................................................... 11

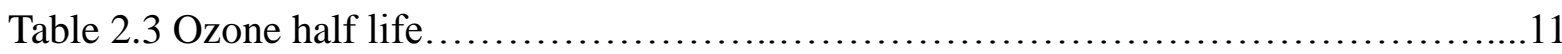

Table 2.4 Solubility of ozone in different concentration and at different temperature.........12

Table 2.5 A comparison of reported reaction orders for the decay rate of ozone in phosphate buffered solutions of demineralized water .................................. 15

Table 2.6 List of other membrane anti-fouling modification..........................48

Table 3.1 Description of the equipment used in the graft polymerization reactor system .....52

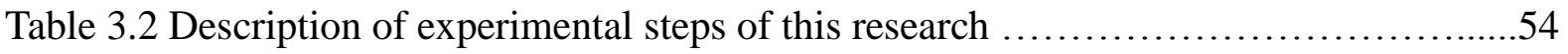

Table 4.1 Dissolved ozone concentration testing in pure distilled water and in catalytic

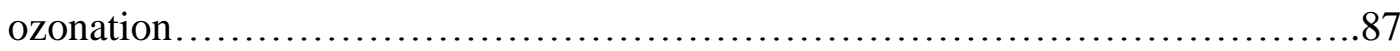

Table 4.2 Dissolved ozone concentration of $\mathrm{Cu}^{2+}$ catalytic ozonation at different temperatures

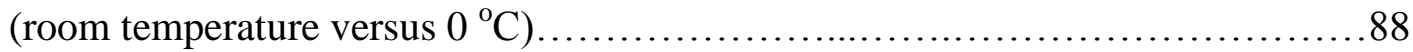

Table 4.3 Graft degree of the PPMM with monomers AAm and PEG....................105

Table 4.4 Graft degree of BOPP with the monomer AAm.............................106

Table 4.5 Contact angle of the PP membrane modified by ozonation and AAm graft.......107

Table 4.6 Contact angle of the PPMM modified by ozonation and HEMA graft.............107

Table 4.7 Contact angle of the PEG graft on PPMM.................................. 108

Table 4.8 Contact angle of the BOPP film modified by ozonation and AAm graft...........108 


\section{LIST OF FIGURES}

Figures

Page Numbers

Fig 2.1 Schematic of membrane fouling.... 5

Fig 2.2Typical concentration profiles of ozone against time obtained in ozone absorption in water at different temperature

Fig 2.3 Variation of the apparent pseudo first-order rate conatant of ozone decomposition in buffered distilled water with $\mathrm{pH}$. ...

Fig 2.4 Variation of the concentration of ozone with the depth of liquid penetration during its absorption at steady state.

Fig 2.5 Reaction time evolution of the ozone decomposition reaction with $\mathrm{pH}$ at $20{ }^{\circ} \mathrm{C} \ldots \ldots .19$

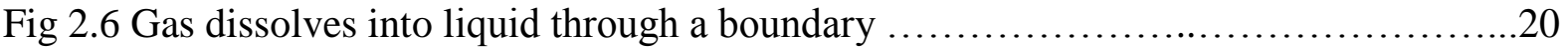

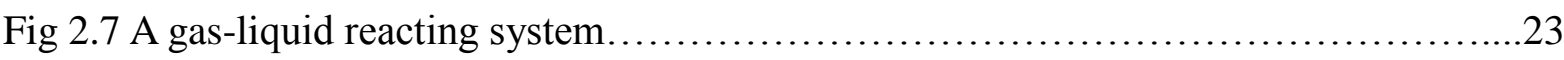

Fig 2.8 Typical ozone concentration profiles....................................... 26

Fig 2.9 Standard potentials of ozone in acidic solution.............................26

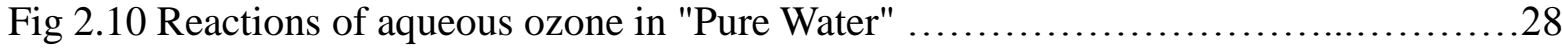

Fig 2.11 Reactions of aqueous ozone in the presence of materials $\mathrm{M}$ which react with $\mathrm{O} \cdot$, or Which interact with $\cdot \mathrm{OH}$ radicals by scavenging and/or converting $\cdot \mathrm{OH}$ into $\mathrm{HO}_{2} \cdot$.

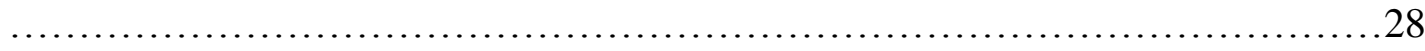

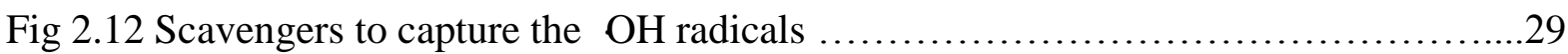

Fig 2.13 Scheme of reactions of aqueous ozone. ..................................... 30

Fig 2.14 Successive half-lives of ozone vs. concentration of carbonate for different $\mathrm{pH}$ values

Fig 2.15 Relative concentration of ozone vs. time for different initial $\mathrm{O}_{3}$ concentrations at high scavenger concentration................................................

Fig 2.16 Radical graft polymerization........................................... 38

Fig 2.17 Schematic illustration of ozone-induced graft polymerization ....................40

Fig 2.18 Fluxes for virgin and modified membranes during cross-flow MF of BSA solution

Fig 2.19 flux recovery efficiencies with water flushing at the end of operation .42 
Fig 2.20 Permeate volume vs. filtration time for cross-flow filtration .43

Fig 2.21 Intrinsic viscosity of polypropylene flake at various levels of ozone treatment.....45

Fig 2.22 Intrinsic viscosity of polypropylene flake at various levels of ozone treatment......45

Fig 2.23 Carbonyl contents of ozonized stabilized and unstabilized polypropylene flake FL-B and FL-A respectively..............................................46

Fig 2.24 Carbonyl contents of ozonized polypropylene flake. Similar data for FL-C, a polypropylene flake provide a reasonable comparison. .46

Fig 2.25 Peroxide generation of BOPP with 3.7wt\% ozone aqueous phase and gaseous phase

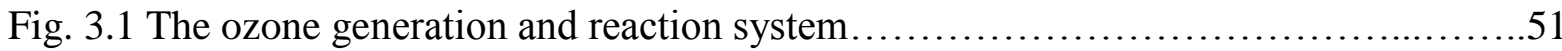

Fig 3.2 Schematic illustration of the ozone generation and reactor system $\ldots \ldots \ldots \ldots \ldots \ldots . \ldots 1$

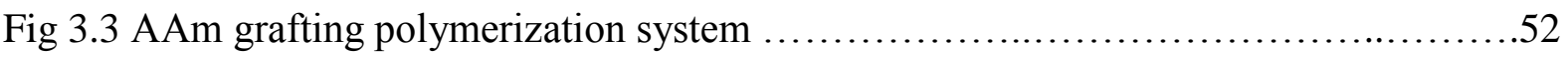

Fig 3.4 Schematic illustration of membrane flux test system........................ 57

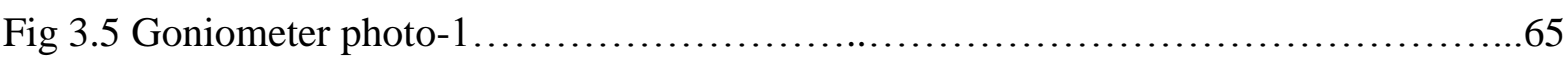

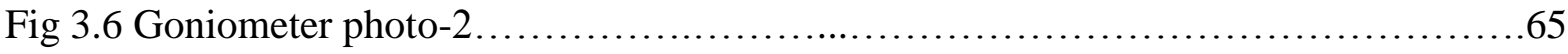

Fig 4.1 Peroxide concentration after ozonation of PPMM in the aqueous and gaseous phases

Fig 4.2 Comparison of the peroxide concentrations of PPMM ozonated in gaseous phase with

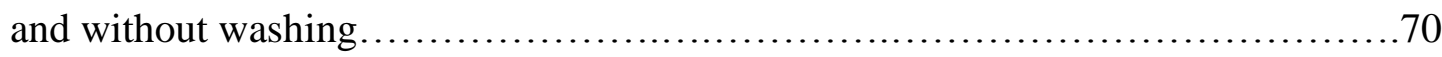

Fig 4.3 Comparison of peroxide concentration of PPMM ozonated in aqueous solution with

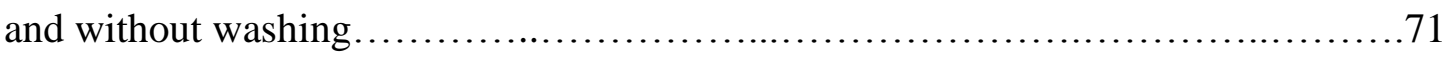

Fig 4.4 Reaction of ozone with PPMM to create hydroperoxide $\ldots \ldots \ldots \ldots \ldots \ldots \ldots \ldots \ldots \ldots . .72$

Fig 4.5 Ozone reacted with PPMM to create small molecules by chain scission....................72

Fig 4.6 Results of aqueous phase ozonation of pre-wetted PPMM.......................73

Fig 4.7 Different ozonation doses - $1.0 \mathrm{wt} \%$, and $3.0 \mathrm{wt} \%$ - applied in the aqueous phase.. 74

Fig 4.8 Peroxide generation of PPMM ozonated in distilled water with sodium carbonate as

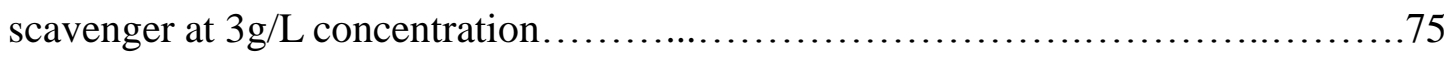

Fig 4.9 Peroxide generation at different $\mathrm{pH}$ levels with $3.0 \mathrm{wt} \%$ of applied ozone dose and 30 min ozonation 77 
Fig 4.10 Peroxide generation with different ozone doses applied at different $\mathrm{pH}$ levels......77

Fig 4.11 Peroxide generation of ozonation for 30 minutes and at $3.0 \mathrm{wt} \%$ using $\mathrm{Cu}^{2+}$ catalyst in DW .78

Fig 4.12 Peroxide generation at different ozonation time using $\mathrm{Cu}^{2+}$ catalyst in DW and in the gaseous phase. .78

Fig 4.13 Scavenger's effect on the aqueous phase and $\mathrm{Cu}^{2+}$ catalytic ozonation- $3.0 \mathrm{~g} / \mathrm{L}$

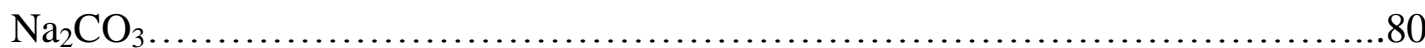

Fig 4.14 The effect of scavengers on $\mathrm{Cu}^{2+}$ catalytic ozonation at room temperature..........81

Fig 4.15 The effect of scavengers on $\mathrm{Cu}^{2+}$ catalyzed ozonation at $0^{\circ} \mathrm{C} \ldots \ldots \ldots \ldots \ldots \ldots . . . . . .2$

Fig 4.16 Effect of $\mathrm{SO}_{4}{ }^{2-}$ on peroxide generation in non-catalyzed ozonation of PPMM.........83

Fig 4.17 Effect of $\mathrm{SO}_{4}{ }^{2-}$ on peroxide generation in catalyzed ozonation of PPMM ..........84

Fig 4.18 Different concentrations of $\mathrm{Cu}^{2+}$ catalyst applied to $0^{\circ} \mathrm{C}$ ozonation at $15 \& 30 \mathrm{~min} .85$

Fig 4.19 Different concentrations of $\mathrm{Cu}^{2+}$ catalyst applied to $0^{\circ} \mathrm{C}$ and $22^{\circ} \mathrm{C}$ ozonation.......86

Fig 4.20 UV scan chart for $25 \mathrm{~min}$ of aqueous ozonation at $0^{\circ} \mathrm{C}$ with/without catalyst...... 88

Fig 4.21 UV scan chart for $35 \mathrm{~min}$ of aqueous ozonation at $0^{\circ} \mathrm{C}$ with/without catalyst ...... 89

Fig 4.22 Detection of NB captured $\cdot \mathrm{OH}$ in the $\mathrm{Cu}^{2+}$ catalyzed ozonation ....................90

Fig 4.23 Detection of NB captured $\cdot \mathrm{OH}$ in non-catalyzed ozonation $\ldots \ldots \ldots \ldots \ldots \ldots \ldots \ldots . \ldots 91$

Fig 4.24 Catalytic ozonation of PPMM using $\mathrm{Fe}^{3+}$ catalyst ..............................92

Fig 4.25 Peroxide generation in the ozonation of BOPP in the aqueous and gaseous phases.94 Fig 4.26 Comparison of peroxide concentration on BOPP treated by gaseous phase ozonation

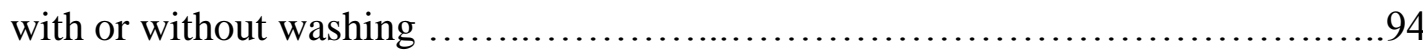

Fig 4.27 Comparison of peroxide concentration on BOPP treated by aqueous phase ozonation

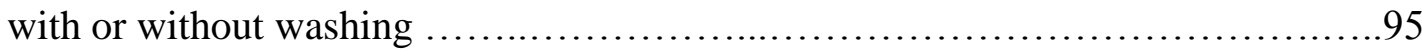

Fig 4.28 Results of the aging test of peroxides generated from aqueous ozonation of BOPP.96

Fig 4.29 Peroxide generation from aqueous ozonation of BOPP with and without $3.0 \mathrm{~g} / \mathrm{L}$ of sodium carbonate as a scavenger....................................... 97

Fig 4.30 Peroxide generation of BOPP at different $\mathrm{pH}$ levels ..........................98

Fig 4.31 Effect of different ozonation times for $\mathrm{Fe}^{3+}$ catalyzed ozonation of BOPP...........99

Fig 4.32 Results of FTIR analysis of PPMM after aqueous phase ozonation and AAm grafting 
Fig 4.33 Results of FTIR analysis of PPMM after gaseous phase ozonation and AAm grafting 101

Fig 4.34 Comparison of results of FTIR analysis of PPMM after aqueous and gaseous phase ozonation and AAm grafting .101

Fig 4.35 Chemical reaction schematic of AAm graft onto PPMM. 102

Fig 4.36 Comparison of FTIR results for aqueous phase ozonated PPMM grafteded with PEG of different molecular weight

Fig 4.37 FTIR of BOPP with 3.0 wt\% applied ozone dose for A15, A45 + AAm graft polymerization. 104

Fig 4.38 FTIR of BOPP with $3.0 \mathrm{wt} \%$ applied ozone dose for A45 and G45 + AAm graft polymerization. 104

Fig 4.39 XRD of PPMM with 6 and 15 min of aqueous ozonation. 110

Fig 4.40 XRD of PPMM ozonated for $15 \mathrm{~min}$ in aqueous phase with and without gratfing of AAm .110

Fig 4.41 XRD of PPMM ozonated for $15 \mathrm{~min}$ in gaseous phase with and without gratfing of AAm 111

Fig 4.42 Dead-end BSA filtarion of virgin, A18, and G18 + AAm modified PPMM. 113

Fig 4.43 Second filtration test (after back flush) of AAm modified PPMM .113

Fig 4.44 BSA filtration test of PEG modified PPMM. 114

Fig 4.45 BSA filtration test of HEMA modified PPMM. 115

Fig 4.46 BSA filtration test of $\mathrm{Fe}^{3+}$ and $\mathrm{Cu}^{2+}+\mathrm{AAm}$ modified PPMM. 115

Fig 4.47 SEM images of PPMM grafted by AAm................................118

Fig 4.48 SEM images of BSA fouled PPMM 119

Fig 4.49 SEM images of BOPP grafted with AAm. 120

Fig 4.50 SEM images of BOPP adsorbed with BSA after grafted with AAm 121

Fig 4.51 SEM images of PPMM adsorbed with BSA after grafted with AAm and PEG ....122

Fig 4.52 Schematic of ozone reacting with PPMM in the aqueous phase .123

Fig 4.53 The two types of reactions of ozone in aqueous solution 131

Fig 4.54 Peroxide generation accelerated by hydroxyl radicals 133

Fig 4.55 Peroxide generation accelerated by oxygen radicals .135 


\section{Chapter 1}

\section{Introduction}

Many membranes and plastic films are polymers. They are widely used in filtration, or in biomedical fields, for drug release, blood and protein packaging materials, artificial organs, medical devices [Xu et al., 2003]. They are chemically nonreactive, easy to process, and have good mechanical and thermal properties; however, their surfaces are usually hydrophobic, and non-bondable or non-compatible. Without treatment, the hydrophobic surface cause anti-coagulation [Dasgupta, 1990] and filtration fouling, which limit their applications in many areas.

Membrane filtration is a separation process and has been used extensively in various applications such as wastewater treatment, chemical industry, pharmaceutical industry, etc. However, the filtration flux decline caused by membrane fouling constitutes a major obstacle to the wide-scale applications (Escobar et al., 2005). Research results indicated that increasing membrane surface hydrophilicity could effectively inhibit membrane fouling (Belfer et al., 1998; Boutevin et al., 2002; Chang et al., 2008; Escobar et al., 2005; Gullinkala et al., 2008). To extend the lifetime of the membranes, modifying surfaces of the membranes to increase their hydrophilicity is thus very important. In this research, polypropylene microfiltration membrane (PPMM), a widely used commercial membrane, was selected for surface modification by ozonation and graft polymerization.

On the other hand, biaxial oriented polypropylene (BOPP) film is cheap, chemically nonreactive, mechanically strong and heat resistant. However, their surfaces are usually hydrophobic and weak-bondable, which limit their applications, such as in printing and lamination of packaging, and in biomedical applications [Dasgupta, S., 1990]. Hydrophobic surfaces cause anticoagulation, adhesion and coating when they come into contact with biomedical materials (Murakami et al., 2003). BOPP film is usually pretreated before used in further processing. Polypropylene films required some type of surface modification prior to commercial uses. In packaging applications, corona or flame treatments are widely used to improve the bondability. In biomedical applications, grafting polymerization is used to improve the hydrophilicity. 
Various techniques can be used to increase the polymeric surface hydrophilicity. There are plasma treatment, irradiation with gamma-rays, corona discharge, ion beam treatment, and UV radiation (Hua et al., 2008), etc. Compared to those techniques, ozonation is inexpensive and easy to carry out. Moreover, ozonation modifies surfaces uniformly even with complicated shapes (Yuan et al., 2002). It can be conducted in either gaseous phase or aqueous phase. In fact, aqueous ozonation facilitates the rapid decomposition of molecular ozone to form free radicals, which are stronger oxidants than ozone itself. In addition, aqueous ozonation enables the easy addition of catalysts and additives to enhance the ozonation efficiency.

When polymers are exposed to ozone, active peroxide groups can be introduced onto the surface. These active peroxide groups are capable of initiating graft polymerization of vinyl monomers with hydrophilic groups. The hydrophilicity of the polymer surfaces thus can be improved (Gu et al., 2009).

In my MSc thesis (Gu, 2008), the ozonation of BOPP was reported. Further study of grafting polymerization and protein adsorption were to explore in this research. Hydrophlicity of surfaces depends on the amount of hydrophilic monomers grafted on the surfaces, which in turn depends on the amount of peroxides generated.

Information of polymeric membranes modified by ozonation in the gaseous phase and liquid phase was found in literature; reports of ozonation in the presence of catalysts to modify polyethylene films were found recently, which were published by our research group members. However the catalytic ozonation of polypropylene membrane and film for their surface modification, and the mechanism of homogeneous catalytic ozonation of polymer surfaces has not been reported.

Therefore, the objectives of this study were to enhance surface hydrophilicity of polypropylene membranes and films by homogeneous catalytic ozonation, and to explore the mechanisms of the conducted ozonation.

In Chapter 2, the literature survey is conducted. In Chapter 3, experimental methodology is described, including materials, experiment setup, procedure, and analytical methods. Chapter 4 is the results and discussion regarding dosage study of ozonation applied to PP membranes and films; washing tests; scavenger tests; $\mathrm{pH}$ tests; homogeneous catalyst 
tests of $\mathrm{Cu}^{2+}, \mathrm{Fe}^{3+}$, and the mechanism study of $\mathrm{Cu}^{2+}$ catalyst; grafting tests; flux tests of filtration using modified PPMM, and protein absorption tests for modified BOPP. In Chapter 5 , the conclusions of this study are presented. 


\section{Chapter 2}

\section{Literature Study}

\subsection{Membranes, films and their modifications}

In this section, membranes, films, and their applications and modifications are discussed, the fouling of membranes are also introduced.

\subsubsection{Membrane classification}

Membranes are used extensively in water/wastewater treatment, separations in the food, dairy, paper, textile, chemical purification, biotechnology industries, medical field, and drug release and delivery. The modern era of membrane technologies for water purification was launched in the late 1950s with the development of asymmetric cellulose acetate (CA) membranes for reverse osmosis (RO). The materials that followed CA and its derivatives (i.e., cellulose diacetate and cellulose triacetate) are polyamide (PA), polysulfone, polyvinylidene fluoride (PVDF), and polyolefin. Thin film composite (TFC) membranes may be made from a variety of polymers consisting of several different materials for the substrate, the thin film, and other functional layers. Polymeric membrane materials now dominate the commercial product market (Escobar et al., 2005).

Membranes are classified according different pore size and applications, as shown in Table 2.1.

Table 2.1 Membrane classification

\begin{tabular}{|c|c|c|c|}
\hline & Pore Size $(\mathrm{nm})$ & Pressure $($ Bar $)$ & Flux $\left(\mathrm{L} / \mathrm{m}^{2} \cdot \mathrm{h} \cdot \mathrm{bar}\right)$ \\
\hline Microfiltration & $\mathrm{D}>100$ & $0.1-2.0$ & $>50$ \\
\hline Ultrafiltration & $1<\mathrm{D}<100$ & $1.0-3.0$ & $10-50$ \\
\hline Nanofiltration & $0.8<\mathrm{D}<8$ & $5.0-20$ & $1.4-12$ \\
\hline Reverse Osmosis & $\mathrm{D}<1$ & $10-100$ & $0.05-1.4$ \\
\hline
\end{tabular}

D-average diameter of the pores (http://www.lenntech.com/membrane-fouling.htm, Oct, 2008) 
In water purification, microfiltration (MF) membranes remove particulates and control turbidity; ultrafiltration (UF) membranes can provide protection from pathogenic microorganisms (including protozoans, bacteria, and viruses) and partially remove natural dissolved organic matter (NOM); nanofiltration (NF) and reverse osmosis (RO) membranes remove divalent cations (water softening) and can effectively remove natural organic matter (NOM) (Escobar et al., 2005).

\subsubsection{Filtration resistance}

Fouling resistance includes: $\quad R_{m}=$ membrane resistance

$$
\begin{aligned}
& R_{a}=\text { adsorption, biofouling } \\
& R_{p b}=\text { pore plugging } \\
& R_{\mathrm{c}}=\text { cake layer }
\end{aligned}
$$

As shown in Figure 2.1, $R_{a}$ and $R_{p b}$ are irreversible foulings.

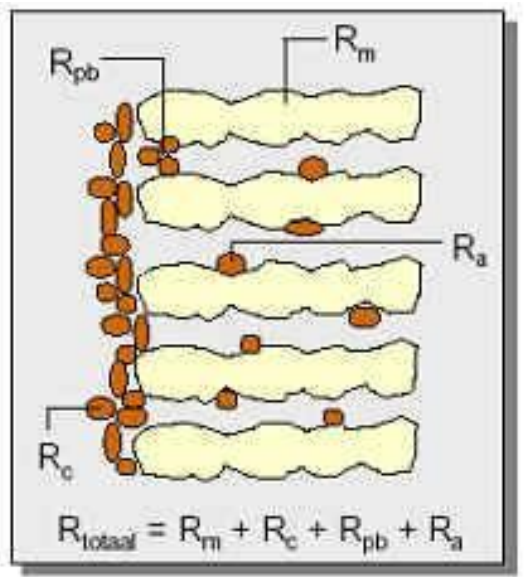

Fig 2.1 Schematic of membrane fouling (http://www.lenntech.com/membrane-fouling.htm, Oct, 2008).

\subsubsection{Membrane Fouling}

The flux decline caused by concentration polarization, and membrane fouling by natural organic matter, proteins and other biomolecules or organic pieces adsorption in the feed stream, is a large challenge. It constitutes a major obstacle to the wide-scale application 
in these industries. While inorganic scaling of membranes by calcium salts can be controlled by adjusting the $\mathrm{pH}$ and adding anti-scalants, natural organic matter is ubiquitous in all water supplies and has been implicated as a major contributor to fouling during filtration of natural water. Concentration polarization and membrane fouling not only decrease membrane permeability, but also shorten membrane life due to the aggressive chemicals necessary for cleaning. When cleaning becomes ineffective, the membranes must be replaced. Concentration polarization decreases the driving force of water flow across the membrane due to a local increase in foulant concentration. The effect is completely reversible, and can be reduced by modifying the flow over the membrane. Membrane fouling itself can occur in two ways: cake formation and adsorption of foulants. Cake fouling is generally reversible by water flushing or backwashing. However, fouling due to the adsorption of foulants is essentially irreversible and can only be treated to a certain extent by aggressive chemical cleaning. Foulant adsorption can occur both on the membrane surface and in the pores. Since hydrophobic adsorption of foulants on membrane surfaces plays a key role in membrane fouling, hydrophilic modification of polymeric membrane surface can be one of the fouling mitigation methods. Many investigations have demonstrated that increasing membrane surface hydrophilicity could effectively inhibit membrane fouling (Hua et al., 2008).

Biotic biofouling caused by an active biofilm and abiotic fouling associated with NOM of a microbial origin. The role of microbial processes in the production of foulants is also important. Biofouling is inherently more complicated than other membrane fouling phenomena. Microorganisms can grow, multiply, and relocate.

In general, biofouling of membranes will lead to a change in biomolecular structure selectively decreasing the permeate flux with time, especially in the filtration of protein, platelet, or cell-containing solutions. It is believed that the increase of hydrophilic moieties on a hydrophobic material surface can effectively reduce its membrane fouling as a consequence of hydrophobic interactions between the biomolecules and the hydrophobic surface (Chang et al., 2008). Therefore, an ideal anti-fouling membrane should possess the excellent mechanical bulk properties, and the anti-fouling characteristics of a hydrophilic surface on the membrane surface and pores. 


\subsubsection{Modifications to Improve Membrane Anti-fouling}

Various methods including blending, coating, adsorbing, chemical-grafting can be used to modify the surface condition. Plasma and radiation-induced grafting have been developed to modify membrane surfaces using hydrophilic modifiers. Ozone appears as a relatively cheap reagent. Ozone is a very active oxidant, and can be easily generated by using a high voltage electric device and using air or pure oxygen as raw materials. Ozonation is widely investigated in polymeric film surface treatment and modification, membrane fouling improvement (Yuan et al., 2002).

Although there are not as many articles as other methods reported, such as ultraviolent (UV) and plasma methods utilized in membrane anti-fouling modification, ozonation and induced graft polymerization have their advantages to improve the surface properties of polymer membranes. Ozonation and the subsequent graft polymerization occur not only on the surface comparing to plasma or UV treatment to the membrane, but also occur to the pore sites. This will help to improve the pore anti-fouling. The content of graft polymerization which is strictly controlled can change the pore size to turn the macro-filtration to micro-filtration.

\subsubsection{Polymeric films and modifications}

Polymer films include polyethylene (PE), polypropylene (PP), polyester (PET), etc. In the last few decades synthetic polymers have replaced several materials, such as metals, and have extended their application to many innovative processes. This is due to their superior physical and chemical characteristics such as high strength to weight ratio, corrosion resistance and chemical inert nature. They are also relatively inexpensive and easy to process. At the same time some of these properties impose a limitation on applications in several new and high technology areas. Thus it is required that their surface properties be modified to suit a particular application without affecting their bulk properties (Sharma et al., 2002).

Surface modification is quite often used to create surfaces with properties considerably different from that of the bulk of the polymeric materials. Since the polymer surfaces are non-reactive, and surface modification involves chemical alteration of the surface layer, it requires the generation of high-energy species such as radicals, ions, and 
molecules to promote a surface reaction. This is achieved by techniques such as flame, plasma, UV, laser, X- ray and $\gamma$-ray, electron beam, ion beam, and corona treatment (Sharma et al., 2002). Ozonation and induced graft polymerization also applies to improve the surface properties of polymeric films.

\subsection{Ozonation and Surface Modification}

In this section, the peroxide generation by ozonation, peroxide decomposition to radicals and the grafting polymerization induced by the radicals are discussed.

\subsubsection{Ozone reactions with polymers}

The reactions of ozone onto polymers lead to numerous chemical modification. Ozone leads rapidly to formation of oxygenated functions and a chemical reaction occurred at the surface of the polymer as well as in the depth of a material. The main functions appearing during treatment are unsaturated compounds, such as ketones, acids, esters, hydroxyl groups, peroxides and hydroperoxides (Robin, 2004).

Surface ozonation is applied in polymer research areas because it has the advantage of uniformly introducing peroxides on the polymer surface even with complicated shapes and offers an easy to handle and relatively inexpensive technique. When polymers exposed to ozone, peroxides are mainly formed in addition to the carbonyl and carboxyl groups. The carbonyl and carboxyl groups themselves are hydrophilic; furthermore, the generated peroxides are capable of initiating polymerization of vinyl monomers, and coupling or immobilizing amide and amino groups, resulting in polymer graft and immobilization onto the ozonated polymeric materials. The reactivity of the peroxidized materials depends on the following factors: peroxide thermal decomposition kinetics, the nature of the generated radicals, the accessibility of peroxides sites, different monomers and monomers diffusion; the degree of polymerization and the length of the chain (Robin, 2004).

This complicated scheme shows that the species R·, HO·, and HOO- are responsible 
for the further abstraction of hydrogen from a neighboring chain of polyolefin, but also and mainly to the $\beta$-scissions, leading to alcohol functions. Moreover, the radicals obtained can react with oxygen giving hydroperoxides. On the other hand, intramolecular rearrangement produces different species leading to esters and ketones (Kulik et al., 1995).

\subsubsection{Peroxide generation and decomposition on polymer surface}

Three typical methods are involved in peroxide generation (ozonization, UV irradiation, and plasma treatment). The generated peroxides can decompose to radicals thus induce the graft polymerization. The polymer peroxides formed are decomposed either thermally or using a reducing agent in aqueous solution. Both decomposition methods are commonly used for the initiation of graft polymerization of water-soluble monomers. An important factor governing the initiation of graft polymerization is the accessibility of monomer solution to the polymer peroxides, which must be distributed on the outermost surface and into the bulk phase of the treated polymer substrate (Kulik et al., 1995). To examine the peroxide accessibility, the peroxide density is determined by two methods: iodide and peroxidase methods (Dasgupta, 1990).

Plasma treatment seems to generate most of the peroxides in the surface layer of film, while UV irradiation and ozonation will generate peroxides in the subsurface region of film. Peroxide generation depended on both the nature of polymer substrate and the method of polymer oxidation

\subsubsection{Redox Decomposition of Peroxide}

The peroxide can decompose with Mohr's salt $\left(\mathrm{FeSO}_{4} \cdot\left(\mathrm{NH}_{4}\right)_{2} \mathrm{SO}_{4} \cdot 6 \mathrm{H}_{2} \mathrm{O}\right)$ at concentrations ranging of $10^{-6} \mathrm{M}, \mathrm{Fe}^{+2}$ reacts with $\mathrm{ROOH}$ (peroxide) to become $\mathrm{Fe}^{+3}$ and $\mathrm{ROOH}$ break to $\mathrm{RO}$ and $\cdot \mathrm{OH}$ radicals. Under these experimental conditions ferrous ions at a concentration less than $10^{-6} \mathrm{M}$ did not cause any measurable decrease in the peroxide density. A small reduction of peroxide density was observed when the Mohr's salt concentration was in the range from $10^{-6}-10^{-5} \mathrm{M}$ (Kulik et al., 1995). 


\subsubsection{Thermal Decomposition of Peroxide}

The peroxide can also break into radicals by thermal decomposition. Heating the polymer samples led to more prominent decomposition of peroxides than the redox reaction with ferrous ions at $25^{\circ} \mathrm{C}$, as is obvious from the comparison.

The extent of thermal decomposition of peroxide at $65^{\circ} \mathrm{C}$ depended on both the nature of polymer substrate and the method of peroxide detection. Raising the decomposition temperature to $85^{\circ} \mathrm{C}$ led to enhancement of peroxide decomposition.

Since the thermal decomposition of peroxide did not obey a simple first-order reaction, it may be assumed that several types of peroxides are formed on and in the polymer substrates (Kulik et al., 1995).

$$
\begin{aligned}
& d \mathrm{Per}_{m} / d t=-k_{m} \mathrm{Per}_{m} \\
& d \mathrm{Per}_{l} / d t=-k_{l} \mathrm{Per}_{l} \\
& \mathrm{Per}=\mathrm{Per}_{m}+\mathrm{Per}_{l}
\end{aligned}
$$

After integrate Equations (2.1) and (2.2), we obtain

$$
\text { Per } / \mathrm{Per}^{o}=\mathrm{Per}_{m}^{o} / \mathrm{Per}^{o} \exp \left(-k_{m} t\right)+\mathrm{Per}_{l}^{o} / \mathrm{Per}^{o} \exp \left(-k_{l} t\right)
$$

For $t>>1 / k_{m}$

$$
\log \left(\mathrm{Per}^{\mathrm{Per}}{ }^{\circ}\right)=\log \left(\mathrm{Per}_{l}^{o} / \mathrm{Per}^{\circ}\right)-0.434 k_{l} t
$$

Where $\mathrm{Per}$ is the total peroxide density at time $\mathrm{t} ; \mathrm{Per}^{o}$ is the total initial peroxide density; $\mathrm{Per}_{m}^{o}$ is the initial density of the most decomposable peroxide with the decomposition rate constant of $k_{m} . \mathrm{Per}^{\circ} / \mathrm{Per}^{o}$ and $k_{l}$ calculated according to experimental results. $k_{l}$ is practically independent of polymer substrate, peroxide generation method, and peroxide assay, while $\mathrm{Per}^{\circ} / \mathrm{Per}^{\circ}$ seems to be dependent on all these factors (Kulik et al., 1995).

\subsubsection{Graft polymerization}

The surfaces of ozone-pretreated polymeric membranes or films were subjected to 
further modification. The grafting of monomers with a vinyl function groups to ozonated polyolefin and other materials can increase surface hydrophilicity. There are several routes one may follow to achieve this goal, and the grafting steps may be carried out under various ambient conditions. The monomers may be used in the vapor phase, liquid phase, in pure or solution, possibly with dry substrates, and the medium may be either organic, aqueous, or a mixture of the two [Dasgupta, 1990]. The grafting reaction was initiated by heat or the addition of $\mathrm{FeCl}_{2} \cdot 2 \mathrm{H}_{2} \mathrm{O}$ to discompose the peroxide into the radicals $\mathrm{O}$. and $\cdot \mathrm{OH}$, which induce the polymerization. Different monomers or oligmers can be used, such as poly ethylene glycol (PEG), acrylic acid (AAc), acrylicamide (AAm), 2-hydroxyethyl methacrylate (HEMA), sodium salt of styrene sulfonic acid (NaSS), $N, N$-dimethylacrylamide (DMAA), N,N- (dimethylamino)ethyl methacrylate (DMAEMA) and 3-dimethyl (methacryloyl ethyl)- ammonium propanesulfonate (DMAPS) (Kulik et al., 1995).

\subsection{Ozone Properties}

In this section, ozone properties, ozone dissolved in water and affected by $\mathrm{pH}$ were discussed.

\subsubsection{Properties of ozone and comparison with oxygen}

The ozone properties and half life of ozone are shown in Tables 2.2 and 2.3. 
Table 2.2 Ozone properties

\begin{tabular}{|c|c|c|}
\hline Property & $\underline{\text { Ozone }}$ & vs. Oxygen \\
\hline Molecular Formula: & $\mathrm{O}_{3}$ & $\mathrm{O}_{2}$ \\
\hline Molecular Weight: & 48 & 32 \\
\hline Color: & light blue & colorless \\
\hline Smell: & $\begin{array}{l}\text { - clothes after being outside on } \\
\text { clothesline } \\
\text { - photocopy machines } \\
\text { - smell after lightning storms }\end{array}$ & - odorless \\
\hline $\begin{array}{l}\text { Solubility in Water (0-deg } \\
\text { C): }\end{array}$ & 0.64 & 0.049 \\
\hline Density (g/L): & 2.144 & 1.429 \\
\hline $\begin{array}{c}\text { Electrochemical Potential, } \\
\text { V: }\end{array}$ & 2.07 & 1.23 \\
\hline
\end{tabular}

(http://www.ozoneapplications.com/info/ozone_properties.htm, Oct, 2010)

Table 2.3 Ozone half life-typical $\mathrm{O}_{3}$ half-life vs. Temperature

Gaseous

\begin{tabular}{|c|c|}
\hline Temp (C) & half-life * \\
\hline-50 & 3-months \\
\hline-35 & 18-days \\
\hline-25 & 8-days \\
\hline 20 & 3-days \\
\hline 120 & 1.5-hours \\
\hline 250 & 1.5- seconds \\
\hline
\end{tabular}

Dissolved in Water (pH 7)

\begin{tabular}{|c|c|}
\hline $\begin{array}{c}\text { Temp } \\
(\mathrm{C})\end{array}$ & half-life \\
\hline 15 & 30 -minutes \\
\hline 20 & 20 -minutes \\
\hline 25 & 15 -minutes \\
\hline 30 & 12 -minutes \\
\hline 35 & 8 -minutes \\
\hline
\end{tabular}

(http://www.ozoneapplications.com/info/ozone_properties.htm, Oct, 2010)

Table 2.4 shows the solubility of ozone which depends on the water temperature and the ozone concentration in the gas phase: Units in $\mathrm{mg} / \mathrm{L}$ or $\mathrm{ppm}$. 
Table 2.4 Solubility of ozone in different concentration and temperature

\begin{tabular}{|c|c|c|c|c|}
\hline $\mathrm{O}_{3} \mathrm{Gas}$ & $5{ }^{\circ} \mathrm{C}$ & $10{ }^{\circ} \mathrm{C}$ & $15{ }^{\circ} \mathrm{C}$ & $20{ }^{\circ} \mathrm{C}$ \\
\hline $1.5 \%$ & 11.09 & 9.75 & 8.40 & 6.43 \\
\hline $2 \%$ & 14.79 & 13.00 & 11.19 & 8.57 \\
\hline $3 \%$ & 22.18 & 19.50 & 16.79 & 12.86 \\
\hline
\end{tabular}

(http://www.ozoneapplications.com/info/ozone_properties.htm, Oct, 2010)

\subsubsection{Decomposition of ozone in water and $\mathrm{pH}$ effect}

\subsubsection{Effect of hydroxide ions $\mathrm{OH}$ on ozone decomposition}

Ozone is a very strong oxidant $\left(E_{0}=2.07 \mathrm{~V}\right)$, which is second in this regard only to the hydroxyl radical $\left(E_{0}=2.80 \mathrm{~V}\right)$ and substantially exceeds chlorine $\left(E_{0}=1.36 \mathrm{~V}\right)$. Ozone reacts with water to give oxygen. The rate of this reaction noticeably grows as $\mathrm{pH}$ is raised, which is due to the catalyzing effect of hydroxide ions $\mathrm{OH}^{-}$. The mechanism includes a chain of successive reactions yielding $\mathrm{H}_{2} \mathrm{O}_{2}, \mathrm{O}_{3}{ }^{-}, \cdot \mathrm{OH}$ and $\mathrm{HO}_{2} \cdot$ radicals (Beltran, 2004). The radical products initiate chain decomposition of ozone. The disinfecting and oxidizing efficiency of ozone in water depends on its concentration, which is determined by the rate of its decomposition. The kinetics of ozone decomposition in water and the effect of various factors on the rate of this process were investigated by lots of researchers. However, the results obtained are rather contradictory. For example, different orders of the reaction of ozone decomposition in water, including first (Teramoto et al., 1981), first-and-a-half (Sotelo et al., 1987; Ku et al., 1996), and second (Gurol et al., 1982), and markedly different rate constants have been reported.

The decomposition of ozone is catalyzed by $\mathrm{OH}^{-}$ions and occurs by the scheme (Ershov et al., 2008).

$$
\mathrm{O}_{3}+\mathrm{OH}^{-} \rightarrow \mathrm{HO}_{2}^{-}+\mathrm{O}_{2}
$$




$$
\begin{aligned}
& \mathrm{O}_{3}+\mathrm{HO}_{2}^{-} \rightarrow \cdot \mathrm{O}_{2}^{-}+\cdot \mathrm{OH}+\mathrm{O}_{2} \\
& \mathrm{O}_{3}+\cdot \mathrm{O}_{2}^{-} \rightarrow \cdot \mathrm{O}_{3}^{-}+\mathrm{O}_{2} \\
& \cdot \mathrm{O}_{3}^{-}+\mathrm{H}_{2} \mathrm{O} \longrightarrow \cdot \mathrm{OH}+\mathrm{OH}^{-}+\mathrm{O}_{2}
\end{aligned}
$$

The reaction of $\mathrm{O}_{3}$ with $\mathrm{OH}^{-}$leads to the loss of additional two molecules of ozone and regeneration of the $\mathrm{OH}^{-}$ion. The chain process of ozone decomposition occurs as a result of reactions involving $\cdot \mathrm{OH}$ and $\mathrm{HO}_{2} \cdot$ radicals. The chain is terminated by their recombination. A second-order reaction of ozone decomposition in water was observed in the $\mathrm{pH}$ range 6.0-9.5. The study was carried out in the presence of $0.1-1.0 \mathrm{M}$ of a borate or phosphate as a buffering additive, rather than in pure water. The values of the constants, measured at the same $\mathrm{pH}$ values as those in the present study, strongly depended on the additive used and its content in solution and differed by one to two orders of magnitude. All these circumstances indicated that the additives are involved in the ozone decomposition (Ershov et al., 2008).

The decomposition of ozone becomes faster as temperature rises. The second-order reaction of ozone decomposition in water at $\mathrm{pH} 4.7$ depends on temperature in the Arrhenius coordinates. In the temperature range $9-37^{\circ} \mathrm{C}$, this dependence is described by the equation (Ershov et al., 2008).

$$
k(\mathrm{~T})=\mathrm{k}_{19} \exp \{76.0[(\mathrm{~T}-292) / 292 R T]\}
$$

Where $k_{19}$ is the rate constant at a temperature of $19^{\circ} \mathrm{C}$, and $R$ is the universal gas constant $\left(8.314 \mathrm{~J} \mathrm{~mol}^{-1} \mathrm{~K}^{-1}\right)$. The activation energy of ozone decomposition was $76.0 \pm 8.3 \mathrm{~kJ} \mathrm{~mol}^{-1}$ In spectrophotometric analysis of cell reactor, at $20^{\circ} \mathrm{C}, \mathrm{pH} \mathrm{3,7}$ and 9 adjusted with $\mathrm{H}_{2} \mathrm{SO}_{4}$, and $\mathrm{NaOH}$, kinetics of ozone decomposition depends on $\mathrm{pH}$ (Beltran, 2004):

$$
\begin{aligned}
& \mathrm{pH} \text { 3: }-\mathrm{r}_{O_{3}}=\mathrm{k} \mathrm{c}\left(\mathrm{O}_{3}\right)_{\mathrm{a}}{ }^{3 / 2} \\
& \mathrm{pH} 7:-\mathrm{r}_{O_{3}}=\mathrm{k} \mathrm{c}\left(\mathrm{O}_{3}\right)_{\mathrm{a}}{ }^{3 / 2 \sim 1}
\end{aligned}
$$




$$
\mathrm{pH} \text { 9: }-\mathrm{r}_{O_{3}}=\mathrm{k} \mathrm{c}\left(\mathrm{O}_{3}\right)_{\mathrm{a}}
$$

Where $-\mathrm{r}_{O_{3}}$ is the ozone decomposition rate, $k$ is the constant of decomposition, $\mathrm{c}\left(\mathrm{O}_{3}\right)_{\mathrm{a}}$ is the ozone concentration in water.

The order of ozone decomposition reaction rate changed from $3 / 2$ to 1 when $\mathrm{pH}$ changed from 3 to 9 in the above equations, but it does not mean that the decomposition rate decreased when the $\mathrm{pH}$ increased. As Beltran (1995) reported, the reaction constant increased with $\mathrm{pH}$ dramatically (the rate constant of the ozone decomposition reaction was found to be $8.3 \times 10^{-5} \mathrm{sec}^{-1}$ at $\mathrm{pH} 2$, the rate constant was $2.1 \mathrm{sec}^{-1}$ at $\mathrm{pH}$ 12). While the $\mathrm{c}\left(\mathrm{O}_{3}\right)_{\mathrm{a}}$ did not drop as much as rate constant increased, $\mathrm{c}\left(\mathrm{O}_{3}\right)_{\mathrm{a}}$ was $9.96 \times 10^{-6} \mathrm{M}$ at $\mathrm{pH} 2$, and $\mathrm{c}\left(\mathrm{O}_{3}\right)_{\mathrm{a}}$ was $2.23 \times$ $10^{-6} \mathrm{M}$ at $\mathrm{pH} 12$, as shown in Figure 2.4 of Section 2.3.2.2. Thus the ozone decomposition rate increased with $\mathrm{pH}$ increased.

As shown in Table 2.5, the reported reaction orders of ozone decay by different researchers were listed.

Table 2.5: A comparison of reported reaction orders for the decay rate of ozone in phosphate buffered solutions of demineralized water (Gottschalk et al., 2000)

\begin{tabular}{lllc}
\hline Reference & $\mathrm{T}$ in $^{\circ} \mathrm{C}$ & $\mathrm{pH}$ & $\mathrm{n}$ with respect to $\mathrm{O}_{3}$ \\
\hline Stumn, 1954 ${ }^{2)}$ & $0.2-19.8$ & $7.6-10.4$ & 1 \\
Kilpatrick et al., 1956 ${ }^{2)}$ & 25 & $0-6.8$ & 2 \\
& & $8 .-10$ & 2 \\
Rankas, 1962 ${ }^{1)}$ & $5 .-25$ & $5.4-8.5$ & 2 \\
Hewes and Davis, 1971 ${ }^{2)}$ & & $2 .-4$ & 2 \\
& $10 .-20$ & 6 & $1.5-2.0$ \\
Kuo et al., 1977 & & 8 & 1 \\
Sullivan, 1979 ${ }^{1)}$ & $15 .-35$ & $2.2-11$ & 2 \\
Gurol and Singer, 1982 & $3.5-60$ & $0.5-10$ & 1 \\
Staehelin and Hoigne, 1982 & 20 & $2.2-9.5$ & 2 \\
Sotelo et al.,, 1987 & 20 & $8 .-10$ & 1 \\
Minchews et al., 1987 & $10 .-40$ & $2.5-9$ & $1.5-2$ \\
Grasso and Weber, 1989 & & 7 & 2 \\
Gottschalk, 1997 & 20 & 6 & 1 \\
\hline 1) Taken from Gurol and Singer, 1982 ${ }^{2)}$ Taken from Minchown, 1987
\end{tabular}




\subsubsection{Equations in ozone decomposition}

In a small bubble columns or mechanically agitated semi-continuous tanks where a gas mixture $\left(\mathrm{O}_{2}-\mathrm{O}_{3}\right)$ is continuously fed into a volume of buffered water of a given $\mathrm{pH}$, set the gas and water phases are perfectly mixed, according to the hypothesis of perfect mixing, a molar balance of ozone in the water leads to the following equation (Beltran F., 2004):

$$
\frac{d c\left(O_{3}\right)_{a}}{d t}=G_{O_{3}}
$$

Where $c\left(\mathrm{O}_{3}\right)_{\mathrm{a}}$ is the ozone concentration in water, $G_{O_{3}}$ is the generation rate term of ozone, which varies depending on the kinetic regime of ozone absorption.

According to Beltran (2004), this equation can be described as:

$$
\frac{d c\left(O_{3}\right)_{a}}{d t}=k_{L} a\left[c^{*}\left(O_{3}\right)_{a}-c\left(O_{3}\right)_{a}\right]-k_{1} c\left(O_{3}\right)_{a}
$$

Where $k_{L a}$ and $k_{l}$ are the volumetric mass-transfer coefficient through the water phase and the rate constant of the ozone reaction, respectively, $\mathrm{c}^{*}\left(\mathrm{O}_{3}\right)_{\mathrm{a}}$ is the ozone solubility, also is the interface concentration.

Figure 2.2 shows a typical profile of the concentration of ozone with time for an absorption experiment in a semi-batch well-agitated tank, the concentration of dissolved ozone increases with time until it reaches a stationary value, at this time, $\frac{d c\left(O_{3}\right)_{a}}{d t}=0$, thus

$$
k_{L} a\left[c^{*}\left(O_{3}\right)_{a}-c\left(O_{3}\right)_{a}\right]=k_{1} c_{O_{3}}
$$

Therefore

$$
c\left(O_{3}\right)_{a}=\frac{k_{L} a c^{*}\left(O_{3}\right)_{a}}{\left(k_{L} a+k_{1}\right)}
$$




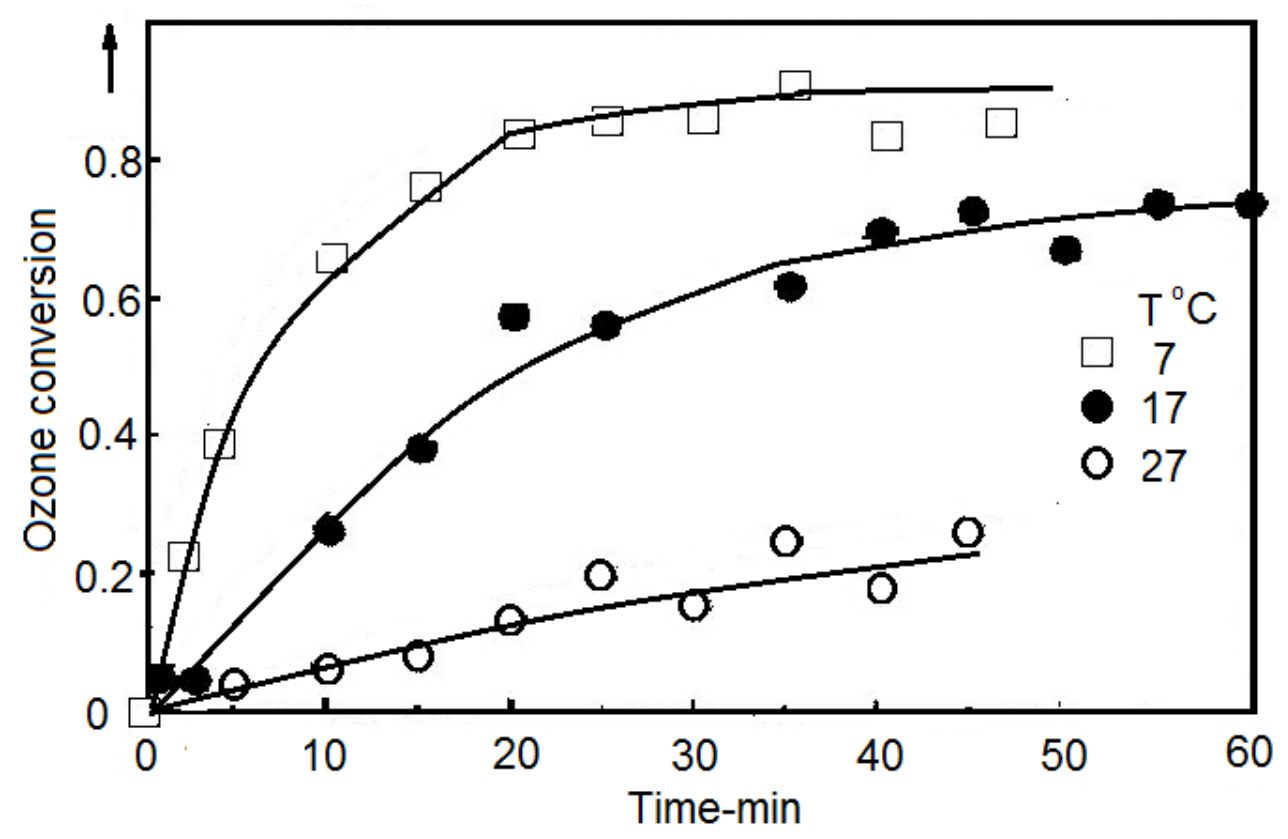

Fig 2.2 Typical concentration profiles of ozone against time obtained in ozone absorption in water at different temperature (Beltran, 2004)

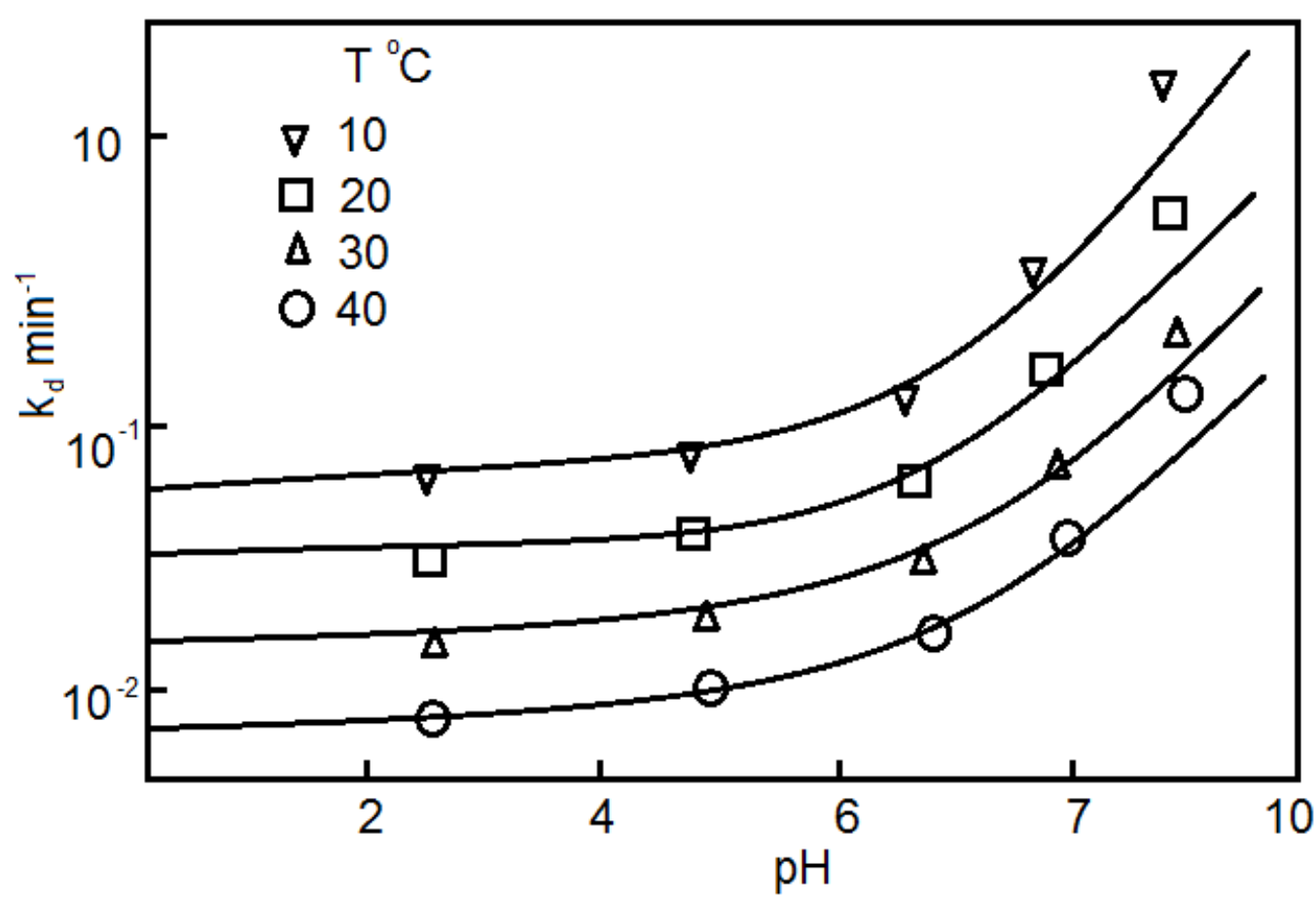

Fig 2.3 Variation of the apparent pseudo first-order rate constant of ozone decomposition in buffered distilled water with pH (Sotelo et al., 1987) 
$\mathrm{pH}$ is one of the main factors that influence the decomposition of ozone in water. As a general rule, for $\mathrm{pH}<7$ this variable has a slight effect on the ozone decomposition, but at higher $\mathrm{pH}$, the rate increases significantly. Figure 2.3 shows the variation of the apparent rate constant of the decomposition of ozone with $\mathrm{pH}$ in a study where first-order kinetics was considered.

Figure 2.4 shows an ozone concentration through a film layer. From experiments on ozone decomposition in water carried out at $\mathrm{pH} 2$ and $\mathrm{pH} 7$, the rate constant of the ozone decomposition reaction was found to be $8.3 \times 10^{-5} \mathrm{sec}^{-1}$ and $4.8 \times 10^{-4} \mathrm{sec}^{-1}$, respectively. When $\mathrm{pH}$ is higher, the rate constant is higher, such as at $\mathrm{pH} 12$, the rate constant is $2.1 \mathrm{sec}^{-1}$ (Beltran, F., 1995), for a direct reaction between ozone and the hydroxyl ion in organic -free water. It can be found from Figure 2.4 that the ozone concentration is lower in water when the $\mathrm{pH}$ is higher, the results for the case of low mass transfer $\left(k_{L}=2 \times 10^{-5} \mathrm{sec}^{-1}\right)$.

Beltran F. in 1995 also determined the reaction and the diffusion times for the ozone decomposition reaction from data on the rate constants at different $\mathrm{pH}$ values and the mass-transfer coefficient, In Figure 2.5 the reaction time of the ozone decomposition is plotted at different $\mathrm{pH}$ showing the zone where the kinetic regime is slow or fast. It is deduced that at a $\mathrm{pH}$ lower than 12 , the ozone decomposition reaction will not interfere with the direct ozone reactions of fast or instantaneous kinetic regime. On the contrary, at a $\mathrm{pH}$ higher than 12 , the ozone decomposition reaction will be the only way ozone disappears when the ozone direct reactions of compounds present in water. 


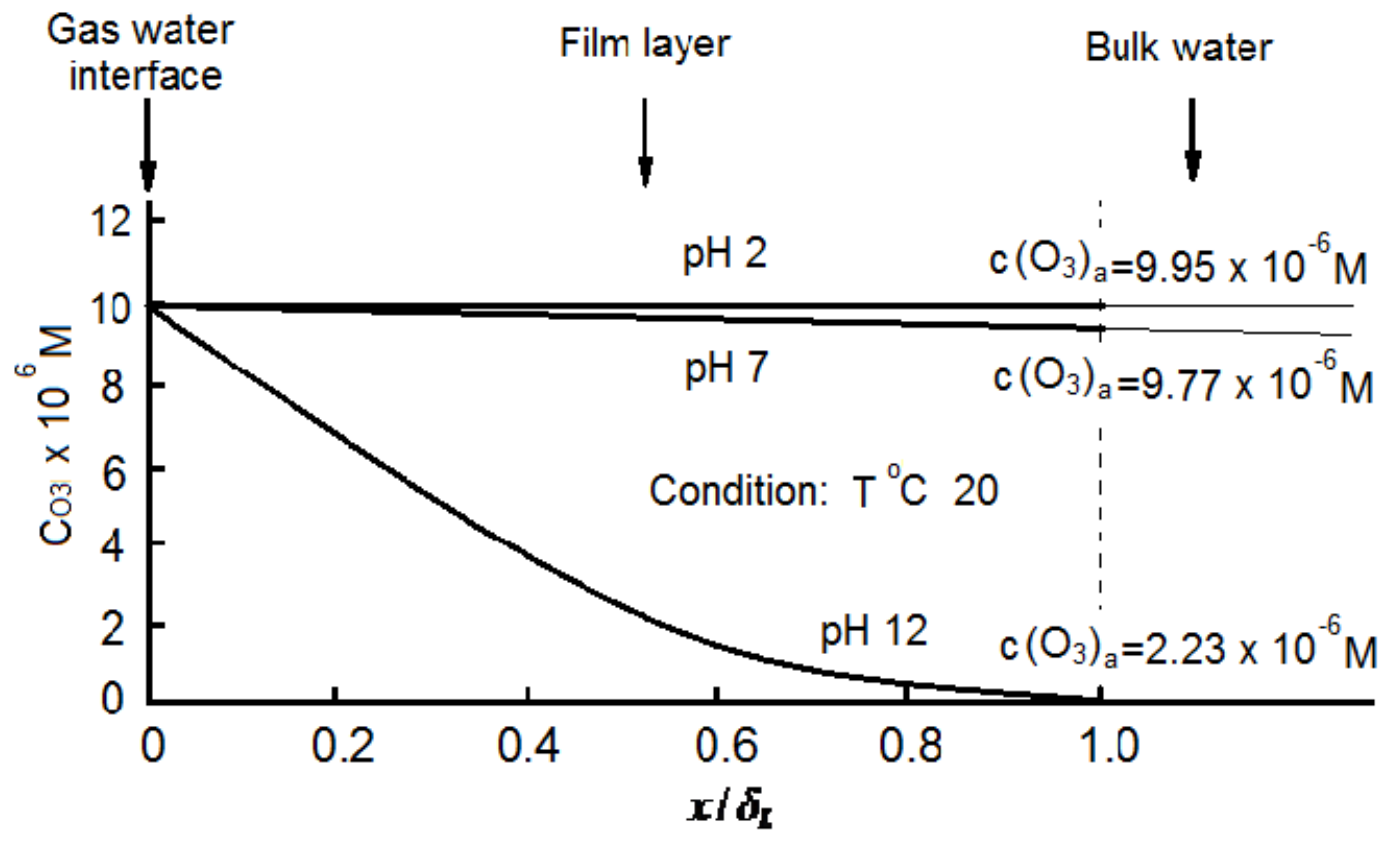

Fig 2.4 Variation of the concentration of ozone with the depth of liquid penetration during its absorption at steady state, $\mathrm{x} / \delta_{\mathrm{L}}$-depth of film layer, $\mathrm{c}\left(\mathrm{O}_{3}\right)_{\mathrm{a}}$-ozone concentration in bulk layer (Beltran, 1995).

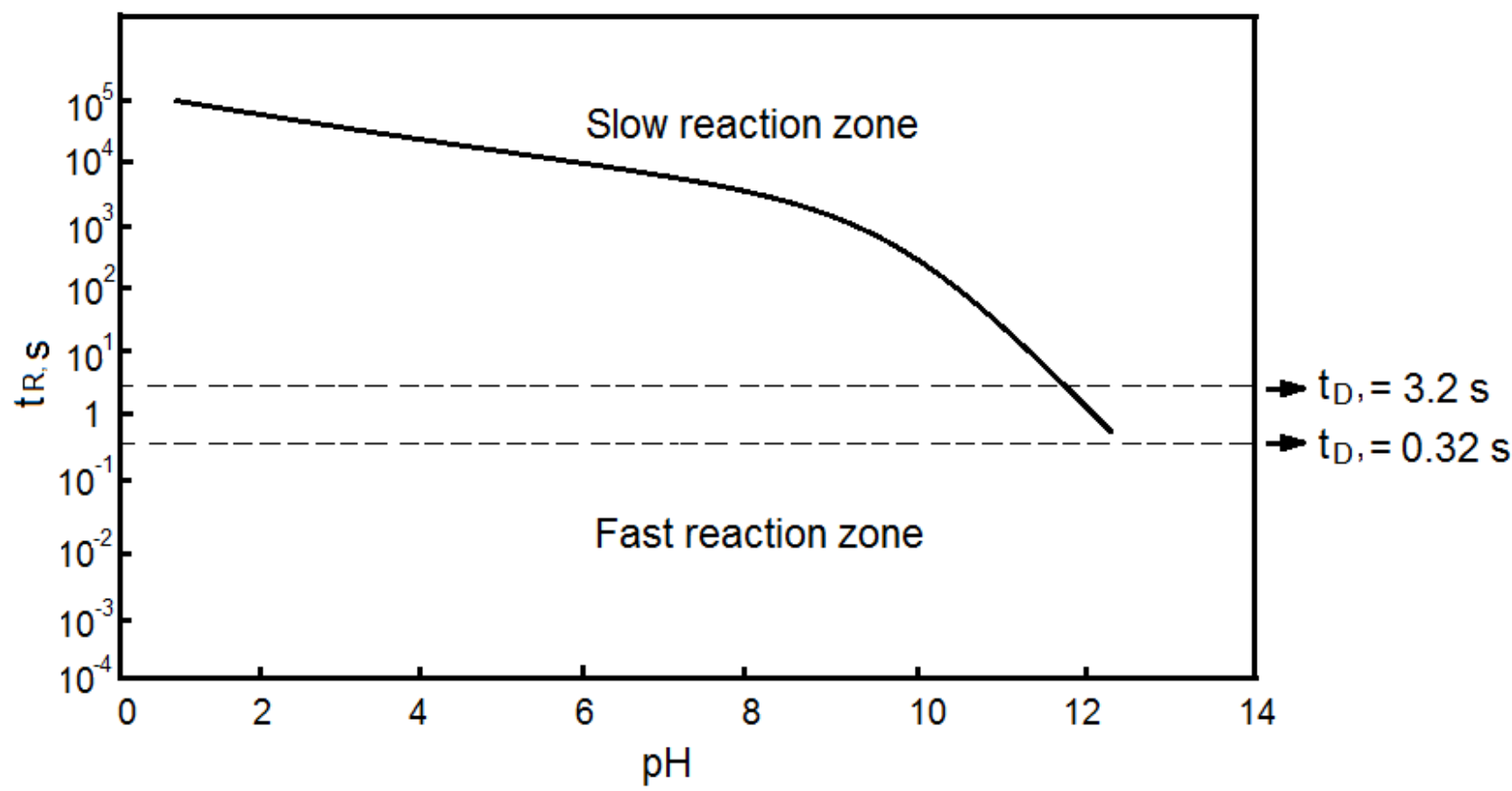

Fig 2.5 Reaction time evolution of the ozone decomposition reaction with $\mathrm{pH}$ at $20^{\circ} \mathrm{C}$ (Beltran, 1995), $t_{D}$-the diffusion time, $t_{R^{-}}$the reaction time, $s$-time (second). 


\subsection{Mass Transfer of Ozone}

Mass transfer of ozone from gas to water in the presence and absence of chemical reactions, and determination of the mass transfer coefficient are discussed in this section.

\subsubsection{Mass transfer of ozone in two phases}

\subsubsection{Equilibrium concentration for ozone}

As shown in Figure 2.6, when the controlling resistance is in the liquid phase, the over-all mass transfer coefficient $K_{L a}$ is general used

$$
\mathrm{m}=k_{g a}\left(c_{g^{-}} c_{g i}\right)=k_{L a}\left(c_{L i}-c_{L}\right)=K_{L a}\left(c^{*} L^{-} c_{L}\right)
$$

where: m-mass transfer rate of ozone and $\mathrm{c}_{\mathrm{L}}^{*}$ is the liquid concentration in equilibrium with the bulk gas concentration.

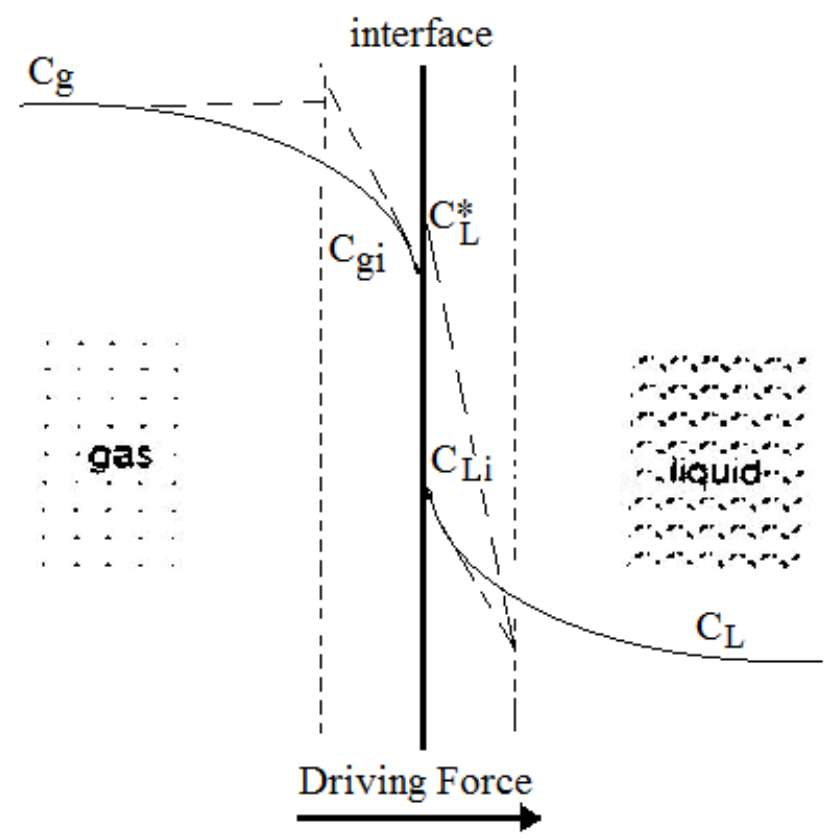

Fig 2.6 Gas dissolves into liquid through a boundary (Gottschalk et al., 2000) 
$\mathrm{N}_{\mathrm{Ao}}=-\mathrm{D}_{\mathrm{A}} \frac{d c_{A}}{d x}=\frac{D_{A}}{\delta_{L}}\left(c^{*}{ }^{-} c_{L}\right)$

$\mathrm{N}_{\mathrm{Ao}}$ is the mass transfer rate of ozone, i,e. $\mathrm{N}_{\mathrm{Ao}}=\mathrm{m}$.

$K_{L a}=\frac{D_{A}}{\delta_{L}}$

where $\delta_{L}$ is the film thickness, the characteristic of film theory.

For dilute non-reacting solutions, Henry's Law is used to describe the linear equilibrium distribution of a compound between the bulk liquid and gas phases

$\mathrm{H}_{\mathrm{c}}=\frac{c_{g}-c_{g i}}{c^{*}{ }_{L}-c_{L i}}=\frac{c_{g i}-c^{*}{ }_{g}}{c_{L i}-c_{L}}$

$\mathrm{H}_{\mathrm{c}}=$ dimentionless Henry's Law constant. $c_{g}$ and $c_{g i}$ are concentration of gas component in gas mixture, $c_{L}, c_{L i}$ are concentration of gas component in liquid.

\subsubsection{Mass transfer with simultaneous chemical reactions}

An enhancement factor $\mathrm{E}$ has been defined to describe the increase in mass transfer due to a simultaneous reaction:

When the mass transfer is liquid side control, $\frac{1}{K_{L a}} \approx \frac{1}{k_{L a}}$, then

$\mathrm{E}=\left[\frac{\text { rate for mass transfer with reaction }}{\text { rate for mass transfer alone }}\right]=\frac{m_{r}}{k_{L a}\left(c^{*}{ }_{L}-c_{L}\right)}$

$\mathrm{m}_{\mathrm{r}}=E \cdot k_{L a}\left(c^{*}{ }^{-}{ }^{-} C_{L}\right)$ 


\subsubsection{Determination of mass transfer coefficients of continuous-flow model}

According to Figure 2.7, the general mass balance for aqueous phase at nonsteady state, considering convection, mass transfer and reaction (e.g. ozone dacay), can be written (Gottschalk et al., 2000, p96):

$V_{L} \frac{d c_{L}}{d t}=Q_{L}\left(c_{L o}-c_{L}\right)+k_{L a} V_{L}\left(c_{L}^{*}-c_{L}\right)-r_{L} V_{L}$

The integrated form of the equation without reaction $\left(r_{L}=0\right)$ is (Gottschalk et al., 2000):

$$
\ln \left[1-\frac{c_{L}-c_{L o}}{c_{L \infty}-c_{L o}}\right]=-\mathrm{K}_{2} \cdot \mathrm{t}
$$

where $\mathrm{K}_{2}=\frac{Q_{L}}{V_{L}}+k_{L a}, c_{L o}=$ ozone concentration at $\mathrm{t}=0$, and $c_{L^{\infty}}=$ ozone concentration at $\mathrm{t}$ $=\infty$.

For the liquid phase (continuous - flow):

$$
k_{L a}=\frac{Q_{L}}{V_{L}} \frac{\left(c_{L}-c_{L o}\right)-r_{L} V_{L}}{c^{*}{ }_{L}-c_{L}}
$$

When diffusion, convection, and chemical reaction proceed simultaneously, based on gas absorption theory, the microscopic mass-transfer equation of A applied in liquid phase, the mass transfer is described as Equation (2-27),

$$
\mathrm{D}_{T} \nabla^{2} \mathrm{c}_{A}=\mathrm{U} \nabla \mathrm{c}_{A}+\frac{\partial c_{A}}{\partial t}
$$


With chemical reaction

$\mathrm{D}_{T} \nabla^{2} \mathrm{c}_{A}+\mathrm{r}_{A}=\mathrm{U} \nabla \mathrm{c}_{A}+\frac{\partial c_{A}}{\partial t}$

This is the case of ozonation reaction (Beltran F., 2004).

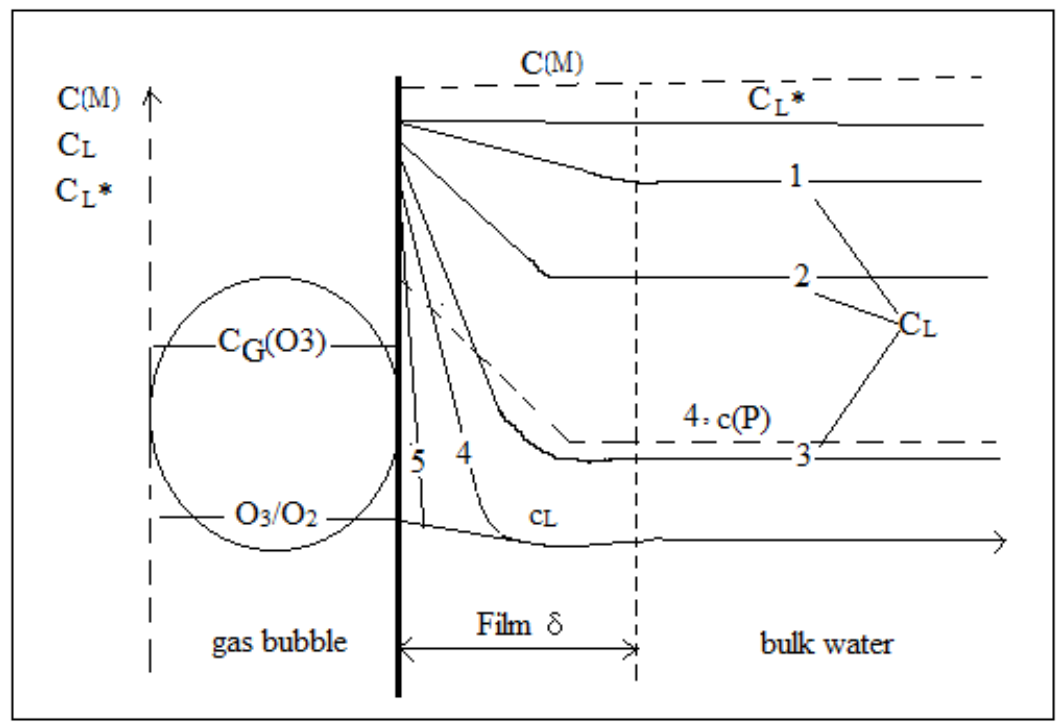

Fig 2.7 A gas-liquid reacting system (Gottschalk et al., 2000)

\subsubsection{Determination of the gas-liquid dispersion parameters $\left(d_{32}, \varepsilon G\right.$, a and $\left.U_{S}\right)$}

L'opez et al. (2007) investigated gas-liquid dispersion with the images treatment (fast photo taking in short interval). It was found that when a gas is dispersed in a liquid, the bubble shape was not rigorously spherical but always slightly ellipsoidal. Yet, the phenomenon never appeared to be very remarkable. The diameter of the equivalent disc $\left(d_{i}\right)$ of each individual bubble was estimated assuming a spherical shape. Finally, the Sauter diameter $\left(d_{32}\right)$ was calculated from Eq. (2-29) for each experimental condition. The gas hold-up $(\varepsilon \mathrm{G})$ was obtained using Eq. (2-30) by measuring the still liquid $\left(h_{\mathrm{L}}\right)$ and the gas-liquid dispersion $\left(h_{\mathrm{G}}\right)$ heights. From these results, Eq. (2-31) was used to calculate the interfacial area $(a)$. The linear rising velocity of an individual bubble (Us) was determined according to Eq. (2-32), where $D$ was the distance covered by the bubble between two 
successive video pictures ( $\left.t_{\text {images }}\right)$. Assuming a perfectly quiescent liquid phase, this velocity is also the slip velocity $\left(U_{\mathrm{S}}\right)$ of the bubble. This procedure of images acquisition and treatment was repeated for each gas flow rate (L'opez et al., 2007).

$\mathrm{d}_{32}=\frac{\sum n_{i} d_{i}^{3}}{\sum n_{i} d_{i}^{2}}$

$\varepsilon G=1-\frac{h_{L}}{h_{G}-L}$

$a=\frac{6 \varepsilon G}{d_{32}(1-\varepsilon G)}$

$\mathrm{Us}=\frac{\Delta D}{\Delta t_{\text {image }}}$

\subsubsection{Simultaneous determination of mass transfer parameters $\left(k_{\mathrm{La}}, \mathbf{k}_{\mathrm{C}}\right.$ and $\left.\mathrm{m}\right)$}

An experiment of ozone dissolution in pure water typically led to the concentration profiles at the gas outlet and in the liquid phase shown in Figure 2.8, where the unsteady state and the steady state periods have been separated. Once the steady state has been reached, $\left[\mathrm{O}_{3}\right]_{\mathrm{G} \text {,out }}$ which is the unsteady ozone out concentration, takes the particular value $\left[\mathrm{O}_{3}\right]^{\infty}$ G,out which is the steady ozone out concentration; and $\left[\mathrm{O}_{3}\right]_{\mathrm{L}}$ which is the actual ozone concentration in water, takes the particular value $\left[\mathrm{O}_{3}\right]_{\mathrm{L}}^{\infty}$ which is the steady ozone concentration in water. The equilibrium concentration $\left[\mathrm{O}_{3}\right]_{\mathrm{L}}^{*}$ of dissolved ozone, always higher than the actual concentration $\left[\mathrm{O}_{3}\right]_{\mathrm{L}}$, is also reported in Figure 2.8. Once the steady state has been reached, the mass balance for ozone dissolution in the gas phase of the reactor reduces to Eq. (2-33) where $G$ is the volume gas flow rate and $V_{\mathrm{L}}$ is the liquid volume (L'opez et al., 2007). Accounting that the gas hold-up was very low in the experimental conditions investigated here- the liquid and total reactor volumes have been considered as equal. This equation was used to determine the kinetic constant $\left(k_{\mathrm{C}}\right)$. 
The mass balance for ozone dissolution in the liquid phase is presented in Eq. (2-34). Its integration over the unsteady state period provided the expression of the volumic ozone transfer coefficient $\left(k_{\mathrm{L} a}\right)$, which is presented in Eq. (2-35). Moreover, the solution for the steady state period allows writing of the solubility parameter (S) according to Eq. (2-36) where an average concentration for the gas phase was used. Considering that the gas phase behaves like in a plug flow reactor, this average value corresponds to Eq. (2-37) (L'opez et al., 2007).

$\mathrm{k}_{\mathrm{C}}=\frac{G\left(\left[O_{3}\right]_{G, \text { in }}-\left[O_{3}\right]_{G, \text { out }}^{\infty}\right)}{\left[O_{3}\right]_{L}^{\infty} V_{L}}$

$\frac{d\left[O_{3}\right]_{L}}{d t}=k_{L a}\left[O_{3}\right]_{L}^{*}-\left(k_{L a}+k_{C}\right)\left[O_{3}\right]_{L}$

$\ln \left(\left[\mathrm{O}_{3}\right]_{L}^{\infty}-\left[\mathrm{O}_{3}\right]_{\mathrm{L}}\right)=-\left(\mathrm{k}_{\mathrm{La}}-\mathrm{k}_{\mathrm{C}}\right) \mathrm{t}+\ln \left[\mathrm{O}_{3}\right]_{L}^{\infty}$

$\mathrm{S}=\frac{\left[O_{3}\right]_{L}^{*}}{\overline{\left[O_{3}\right]_{G}}}=\frac{\left[O_{3}\right]_{L}^{\infty}}{\overline{\left[O_{3}\right]_{G}^{\infty}}} \frac{k_{L a}+k_{C}}{k_{L a}}$

${\overline{\left[O_{3}\right.}}_{G}=\frac{\left[O_{3}\right]_{G, \text { in }}-\left[O_{3}\right]_{G, \text { out }}}{\ln \left(\left[O_{3}\right]_{G, \text { in }} /\left[O_{3}\right]_{G, \text { out }}\right)}$

where $\overline{\left[\mathrm{O}_{3}\right]}$ is the average gas ozone concentration. 


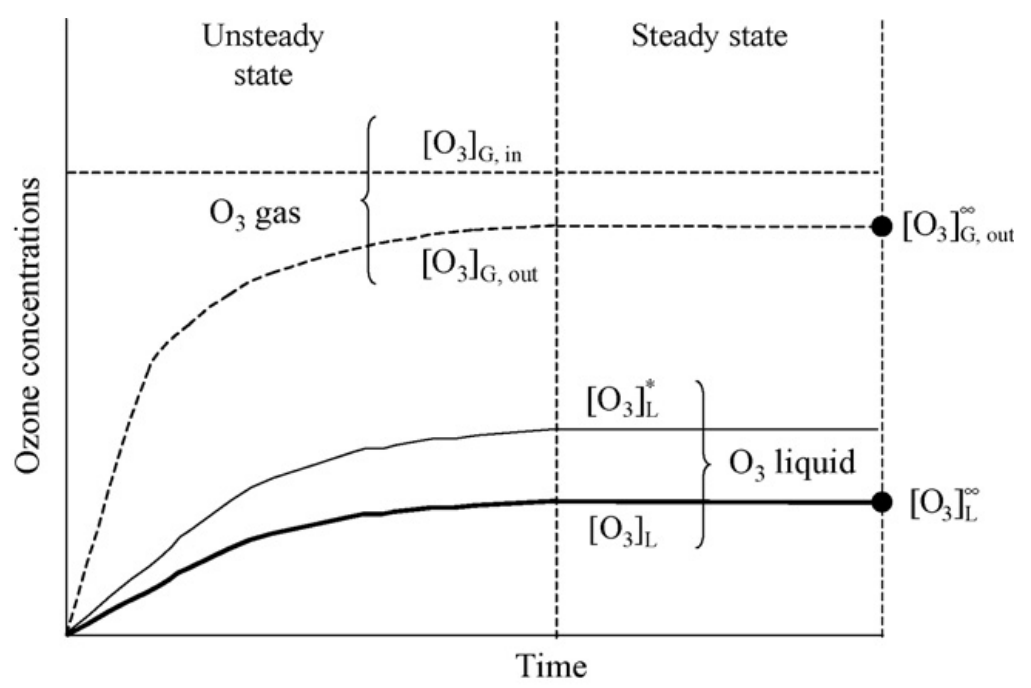

Fig 2.8 Typical ozone concentration profiles (L'opez et al. 2007)

\subsection{Ozonation Kinetics and Scavengers}

In this section, ozone reaction in pure water or aqueous phase, and ozone reaction with polyethylene were discussed.

\subsubsection{Standard potentials}

The standard potentials of water and ozone in $\mathrm{pH}<7$ solutions are shown in Fig 2.9.

The conversion of the new radical into a stable product completes the chain breaking process. However, this radical, by taking part in another reaction, may also provide a new chain path.

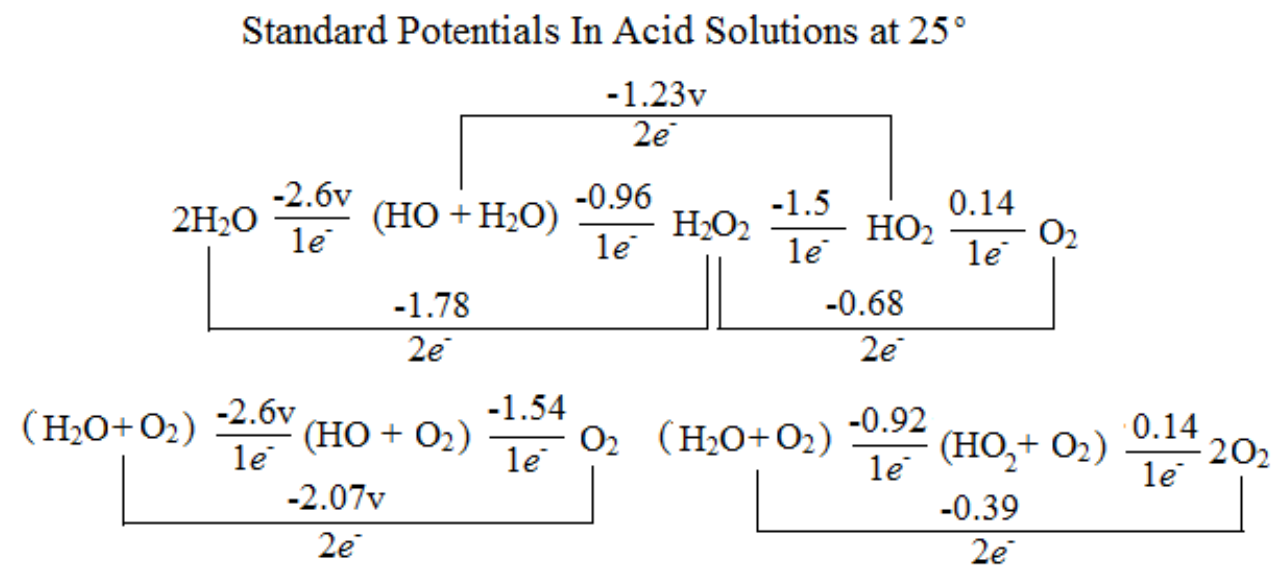

Fig 2.9 Standard potentials of ozone in acidic solution (Taube H. et al., 1940) 


\subsubsection{Reaction kinetics of ozone in pure water}

In water, ozone may react directly with substances, or it may decompose to form secondary oxidants such as $\cdot \mathrm{OH}$ radicals, which then themselves immediately react with substances, as shown in Figure 2.10. These different pathways of reactions lead to different oxidation products, and they are controlled by different types of kinetics. Therefore, their relative importance must be known when the oxidation effects of ozone and the rate of ozone consumption are to be predicted or generalized. Early observations of the lifetime of aqueous ozone indicated that the decomposition of the ozone becomes accelerated by increasing the $\mathrm{pH}$ (as discussed in Section 2.3.2). In addition, the decomposition of ozone at a given $\mathrm{pH}$ is often accelerated by a radical-type chain reaction which in non-pure solutions can be initiated, promoted, or inhibited by various materials, as shown in Figure 2.11. Because of the interaction of multiple pathways, the empirical kinetic laws describing the rate of ozone depletion deduced by different authors disagree even with respect to the kinetic orders of the reactions. Therefore a research program was started to separate the kinetics of the successive individual reaction steps and to investigate their interdependency. In a preliminary study the decomposition of ozone was analyzed in pure water and in aqueous solutions in which radical-type chain reactions were inhibited by $\cdot \mathrm{OH}$ radical scavengers such as bicarbonate. Because $\cdot \mathrm{OH}$ and $\cdot \mathrm{O}_{2}$ were the assumed intermediates, then the reactions of these species with ozone were studied by producing them independently using pulse radiolyses. As shown in Figure 2.11, it was noticed that those organic materials which are known to convert $\cdot \mathrm{OH}$ radicals into $\mathrm{HO}_{2}$ - always accelerate the decomposition of ozone unless an ample amount of other radical scavengers is present (Hoigne et al., 1985).

\subsubsection{Initiation step}

The reaction between $\mathrm{OH}^{-}$ions and ozone leads to the formation of one superoxide anion $\left(\cdot \mathrm{O}_{2}{ }^{-}\right)$and one hydroperoxyl radical $\left(\mathrm{HO}_{2} \cdot\right)$ which are in an acid-base equilibrium (pK $=4.8$ ). For this initiation a rate constant was determined for free radical formation of $2 \times 70$ $\mathrm{M}^{-1} \mathrm{~s}^{-1}$ whereby the factor 2 is due to the fact that two radicals are produced per reaction. A comparable rate constant was found by Forni et al. 1982 when measurements were made in the high $\mathrm{pH}$ range. In addition, substance $\mathrm{M}$ may react with ozone and thereby either 
consume the ozone by a direct reaction or produce an ozonide ion radical by an electron transfer.

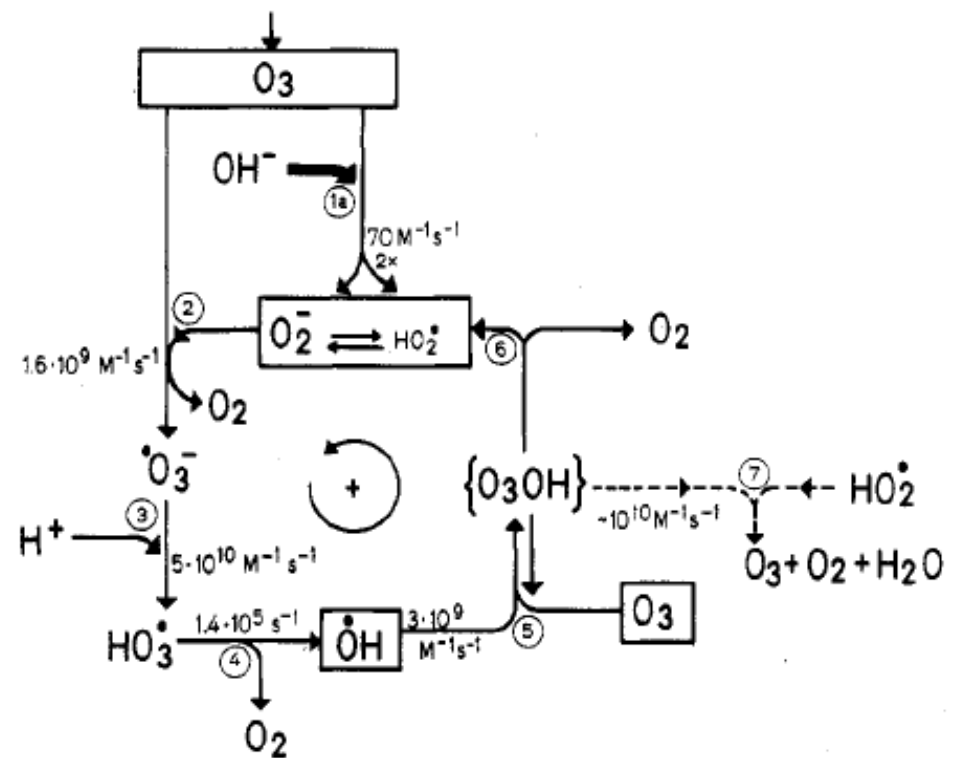

Fig 2.10 Reactions of aqueous ozone in "Pure Water" (Hoigne et al., 1985)

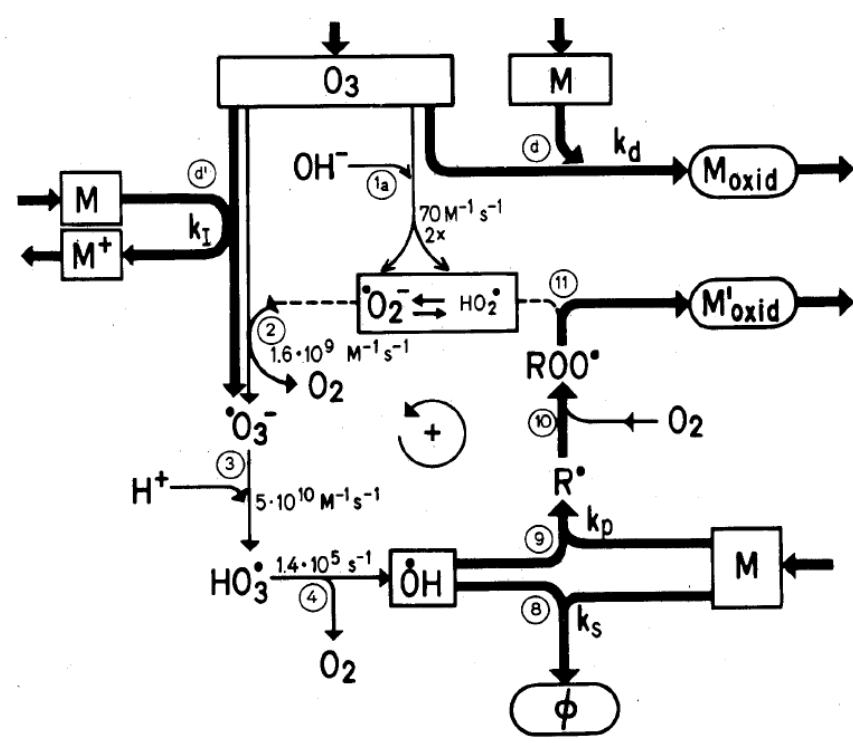

Fig 2.11 Reactions of aqueous ozone in the presence of materials $\mathrm{M}$ which react with $\mathrm{O} \cdot$, or which interact with $\cdot \mathrm{OH}$ radicals by scavenging and/or converting $\cdot \mathrm{OH}$ into $\mathrm{HO}_{2}$. (Hoigne et al., 1985).

\subsubsection{Propagation step}

Upon protonation $\cdot \mathrm{O}$, decomposes into $\cdot \mathrm{OH}$ radicals. These now react with substance 
M. Typical rate constants for reactions of $\cdot \mathrm{OH}$ with organic materials are in the range $10^{8}-10^{10} \mathrm{M}^{-1} \mathrm{~s}^{-1} \cdot \cdot \mathrm{OH}$ even reacts with $\mathrm{HCO}_{3}{ }^{-}$and $\mathrm{CO}_{3}{ }^{2-}$ with a rate constant of $2 \times 10^{3} \mathrm{M}^{-1} \mathrm{~s}^{-1}$ and $4 \times 10^{8} \mathrm{M}^{-1} \mathrm{~s}^{-1}$, respectively. Some functional groups present in organic molecules $\mathrm{M}$ are known to react with $\cdot \mathrm{OH}$ to form an organic radical which adds $\mathrm{O}_{2}$ and then eliminates $\mathrm{HO}_{2} \cdot \cdot \mathrm{O}_{2}{ }^{-}$in a base-catalyzed reaction. The rate constant with which the $\cdot \mathrm{O}_{2}^{-}$formed reacts with additional ozone is very high when compared with that of its (slow) reactions with other materials possibly present in ozonation. Therefore, the conversions of the less ozone selective $\cdot \mathrm{OH}$ radical into the highly selective $\mathrm{HO}_{2} \cdot$ promote the chain reaction.

\subsubsection{Termination step}

Many other organic and inorganic substrates react with $\cdot \mathrm{OH}$ radicals to form such secondary radicals which do not predominantly produce $\mathrm{HO}_{2} \% \cdot \mathrm{O}_{2}{ }^{-}$. The scavengers-the chemical groups (such as t-butyl alcohol, carbonate ion) which are capable of capturing free radicals from other chemical groups, generally terminate the chain reaction, as shown in Figure 2.12.

\section{Alkyl groups}

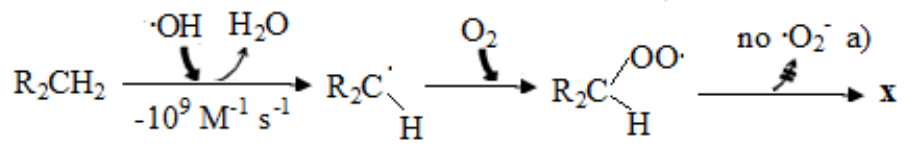

\section{D. t-Butyl alcohol}

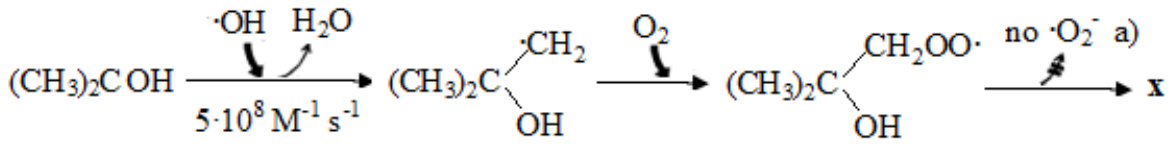

\section{E. Bicarbonate and carbonate ion}

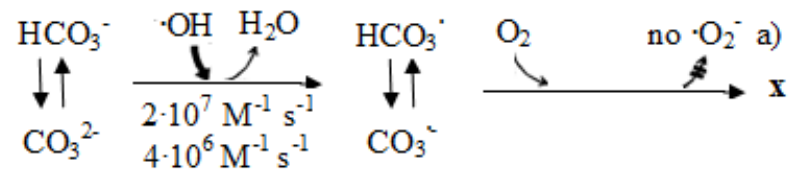

Fig 2.12 Scavengers to capture the $\cdot \mathrm{OH}$ radicals (Hoigne et al., 1985) 


\subsubsection{Other hypothesis of ozonation in the aqueous phase}

Figure 2.13 showed the scheme of reactions of aqueous ozone. Ozone decomposed in the aqueous phase generated radicals $\cdot \mathrm{OH}$ and $\cdot \mathrm{O}_{2}{ }^{-}$; and reacted with substrate $\mathrm{M}$ to form $\mathrm{M}_{\text {oxid. }}$. Generated radicals reacted with substrate $\mathrm{M}^{\prime}$ to form $\mathrm{M}^{\prime}{ }_{\text {oxid }}$, or were captured by scavengers.

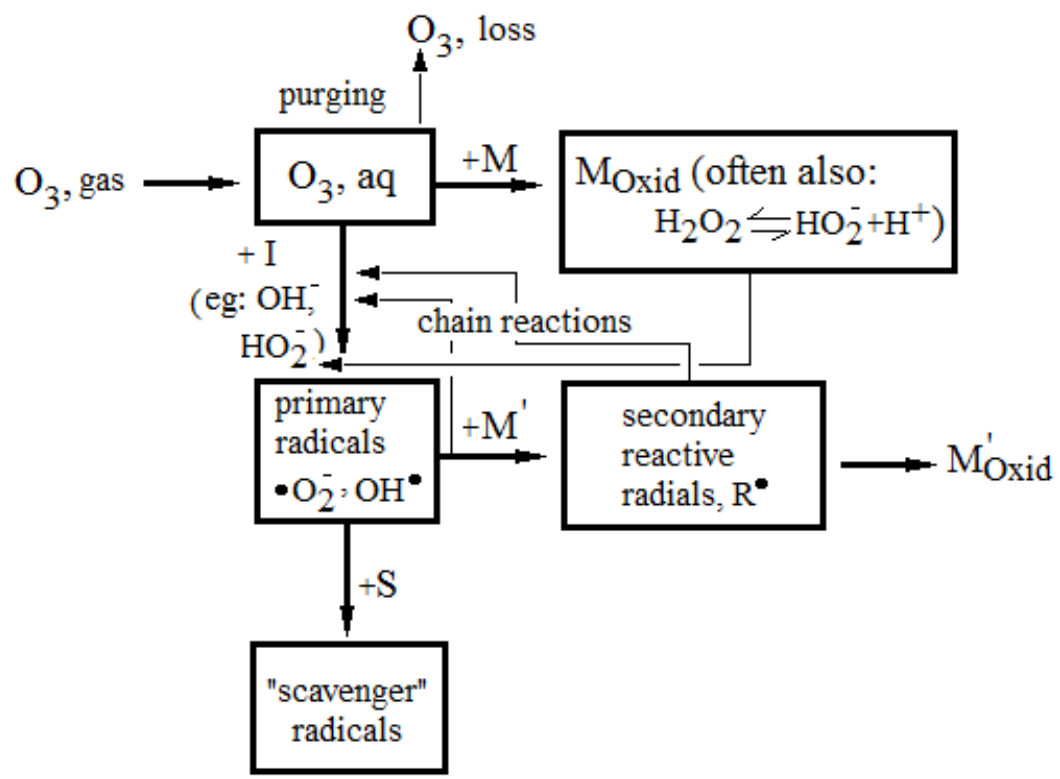

Fig 2.13 Scheme of reactions of aqueous ozone. M, materials that consume ozone and become oxidized to $\mathrm{M}_{\text {oxid }}\left(\right.$ and $\mathrm{H}_{2} \mathrm{O}_{2}$ ); I, materials that initiate the decomposition of ozone to primary radicals; $\mathrm{S}$, free radical scavenger; $\mathrm{M}^{\prime}$, materials that react with $\mathrm{OH}^{\cdot}$ and form secondary radicals $\mathrm{R}$. and become finally oxidized to $\mathrm{M}^{\prime}{ }_{\text {oxid }}$ (Staehelln et al., 1982)

Figure 2.14 showed the half-lives of ozone vs. concentration of carbonate for different $\mathrm{pH}$ values decreased with the higher $\mathrm{pH}$ value. 


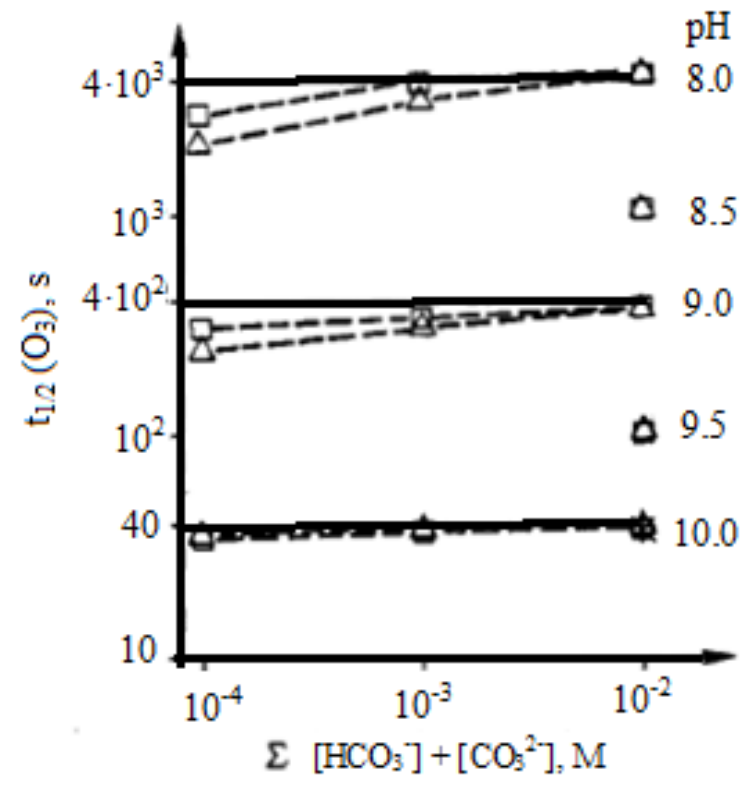

Fig 2.14 Successive half-lives of ozone vs. concentration of carbonate for different $\mathrm{pH}$ values. $\left[\mathrm{O}_{3}\right]_{0}=50 \mu M$ : 1st $\mathrm{t}_{1 / 2}, \Delta ; 2 \mathrm{nd} \mathrm{t}_{1 / 2}, \square .\left[\mathrm{O}_{3}\right]_{\mathrm{o}}=3 \mu M$, (Staehelln et al., 1982)

Figure 2.15 showed relative concentration of ozone vs. time for different $\left[\mathrm{O}_{3}\right]_{\mathrm{o}}$ at high scavenger concentration: there were not much differences of $\left[\mathrm{O}_{3}\right] /\left[\mathrm{O}_{3}\right]_{\mathrm{o}}$ at $0.3 \mu M\left[\mathrm{O}_{3}\right]_{\mathrm{o}}$ to $12 \mu M\left[\mathrm{O}_{3}\right]_{\mathrm{o}}$ (Staehelln et al., 1982)

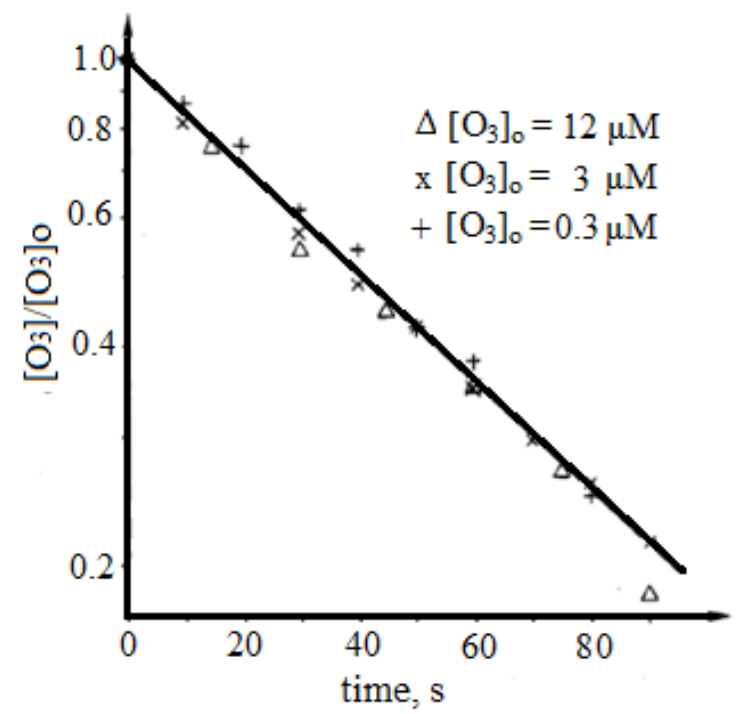

Fig 2.15 Relative concentration of ozone vs. time for different initial $\mathrm{O}_{3}$ concentrations at high scavenger concentration: $\left[\mathrm{HCO}_{3}{ }^{-}\right]+\left[\mathrm{CO}_{3}{ }^{2-}\right]=10 \mathrm{mM} ;\left[\mathrm{PO}_{4}{ }^{3-}\right]_{\text {total }}=50 \mathrm{mM} ; \mathrm{pH} 10.0$ (Staehelln et al., 1982) 
Even the orders of reaction reported in the literature for "pure water" were different. This result is not surprising since in earlier studies the rate of the decomposition in "pure water" was accelerated by undefined chain reactions subsequent to $\mathrm{OH}^{-}$attack. The rate constant $\mathrm{k}_{\mathrm{O} 3, \mathrm{OH}^{-}}$is small when compared with the rate constants quoted for other reactions of $\mathrm{O}_{3}$. This measured value is still different from the rate constant of the primary $\mathrm{OH}^{-}$attack on $\mathrm{O}_{3}$ molecules because it must be assumed that more than one $\mathrm{O}_{3}$ molecule is destroyed per primary initiation step. To get an estimation of the overall stoichiometry of the $\mathrm{O}_{3}+\mathrm{OH}^{-}$ reaction (when all $\mathrm{OH} \cdot$ radicals are scavenged before reacting with $\mathrm{O}$,) the following reaction sequences can be considered:

Hypothesis I (extended for dissociation of $\mathrm{HO}_{2}$ - and electron-transfer mechanism) (Staehelln J. et al., 1982)

$$
\begin{aligned}
& \mathrm{HO}_{2} \cdot \longrightarrow \mathrm{H}^{+}+\cdot \mathrm{O}_{2}{ }^{-} \quad \mathrm{pK}=4.8 \\
& \mathrm{O}_{3}+\cdot \mathrm{O}_{2}{ }^{-} \longrightarrow \mathrm{O}_{3}{ }^{-}+\mathrm{O}_{2} \\
& \cdot \mathrm{O}_{3}{ }^{-}+\mathrm{H}_{2} \mathrm{O} \longrightarrow \mathrm{OH}^{\cdot}+\mathrm{OH}^{-}+\mathrm{O}_{2} \\
& \mathrm{O}_{3}+\mathrm{OH}^{-} \longrightarrow \cdot \mathrm{HO}_{2}{ }^{-}+\mathrm{O}_{2} \\
& \mathrm{H}_{2} \mathrm{O}_{2} \longrightarrow \mathrm{HO}_{2}{ }^{-}+\mathrm{H}^{+} \quad \mathrm{pK},=11.6
\end{aligned}
$$

Hypothesis II (Staehelln J. et al., 1982)

$$
\mathrm{O}_{3}+\mathrm{HO}_{2}^{-} \longrightarrow \mathrm{OH} \cdot+\cdot \mathrm{O}^{-}+\mathrm{O}_{2}
$$

The occurrence of $\cdot \mathrm{O}_{2}{ }^{-}$as an intermediate (hypotheses I and II) is supported by qualitative chemical detections based on the reduction of tetranitromethane. For this experimental system the stoichiometric yield of $\mathrm{OH} \cdot$ radical formation was found to be 0.55 
\pm 0.08 . It is close to 0.67 as predicted by hypotheses I and II. Because the observed overall reaction has been found to be first order in $\mathrm{O}_{3}$ concentration, the sequences of reactions formulated by hypotheses I and II must both be assumed to be rate limited only by the initiation step. Likewise, $\cdot \mathrm{O}_{2}{ }^{-}$and $\cdot \mathrm{HO}_{2}{ }^{-}$can only exist as short-lived intermediates in the $\mathrm{pH}$ ranges here considered (Staehelln et al., 1982).

\subsubsection{Mechanism about peroxide generation of ozonation on polyethylene}

Razumovskii et al., 1971 did the investigation of gaseous ozonation on polyethylene powders. The reaction mechanism was suggested as follows:

Initial step:

$\mathrm{O}_{3}+\mathrm{PH} \stackrel{k_{l}}{\longrightarrow} \cdot \mathrm{OH}+\mathrm{POO} \cdot$

Propagation step:

$\mathrm{PH}+\mathrm{POO} \cdot \stackrel{k_{2}}{\longrightarrow} \mathrm{P} \cdot+\mathrm{POOH}$

$\mathrm{O}_{2}+\mathrm{P} \cdot \stackrel{k_{3}}{\longrightarrow} \mathrm{POO}$

$\mathrm{O}_{3}+\mathrm{POOH} \stackrel{k_{4}}{\longrightarrow} \mathrm{O}_{2}+\cdot \mathrm{OH}+\mathrm{POO} \cdot$

Termination step:

POO $\stackrel{k_{5}}{\longrightarrow}$ Inactive (carbonyl and carboxyl groups)

Combine equation 2.30 to 2.34 , the following expression was obtained (Kefeli A.A., et al., 1971):

$[\mathrm{POOH}]=\frac{k_{1} k_{2}[\mathrm{PH}]^{2}\left[O_{3}\right]}{\alpha k_{5}}\left(1-e^{-\alpha t}\right)$ 
Where $\alpha=k_{4}\left[O_{3}\right]\left\{1-\frac{k_{2}[P H]}{k_{5}}\right\}$

One of our researching group member did the catalytic ozonation to polyethylene film (Mastan et al., 2013), it was found that $[\mathrm{POOH}]$ on polyethylene film surface also had a exponential relationship with ozonation time.

\subsection{Kinetics of Membrane Flux Decline}

This section approaches to identify and mathematically describe fouling kinetics, including effects of pore blockage and cake formation; and focus on mitigation fouling by modification of the microstructure of membrane surfaces.

\subsubsection{Gas and liquid fouling model}

Naturally dissolved and colloidal organic matter (NOM), acting alone or in concert with inorganic ions and colloids, is considered a major contributor to membrane fouling in water treatment applications, including microfiltration, ultrafiltration and nanofiltration. NOM is a heterogeneous mixture of complex organic materials including humic substances, hydrophilic acids, proteins, lipids, carboxylic acids, amino acids, and hydrocarbons (Kilduff et al., 2005).

Flux decline caused by organic macromolecules, (acting alone or in concert with inorganic species, including inorganic colloids), may result from concentration polarization; from pore blockage by solute adsorbed on the membrane surface or within pores; and from the formation of a cake layer on the membrane surface, which presents a resistance to flow in addition to the membrane itself.

The molar trans-membrane flux for liquid

$$
\mathrm{Ni}=\frac{K_{i} D_{i}}{t_{M}}\left(\mathrm{C}_{F, i}-\mathrm{C}_{P, i}\right)
$$


Where $\mathrm{D}_{i}$ is the diffusivity of solute in the membrane and the product $\mathrm{K}_{\mathrm{i}} \mathrm{D}_{\mathrm{i}}$ is the permeate $\mathrm{P}_{\mathrm{Mi}}, \mathrm{C}_{F, i}$ and $\mathrm{C}_{P, i}$ are the pressure on the feed and permeate surface of the membrane (Max $\mathrm{S}$. et al., 2003).

The separation factor is defined as

$$
\mathrm{a}_{i, j}^{*}=\frac{y_{P, i}}{x_{R, i}} \frac{y_{P, j}}{y_{R, j}}
$$

where $\mathrm{y}$ is the mole fraction in the permeate leaving the membrane and $\mathrm{x}$ the mole fraction in the retention on the feed side of the membrane (Max S. et al., 2003), $i, j$ are the components in the liquid.

\subsubsection{Dead-end filtration fouling model}

For dead-end filtration under constant applied pressure, rate laws, which are corresponding to pore blockage, pore constriction, and cake formation, can be written by assuming that the number of pores, the pore diameter, or the mass of a cake layer formed at the membrane surface, respectively; and change in proportion to the convective transport of mass to the membrane surface. Various fouling models are obtained for each fouling mode, which can all be formulated as a single equation as shown by (Kilduff et al., 2005):

$$
\frac{d^{2} t}{d w^{2}}=k_{f}\left(\frac{d t}{d w}\right)^{n}
$$

where $t$ is time (s), $w$ is the mass filtered $(\mathrm{kg}), k_{f}$ is a fouling coefficient with units that depend on the value of $\mathrm{n}$ (e.g., $\mathrm{s} \mathrm{kg}^{-2}$, for $n=0$ ) and $n$ is a dimensionless filtration constant that reflects the mode of fouling: (i) cake formation corresponds to $n=0$; (ii) complete pore blocking corresponds to $n=2$; and, (iii) standard blocking (pore constriction) corresponds to $n=1.5$. 


\subsubsection{Combined pore blockage and cake filtration fouling model}

Ho and Zydney (2000) developed a model for dead-end filtration (UF and MF) of proteins that combines pore blockage and cake filtration modes of fouling. In this model, total flow through the membrane at any time during filtration, $\mathrm{Q}\left(\mathrm{kg} \mathrm{s}^{-1}\right)$, is the sum of flow through open pores (through which the resistance is $R m$ ) and through partially blocked pores on which a cake has formed, offering additional resistance to flow. In the absence of significant cross-flow, the combined pore blockage-cake filtration model is written (Kilduff et al., 2005):

$$
Q=Q_{o}\left[\exp \left(-\frac{\alpha_{b} \Delta P C_{f}}{R_{m}} t\right)+\frac{R_{m}}{R_{m}+R_{c}} \times\left(1-\exp \left(-\frac{\alpha_{b} \Delta P C_{f}}{R_{m}} t\right)\right)\right]
$$

where $\alpha_{b}$ is the pore blockage parameter $\left(\mathrm{m}^{2} \mathrm{~kg}^{-1}\right.$ solute); note that bulk or feed concentration, $C_{f}$, is in weight fraction units. The resistance from cake formation, $R c$, which increases as a function of time in proportion to the rate that NOM mass accumulates on the membrane surface, is obtained from:

$$
\frac{R_{m}+R_{c}}{R_{m}+R_{c, 0}}=\sqrt{1+\frac{2 \alpha_{c} \Delta P C_{f}}{\left(R_{m}+R_{c . o}\right)^{2}}} t
$$

where $\alpha_{c}\left(\mathrm{~Pa} \mathrm{~m}^{4} \mathrm{~s} \mathrm{~kg}^{-2}\right)$ is the cake formation parameter and $R c, o$ is the initial resistance of the deposit (i.e., a leakage flow).

Extensions to systems having significant cross flow were developed by Kilduff et al. (2002). This model is applicable when the resistance caused by concentration polarization is small, i.e., when salt rejection is negligible. The first term in Equation (2.43) is equivalent to the classical pore blockage model. This term dominates when $\mathrm{t}$ is small $\left(\mathrm{t}<<\mathrm{Rm} / \alpha \Delta \mathrm{PC}_{\mathrm{f}}\right)$ and the magnitude of the second term becomes negligible. At long time, $\left(t>>R m / \alpha \Delta \mathrm{PC}_{\mathrm{f}}\right)$ the second term dominates and the volumetric flux is governed by the classic cake filtration model. 


\subsection{Approaches to Mitigate Fouling}

A very important aspect of mitigate fouling is selecting a membrane material that minimizes attractive interactions between foulants and the membrane surface, e. g., hydrophilic modification of membranes.

\subsubsection{Surface modification}

Heterogeneous graft polymerization of specific functionalities or polymer layers has been employed as a route to tailor polymeric membrane surfaces for many different applications. In contrast to surface modification techniques such as alcohol wetting and surfactant adsorption, graft polymerization can modify membrane surface properties permanently. Applications have included filling membrane pores to improve selectivity of pervaporation membranes (Kai et al., 2000; Yamaguchi et al., 2001); attaching moieties to provide ion-exchange properties (Michel et al., 1998; Saito \& Yamashita, 1998; Kim \& Saito, 2000); and introducing affinity ligands for protein separations (Kiyohara et al., 1997). Considerable attention has been directed at using graft polymerization to increase the wettability of membrane surfaces as a route to reduce fouling.

A variety of hydrophilic monomers have been grafted to membrane surfaces to increase their hydrophilicity and reduce their potential to foul during filtration. Graft polymerization requires the generation of active ionic or radical sites on the membrane surface.

\subsubsection{Chemical schematic of graft polymerization}

As shown in Figure 2.16, polypropylene reacted with ozone and peroxide was generated on the backbone molecules. The peroxide decomposed when heated and created carbon radicals. These radicals initiated monomers with vinyl groups to graft onto the backbone by breaking the double bond; radicals transferred to the monomer carbon when the double bond broke, and the chain reaction continued to propagate; graft polymerization occurred till the radicals terminated. 

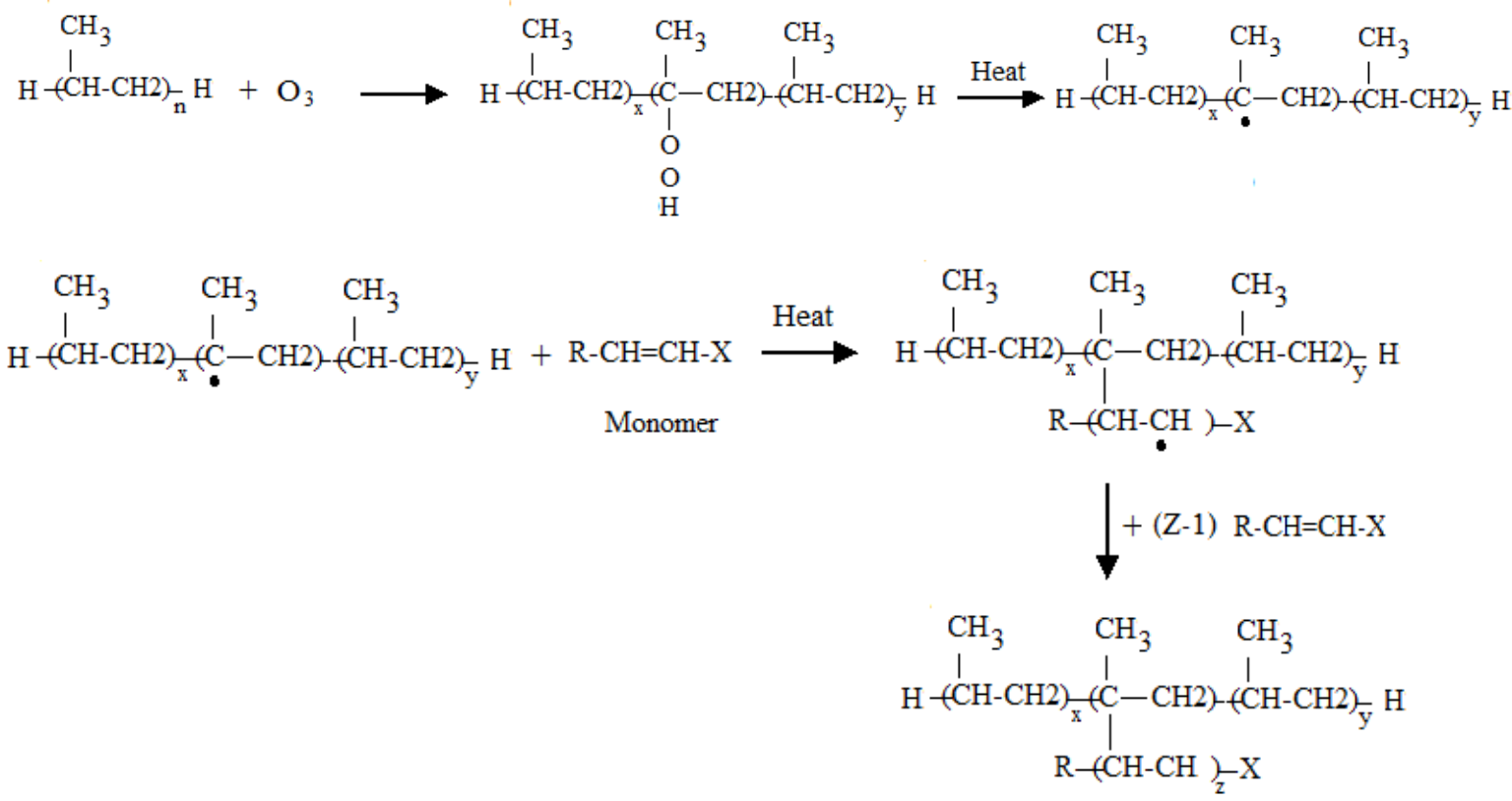

Fig 2.16 Radical graft polymerization

\subsubsection{Degree of grafting and grafting efficiency}

The hydrophilcility of modified membrane surfaces depends on monomer type, monomer concentration and active points of the substrates, all these factors govern the amount of polymer grafted to the surface. The degree of grafting achieved for a given ozonation dosage or UV lamp wavelength and energy dosage will depend on both the sensitivity of the base polymer, and intrinsic properties of the monomers employed. Proposed mechanism of UV-assisted graft polymerization of poly (aryl sulfone) membranes showing trunk polymer cleavage, formation of reactive radical sites, and chain growth (Yamagishi et al., 1995).

The grafting degree was calculated according to the following equation:

$$
\text { Grafting degree }(\text { wt. } \%)=\frac{w_{a}-w_{b}}{w_{b}} \times 100
$$

where $w_{\mathrm{b}}$ and $w_{\mathrm{a}}$ are the weight of a membrane before and after surface modification, respectively (Yu et al., 2007). 


\subsubsection{Effect of grafting on surface roughness and permeability}

Surface roughness may influence fouling, when the relative scale of the roughness and the size of solution components are similar, the fouling gets more (Xua et al., 2005).

Graft polymerization has been shown to reduce membrane permeability (increase membrane resistance). For example, a reduction in permeability is explained by pores blocked by grafted poly (vinyl pyrolidinone) (PVP) chains, and possibly some homopolymer deposition in pores. Controlling the graft degree and graft length is very important to obtain the desired hydrophilicity and avoid significantly affecting the permeation (Xua et al., 2005).

\subsection{Permeate Flux}

For a clean membrane, the method is based on the following relationship:

$$
J_{w}=\frac{\Delta P}{\mu_{0} R_{h}}
$$

where $J_{w}$ is the volumetric water flux, $R_{h}$ is the hydraulic resistance of the membrane, and $\mu_{0}$ is the viscosity of the permeate. However, for a fouled membrane, an additional resistance $R_{f}$ will be present, when passing (clean) water through the membrane:

$$
J_{w}=\frac{\Delta P}{\mu_{0}\left(R_{h}+R_{f}\right)}
$$

Thus, the values of $R_{f}$ can be determined, under specific (and similar) conditions for all the solutes in nonmodified and modified membranes, if $R_{h}$ is previously determined (when the membrane is clean), and this enables a comparison of the tendency of each solute to foul the membranes.

Permeate flux decline $(\%)=\left(1-\frac{J}{J_{0}}\right) \times 100$ 
where $J_{0}$ is the initial permeate flux taken at filtration time of $30 \mathrm{~min}$ and $J$ is the permeate flux at filtration time $t$ (Xua et al., 2005).

\subsection{Other Researchers' Work of Membrane Anti-fouling and Modification}

Works conducted by researchers previously on membrane fouling reduction and polymer surface modification were discussed as follows.

\subsubsection{Hydrophilic modification of polypropylene microfiltration membranes by ozone-induced graft polymerization}

Hydrophilic modification of polypropylene microfiltration membranes was performed with introduction of peroxide onto the membrane surface by gaseous ozone treatment followed by graft polymerization with hydroxyethyl methacrylate (HEMA), as shown in Figure 2.17. The HEMA grafting made the surface of the PP membrane hydrophilic and less adsorption to bovine serum albumin (BSA) proteins, and the results suggested that the protein fouling layer was reversible (means the fouling layer can be removed by washing or back flushing) because of the hydrophilic nature of the modified membrane (Wang et al., 1999).

Filtration of BSA proteins was conducted, the BSA solution has a concentration of $2000 \mathrm{mg} / \mathrm{l}$ and a pH 7.0 in 0.1 phosphate buffer was circulated through the membrane module. The permeate flux was measured using a balance (Wang et al., 1999).

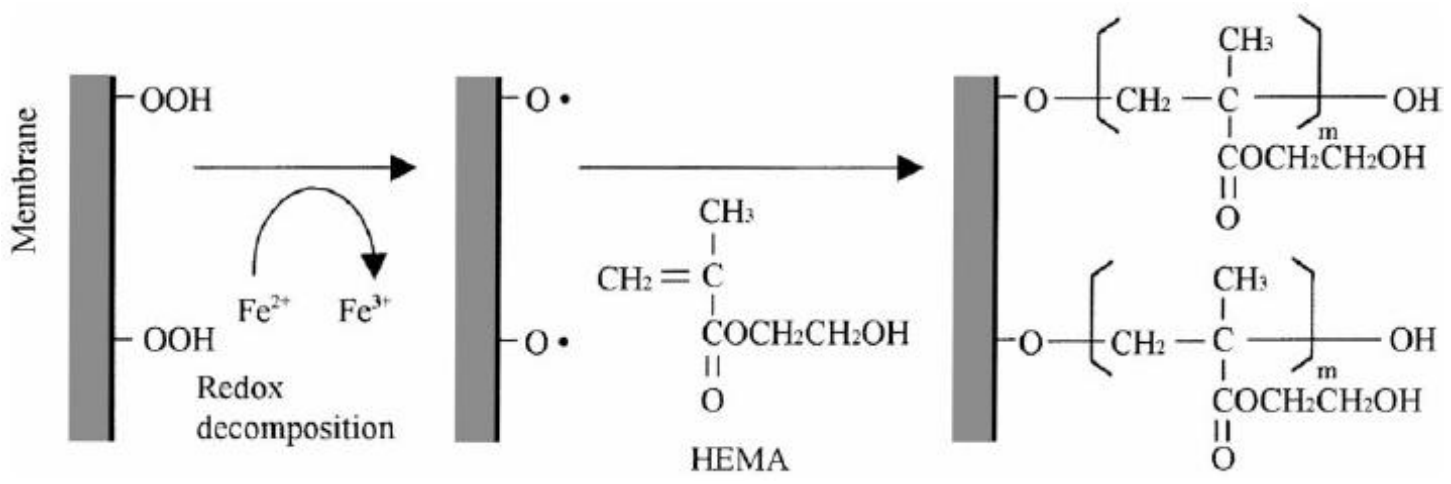

Fig 2.17 Schematic illustration of ozone-induced graft polymerization (Wang et al., 1999). 
Figure 2.18 showed the different initial flux of BSA solution through the modified and unmodified polypropylene membrane.

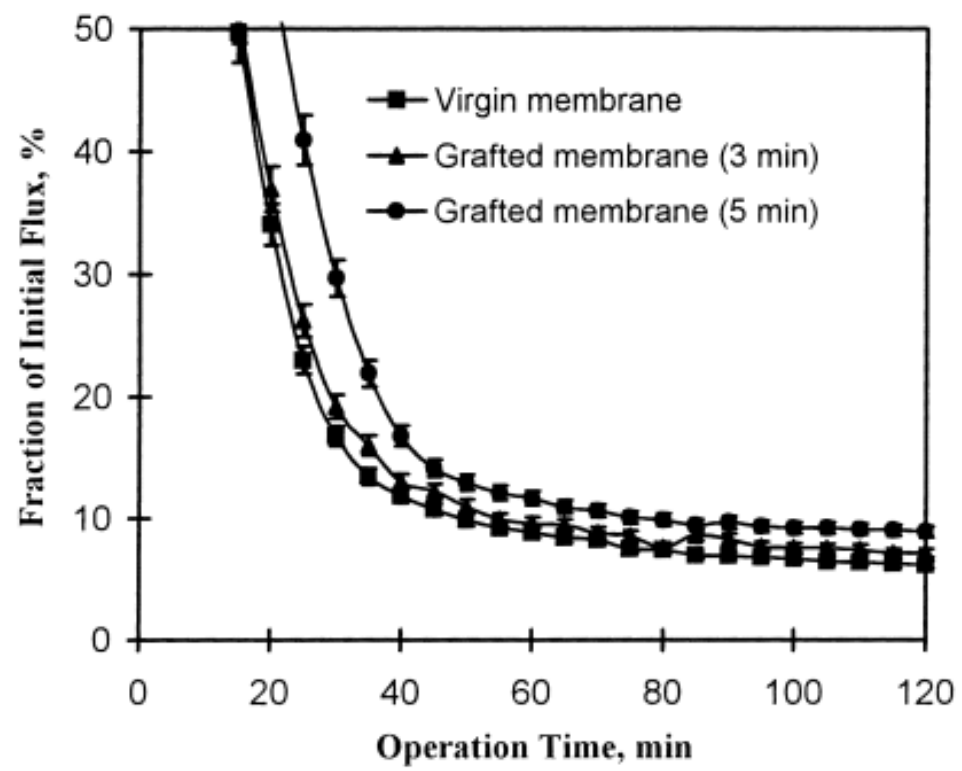

Fig 2.18 Fluxes for virgin and modified membranes during cross-flow MF of BSA solution (Wang et al., 1999)

As shown in Figure 2.19, the flux recovery efficiency that is closely associated with the reversibility of membrane fouling layers increased markedly with increasing the ozone treatment time by up to five minutes. The grafted membrane obtained with five minutes ozone treatment gave the highest flux recovery of approximately $95 \%$. However, the one minute ozonation had no effect on an improvement of flux recovery, which suggested that only part of the membrane surfaces and pores were modified during short time ozonation. It appears that membrane fouling might be caused by the accumulation of protein aggregates at the membrane surface regardless of the membranes used but could be reversed more easily for the grafted membranes with an appropriate time of ozonation. In summary, the protein fouling layer became reversible with grafting, being removed easily just by water flushing. This can be attributed to the more hydrophilic nature of the modified membrane surfaces. Also, the steric hindrance resulting from the poly-HEMA chains grafted may play a role in preventing the direct interaction between membrane surfaces and proteins (Wang et al., 1999). 


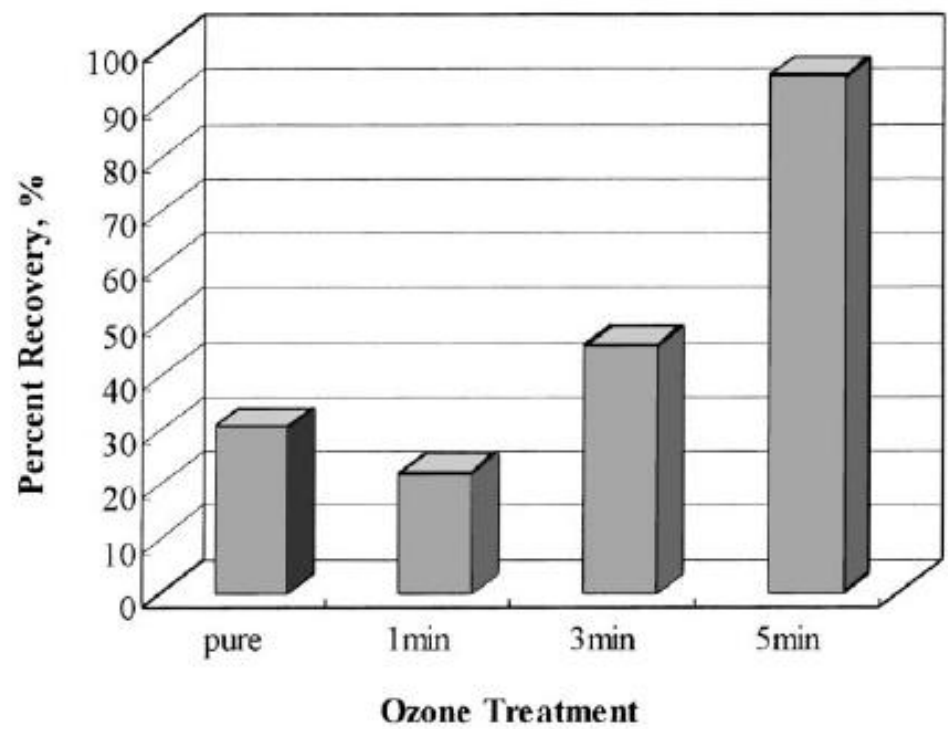

Fig 2.19 flux recovery efficiencies with water flushing at the end of operation (Wang et al., 1999)

\subsubsection{Membrane fouling reduction by back-pulsing and surface modification}

As shown in Figure 2.20, a combined method of back-pulsing and membrane surface modification was employed for the reduction of membrane fouling. A novel photo induced grafting method was used to render polypropylene (PP) membranes hydrophilic with neutral or positively or negatively charged surfaces by grafting monomers of poly(ethylene glycol 200) monomethacrylate (PEG200MA), dimethyl aminoethyl methacrylate (DMAEMA), or acrylic acid (AA), respectively. Both unmodified and modified PP membranes, as well as commercial cellulose acetate (CA) membranes, were evaluated in a cross-flow microfiltration system with and without back-pulsing in the presence of Escherichia coli bacterial suspensions. The permeate volume with back-pulsing is highest for the neutral, hydrophilically modified PP membrane and for the commercial CA membrane, approximately 2.6 times that of the unmodified PP membrane without back pulsing (Ma et al., 2000).

The base membranes used in the experiments are commercial porous disk PP microfiltration membranes with $47 \mathrm{~mm}$ diameter, $110 \mu \mathrm{m}$ thickness, $40 \%$ porosity, and $0.22 \mu \mathrm{m}$ nominal pore diameter and CA microfiltration membranes with $47 \mathrm{~mm}$ diameter, 120 
$\mu \mathrm{m}$ thickness, and $0.22 \mu \mathrm{m}$ nominal pore diameter.

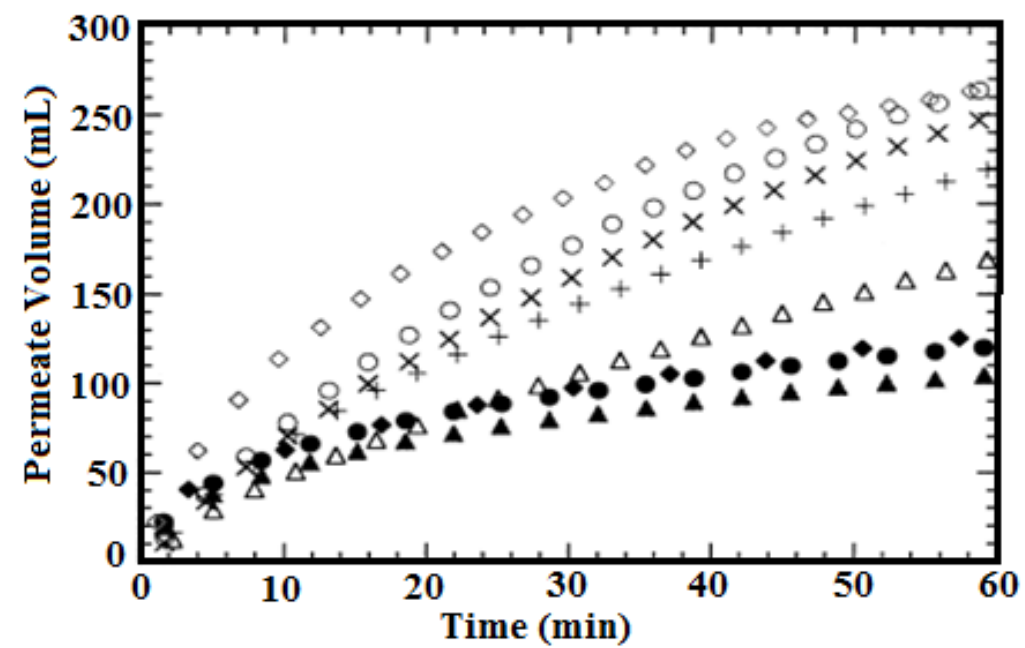

Fig 2.20 Permeate volume vs. filtration time for crossflow filtration of $0.14 \mathrm{~g} / 1 \mathrm{E}$. coli: $(\bigcirc),(\times),(+)$ backpulsing results for PP membranes modified with weight gains of $4.4 \mathrm{wt} . \%$ PEG200MA graft, 4.3 wt.\% AA graft, and 3.7 wt.\% DMAEMA graft, respectively; $(\Delta),(\boldsymbol{\Delta})$ unmodified PP membranes with and without backpulsing, respectively; (•) PP membrane modified with weight gain of 5.8 wt.\% PEG200MA and without backpulsing; $(\diamond),(\bullet$ commercial CA membranes with and without backpulsing, respectively. Backpulsing experiments were performed at a backward transmembrane pressure of 3.0 psi for $0.2 \mathrm{~s}$ after every $4 \mathrm{~s}$ of forward filtration at a transmembrane pressure of 5.0 psi (Ma et al., 2000).

\subsubsection{Polyolefins reacted with $\mathrm{O}_{3}$ in aqueous phase}

Dasgupta and his group (1990) investigated surface modification of polyolefins using ozone in aqueous phase for hydrophilicity and bondability. The polyolefin powder was dispersed in water becoming to pulp. The relationship between ozone uptake (percent) and the intrinsic viscosity of the polyolefin was studied. It was found that when the uptake increased, the intrinsic viscosity decreased; which meant that the molecular weight decreased and polymer chain scission had occurred. Figures 2.21 and 2.22 show the results.

On the other hand, carbonyl content (percent) increased. Figures 2.23 and 2.24 show the results.

The carbonyl and carboxyl contents generated with the ozone uptake were also 
studied, and were found to have increased with the ozone uptake percent.

The rate equation for the well-stirred batch reactor model may be expressed as:

$\frac{d X}{d t}=k\left(1-E^{\prime}\right)[\mathrm{O}]$

where $\mathrm{X}=$ ozone uptake, $\mathrm{g} \mathrm{O}_{3}$ consumed $/ \mathrm{g}$ pulp; $\mathrm{t}=$ time, min; $k=$ rate constant, liter gas $/ \mathrm{g}$ pulp min; $E^{\prime}=$ efficiency, $\mathrm{g} \mathrm{O}_{3}$ consumed / $\mathrm{g} \mathrm{O}_{3}$ in feed; and $[\mathrm{O}]=$ concentration of ozone in feed gas, $\mathrm{g} \mathrm{O}_{3} / \mathrm{L}$ gas at reaction temperature. Thus (1-E) $[\mathrm{O}]$ is the concentration of ozone in the gas leaving the reactor, which in the case of a well-stirred reactor model, is assumed to be equal to the concentration of ozone in the gas phase throughout the reactor [Dasgupta, 1990].

The rate constant was calculated from experimental data using the equation:

$$
k=\frac{X}{t\left(1-E^{\prime}\right)[O]}
$$




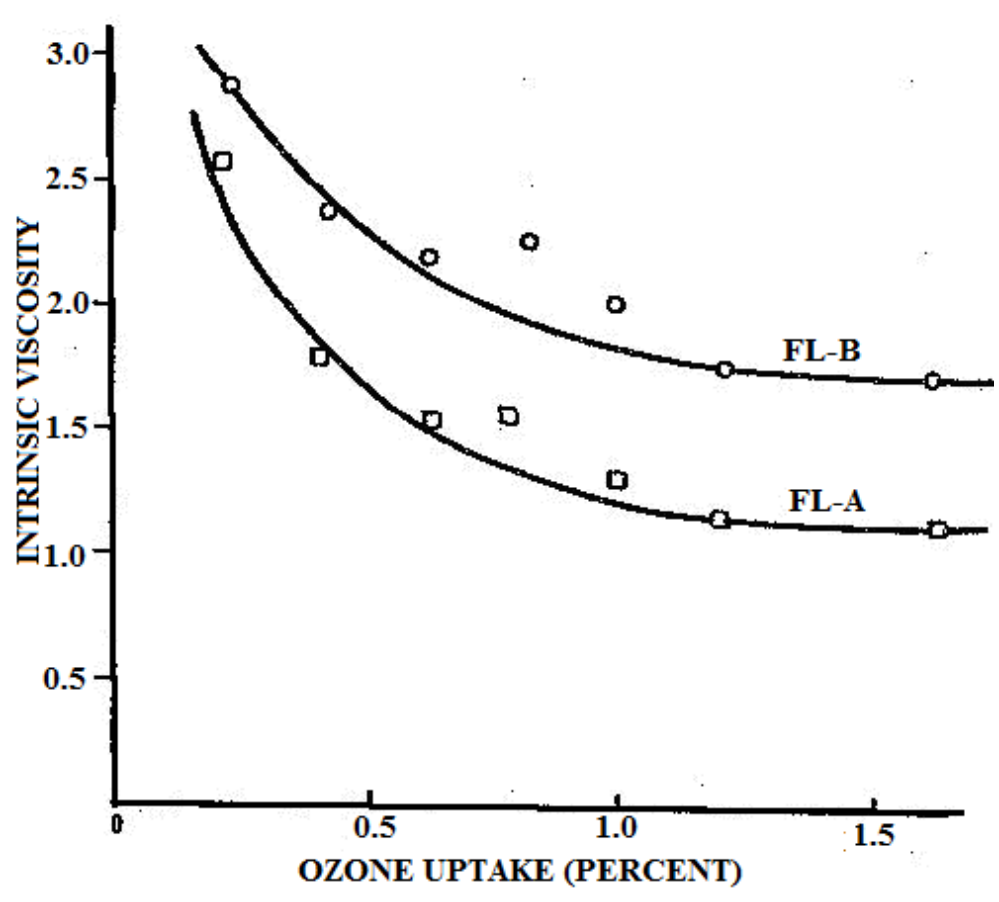

Fig 2.21 Intrinsic viscosity of polypropylene flake at various levels of ozone treatment. Flake FL-A and FL-B, which is FL-A-treated with a phenolic antioxidant stabilizer, are Hercules products. [Dasgupta, 1990]

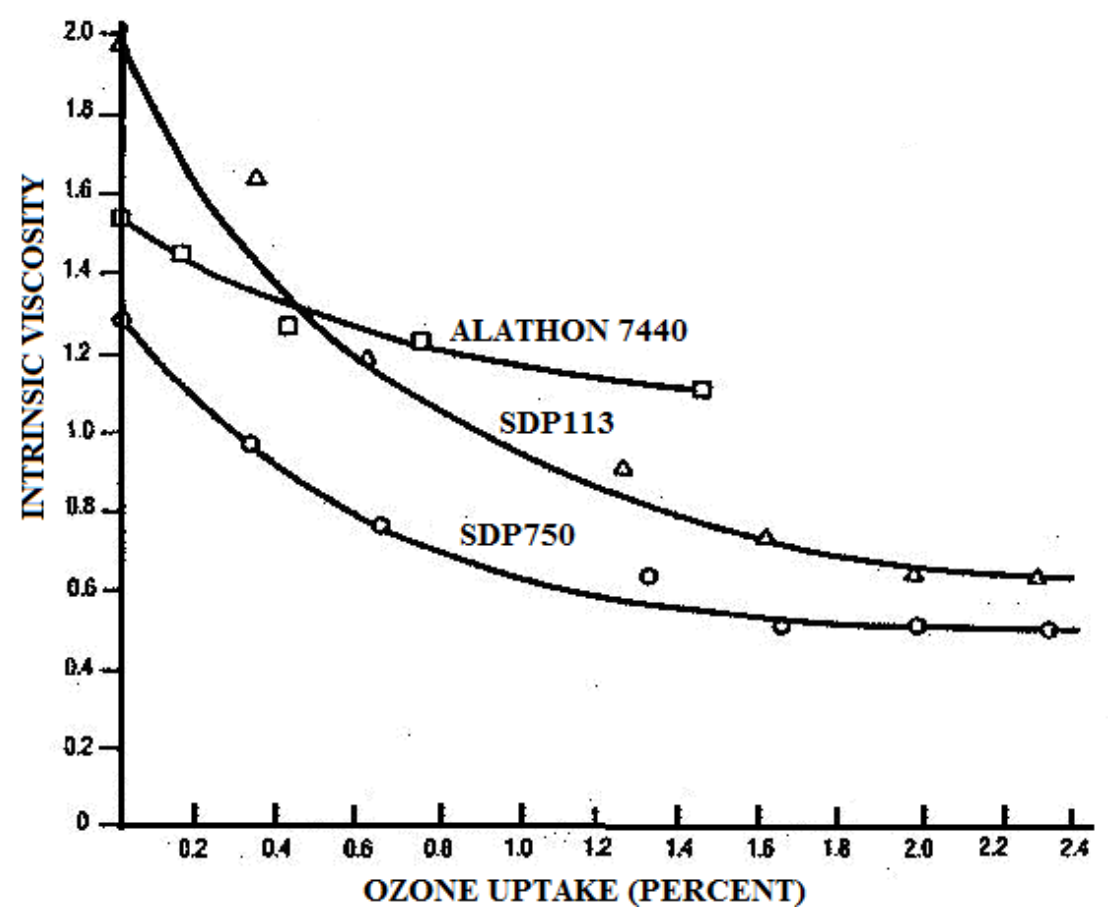

Fig 2.22 Intrinsic viscosity of polypropylene flake at various levels of ozone treatment. Alathon 7440 (Dupont) SPD-113 and SPD-750 (ARCO) have different molecular weights represented by intrinsic viscosity values [Dasgupta, 1990]. 


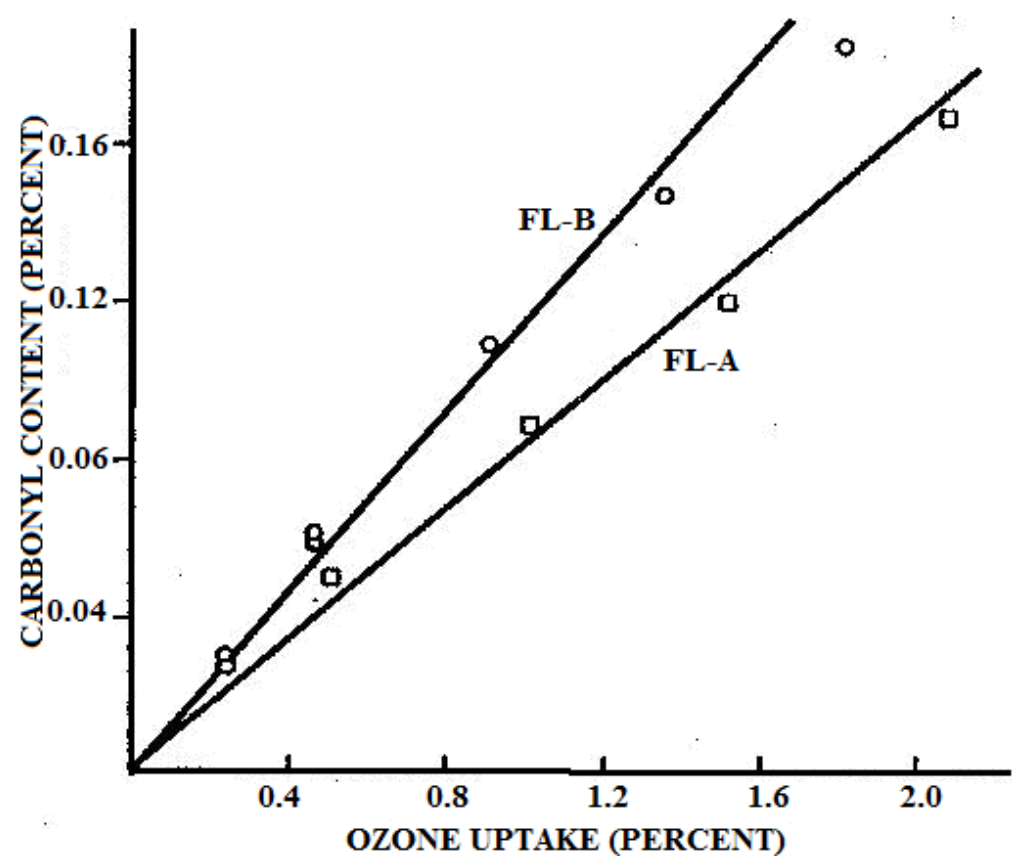

Fig 2.23 Carbonyl contents of ozonized stabilized and unstabilized polypropylene flake FL-B and FL-A respectively [Dasgupta, 1990].

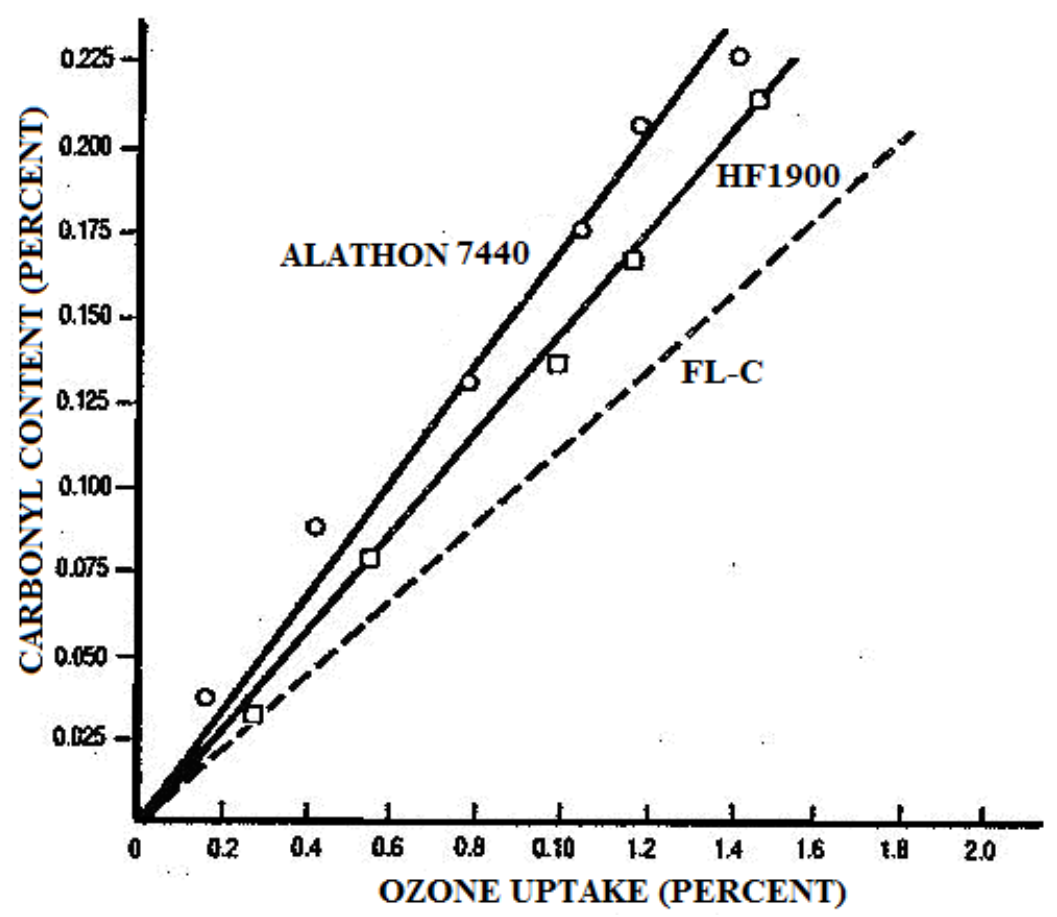

Fig 2.24 Carbonyl contents of ozonized polypropylene flake. Similar data for FL-C, a polypropylene flake provide a reasonable comparison [Dasgupta, 1990]. 


\subsubsection{BOPP reacted with $\mathrm{O}_{3}$ in gaseous and aqueous phases}

Figure 2.25 presents the results of the peroxide concentration generated. The results show that the peroxide content per area increased from $0.025 \mathrm{mmol} / \mathrm{m}^{2}$ to $0.148 \mathrm{mmol} / \mathrm{m}^{2}$ when the treatment time increased from $0.5 \mathrm{hrs}$ to $4.0 \mathrm{hrs}$ in aqueous solution, and increased from $0.044 \mathrm{mmol} / \mathrm{m}^{2}$ to $0.168 \mathrm{mmol} / \mathrm{m}^{2}$ when the treatment time increased from $1.0 \mathrm{hr}$ to 3.5 hrs in the gas phase. Peroxide content was generated slower in aqueous solution than in the gaseous phase after 2 hrs ozonation (Gu, 2008).

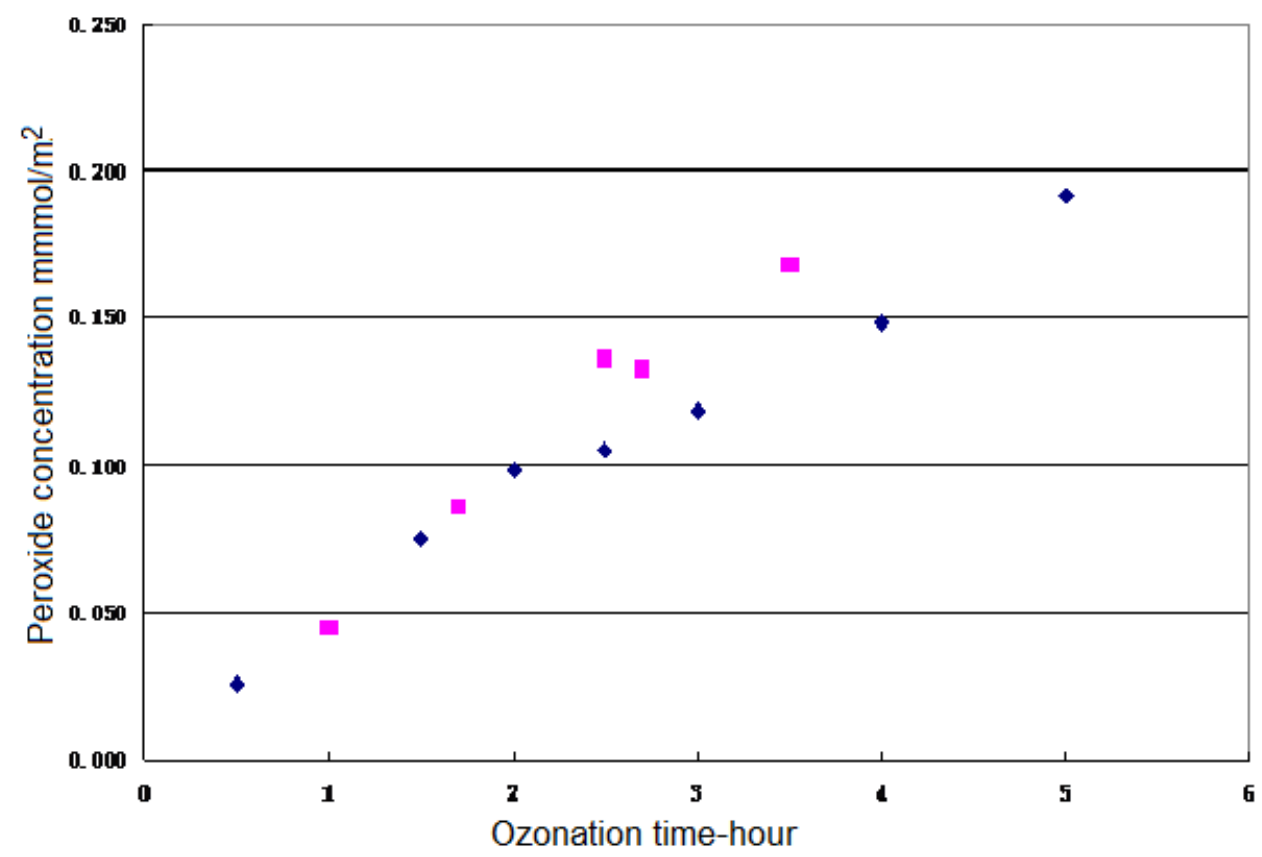

Fig 2.25 Peroxide generation of BOPP with $3.7 \mathrm{wt} \%$ ozone in: $\bullet$ aqueous phase, gaseous phase (Gu, 2008).

\subsubsection{List of other membrane anti-fouling modification}

As shown in Table 2.6, different methods and monomers had been used to modify the hydrophilicity of polymeric membranes and improve the anti-fouling. Ozonation is one of the methods, while the aqueous phase ozonation had not been found to be used. 
Table 2.6 List of other membrane anti-fouling modification

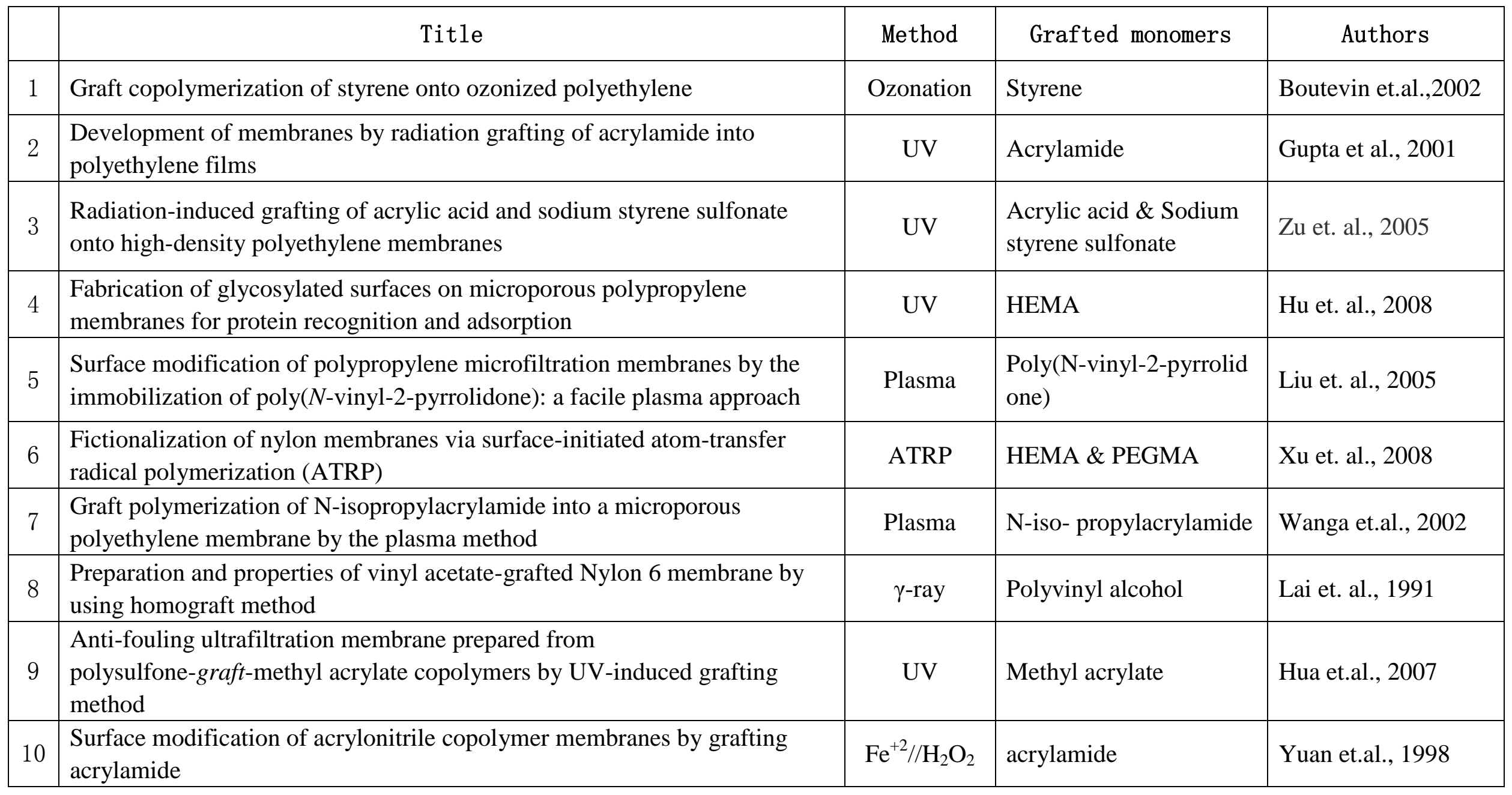




\section{Chapter 3 \\ Experimental Methodology}

\subsection{Materials}

\subsubsection{Selected polymer membranes and films}

- Commercial circular polypropylene membrane (PPMM) of $90 \mathrm{~mm}$ and $47 \mathrm{~mm}$ diameter with $0.22 \mu \mathrm{m}$ pore size (M02WP04700) and commercial polypropylene membrane sheet of $25.4 \mathrm{~cm} \times 3 \mathrm{~m}$ and $0.22 \mu \mathrm{m}$ pore size (M02WP00010) were purchased from GE Water \& Process Technologies (Oakville, Ontario, Canada).

-Biaxial oriented polypropylene (BOPP) film, $20 \mu \mathrm{m}$, T523-3 was purchased from AET film Co. Ltd.

The PPMMs and BOPPs are made from pure polypropylene polymers, which are highly hydrophobic without coating or co-extrusion layer. In this research, round polypropylene membrane pieces were used directly. The polypropylene membrane sheet was cut into $6.5 \mathrm{~cm} \times 10 \mathrm{~cm}$ pieces for ozonation, graft polymerization, and filtration test. BOPP film was cut into $3.81 \mathrm{~cm}(1.5 \mathrm{in}) \times 25.4 \mathrm{~cm}(10 \mathrm{in})$ strips for ozonation and graft polymerization.

\subsubsection{Other materials}

-Acrylic amide (AAm) 99.5\% was used for graft polymerization, and was purchased from VWR Canlab (Mississauga, Ontario, Canada). Acrylic amide in isopropanol alcohol (IPA 99.7\% ) solution was used for graft polymerization of monomers.

- 2-Hydroxyethyl methacrylate (HEMA) 99\% was used for graft polymerization, and was purchased from Sigma, Canada (produced by Alfa Aescar, a Johnson Matthhey Company).

- Polyethylene glycol 200 and Polyethylene glycol 600 (laboratory grade) were used for graft polymerization, and were purchased from Sigma, Canada (produced by Alfa Aescar, a Johnson Matthhey Company). Polyethylene glycol 3350 
(laboratory grade) was used for graft polymerization, and was purchased from Sigma, Canada.

- Albumin, bovine serum (BSA) $>98 \%$ was purchased from Sigma, Canada (produced by Calbiochem).

- Glacial acetic acid, solid potassium iodate, and solid sodium thiosulfate were used as titration reagents. All were laboratory grade and were obtained from Sigma-Aldrich (Toronto, Ontario).

- Other chemicals used were copper sulfate hydrate, ferric sulfate hydrate, sodium sulfate, ferrous sulfate hydrate, sodium phosphate monobasic monohydrate and sodium phosphate dibasic anhydrous, and nitrobenzene. They were all laboratory grade and supplied either by Sigma-Aldrich Canada (Oakville, Ontario, Canada) or VWR Canlab (Mississauga, Ontario, Canada).

\subsection{Experiment Setup}

\subsubsection{Experiment setup for membrane and film ozonation}

Figure 3.1 is a picture of the ozone generation and reaction system, and Figure 3.2 shows a schematic diagram of the ozonation reactor setup used in this research.

As shown in Figure 3.2, the oxygen cylinder provides oxygen to the ozone generator through the pressure control meter. The ozone is then generated and fed to the diffuser and reactor vessel through the adjustable valve and flow rate control meter. A three-way valve transports the ozone gas mixture to the analyzer, which measures the ozone concentration. The consumed ozone gas mixture is transported to the exhaust catalyst pipes, and decomposed ozone is released to the environment by the exhaust fan. 


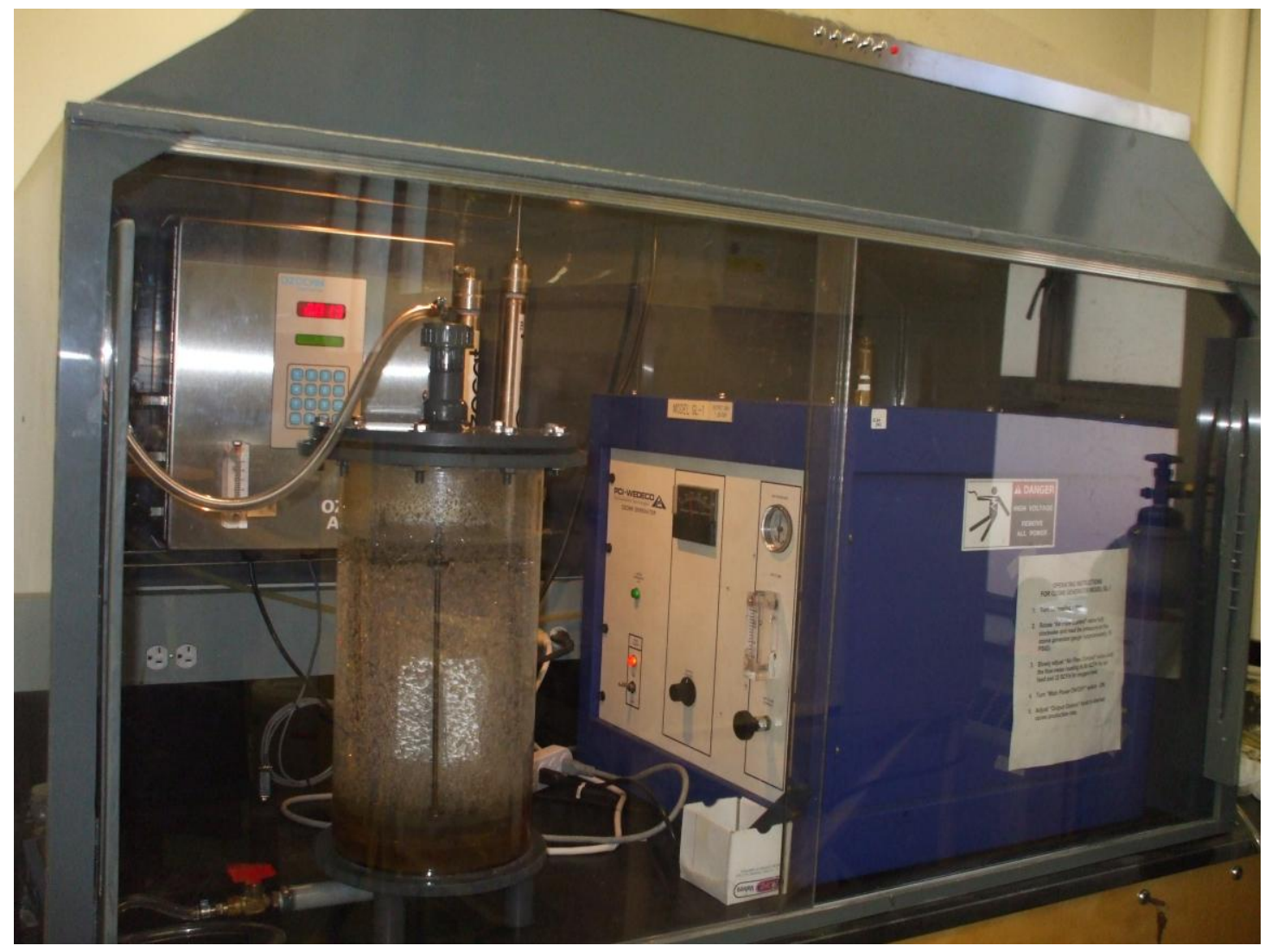

Fig. 3.1 The ozone generation and reaction system

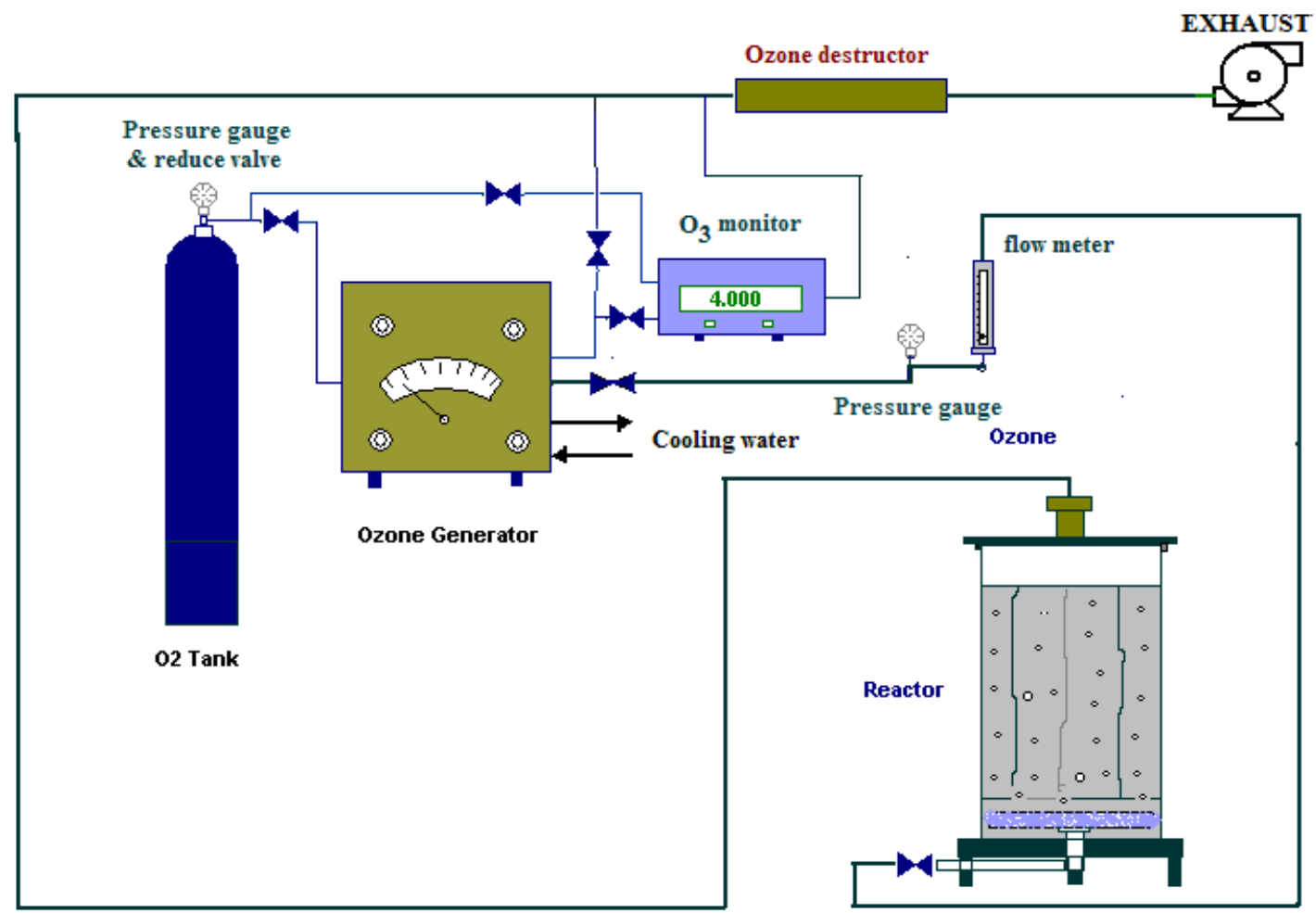

Fig 3.2 Schematic illustration of the ozone generation and reactor system 


\subsubsection{Experiment setup for graft polymerization}

Figure 3.3 shows the AAm graft polymerization incubator shaker, and Table 3.1 lists the equipment used in graft polymerization.

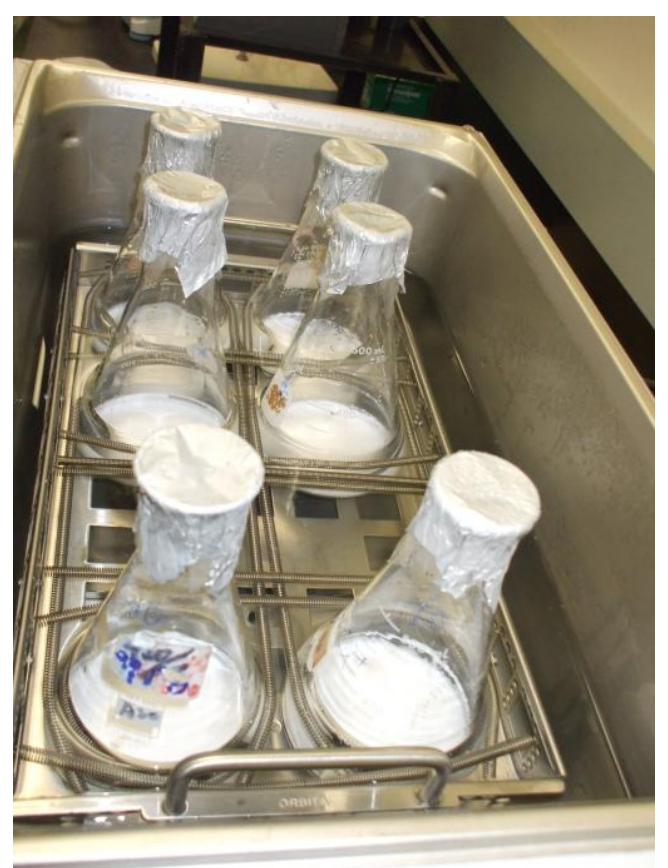

Fig 3.3 AAm grafting polymerization system

Table 3.1 Description of the equipment used in the graft polymerization reactor system

\begin{tabular}{|l|l|}
\hline Equipment & Description \\
\hline Nitrogen cylinder & from BOC Canada \\
\hline $\begin{array}{l}\text { Pressure meter and pressure } \\
\text { reducing valve }\end{array}$ & Specific for $\mathrm{N}_{2}$ application \\
\hline Shaking hot tub & $\begin{array}{l}\text { Boekel Scientific Products -290400, PA } \\
19053-9364, \text { USA }\end{array}$ \\
\hline Reactor & 250 and 500 ml Erlenmeyer flasks \\
\hline Exhauster & Connected to the O ${ }_{3}$ exhauster fan \\
\hline Ultrasonic cleaner & VWR International, Model 50D \\
\hline
\end{tabular}




\subsection{Experimental Procedure}

\subsubsection{The steps of the procedure}

The experiments were implemented in the following seven steps, and the conditions were shown in Table 3.2.

Step I: Ozonate polypropylene membranes and films at room temperature $\left(22-23{ }^{\circ} \mathrm{C}\right)$ in the aqueous phase, and analyze the peroxide generated and the contact angle changes.

Step II: Ozonate polypropylene membranes and films in the ozone gas mixture, and analyze the peroxide content and contact angle and compare to those of the aqueous phase's ozonation.

Step III: Based on the above experimental results, study the effect of the applied dose. Investigate the effects of $\mathrm{pH}$ at the selected applied ozone dose. Investigate the catalytic ozonation and mechanism.

Step IV: Based on the results of Step III, select suitable ozone dose, and conduct the graft polymerization of AAm.

Step V: Based on the results of Step III, select a suitable ozone dose, and conduct the graft polymerization of PEG.

Step VI: Based on the results of Step III, select a suitable ozone dose, and conduct the graft polymerization of HEMA.

Step VII: Employ adsorption test on the grafted and virgin PP films, and conduct filtration and fouling tests on the grafted membranes by using BSA solution. 
Table 3.2 Description of experimental steps of this research

\begin{tabular}{|c|c|c|c|c|c|c|c|}
\hline Step & $\begin{array}{l}\text { Reaction } \\
\text { medium }\end{array}$ & Substrate & $\begin{array}{c}\text { Applied } \\
\text { ozone } \\
\text { dose } \\
(w t \%)\end{array}$ & $\begin{array}{l}\text { Gas flow } \\
\text { rate for } \\
\text { ozonation } \\
\text { (L/min) }\end{array}$ & $\begin{array}{c}\text { Gas } \\
\text { pressure } \\
\text { for } \\
\text { ozonation } \\
\text { (psig) }\end{array}$ & $\begin{array}{l}\text { Reaction time } \\
\text { (hr) }\end{array}$ & $\begin{array}{c}\text { Reactor } \\
\text { volume } \\
\text { (L) }\end{array}$ \\
\hline Step I & $\begin{array}{l}\text { Aqueous } \\
\text { (DW) }\end{array}$ & $\begin{array}{l}\text { PPMM, } \\
\text { BOPP }\end{array}$ & 3.0 & 10 & 15 & $\begin{array}{l}15 \text { min to } 1.75 \\
\text { hrs ozonation }\end{array}$ & 10 \\
\hline Step II & Gaseous & $\begin{array}{l}\text { PPMM, } \\
\text { BOPP }\end{array}$ & 3.0 & 10 & 15 & $\begin{array}{l}15 \text { min to } 1.75 \\
\text { hrs ozonation }\end{array}$ & 10 \\
\hline Step III & $\begin{array}{l}\text { Aqueous } \\
\text { (DW) }\end{array}$ & PPMM & $\begin{array}{c}1.0 \text { to } \\
3.0\end{array}$ & 10 & 15 & $\begin{array}{l}15 \text { min to } 45 \\
\text { min ozonation }\end{array}$ & 10 \\
\hline Step IV & $\begin{array}{l}\text { AAm } \\
\text { solution }\end{array}$ & $\begin{array}{l}\text { PPMM, } \\
\text { BOPP }\end{array}$ & 3.0 & 10 & 15 & $\begin{array}{c}15 \& 45 \mathrm{~min} \\
\text { ozonation, } 20 \\
\text { hrs graft } \\
\text { polymerization }\end{array}$ & $\begin{array}{c}10 \\
250 \mathrm{ml}\end{array}$ \\
\hline Step V & $\begin{array}{l}\text { HEMA } \\
\text { solution }\end{array}$ & $\begin{array}{l}\text { PPMM, } \\
\text { BOPP }\end{array}$ & 1.0 & 10 & 15 & $\begin{array}{c}15 \text { min } \\
\text { ozonation, } 20 \\
\text { hrs graft } \\
\text { polymerization }\end{array}$ & $\begin{array}{c}10 \\
250 \mathrm{ml}\end{array}$ \\
\hline Step VI & $\begin{array}{c}\text { PEG } \\
\text { solution }\end{array}$ & PPMM & 1.0 & 10 & 15 & $\begin{array}{c}15 \text { min } \\
\text { ozonation, } 20 \\
\text { hrs graft } \\
\text { polymerization }\end{array}$ & $\begin{array}{c}10 \\
250 \mathrm{ml}\end{array}$ \\
\hline $\begin{array}{l}\text { Step } \\
\text { VII }\end{array}$ & $\begin{array}{l}\text { Filtration } \\
\text { test, } \\
\text { protein } \\
\text { adsorption }\end{array}$ & $\begin{array}{l}\text { PPMM } \\
\text { BOPP }\end{array}$ & 1.0 & 10 & 15 & $\begin{array}{l}15 \text { min to } 1.0 \\
\text { hrs ozonation, } \\
\text { and graft } \\
\text { polymerized } \\
\text { after that. }\end{array}$ & $\begin{array}{c}250 \mathrm{~mL}, 50 \\
0 \mathrm{~mL}\end{array}$ \\
\hline $\begin{array}{l}\text { Remark } \\
\text { : }\end{array}$ & \multicolumn{7}{|c|}{$\begin{array}{l}\text { All the reactions except graft polymerization were carried out at room temperature. } \\
\text { DW indicates distilled water }\end{array}$} \\
\hline
\end{tabular}

\subsubsection{The ozonation procedure}

- Compressed oxygen gas was fed to the ozone generator (PCI-WEDECO Environmental Technologies, Model GL-1); flow was controlled by the flow meter and pressure gauge;

- Cooling water was used to remove the heat from the generator; 
- Ozone was generated at a controlled rate to ensure that it was a certain percentage of the oxygen gas mixture (rate was controlled by adjusting the output power knob) in the generator and was released in three ways: to the reactor (flow rate and pressure were measured and controlled), to the exhaust catalyst pipes to be released, and then to the ozone gas analyzer (PCI-WEDECO Environmental Technologies OZONE MONITOR);

- Controlled ozone and oxygen gas mixture was fed to the reactor through the diffuser (ceramic, $2-5-\mu$ porous hole size, 7.5 -inch diameter), through which it was uniformly diffused in the aqueous phase or gas phase in the reactor.

- Pretreated, precisely cut, specific samples of PPMM/BOPP were fixed in the reactor prior to the start of the reaction. Samples reacted with ozone at set concentrations and set reaction times, as shown in Table 3.2.

- Samples were hung on the frame inside the reactor, and selected polymer membranes or films were ozonated at room temperature $\left(22-23{ }^{\circ} \mathrm{C}\right)$ with ozone gas mixture in the aqueous (reactor filled with distilled water) or gaseous phase to gain enough peroxides and maintain mechanical strength;

- When the target ozonation time finished, turn off the $\mathrm{O}_{3}$ generator, and lead in the $\mathrm{O}_{2}$ to purge the reaction system until the ozone detector shows the ozone concentration to 0 .

- Samples were taken out when the reaction was finished. Samples were then vacuum dried at room temperature;

- Used the standard iodide metric method to analyze the generated peroxide concentration; used the Fourtier Transform Infrared (FTIR) to test the functional groups; used Gonoimeter to test the contact angle of the ozonated membranes/films;

- Investigated the effects of the applied dose and reaction time. Ozone concentration in ozone and oxygen gas mixture can be from $0.1 \mathrm{wt} \%$ to $3.5 \mathrm{wt} \%$; ozonation time can be 15 minutes to 1.5 hours, depending on the peroxide concentration needed and the remaining mechanical strength of the substrates;

- Added catalysts such as $\mathrm{Cu}^{2+}$ and $\mathrm{Fe}^{3+}$ in the aqueous phase, and investigated the ozonation under catalytic conditions; 


\subsubsection{The graft procedure}

Dried samples were used for graft polymerization to investigate the radical reaction of graft polymerization after ozonation. The equipment was as shown in Table 3.1.

- Graft polymerization was performed after ozonation by soaking the ozone pretreated membranes under specific conditions (different temperature and reaction time) in different monomer solutions, which had been purged of oxygen by $\mathrm{N}_{2}$ gas. Different graft polymerizations were studied and compared;

- Monomers used were polyethylene glycol (PEG), Acrylamide (AAm), 2-Hydroxyethyl Methacrylate (HEMA). These monomers/oligomers, which have hydrophilic functional groups, can improve the hydrophilicity when grafted to the membranes.

- Graft polymerization was stopped by adding cold distilled water to the reactors; samples were put through the washing procedure;

- Washed grafted samples were dried under vacuum and kept at room temperature;

- The changes in hydrophilicity of the modified membranes were tested by the contact angle.

- The functional groups grafted onto the membranes were tested by the FTIR instrument;

- The changes in the amorphousity of the surface and pores were checked by the Scanning Electron Microscope (SEM).

\subsubsection{The filtration test and protein adsorption procedure}

- Dried BOPP samples were employed in the BSA protein adsorption test. The method was modified by the author of this thesis from Hu et al. (2008) and Wang et al. (1999). Protein solutions comprised $2000 \mathrm{mg} / \mathrm{L}$ BSA in a phosphate-buffer solution (pH 6.9). After the samples were soaked in BSA solution for 16 hours at $50^{\circ} \mathrm{C}$, samples were dried under vacuum and sent to do the SEM test;

- Dried PP membrane samples were set up in the filtration test device after being pre-wetted; 
- Filtration and fouling tests were conducted by using BSA protein solution in the dead-end membrane filtration device (easy to fix the membrane and get filtration data).

- The flux of BSA solution permeating through the virgin and modified membranes was tested, and the fouling differences were compared;

(i) The flux testing system is shown in Figure 3.4. It comprises a water/solution tank, pumps, membrane holder, permeate flow line, reject flow line, and return flow loop. This device is designed for testing microfiltration membranes. Applied pressure is $1-50 \mathrm{psig}$, and flux of permeate is $0.1-3.0 \mathrm{~L} / \mathrm{min}$.

(ii) The process was conducted according the design of the author, who referred to the report of Chen and Belfort (1999). The trans-membrane pressure was kept constant at 3-10 psig, and the reverse DW washing pressure was 20 psig for the dead-end device. The permeation data were collected at fixed time intervals and the permeate solution was automatically weighed to determine the trans-membrane flux. Protein solutions comprised $1000 \mathrm{mg} / \mathrm{L}$ BSA in a phosphate-buffer solution ( $\mathrm{pH}$ 6.9).

- After the filtration test, the membrane samples were cut in half. One half of the sample was washed on surface using DW, and the other half kept in original fouling condition. Both were dried under vacuum, and then sent to do SEM test.

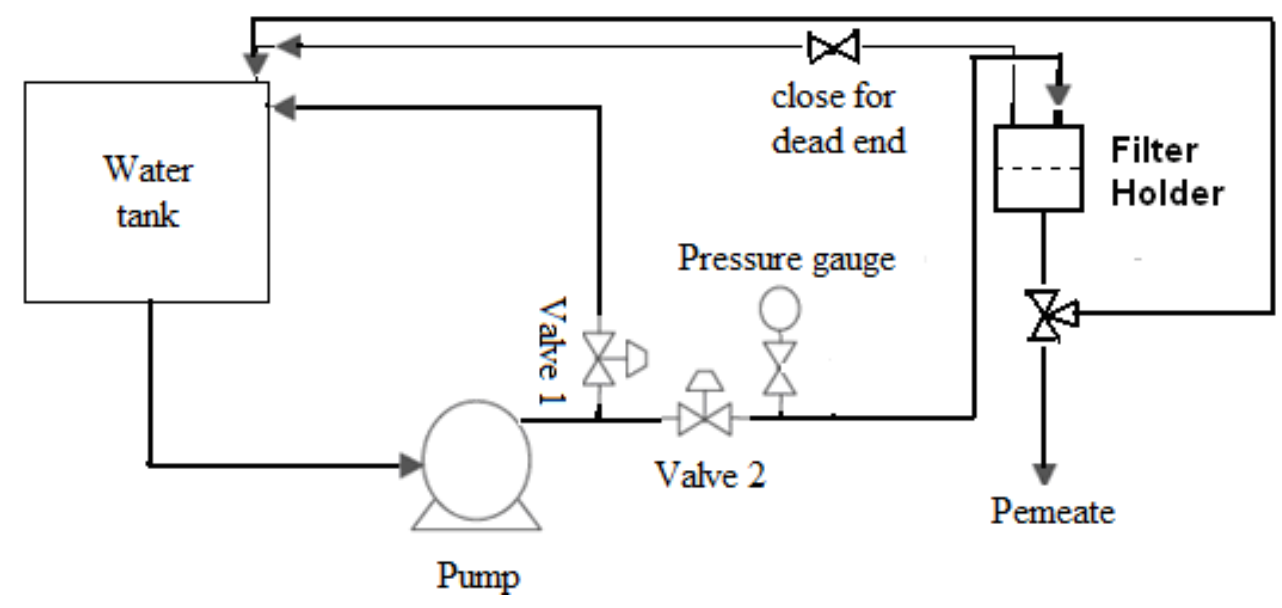

Fig 3.4 Schematic illustration of membrane flux test system 


\subsection{Experimental Implementation}

\subsubsection{Ozonation without catalysis}

Ozonation of PP substrates, in distilled water and in gaseous phase, and in different doses, different $\mathrm{pH}$ value, with or without scavengers, and the ozonation aging test, are designed in the following sections.

\subsubsection{Ozonation in aqueous phase}

Pretreatment: Used the round PP membranes directly; the BOPP strips were cut from films and washed with distilled water (soaked in distilled water for half an hour, and then dried at room temperature).

$10 \mathrm{~L}$ distilled water was placed in the reactor. Pretreated samples were fixed to the stainless steel frame to prevent them from sticking to or overlapping one another, and then put into the aqueous reactor; polypropylene membranes and films reacted with ozone according to the ozonation procedure.

After ozonation, the samples were taken out according to the desired reaction times: $15 \mathrm{~min}, 30 \mathrm{~min}, 45 \mathrm{~min}, 60 \mathrm{~min}, 75 \mathrm{~min}, 90 \mathrm{~min}$, and $105 \mathrm{~min}$. The water was removed with clear tissue paper and samples were vacuum dried at room temperature $\left(22-23{ }^{\circ} \mathrm{C}\right)$ for 1.5 to 2 hours. Dried samples were sent for analysis and further treatment.

The ozone doses applied were all set at $3.0 \mathrm{wt} \%$ in both aqueous and gaseous phase ozonation, according to the results of our preliminary tests.

\subsubsection{Ozonation in gaseous phase}

For the gaseous phase ozonation, the procedure was analogous to the aqueous phase's ozonation, except that there was no water inside the reactor.

\subsubsection{Investigation of the ozone dose}

Ozone dose of $1.0 \mathrm{wt} \%, 2.0 \mathrm{wt} \%$, and $3.0 \mathrm{wt} \%$ in oxygen mixture was applied to PPMM ozonation, respectively. The generated peroxide concentrations were analyzed, and the samples reacted with ozone in the aqueous and gaseous phases at the same $\mathrm{O}_{3}$ applied 
dose and for the same treatment time were compared. It was determined which ozone dose and methods would be used for subsequent graft polymerization.

The investigation of the reaction conditions was as follows:

1. Both in the aqueous phase and the gaseous phase, treatment time was set at 0.5 hours, and different applied ozone doses, from $1.0 \mathrm{wt} \%$ to $3.0 \mathrm{wt} \%$ were studied to determine which dose would result in the highest peroxide content. The results were compared.

2. The $\mathrm{O}_{3}$ concentration was set at $3.0 \% \mathrm{wt}$, and different reaction times, from $15 \mathrm{~min}$ to $1.75 \mathrm{~h}$, were used to determine which reaction time yielded the highest peroxide content (and maintained the highest mechanical strength of the BOPP films).

\subsubsection{The study of the effects of varying $\mathrm{pH}$ value in aqueous phase reactions}

In aqueous phase reactions, $\mathrm{O}_{3}$ concentration was set at $3.0 \% \mathrm{wt}$ and treatment time at $0.5 \mathrm{~h}$, and $\mathrm{pH}$ value was varied from 3.0 to 10.0 , which was adjusted by using $1.0 \mathrm{M} \mathrm{H}_{2} \mathrm{SO}_{4}$ and $1.0 \mathrm{M} \mathrm{NaOH}$, and the results were studied.

\subsubsection{Scavenger study}

In the aqueous phase, both molecular ozone and free radicals were present. In order to make clear whether both contributed to the generation of peroxide and which one made the main contribution, a radical scavenger test was conducted to find the answers to these questions. Radical scavengers are chemicals which consume the radicals generated during the reaction. The scavenger sodium carbonate was added to the distilled water to eliminate the free radicals.

\subsubsection{Washing test}

A washing test was done in order to identify the peroxide content and graft polymerization difference between the gaseous phase ozonation and the aqueous phase ozonation. 10\% (v/v) IPA (without IPA, the PPMM surface will hardly get wet in DW) in distilled water was employed to wash the gaseous ozonated PPMM and the aqueous ozonated PPMM. After 15 minutes respectively, the peroxide concentration was analyzed and 
compared to the non-washed ozonated PPMM. This test also was conducted on the BOPP films.

\subsubsection{Aging study}

To study the stability of the peroxide content generated on the films, the aging test was performed for BOPP treated with ozone in the aqueous phase. For each experimental run, the reaction time was one hour and the applied ozone dose was controlled at $3.0 \mathrm{wt} \%$. The films were examined for their peroxide concentration immediately after ozonation, after 3 days, after two weeks, and after 4 weeks.

\subsubsection{Catalytic ozonation}

\subsubsection{1 $\mathrm{Fe}^{3+}$ ion used as the catalyst}

$\mathrm{FeCl}_{3} \cdot 6 \mathrm{H}_{2} \mathrm{O}$ was weighed precisely and added to $10 \mathrm{~L} \mathrm{DW}$ in the reactor and stirred till it dissolved completely; the $\mathrm{pH}$ value was then measured; $\mathrm{O}_{3}$ gas was added to the reactor for 1 minute of pretreatment; the samples were placed in the reactor, and the ozonation was started. The concentration of $\mathrm{Fe}^{3+}$ ions was set at different amounts $(\mathrm{g} / \mathrm{L})$ for different experiments, and the ozonation time was fixed at 30 minutes. The amount of peroxide generated was measured and compared.

\subsubsection{2 $\mathrm{Cu}^{2+}$ ion used as the catalyst}

$\mathrm{CuSO}_{4} \cdot 5 \mathrm{H}_{2} \mathrm{O}$ was weighed precisely and added to $10 \mathrm{~L} \mathrm{DW}$ in the reactor and stirred till it dissolved completely; the $\mathrm{pH}$ value was then measured; $\mathrm{O}_{3}$ gas was added to the reactor for 1 minute of pretreatment; the samples were placed in the reactor and the ozonation was started. The concentration of $\mathrm{Cu}^{2+}$ ions was set at different amounts $(\mathrm{g} / \mathrm{L})$ for the different experiments and the ozonation time was fixed at 30 minutes; the amount of peroxide generated was measured and compared; the concentration of $\mathrm{Cu}^{2+}$ ions was set at $0.1 \mathrm{~g} / \mathrm{L}$ $(0.7868 \mathrm{mmol} / \mathrm{L})$ and ozonation time was set at from 15 minutes to 60 minutes; the amount of peroxide generated was measured and compared. 


\subsubsection{Investigative tests of the mechanism on catalyst $\mathrm{Cu}^{2+}$}

\subsubsection{1 $\mathrm{Cu}^{2+}$ catalytic ozonation applied at a low temperature}

A low temperature $\mathrm{Cu}^{2+}$ catalytic ozonation was conducted for 15- and 30-minute periods, respectively. The PPMM was ozonated using the same $\mathrm{O}_{3}$ dose as in Section 3.4.2.2; the temperature was set at $0{ }^{\circ} \mathrm{C}$ instead of room temperature. The generated peroxide was tested.

\subsubsection{Scavenger tests in $\mathrm{Cu}^{2+}$ catalytic ozonation}

Scavengers of $3.0 \mathrm{~g} / \mathrm{L} \mathrm{Na}_{2} \mathrm{CO}_{3}$ or $0.1 \mathrm{~mol} / \mathrm{L} \mathrm{t}$-butanol was added to the $\mathrm{Cu}^{2+}$ catalytic ozonation $(1.574 \mathrm{mmol} / \mathrm{L})$, respectively, $3.0 \mathrm{wt} \%$ ozone concentration and ozonation time from 15 minutes to 45 minutes were set up and tested. Similar tests were done at $0^{\circ} \mathrm{C}$.

\subsubsection{The effect of the anion $\mathrm{SO}_{4}{ }^{2-}$ on $\mathrm{DW}$ ozonation}

The effect of the anion $\mathrm{SO}_{4}{ }^{2-}$ was investigated in aqueous phase ozonation. Concentration of $\mathrm{SO}_{4}{ }^{2-}\left(\mathrm{Na}_{2} \mathrm{SO}_{4}\right.$ applied as a provider of $\left.\mathrm{SO}_{4}{ }^{2-}\right)$ was from $5 \times 10^{-4} \mathrm{~mol} / \mathrm{L}$ to 5 $\mathrm{x} 10^{-2} \mathrm{~mol} / \mathrm{L}$, which simulated the applied $\mathrm{SO}_{4}{ }^{2-}$ concentration range in ozonation with the catalyst $\mathrm{CuSO}_{4} \cdot 5 \mathrm{H}_{2} \mathrm{O}$.

\subsubsection{Investigation of dissolved ozone in DW undergoing $C u^{2+}$ catalytic ozonation}

In order to investigate the dissolved ozone concentration, an indigo test of potassium indigo trisulfonate was employed. The dissolved ozone concentration was tested in the copper catalytic ozonation both at room temperature and at $0{ }^{\circ} \mathrm{C}$.

3.4.3.5 Hydroxyl radical capture by Nitrobenzene (NB) applied to ozonation with or without $\mathrm{Cu}^{2+}$ catalyst

A method of special radical capture is applied to demonstrate which radical was generated in the catalytic ozonation. Nitrobenzene is a good chemical for capturing the hydroxyl radicals. The author referred to Feng et al. (2011) "Experimental Determination of Hydroxyl Radical Reactivity in Supercritical Water Using Pulse Radiolysis,” and applied 25 $\mathrm{ml} \mathrm{NB}$ (99\% purity) to $75 \mathrm{ml}$ ozonation solution at different ozonation times to capture the 
hydroxyl radicals by forcefully shaking the flasks for 5 minutes. NB and water were separated after the capture procedure, and the samples of reacted NB were removed and tested using UV-Vis at the single wavelength of $410 \mathrm{~nm}$.

\subsubsection{Graft polymerization of the specific film under selected conditions}

\subsubsection{AAm graft polymerization of the specific film}

After the ozone dose tests, the ozonation concentration for graft polymerization was chosen at $3.0 \mathrm{wt} \%$, and the ozonation time was 15 minutes. After ozonation and degassing, the PP membranes were immersed in $50 \mathrm{ml} 20 \%$ AAm in IPA. The solutions containing the membranes were degassed again by nitrogen purge. Thereafter, they were heated to $80{ }^{\circ} \mathrm{C}$ for 20 hours in an incubator shaker at a speed of $120 \mathrm{rpm}$. After adding cold distilled water to the flask, the PPMM in the flasks underwent the following steps: a) the PPMM was immersed in cold distilled water in the flask for 1.5 hours with the distilled water being changed twice, $b$ ) the flasks with PPMM and changed DW were placed inside in $60{ }^{\circ} \mathrm{C}$ incubator shaker for half an hour at a shaking speed $80 \mathrm{rpm}, \mathrm{c}$ ) the DW was poured out and the flasks were filled with acetone and then put in a $50{ }^{\circ} \mathrm{C}$ incubator shaker at a speed of $50 \mathrm{rpm}$ for half an hour, d) the acetone was removed and distilled water was added and the flasks were kept at room temperature for 8 hours, and e) PPMM samples were dried at room temperature under vacuum. The author referred to Tu et al. (2005) for the reaction and cleaning methods, but these were modified by author.

Dried samples were taken to examination by means of FTIR contact angle and scanning electronic microscope.

\subsubsection{PEG graft polymerization of the specific film}

The ozonation dose was chosen at $3 \mathrm{wt} \%$ and the ozonation time was 15 minutes. After ozonation and degassing, the PPMM membranes were immersed in $50 \mathrm{ml} 20 \%$ PEG solutions; PEG200, PEG600, and PEG 3350 were used for the different experiments. The solutions containing the membranes were degassed again by nitrogen purge, then heated to $60{ }^{\circ} \mathrm{C}$ for 20 hours in an incubator shaker at a speed of $120 \mathrm{rpm}$. After adding cold distilled water to the flasks, the PPMM membranes in the flasks underwent the cleaning procedure. 
Dried samples were taken to examine by means of FTIR, contact angle, and scanning electronic microscope.

\subsubsection{HEMA graft polymerization of specific film}

The ozonation dose was chosen at $3 \mathrm{wt} \%$ and the ozonation time was 15 minutes. After ozonation and degassing, the PPMM membranes were immersed in flasks containing $50 \mathrm{ml} \mathrm{20 \%} \mathrm{HEMA} \mathrm{solutions.} \mathrm{The} \mathrm{solutions} \mathrm{containing} \mathrm{the} \mathrm{membranes} \mathrm{were} \mathrm{degassed} \mathrm{again}$ by nitrogen purge, then they were heated to $55{ }^{\circ} \mathrm{C}$ for $20 \mathrm{~h}$ in an incubator shaker at a speed of $120 \mathrm{rpm}$. After adding cold distilled water to the flasks, the PPMM membranes underwent the cleaning procedure. Dried samples were taken to examine by means of FTIR, contact angle, and scanning electronic microscope.

\subsubsection{Dead-end filtration test}

The membranes were examined for anti-fouling properties before and after surface modification. The filtration flow rate and fouling tests were conducted by a filtration setup as shown in Fig 2.4a. With a bypass loop, the pressure and flow rate can be adjusted. The container and pipe loop were designed by the author and built by the Chemical Engineering Department workshop of Ryerson University. The membrane holder (Canlab stainless steel pressure filter holder, $47 \mathrm{~mm}, 200 \mathrm{ml}$ ) and pumps were purchased. 1000mg/L BSA solution (buffered by phosphate at $\mathrm{pH}$ 6.9) was employed in the filtration test. Pressure was set at 10 psig for each filtration. The modified and virgin membranes were put through filtration till the permeate weight dropped down to less than 2 g per 5 seconds; then the fouled membranes were reversed and $600 \mathrm{ml} \mathrm{DW}$ water was used to back flush the fouled membranes. The back flush pressure was set at 20 psig. Next, the back flushed membrane was reversed, and a second filtration test was conducted using the fresh BSA solution. The weight of the filtration

permeate was recorded every 5 seconds by an Ohaus AV2101 auto balance (Ohaus Corporation, NJ, USA), which was connected to a computer recording system. The recorded data were transferred to Excel and underwent calculations, and charts of the flow rate versus time were made to compare the BSA filtration of the virgin and modified PPMM. 


\subsection{Analytical Methods}

The following analytical methods were used in this research:

\subsubsection{Standard metric iodide method}

To analyze peroxide, solutions were prepared using the ratio of $50 \mathrm{ml}$ isopropanol : 2 $\mathrm{ml}$ glacial acetic acid $+2 \mathrm{ml}$ saturated KI. $50 \mathrm{ml}$ of solution was added to the samples (pieces of $0.22 \mu \times 47 \mathrm{~mm}$ round membranes or pieces of films with the dimensions 1.5 in $\times 10 \mathrm{in}$ ). The mixture was heated almost to boiling $\left(83-84{ }^{\circ} \mathrm{C}\right)$ for 5-7 $\mathrm{min}$, and then titrated with $0.001 \mathrm{~N}$ standard sodium thiosulfate solution until the yellow color disappeared. The consumption of $0.001 \mathrm{~N}$ standard sodium thiosulfate was recorded, and the peroxide content was calculated (Tu et al., 2006; Yuan et al., 2003).

The principal of this test is that peroxide/hydroperoxide reacts with sodium iodide to produce iodine in an acidic environment, and iodine can be precisely titrated with standard sodium thiosulfate solution. Detailed calculations can be found in Appendix I.

\subsubsection{FTIR method}

FTIR (Perkin Elmer Spectrum One, V3.01 instrument) was used to examine functional groups on the surface of polymer films (Fujimoto et al., 1993; Wang et al., 2000). The wavelength scanned was $450 \mathrm{~nm}-4000 \mathrm{~nm}$.

- For Polyolefin, the peaks for $\mathrm{C}=\mathrm{O}$ are at 1710 to $1750 \mathrm{~cm}^{-1}$; and the peaks for $\mathrm{C}-\mathrm{O}-\mathrm{C}$ are at 1080 to $1110 \mathrm{~cm}^{-1}$.

- The peak at $1668 \mathrm{~cm}^{-1}$ is a characteristic frequency of the $\mathrm{C}-\mathrm{N}$ amide group, and the peak at $3347 \mathrm{~cm}^{-1}$ is the characteristic frequency of $\mathrm{N}-\mathrm{H}$.

- C-H stretching and vibration are from 2850 to $3300 \mathrm{~cm}^{-1}$.

- The O-H peak is at $3290 \mathrm{~cm}^{-1}$ [carboxyl] and $3550 \mathrm{~cm}^{-1}$ [hydroxyl].

- Other functional group peak values are shown in Appendix V.

(Davidson et al., 2004; Mitchell et al., 2005; Wu et al., 2000; Yong et al., 2005; Yuan et al., 2002) 


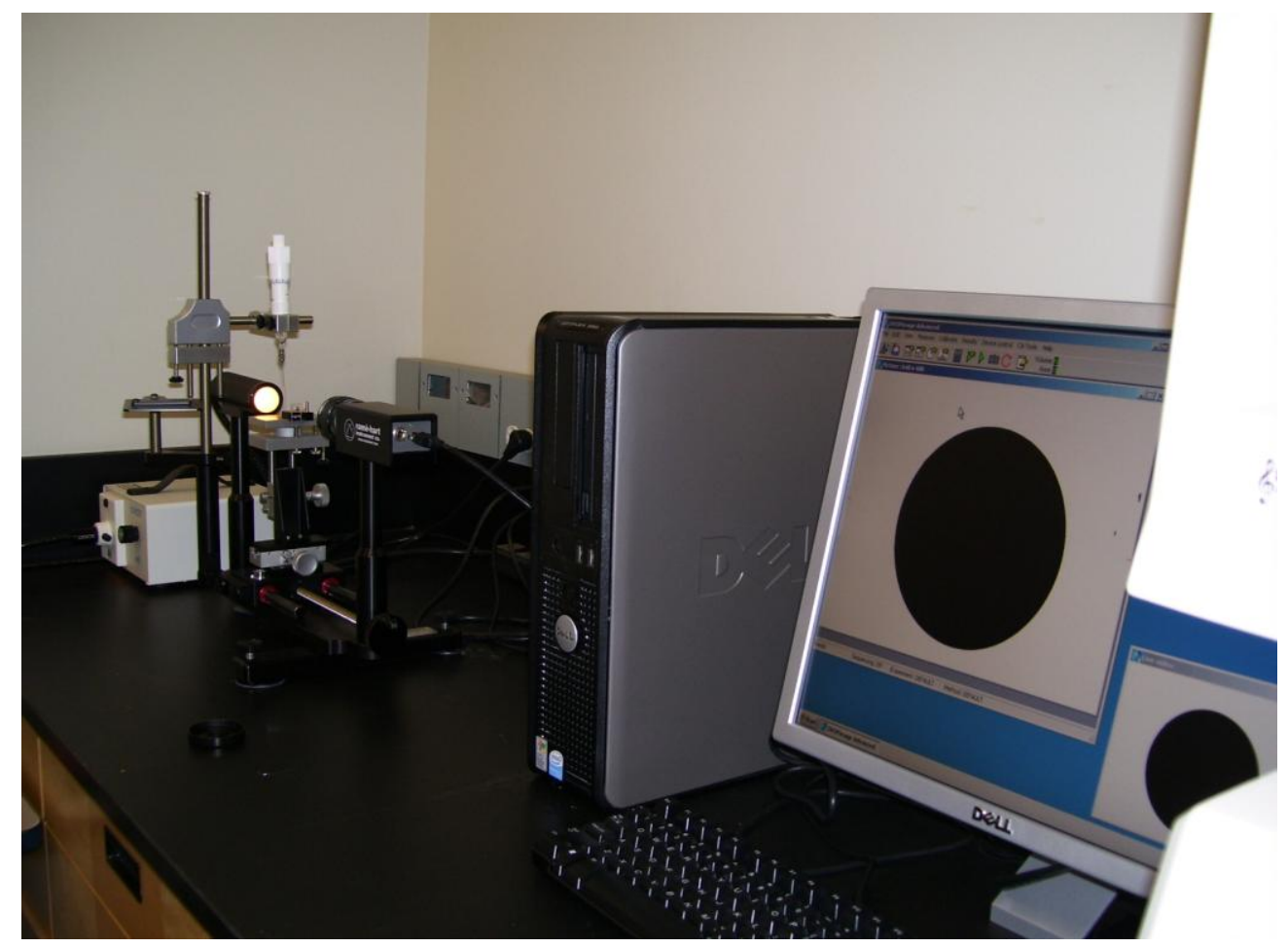

Fig 3.5 Goniometer photo -1

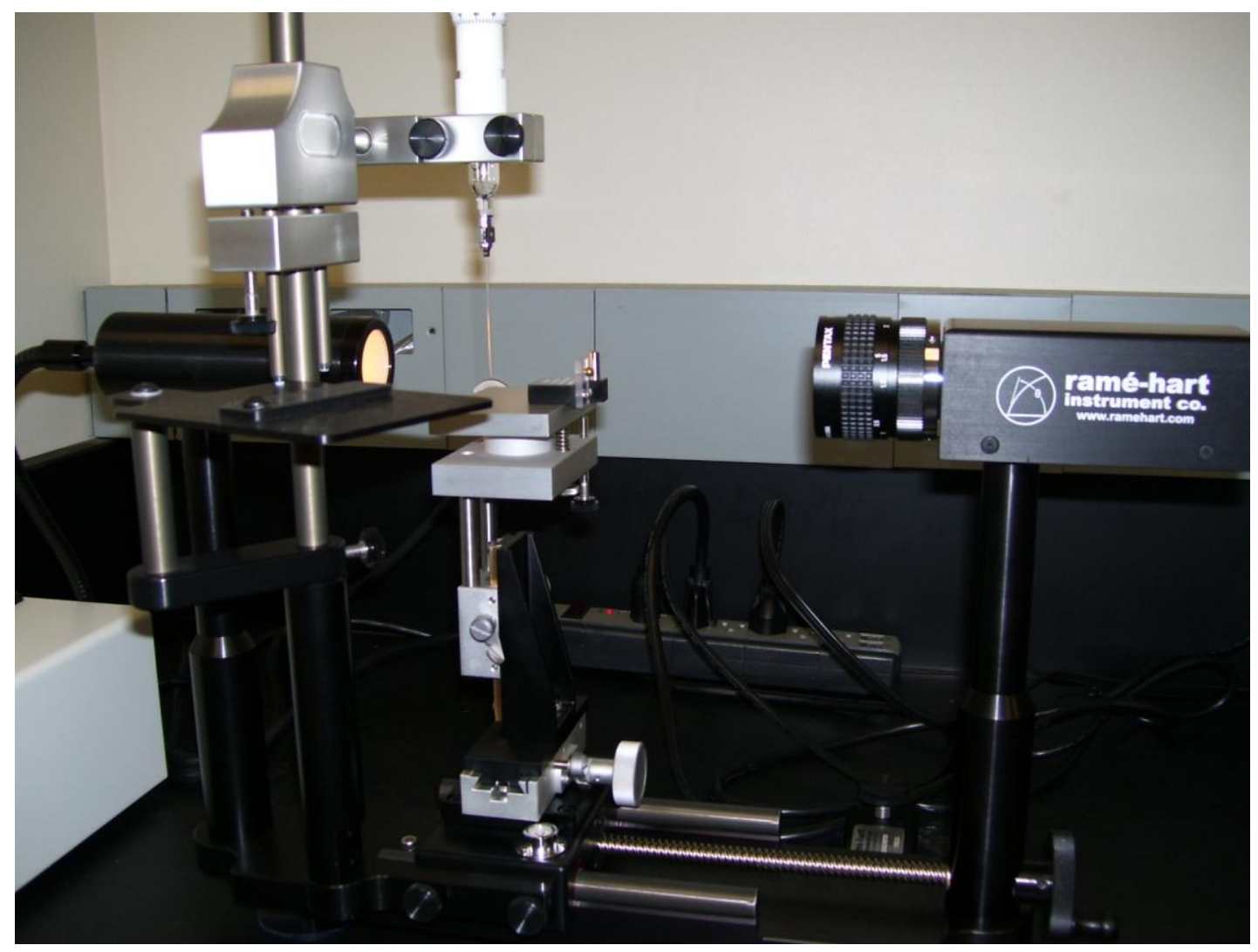

Fig 3.6 Goniometer photo-2 


\subsubsection{Contact angle method}

The contact angle of the film surface was measured to quantify the change in its hydrophilicity. The film samples were placed on a goniometer (Model 100-00-115, Rame Hart, NJ, USA), a sessile drop of super-pure water was placed on the film sample, and then the static angle was measured (Fujimoto et al., 1993; Yong et al., 2005). Figure 3.5 and Figure 3.6 are photos of the goniometer.

\subsubsection{SEM method}

Scanning Electron Microscope (SEM) images of the virgin, oxidized, and grafted film samples were taken with a JSM-35 microscope (JEOL, Japan) at 15-20 kV. Samples were pre-coated with gold at 0.2 Torr before the SEM analysis (Gatenholm et al., 1996). Samples were observed under 30 to 5000 times magnification.

\subsubsection{X-ray diffraction (XRD) method}

X-ray diffraction (XRD) of the membranes was used to test the crystallinity, and measured by a Rigaku Geigerflex machine (Rigaku MSC, TX, USA) with the tube voltage at $40 \mathrm{kV}$ and the tube current at $45 \mathrm{~mA}$.

As amorphous areas are damaged by ozone treatment, rearrangement of associated chains can occur, leading to an increase in the crystallinity/amorphousity ratios (Tu et al., 2006). Also, as exposure ensues, the polymer chains may decrease in length and their mobility increases, once again encouraging the formation of more ordered crystalline regions. Generally, the increase in crystal structure is the main factor in the stiffer and more brittle character of these materials (Kokatnur et al., 1941); and the reverse holds true for the increase in amorphousity. These results should be taken into consideration in studying the mechanical properties of the treated membranes and films (Michael et al., 2004).

\subsubsection{UV-Vis spectrometry method}

The ozone solution and catalytic ozonation solution were tested in a $1 \mathrm{~cm}^{3}$ cuvette placed in the UV-Vis spectrometer (Ultraspec 50, Bichrom Ltd., Cambridge, England). The testing wavelength was fixed at a particular $\mathrm{nm}$ or scan from $200 \mathrm{~nm}$ to $750 \mathrm{~nm}$. The 
absorption index, or chart, was recorded. The UV-Vis method is used to test the concentration of ozone or nitrobenzene in solution.

\subsubsection{Indigo method}

The method is to pipet $3.0 \mathrm{ml}$ of prepared indigo reagent II into a $10 \mathrm{ml}$ volumetric flask and dilute to $10 \mathrm{ml}$. Prepare a volumetric flask for each sample and the blank. Pipet 1.0 ml ozone solution into the flask and shake immediately; determine the absorbance of the blank and the samples at $600 \mathrm{~nm}$ UV using a 1-cm cuvette (Markee et al., 2009; Appendix IV). This method is used to test $\mathrm{O}_{3}$ molecular concentration in water. 


\section{Chapter 4}

\section{Results and Discussion}

The most important results presented in this work were obtained from ozone treatment of selected membranes and films in an aqueous medium. The results from ozone gas mixture treatment were obtained for comparison. PPMM and BOPP were used as the substrates to investigate the effects of operating parameters, graft polymerization on surface hydrophilicity enhancement and filtration tests.

Through oxidization by ozone of membrane/film surfaces, peroxide groups were generated on the surfaces. Those peroxides provided active species which initiate graft polymerization with vinyl functional group monomers. Consequently, hydroxyl, carboxyl, or amine groups were brought to the surfaces of the polymers. Thereafter, hydrophilicity was improved. Membranes with improved hydrophilicity can increase the anti-fouling of organic and protein materials.

Generally, a decrease in tensile strength results from chain scission, and cross-linking reactions as a result of ozone exposure that causes molecular chains to be stiffened. Also, the increase in crystal structure is the factor that causes the stiffer, more brittle character of the films.

Different monomers were applied to ozonated PP membranes and films. The grafting polymerizations were studied. The analysis that followed revealed the changes. The modified membranes were used for filtration, and the improvement in anti-fouling ability was determined after the modifications.

\subsection{Polypropylene Membrane (PPMM) Ozonation}

Polypropylene microfiltration membranes are highly hydrophobic, and the contact angle of PPMM is $120-130^{\circ}$. This hydrophobic property leads to fouling caused by the easy absorption of organic materials, thus PPMM is extremely limited in its applications. The following experiments focused on aqueous ozonation and graft polymerization to effectively improve the hydrophilicity and anti-fouling properties. 


\subsubsection{Ozonation of PPMM in aqueous and gaseous phases}

In this part of the experiment, PP membranes were ozonated in distilled water and in a gaseous mixture, respectively, to compare the efficiency of ozonation in different reaction media. Ozonation was conducted at $3.0 \mathrm{wt} \%, 10 \mathrm{~L} / \mathrm{min}$ gas flow rate, pressure of $103.4 \mathrm{kPa}$ (15 psig), and temperature of $22-23^{\circ} \mathrm{C}$. Figure 4.1 shows the results. The peroxide generation increased with ozonation time regardless of whether the ozonation was conducted in the aqueous or the gaseous phase. When the same ozone dose was applied, the detected peroxide concentration of the aqueous phase ozonation was a little lower than that of the gaseous phase ozonation. The relationship of peroxide generation with ozonation time in aqueous phase or in gaseous phase will be discussed in the Section 4.6.

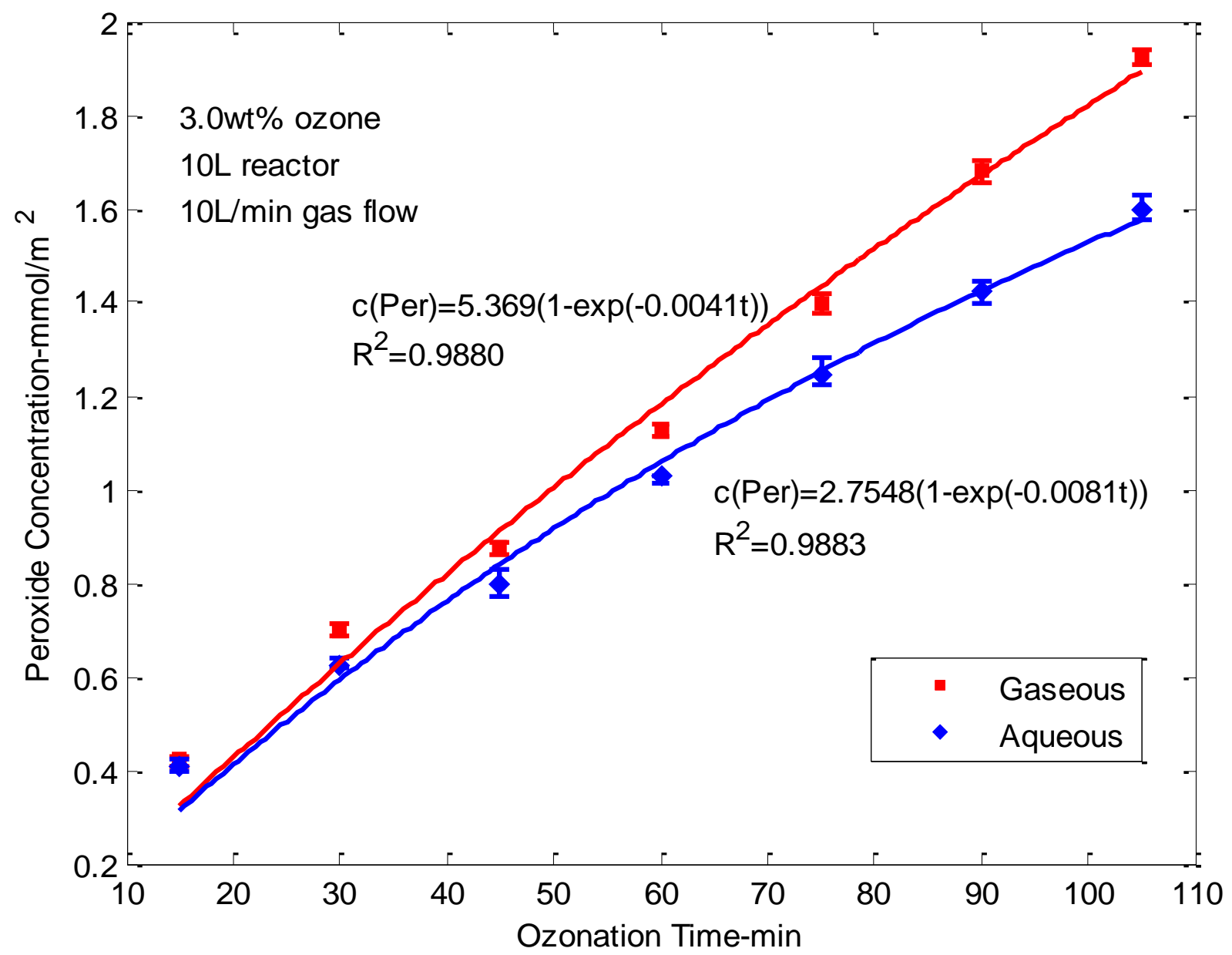

Fig 4.1 Peroxide concentration after ozonation of PPMM in the aqueous and gaseous phases. Peroxide $\left(\mathrm{mmol} / \mathrm{m}^{2}\right)$; Ozonation $3.0 \mathrm{wt} \%$ (time in minutes). 


\subsubsection{Washing test}

To explore why aqueous ozonation produced less peroxides, the ozonated membranes were washed to find changes in peroxide concentration. As shown in Figure 4.2, the PPMM membrane that was ozonated in the gaseous phase was washed immediately after degassing using distilled water plus Isopropyl Alcohol (IPA) (9:1 in volume, 10\% IPA mixed in, to increase the wettability) and compared to the non-washed PPMM membrane that was ozonated in the gaseous phase as well. It was found that PPMM had a much lower peroxide concentration after washing than the non-washed PPMM. The hypothesis used to explain this phenomenon is that when ozone came into contact with the PPMM, peroxide was generated on the backbone molecules. At the same time, chain scission occurred in some polymer molecules, and the oxidized small molecules generated by the chain scission with hydroxyl, carbonyl, carboxyl hydrophillic groups, and peroxide moiety, dissolved in or were removed by the distilled water + IPA. Thus, the peroxide concentration detected was decreased by washing the gaseous ozonated membrane.

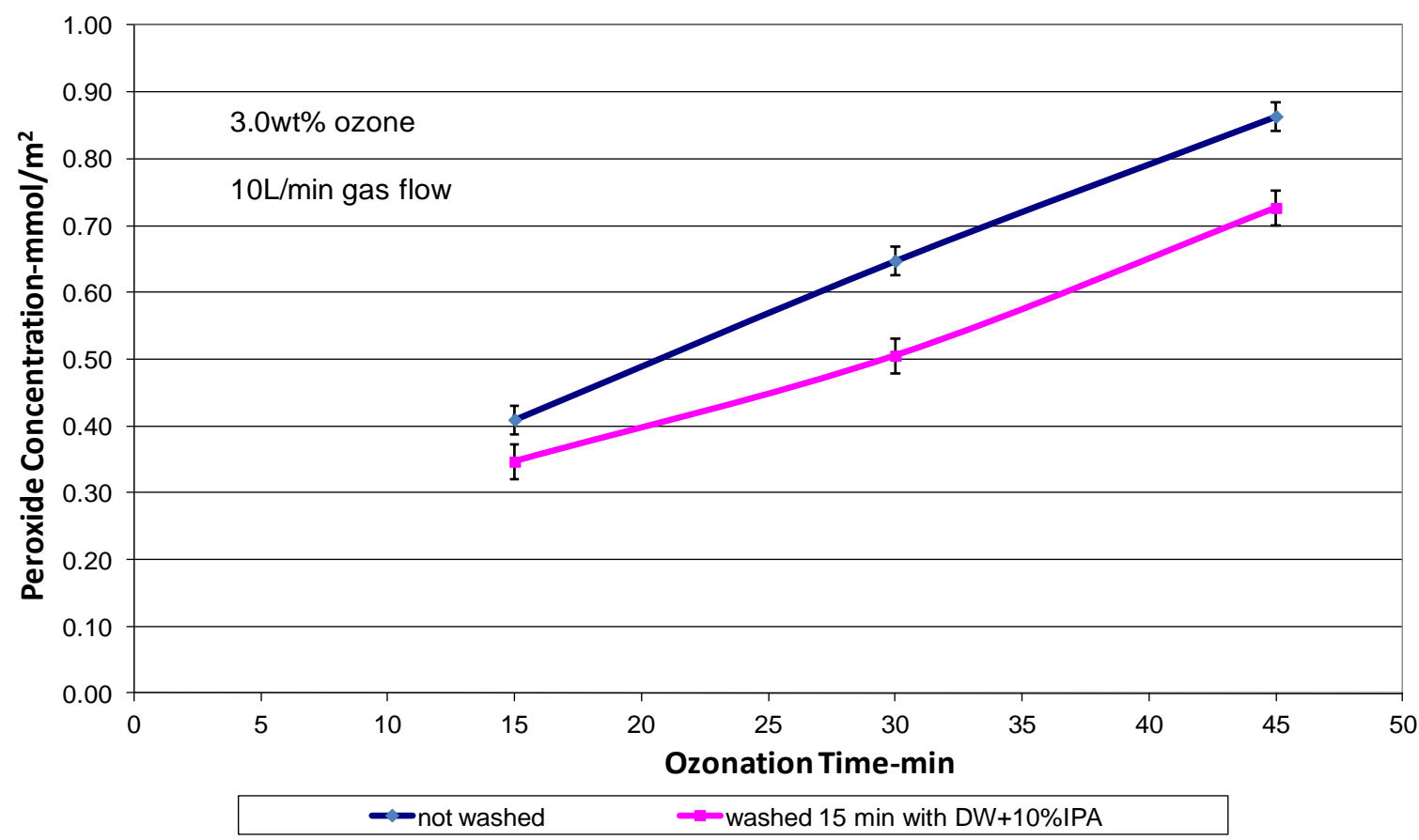

Fig 4.2 Comparison of the peroxide concentrations of PPMM ozonated in gaseous phase with and without washing 
As shown in Figure 4.3, the PPMM that was ozonated in aqueous phase was washed using distilled water plus IPA and compared to the non-washed PPMM membrane that was ozonated in aqueous phase. It was found that the peroxide concentration of the washed PPMM was very close to that of the non-washed PPMM. This indicated that there were not many oxidized small molecules remaining on the surface after the aqueous ozonation.

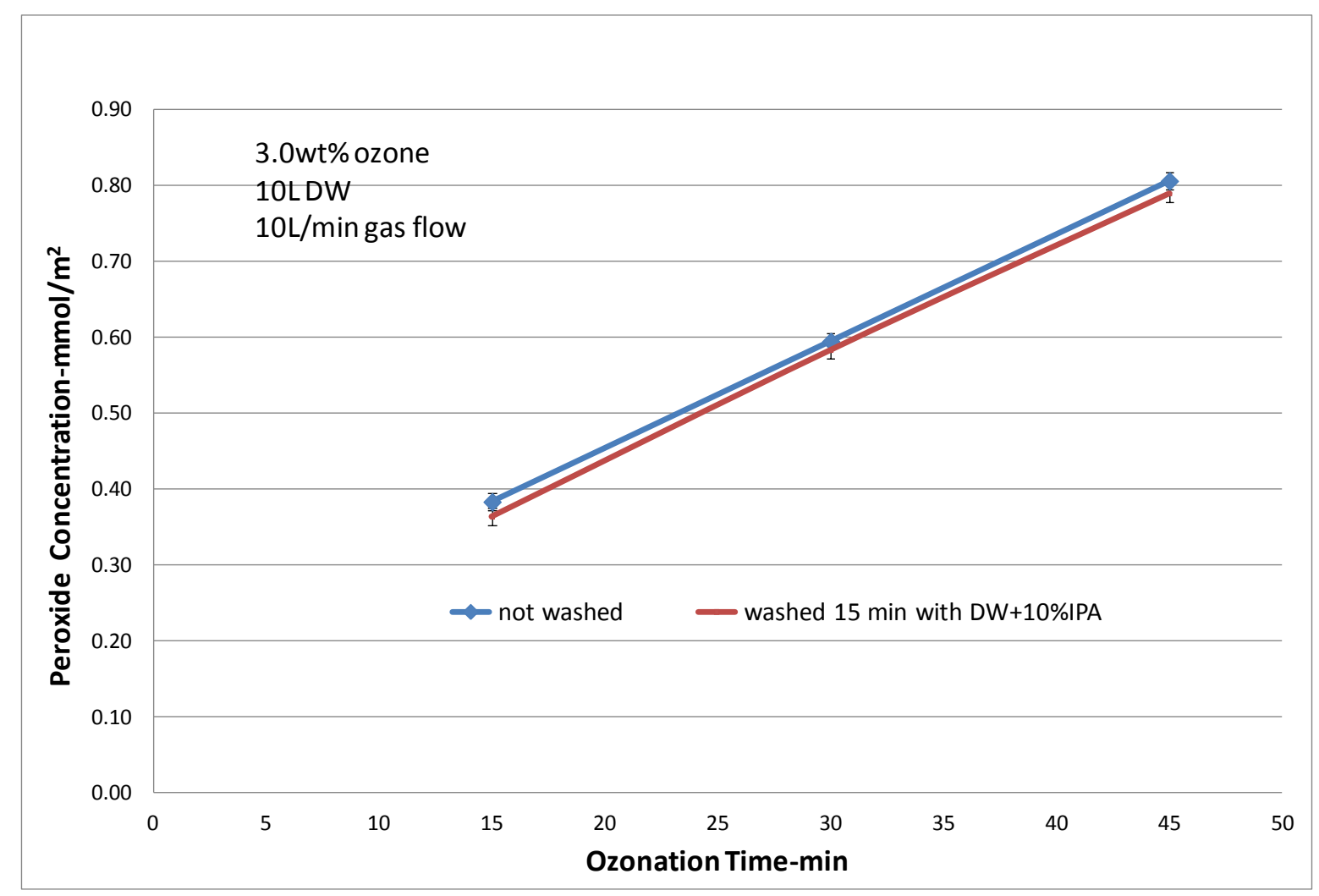

Fig 4.3 Comparison of peroxide concentration of PPMM ozonated in aqueous solution with and without washing

The above tests explain the reason in the following graft tests that the detected peroxide concentration of the PPMM that underwent gaseous phase ozonation was higher, but the AAm graft polymerization was lower than its aqueous counterpart.

Mechanism schematic is as shown in Figure 4.4, when the peroxide group was generated on the backbones of large molecules, they remained on the membranes and initiated the graft polymerization. 


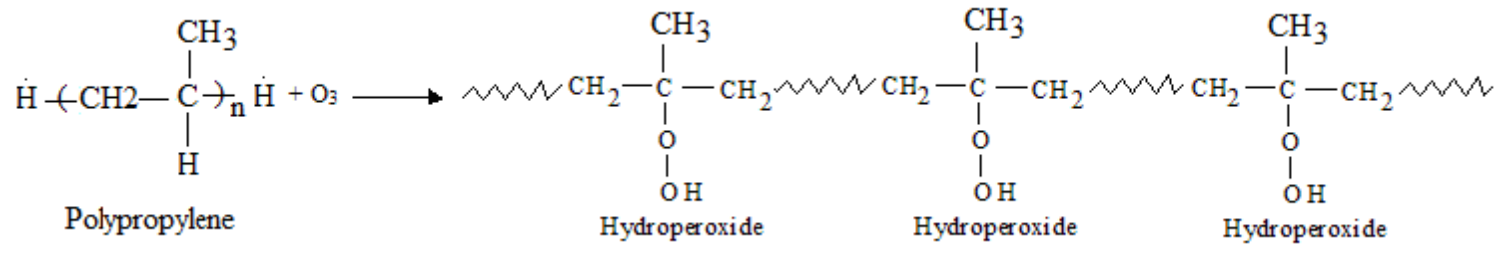

Figure 4.4 Ozone reaction with PPMM to create hydroperoxide

As shown in Figure 4.5, when the peroxide group was generated on smaller scission molecules, it was washed away by water, monomer solution, or by the cleaning procedure:

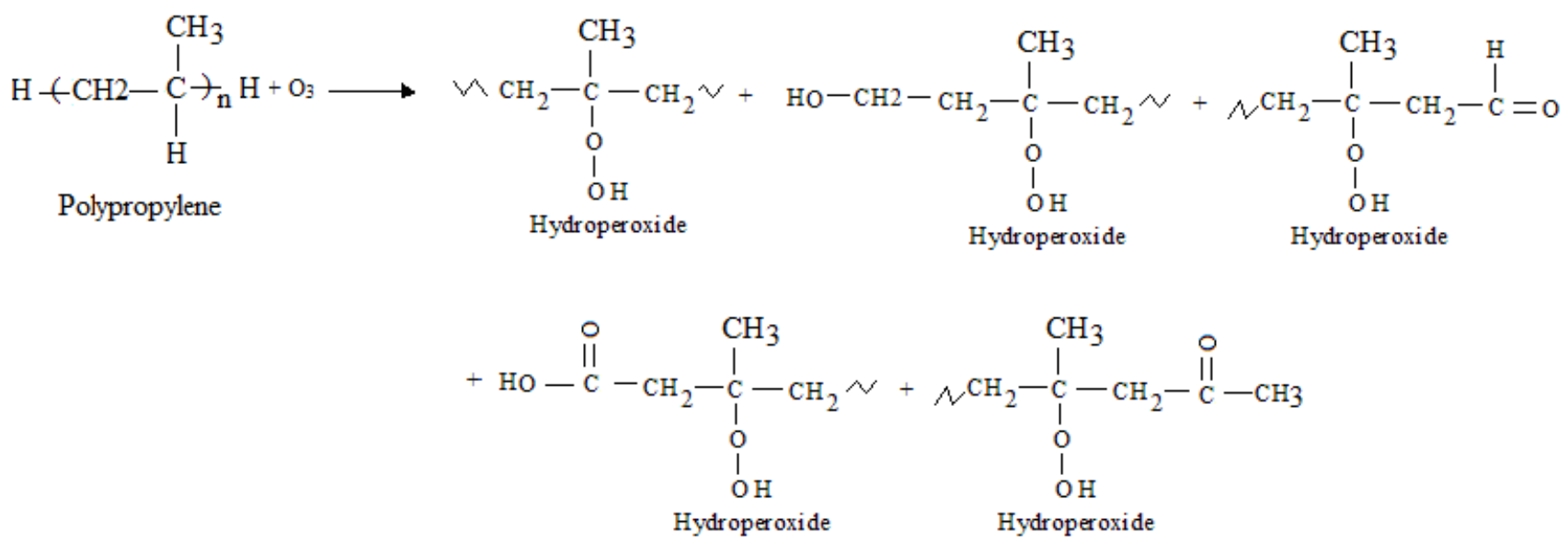

Figure 4.5 Ozone reacted with PPMM to create small molecules by chain scission

\subsubsection{Pre-wet test}

PP membrane is highly hydrophobic. The surface cannot be wetted by distilled water; it can be well wetted when pre-soaked in isopropanol alcohol (IPA) for 15 seconds and then soaked in distilled water for 5 minutes.

In this test, dry and pre-wetted membranes were employed in the aqueous phase ozonation at the same dose and time period, and the amounts of peroxide generated were compared as shown in Figure 4.5. It was found during the first 45 minutes that the dry membranes had a little lower peroxide concentration than the pre-wetted ones, but the difference was small. After 45 minutes, the peroxide concentrations of the dry membranes were higher than those of the pre-wetted ones. This phenomenon can be explained by the fact that the pre-wetted samples had a higher probability of the surface making contact with the 
oxide groups $\left(\cdot \mathrm{OH}\right.$, dissolved $\left.\mathrm{O}_{3}\right)$ in water when the reaction started. After some time during which oxidation occurred, the surface hydrophilicity of the dry samples improved, and both the hydroxyl radicals and the dissolved ozone were able to make contact with the surface of the PPMM samples; and thus the reaction speed increased.

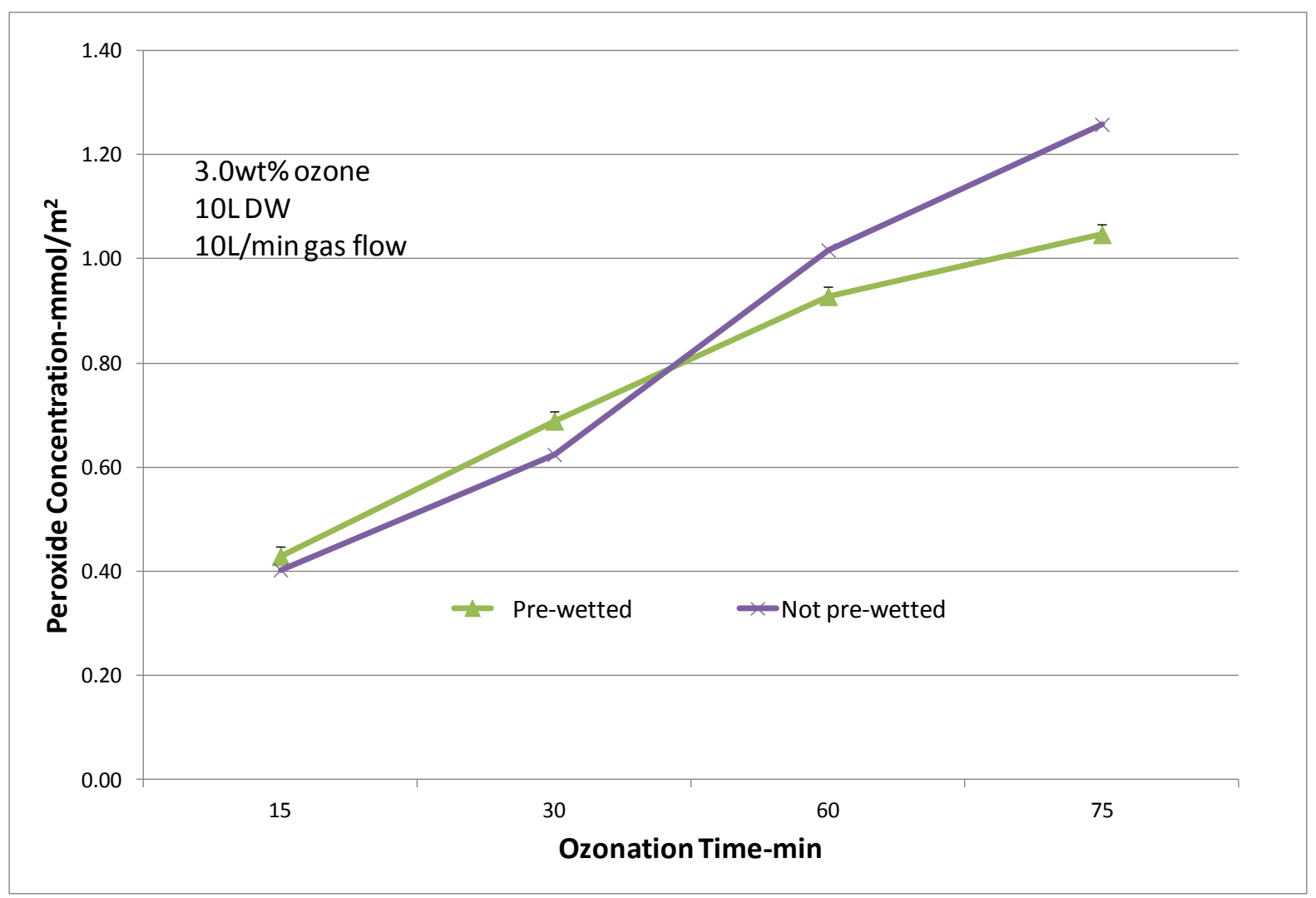

Fig 4.6 Results of aqueous phase ozonation of pre-wetted PPMM

Because the difference in peroxide concentrations was small for the ozonation time of 15-45 minutes (which was used for graft polymerization) and the removal of the IPA that was present in the pre-wetting membrane cannot be assured to be $100 \%$, in order to avoid introducing impurities into the ozonation phase, only the dry membranes were used in the subsequent tests.

\subsubsection{Ozone dose test}

Different ozone doses were used in the aqueous phase to compare the peroxide concentration generated. The results are shown in Figure 4.7. Results indicated that as long as 
the ozonation time multiplied the ozone concentration were equal, the peroxide concentrations came out almost the same. Based on these results, the $3.0 \mathrm{wt} \%$ ozone was arbitrarily selected for the ozonation in all of this research.

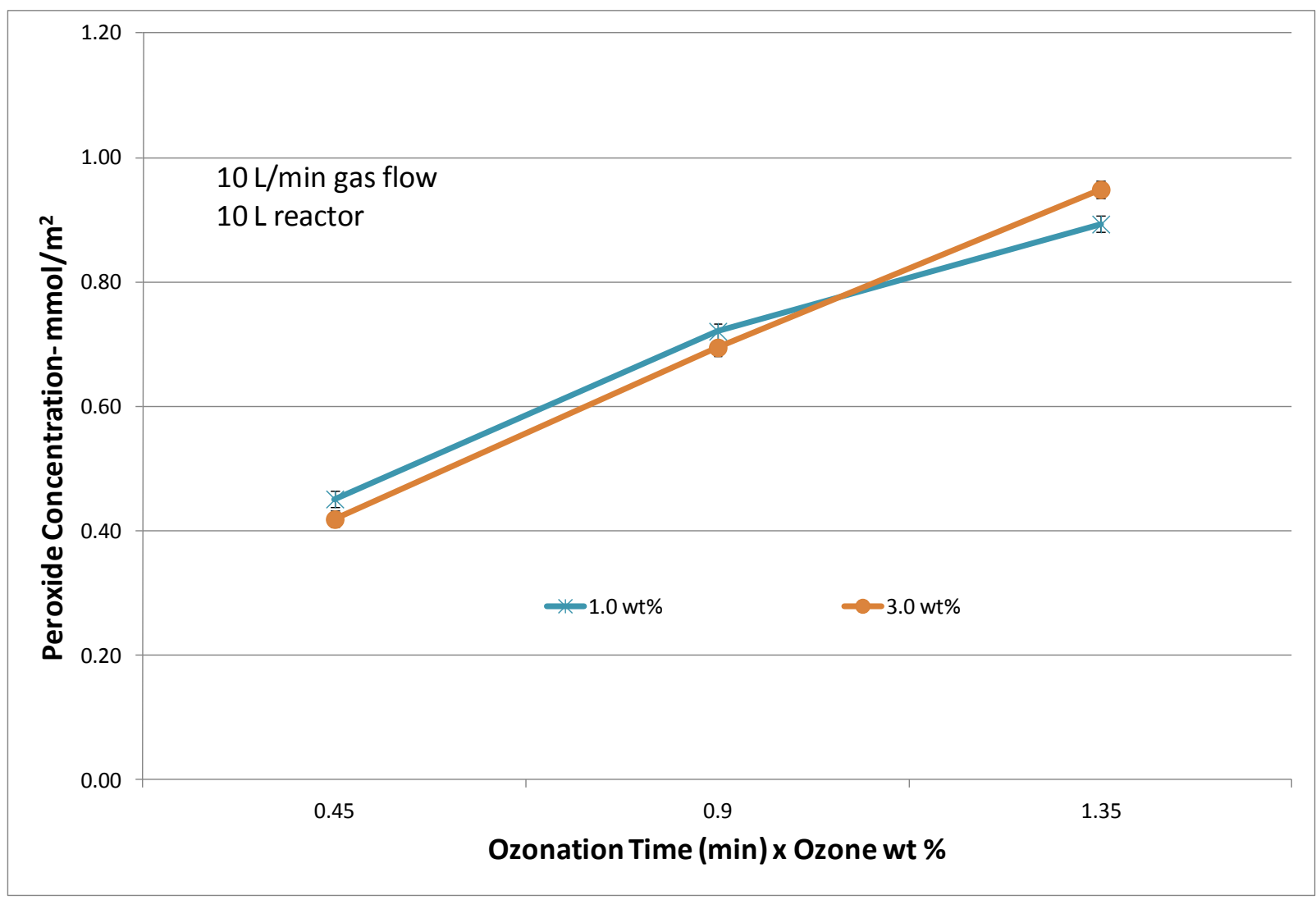

Fig. 4.7 Different ozonation doses - $1.0 \mathrm{wt} \%$, and $3.0 \mathrm{wt} \%$ - applied in the aqueous phase

\subsubsection{Scavenger test}

It is well known that ozone self-decomposes in water to form hydroxyl radical (Beltran, 2000; Gottschalk, 2000; Hewes et al., 1971). In the aqueous phase, both molecular ozone and free radicals are present. In order to find whether both of them contributed to the generation of peroxide, radical scavenger tests were conducted. Radical scavengers are chemicals that capture the radicals generated during the reaction (Bruice et al., 2004; Murakami et al., 2005). The results of the scavenger test are shown in Figure 4.8. 3g/L sodium carbonate was added to the distilled water to eliminate the free radicals. It was demonstrated that both the hydroxyl radicals and the ozone molecules contributed to the oxidation. The free radicals were responsible for $10-25 \%$ (The peroxide concentration was 
reduced from 1.401 to $1.067 \mathrm{mmol} / \mathrm{m}^{2}$, or $24 \%$, at $90 \mathrm{~min}$ of ozonation; and to $12.30 \%$ after 15 min of ozonation.) of the contribution to the peroxide generation, and the percentage of free radicals increased with ozonation time.

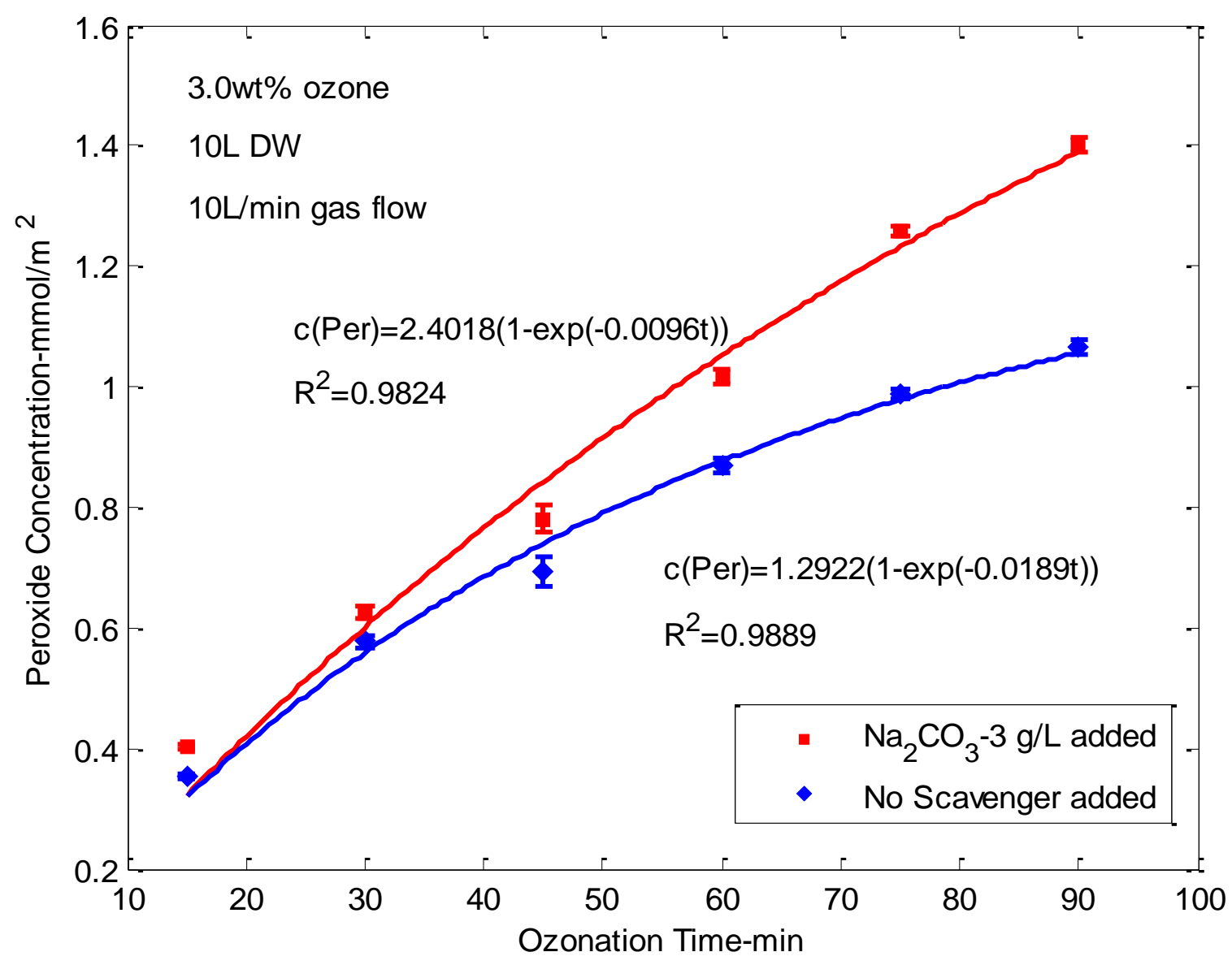

Fig 4.8 Peroxide generation of PPMM ozonated in distilled water with sodium carbonate as scavenger at $3 \mathrm{~g} / \mathrm{L}$ concentration

\subsection{6 pH test}

It is known that aqueous ozonation reactions are affected by $\mathrm{pH}$ level. The effect of $\mathrm{pH}$ on ozonation to organic solute (dissolved in waste water) and ozone decay have been broadly investigated by other researchers at different $\mathrm{pH}$ ranges, the results came out different in reaction order by different researcher (Table 2.5 in Section 2.3.2 showed the reported reaction orders for the decay rate of ozone). It is necessary to investigate the $\mathrm{pH}$ effect on the ozonation of membranes and films (not dissolved in water) to find the reaction trend. In this research the $\mathrm{pH}$ range for PPMM ozonation was 3.0 to 10.0 , as adjusted by $1 \mathrm{M} \mathrm{H}_{2} \mathrm{SO}_{4}$ and 
$1 \mathrm{M} \mathrm{NaOH}$. As shown in Figure 4.9, the peroxide concentration varies with $\mathrm{pH}$. In general peroxide generation slightly decreased with $\mathrm{pH}$. Then different ozonation times were used with $\mathrm{pH}$ of 2.3 and 10.0. It was demonstrated in Figure 4.10 that at a lower $\mathrm{pH}$ environment, there was a higher peroxide content generated.

Conducting ozonation at different $\mathrm{pH}$ levels yielded information about reaction mechanisms. For example, as shown in Section 2.3.2, Beltran et al. found that the rate of ozone decomposition reaction noticeably grew as $\mathrm{pH}$ was raised, which was due to the catalyzing effect of the hydroxide ions $-\mathrm{OH}^{-}$. The mechanism includes a chain of successive reactions yielding $\mathrm{H}_{2} \mathrm{O}_{2}, \mathrm{O}_{3}{ }^{-}, \cdot \mathrm{OH}$ and $\mathrm{HO}_{2} \cdot$ radicals (Beltran, 2004). Even though the dissolved ozone concentration decreased in the higher $\mathrm{pH}$ environment, the generated radicals have a higher reaction activity, thus benefit the oxidation of the organic contents in the waste water. This mechanism is correct for the ozonation of dissolved organic substrates. However, the results in our research showed different trend that in regard to the peroxide generation of the membrane or film ozonation. The three oxidants in aqueous ozonation: the gaseous ozone in bubbles absorbed on the membranes and film surfaces, the dissolved molecular ozone and the free radicals from ozone decomposition in water, all contribute to the peroxide generation. However, in this study, it seems that the gaseous ozone and the dissolved molecular ozone contributed more in peroxide generation than the free radicals. Since ozone is only slightly soluble in water, the resistances to mass transfer of ozone in gaseous phase and interface are insignificant, the main resistance is in the aqueous phase. All three oxidants have to be transferred to the surfaces of membranes and films first and then they can generate peroxides. Although the radicals are stronger oxidants than the gaseous ozone and dissolved molecular ozone, their existed time, when generated from the ozone decomposition, is very short-less than $10 \mu$ s (Gottschalk et al., 2000), and most of them was eliminated on the way to migrate to the membrane or film surface, only the radicals in contact with the membranes or films did the oxidation. While the molecular ozone in water can migrate to the reactive surface, and the gaseous ozone is absorbed on the surface, they have much longer life time. The slightly slow down of the peroxide generaton with $\mathrm{pH}$ was the overall effect of the decreased dissolved molecular ozone concentration and the increased formation of radicals from the decomposition of molecular ozone. 


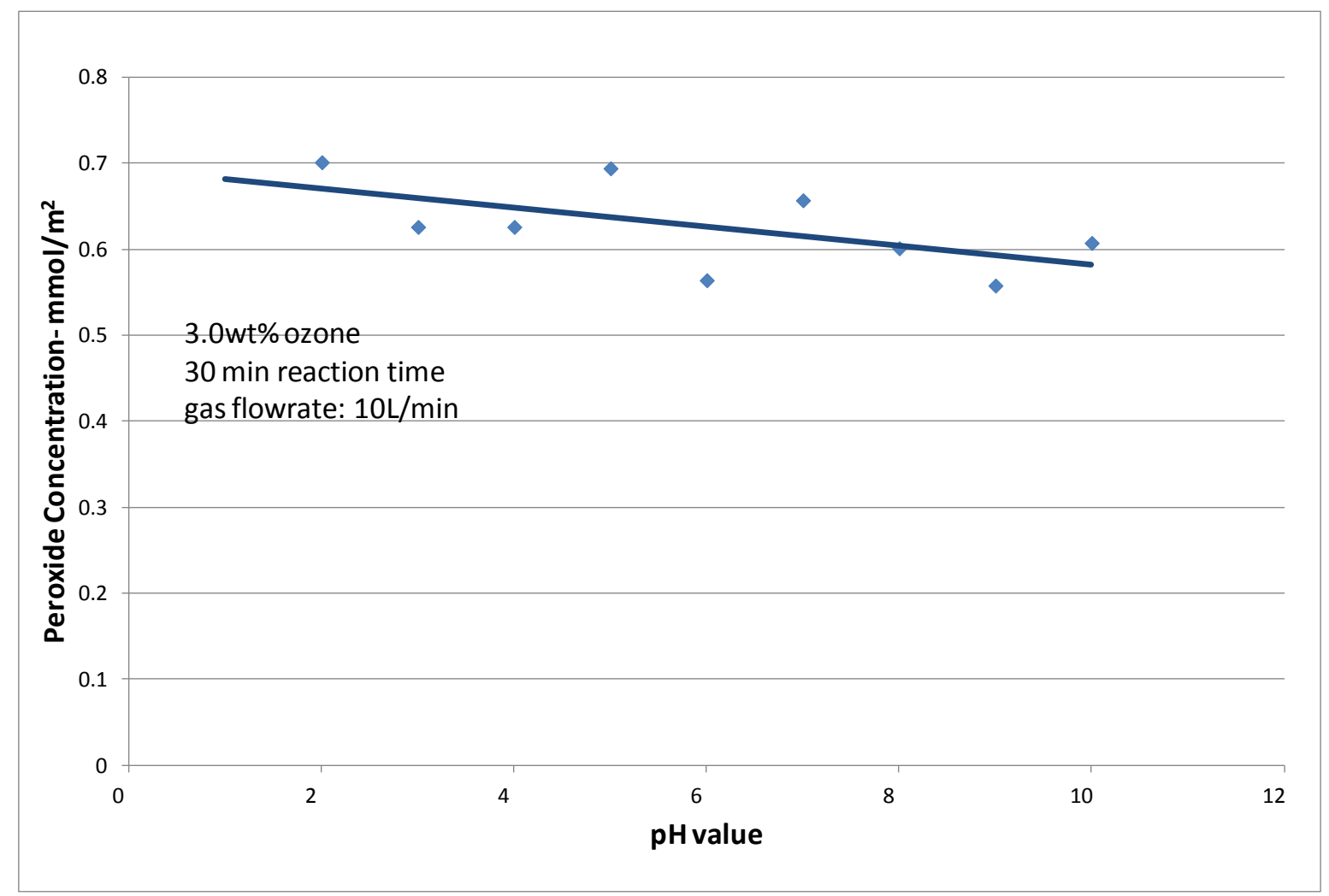

Fig 4.9 Peroxide generation at different $\mathrm{pH}$ levels with 3.0wt $\%$ of applied ozone dose and 30 min ozonation

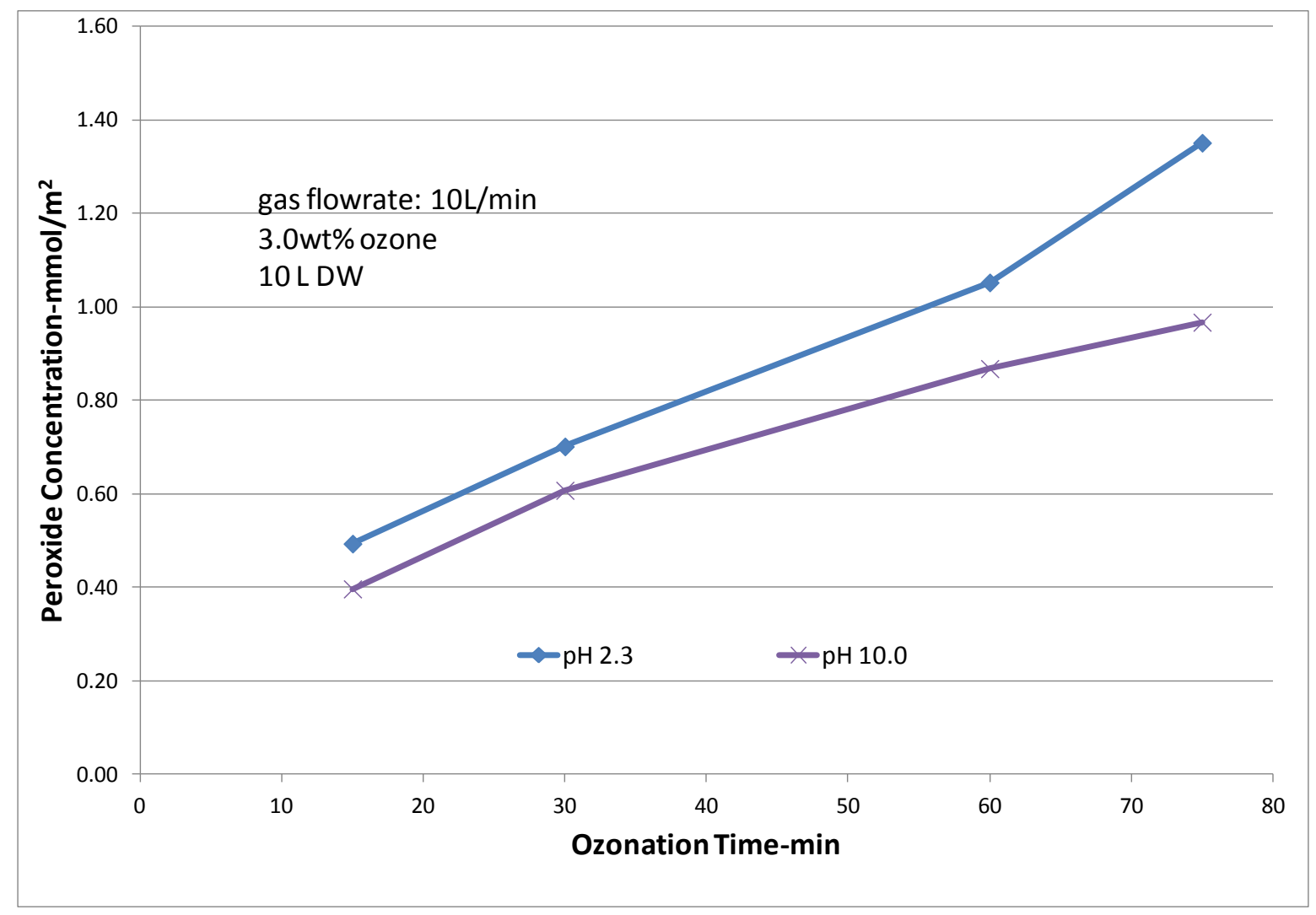

Fig 4.10 Peroxide generation with different ozone doses applied at different $\mathrm{pH}$ levels 


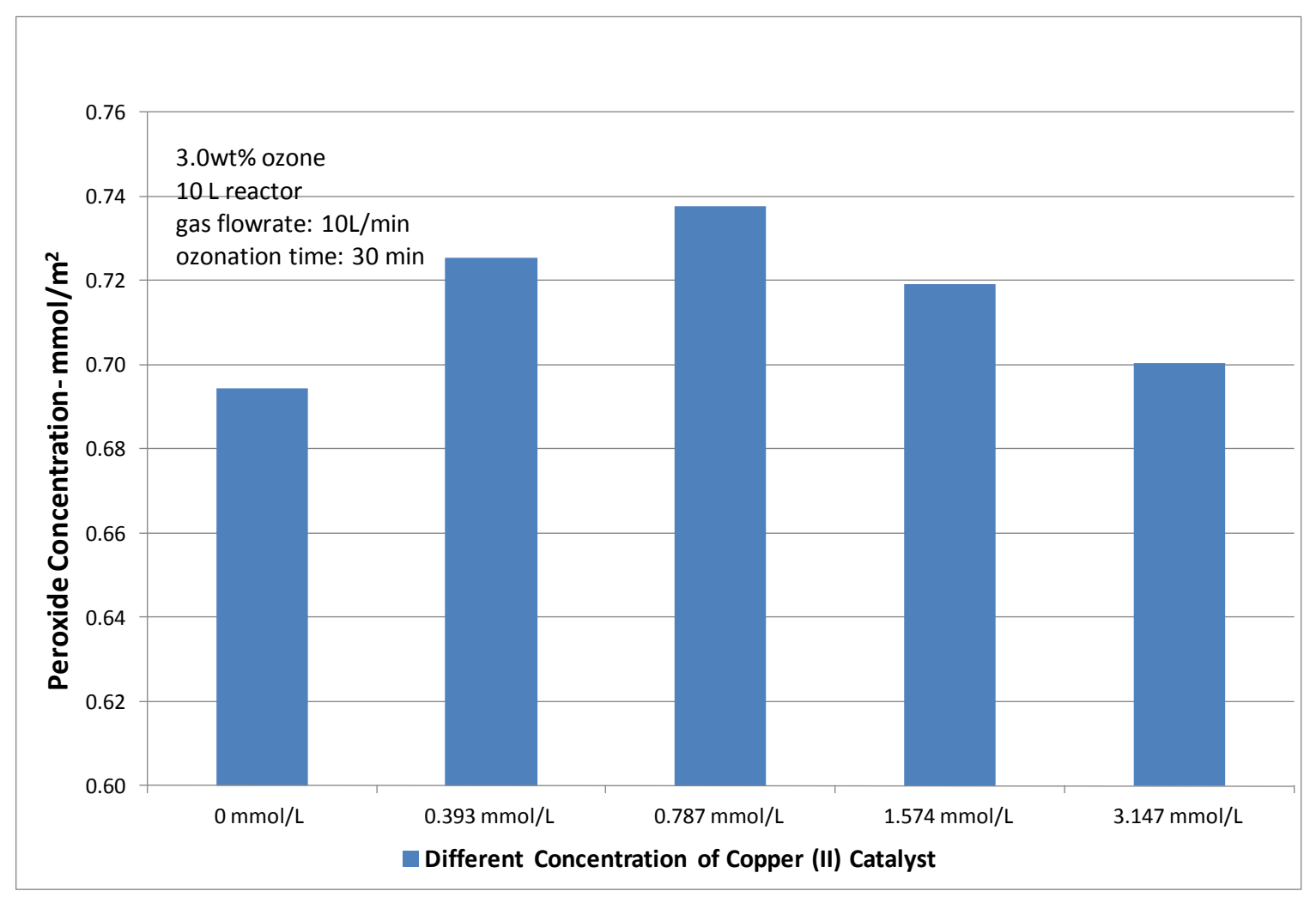

Fig 4.11 Peroxide generation of ozonation for 30 minutes and at $3.0 \mathrm{wt} \%$ using $\mathrm{Cu}^{2+}$ catalyst in DW

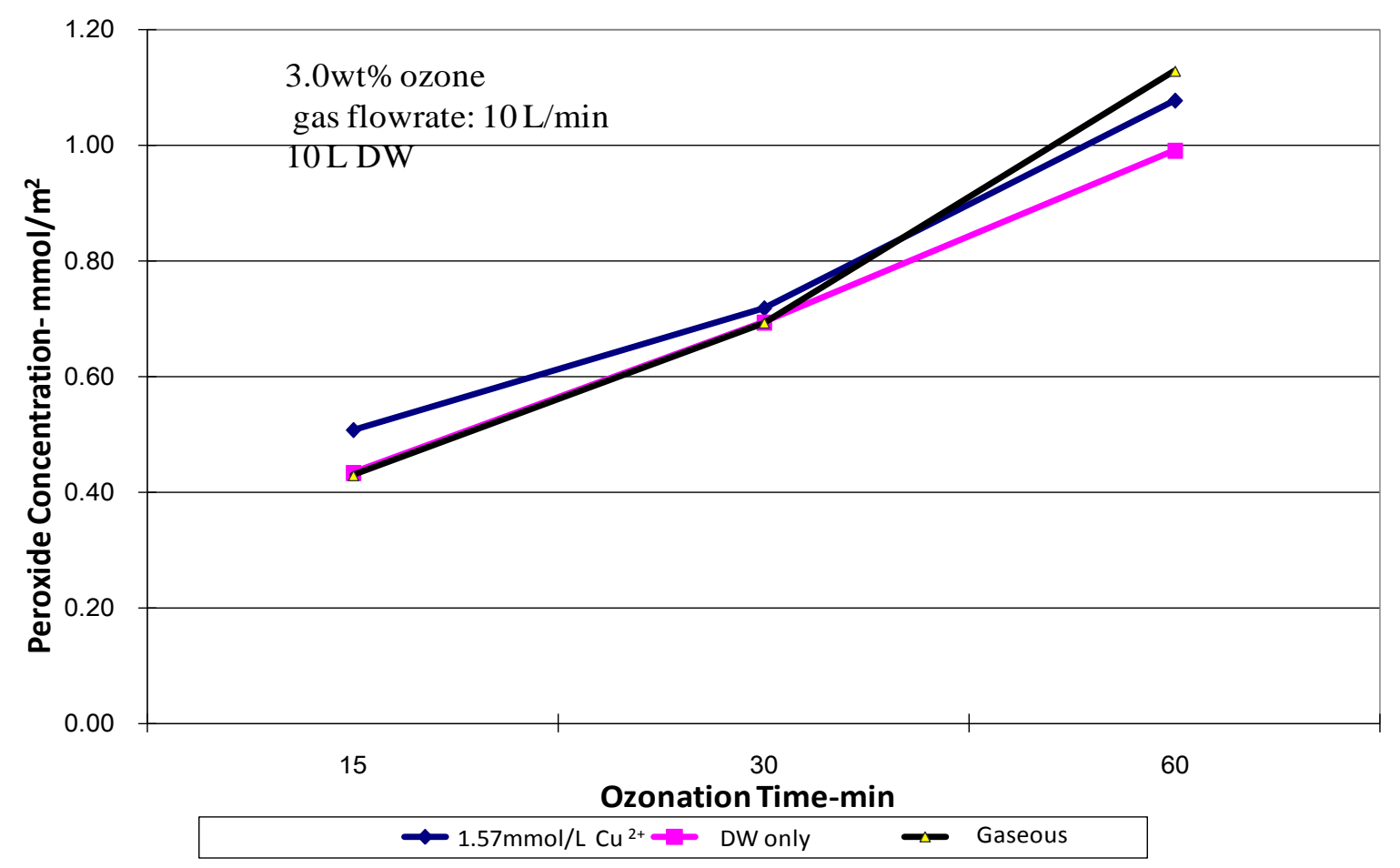

Fig 4.12 Peroxide generation at different ozonation time using $\mathrm{Cu}^{2+}$ catalyst in DW and in the gaseous phase 


\subsubsection{Homogeneous catalyst test $-\mathrm{Cu}^{2+}$}

According to our group member's (Mastan E., et al., 2013) research, $\mathrm{Cu}^{2+}$ can accelerate peroxide generation on polyethylene films undergoing ozonation. The catalytic reaction applied to PPMM was investigated in this study at the 0.5 hour ozonation time and $3.0 \mathrm{wt} \%$ ozone gas mixture in the aqueous phase with $\mathrm{Cu}^{2+}$ ion of $0.3934 \mathrm{mmol} / \mathrm{L}, 0.7868$ $\mathrm{mmol} / \mathrm{L}, \quad 1.5737 \mathrm{mmol} / \mathrm{L}$, and $3.1473 \mathrm{mmol} / \mathrm{L}$ of $\mathrm{CuSO}_{4} \cdot 5 \mathrm{H}_{2} \mathrm{O}$, respectively. The concentration of generated peroxide was investigated, as shown in Figure 4.11. It was found that the $0.7868 \mathrm{mmol} / \mathrm{L}$ concentration of $\mathrm{Cu}^{2+}$ ion resulted in the highest increase in peroxide generation, and the lower $(0.3934 \mathrm{mmol} / \mathrm{L})$ and the higher concentrations $(1.5737 \mathrm{mmol} / \mathrm{L}$ or $3.1473 \mathrm{mmol} / \mathrm{L}$ ) of $\mathrm{Cu}^{2+}$ ion contributed positively to the peroxide generation, but the effectiveness was lower.

When $1.5737 \mathrm{mmol} / \mathrm{L} \mathrm{Cu}^{2+}\left(\mathrm{CuSO}_{4} \cdot 5 \mathrm{H}_{2} \mathrm{O}\right)$ were added to distilled water, and PPMM was ozonated for different time periods at $3.0 \mathrm{wt} \%$ ozone concentration in the aqueous phase, the results indicated that catalytic ozonation using $\mathrm{Cu}^{2+}$ increased the peroxide generation by $17.14 \%$. Figure 4.12 shows the results (peroxide $0.5083 \mathrm{mmol} / \mathrm{m}^{2}$ vs $0.4340 \mathrm{mmol} / \mathrm{m}^{2}$ at 15 minutes).

These results indicated that the concentration of $\mathrm{Cu}^{2+}$ ion could accelerate the peroxide generation for PPMM; and the concentration of $\mathrm{Cu}^{2+}$ ion that resulted in the best catalytic results was determined. Further investigation was conducted, as discussed in Section 4.18 to find the mechanism.

As shown in Figure 4.12, the $\mathrm{Cu}^{2+}$ catalytic ozonation in the aqueous phase resulted in a higher concentration of peroxide than that conducted in the DW ozonation; and there was a higher peroxide generation than in the gaseous phase ozonation in the 15 and 30 minute reactions.

$\mathrm{pH}$ value changed slightly when different concentrate of $\mathrm{CuSO}_{4} \cdot 5 \mathrm{H}_{2} \mathrm{O}$ was added into the water. In our experiments, the $\mathrm{pH}$ value changed from 4.65 to 4.20 when $\mathrm{Cu}^{2+}$ concentrate changed from $0.3934 \mathrm{mmol} / \mathrm{L}$ to $3.1473 \mathrm{mmol} / \mathrm{L}$. Therefore the $\mathrm{pH}$ value was not a significant factor that affected this experiment. 


\subsubsection{Investigative tests of the mechanism in homogeneous catalyst $\mathrm{Cu}^{2+}$}

The author did not find any literature that indicated the mechanism in the use of $\mathrm{Cu}^{2+}$ catalyst in ozonation. The mechanism was investigated in this research, and an enhanced radical production process was indicated.

\subsubsection{Application of scavengers to the $\mathrm{Cu}^{2+}$ catalytic ozonation}

Tests using the scavenger $\mathrm{Na}_{2} \mathrm{CO}_{3}$ in $\mathrm{Cu}^{2+}$ catalytic ozonation in the aqueous phase were conducted, $1.5737 \mathrm{mmol} / \mathrm{L} \mathrm{Cu}^{2+}\left(\mathrm{CuSO}_{4} \cdot 5 \mathrm{H}_{2} \mathrm{O}\right)$ was added to distilled water, and 3.0 $\mathrm{g} / \mathrm{L} \mathrm{Na}_{2} \mathrm{CO}_{3}$ (Wu et al., 2000) was used as scavenger. The PPMM was ozonated using an ozone dose of $3.0 \mathrm{wt} \%, 10.0 \mathrm{~L} / \mathrm{min}$ gas flow rate for 30 minutes in the $10 \mathrm{~L}$ reactor. The concentration of peroxide generated is shown in Figure 4.13. It was indicated that after the scavenger was added, the peroxide generation was dropped to almost the same level no matter there was a catalyst or not. This phenomenon strongly supported our conclusion that the $\mathrm{Cu}^{2+}$ catalyzed ozonation by increasing the concentration of radicals.

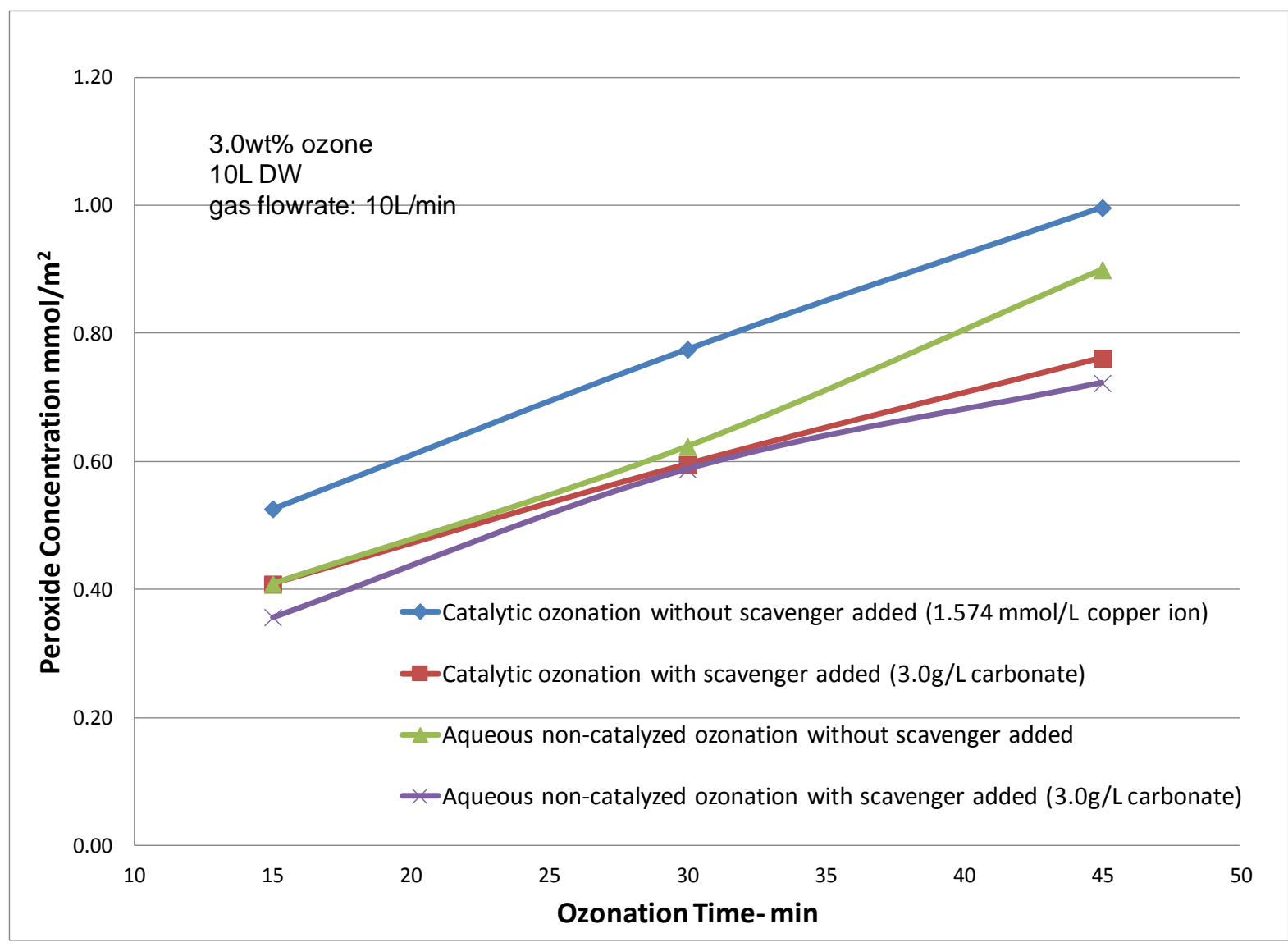

Fig 4.13 Scavenger's effect on $\mathrm{Cu}^{2+}$ catalytic ozonation with $3.0 \mathrm{~g} / \mathrm{L} \mathrm{Na}_{2} \mathrm{CO}_{3}$ added 
A different scavenger, $0.1 \mathrm{~mol} / \mathrm{L}$ t-Butanol, was also added to the $\mathrm{Cu}^{2+}$ catalytic ozonation. As shown in Figure 4.14, at 30 and 45 minutes, peroxide generation also dropped to almost the same level as that observed when $\mathrm{Na}_{2} \mathrm{CO}_{3}$ was used in the aqueous phase ozonation. It was confirmed by using an alternative scavenger that $\mathrm{Cu}^{2+}$ catalyzed ozonation of PPMM by increasing radical concentration.

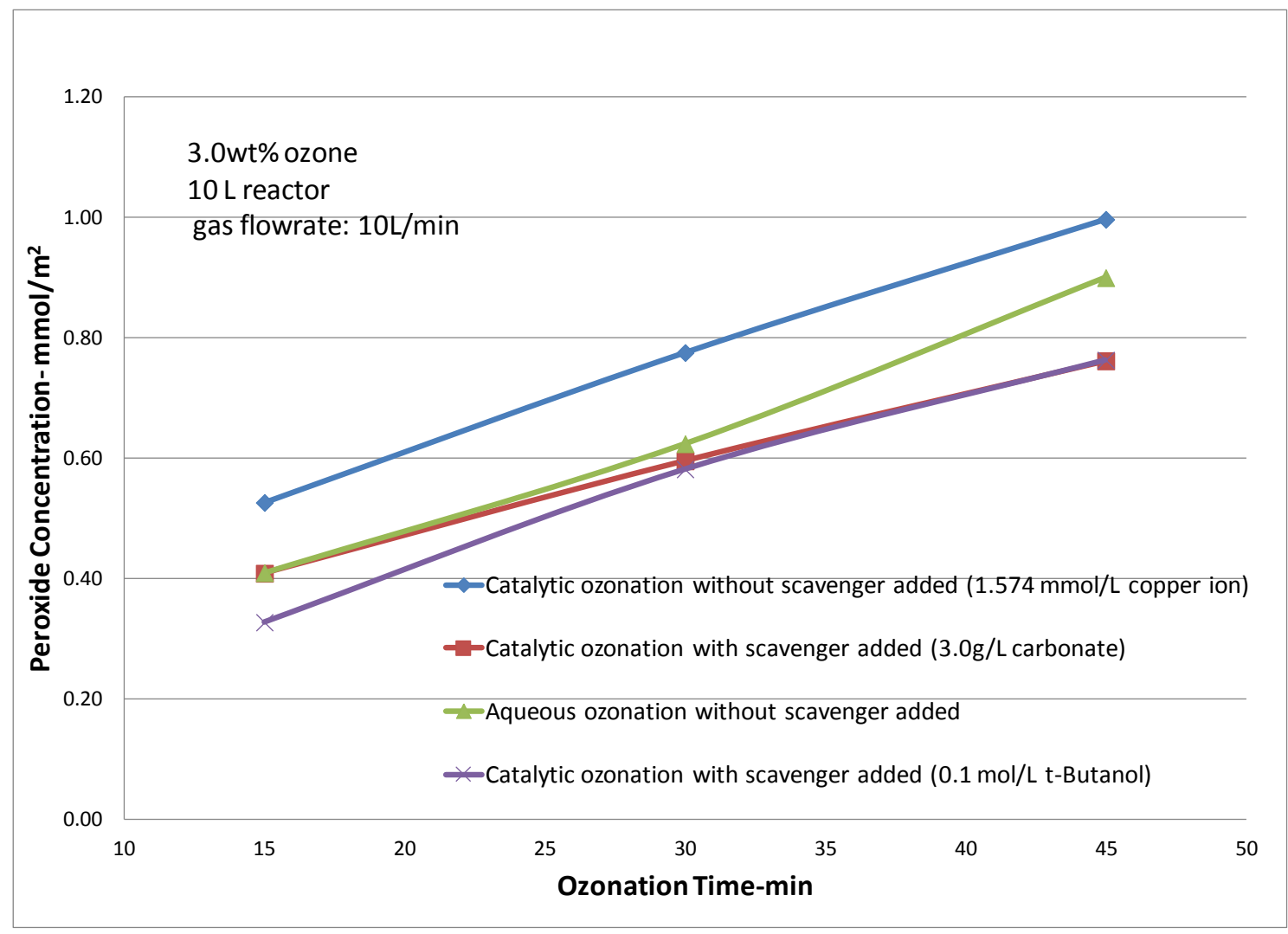

Fig 4.14 The effect of scavengers on $\mathrm{Cu}^{2+}$ catalytic ozonation at room temperature

Scavenger tests were also conducted on $\mathrm{Cu}^{2+}$ catalytic ozonation at $0{ }^{\circ} \mathrm{C}$. In Section 2.3.2, Equation (2-10) indicated the decomposition of ozone becomes faster as temperature rises (Ershov et al., 2008). It is necessary to test if the catalytic mechanism were the same at different temperature. The same doses of ozone, catalyst, and scavenger as used in the above tests were applied, and the temperature was decreased to $0{ }^{\circ} \mathrm{C}$. The concentration of peroxide generated is shown in Figure 4.15. It was indicated that $\mathrm{Cu}^{2+}$ catalytic ozonation again increased the peroxide generation, and the scavengers reduced the peroxide generated at $0{ }^{\circ} \mathrm{C}$. Comparison of Figure 4.14 with Figure 4.15 also indicates that at lower temperature, 
peroxides generated were less than that at higher temperature.

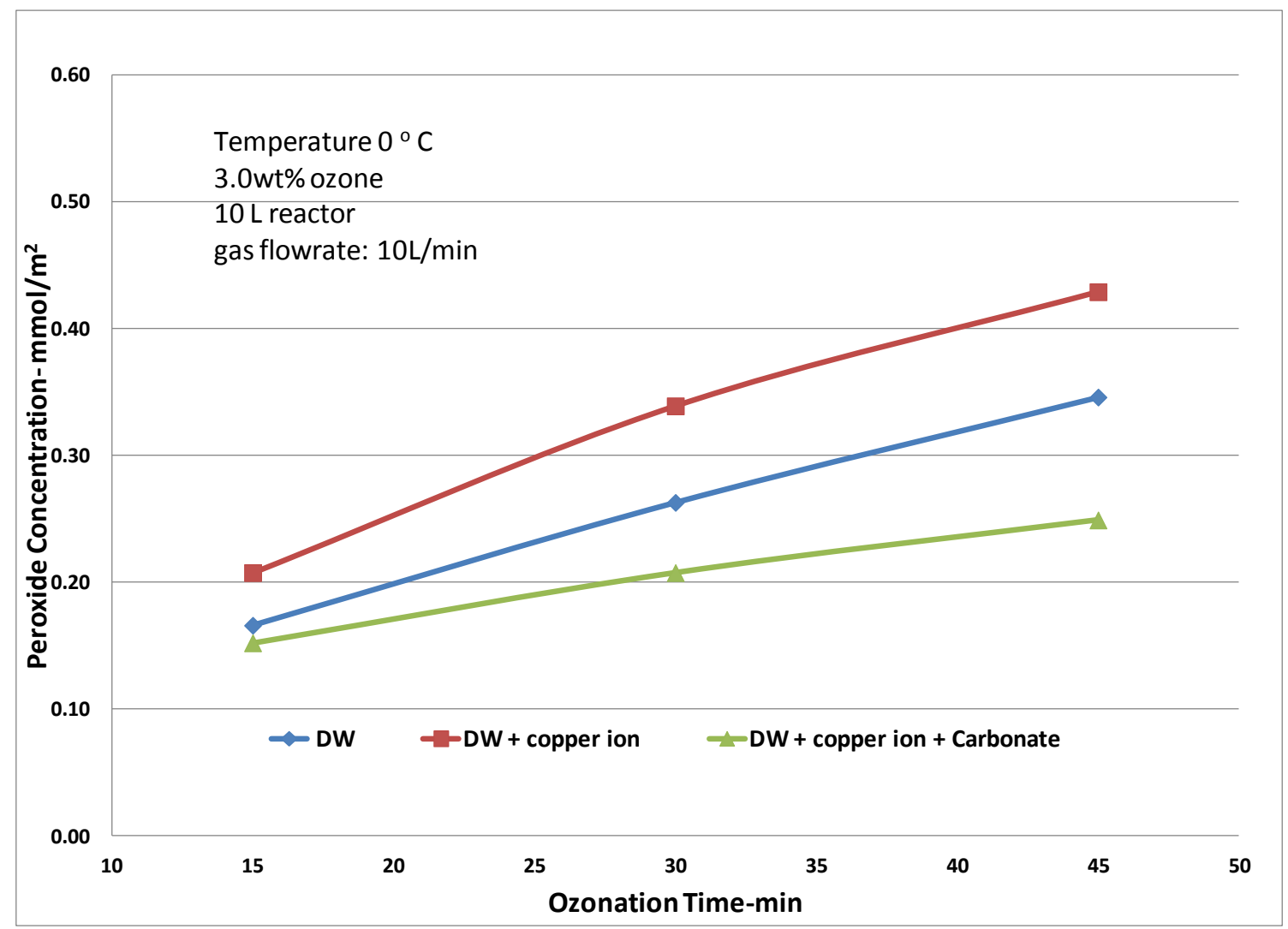

Fig 4.15 The effect of scavengers on $\mathrm{Cu}^{2+}$ catalyzed ozonation at $0^{\circ} \mathrm{C}$

\subsubsection{The effect of anion $\mathrm{SO}_{4}{ }^{2-}$ on the $\mathrm{Cu}^{2+}$ catalytic ozonation}

It was shown in Figure 4.11 that a peak concentration of $\mathrm{Cu}^{2+}$ catalyst was present in catalytic ozonation. Theoretically, when the concentration of $\mathrm{Cu}^{2+}$ catalyst increases, the peroxide concentration should continue to rise or stabilize. The reason the peroxide concentration dropped after reaching a certain level was not known, therefore, the following tests were conducted to find the answer. It is possible that the anions caused the peroxide generation to decline. 


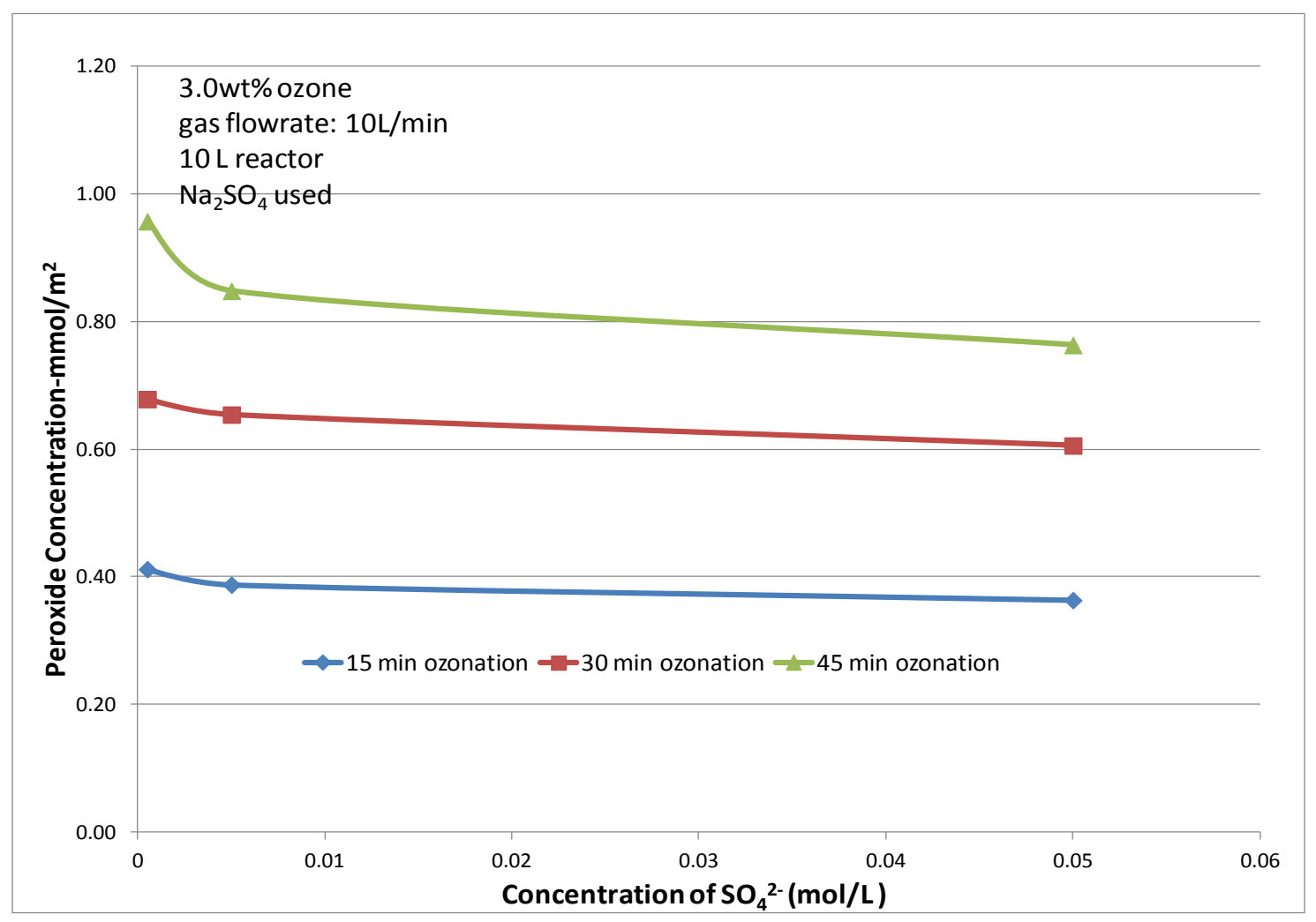

Fig 4.16 Effect of $\mathrm{SO}_{4}{ }^{2-}$ on peroxide generation in non-catalyzed ozonation of PPMM

The effect of the anion $\mathrm{SO}_{4}{ }^{2-}$ (concentration from $5 \times 10^{-4} \mathrm{~mol} / \mathrm{L}$ to $5 \times 10^{-2} \mathrm{~mol} / \mathrm{L}$, which simulated the applied $\mathrm{SO}_{4}{ }^{2-}$ concentration range in the catalyst $\mathrm{CuSO}_{4} \cdot 5 \mathrm{H}_{2} \mathrm{O}$ ) was investigated in aqueous phase ozonation, as shown in Figure 4.16. It was demonstrated that the higher the concentration of $\mathrm{SO}_{4}{ }^{2-}\left(\mathrm{Na}_{2} \mathrm{SO}_{4}\right.$ applied as a provider of anion $\left.\mathrm{SO}_{4}{ }^{2-}\right)$, the less peroxide was generated at the same ozone dose. The explanation is hypothesized by the author to be that the anion $\mathrm{SO}_{4}{ }^{2-}$ reacted with the generated hydroxyl radicals in the same way as $\mathrm{PO}_{4}{ }^{3-}$ and $\mathrm{CO}_{3}{ }^{2-}$ did (Beltran, 2004), and thus its reactivity to generate peroxide was reduced. In Section 4.1.5, Figure 4.8 indicated that the free radicals contributed more to the peroxide generation at the longer ozonation time, the fact that the 45 minutes ozonation curve had a larger drop when the concentration of $\mathrm{SO}_{4}{ }^{2-}$ increased is in agreement with the results of Figure 4.8. The mechanism will be discussed in Section 4.6.3.1. 


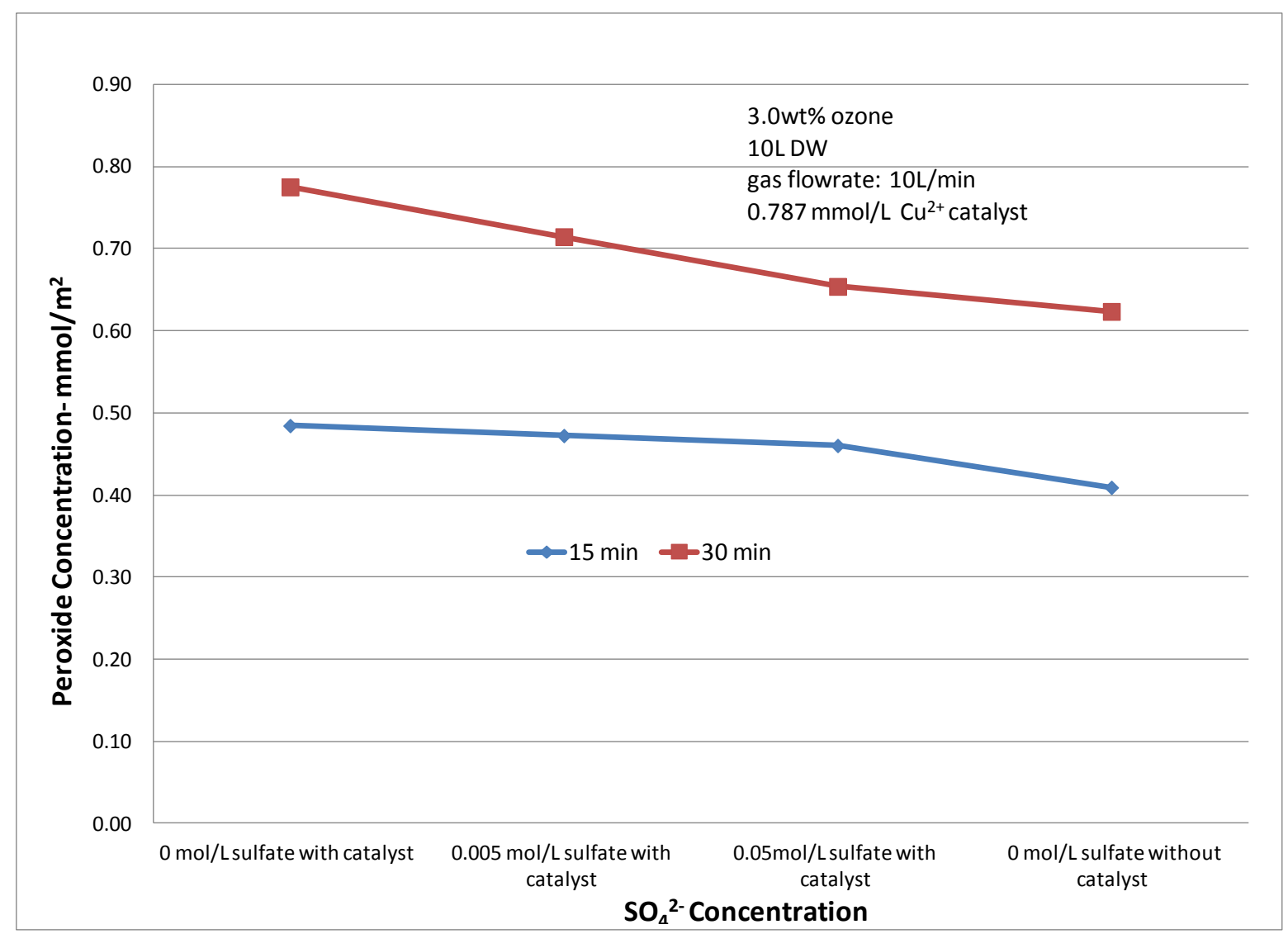

Fig 4.17 Effect of $\mathrm{SO}_{4}{ }^{2-}$ on peroxide generation in catalyzed ozonation of PPMM

To further investigate the effect of $\mathrm{SO}_{4}{ }^{2-}$ on $\mathrm{Cu}{ }^{2+}$ catalytic ozonation, different concentrations of $\mathrm{Na}_{2} \mathrm{SO}_{4}$ were added for 15 and 30 minute periods to $3.0 \mathrm{wt} \% \mathrm{O}_{3}$ and 0.7868 $\mathrm{mmol} / \mathrm{L} \mathrm{Cu}^{2+}$ catalytic ozonation, as shown in Figure 4.17. The peroxide generation decreased when the anion $\mathrm{SO}_{4}{ }^{2-}$ concentration increased; therefore, the negative effect of anion $\mathrm{SO}_{4}{ }^{2-}$ on the $\mathrm{Cu}^{2+}$ catalytic ozonation was confirmed.

\subsubsection{3 $\mathrm{Cu}^{2+}$ catalytic ozonation at a lower temperature $\left(\mathrm{O}^{\circ} \mathrm{C}\right)$}

In Section 2.3.2, Equation (2-10) indicated that the decomposition of ozone becomes faster as temperature rises (Beltran, 2004). A persuasive conclusion should be the outcome of tests conducted in more than one set of conditions. A low temperature $\mathrm{Cu}^{2+}$ catalytic ozonation was thus conducted at $0^{\circ} \mathrm{C}$ for 15 - and 30 -minute periods, respectively, and the investigative results are shown in Figure 4.18. The PPMM was ozonated using the same $\mathrm{O}_{3}$ dose as in Section 4.1.7; however, the temperature was set at $0^{\circ} \mathrm{C}$ instead of room temperature. The results indicate that $\mathrm{Cu}^{2+}$ catalytic ozonation increased the peroxide generation by 
$28.95 \%$ (from 0.2610 to $0.3391 \mathrm{mmol} / \mathrm{m}^{2}$ for $0.7868 \mathrm{mmol} / \mathrm{L} \mathrm{Cu}^{2+}$ and 30 minutes aqueous ozonation). The concentrations of $0.3933 \mathrm{mmol} / \mathrm{L}, 0.7868 \mathrm{mmol} / \mathrm{L}, 1.574 \mathrm{mmol} / \mathrm{L}$, and 3.933 $\mathrm{mmol} / \mathrm{L} \mathrm{Cu}^{2+}$ added to distilled water were also investigated. It was found that 0.7868 $\mathrm{mmol} / \mathrm{L}$ of $\mathrm{Cu}^{2+}$ ion was still the optimal concentration at $0^{\circ} \mathrm{C}$.

As shown in Figure 4.19, $\mathrm{Cu}^{2+}$ catalytic ozonation was compared with the same ozone dose at room temperature and at $0^{\circ} \mathrm{C}$, it was found that although the higher peroxide concentration was generated at higher temperature (it was indicated that the catalytic ozonation was a heat adsorption reaction as well), the two curves had the similar trend with different $\mathrm{Cu}^{2+}$ catalysis concentration, and $0.7868 \mathrm{mmol} / \mathrm{L}$ of $\mathrm{Cu}^{2+}$ ion was the optimal catalyst concentration at both temperature.

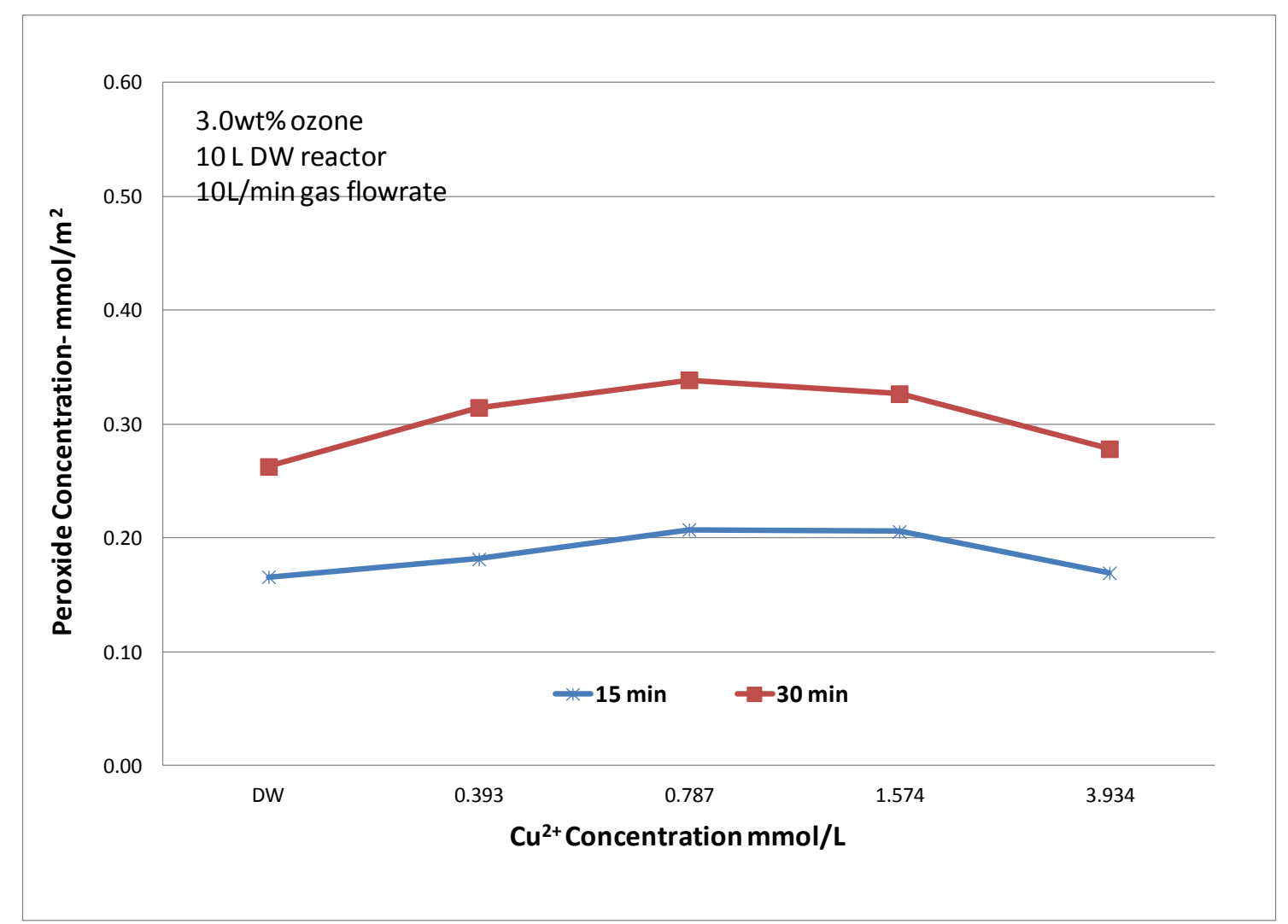

Fig 4.18 Different concentrations of $\mathrm{Cu}^{2+}$ catalyst applied to $0^{\circ} \mathrm{C}$ ozonation at 15 and $30 \mathrm{~min}$ 


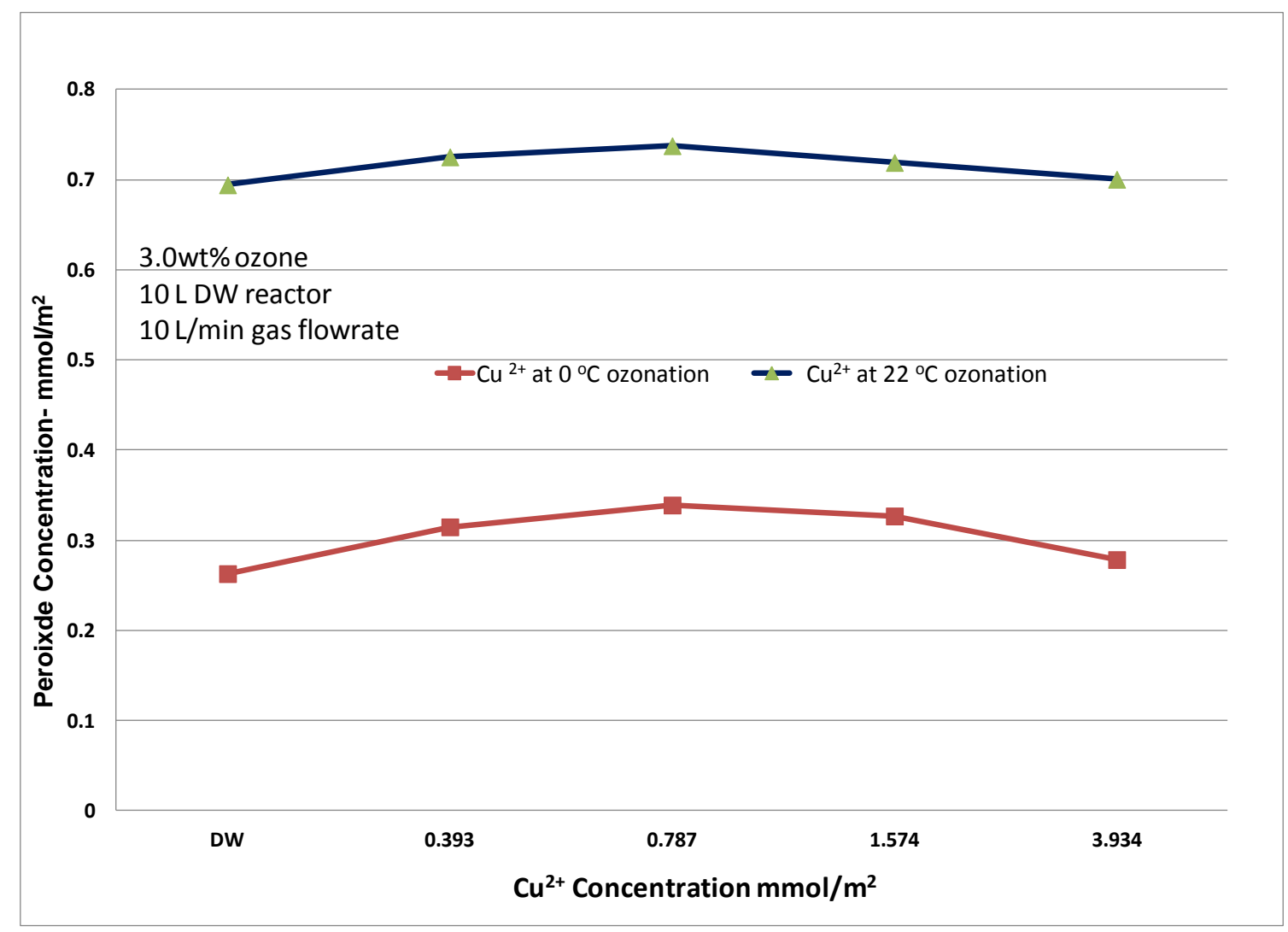

Fig 4.19 Different concentrations of $\mathrm{Cu}^{2+}$ catalyst applied to $0^{\circ} \mathrm{C}$ and $22^{\circ} \mathrm{C}$ ozonation

4.1.8.4 Investigation of dissolved ozone concentration in distilled water undergoing $\mathrm{Cu}^{2+}$ catalytic ozonation

The increase in the amount of radicals generated by $\mathrm{Cu}^{2+}$ catalysis was demonstrated in Sections 4.1.8.1 and 4.1.8.3. How about the dissolved molecular ozone? Is the dissolved molecular ozone concentration changed by the $\mathrm{Cu}^{2+}$ catalysis?

In order to investigate the dissolved ozone concentration, a standard indigo method (APHA, Standard Methods for the Examination of Water and Wastewater) was employed. When potassium indigo trisulfonate reacted with ozone, the ozone attacked the central C-C bond, and the extent of the bleaching was based on the molecular absorption coefficient of ozone (Sonntag, et al., 2012). The method is to pipet $3.0 \mathrm{ml}$ of prepared indigo reagent II (diluted potassium indigo trisulfonate) into a $10 \mathrm{ml}$ volumetric flask and dilute to $10 \mathrm{ml}$. Prepare a volumetric flask for each sample and the blank. Pipet $1.0 \mathrm{ml}$ ozone solution into the flask and shake immediately; determine the absorbance of the blank and the samples at 600 nm UV using a 1-cm cuvette (Markee et al., 2009; Appendix IV). The concentration of 
dissolved ozone in the aqueous ozonation and in the copper catalytic ozonation was measured, as shown in Table 4.1. The results demonstrated that there was no significant difference between the concentration of dissolved ozone in the non-catalyzed aqueous ozonation and the catalytic ozonation, and that the ozone concentration was not the factor that caused the difference in the amount of peroxide generated in the distilled water ozonation and the catalytic ozonation.

Table 4.1 Dissolved ozone concentration in pure distilled water in catalytic ozonation

\begin{tabular}{l|c|ccccccc}
\hline \multirow{2}{*}{$\begin{array}{l}\text { Pure DW } \\
\text { Ozonation }\end{array}$} & $\begin{array}{c}\mathrm{O}_{3} \text { gas lead in } \\
\text { Time }(\mathrm{min})\end{array}$ & 0 & 1 & 3 & 5 & 12 & 15 & 25 \\
\cline { 2 - 9 } & $\mathrm{mg} / \mathrm{L} \mathrm{O}_{3}$ & 0.00 & 2.17 & 8.70 & 9.56 & 8.90 & 8.88 & 9.61 \\
\hline $\mathbf{0 . 7 8 6 8} \mathbf{~ m m o l} / \mathbf{L}$ & $\mathrm{O}_{3}$ gas lead in & & & & & & & \\
$\mathrm{Cu}^{2+}+\mathbf{A q}$. & Time $(\mathrm{min})$ & 0 & 1 & 3 & 6 & 10 & 14 & \\
\cline { 2 - 9 } Ozonation & $\mathrm{mg} / \mathrm{L} \mathrm{O}_{3}$ & 0.00 & 8.51 & 8.48 & 8.93 & 9.07 & 9.12 \\
\hline
\end{tabular}

In accordance with J. Carell Morris’ 1988 study, the solubility c* $\left(\mathrm{O}_{3}\right)_{\mathrm{a}}$ of ozone versus temperature $(\mathrm{T})$ is characterized by the following equation, when the temperature decreased, the ozone solubility increased:

$\log _{10} c^{*}\left(O_{3}\right)_{a}=-0.25-0.013 T$

Thus the dissolved ozone concentration should be higher at $0^{\circ} \mathrm{C}$ than at room temperature, as is demonstrated in Table 4.2. The copper catalytic ozonation at room temperature and at $0^{\circ} \mathrm{C}$ was conducted and the results showed that the concentration of ozone in water was higher at $0^{\circ} \mathrm{C}$ than at room temperature. While peroxide generation was much lower at $0^{\circ} \mathrm{C}$ than at room temperature, the higher ozone concentration led to the lower peroxide generation at $0^{\circ} \mathrm{C}$. The explanation is that peroxide generation is a heat absorption reaction, and the temperature effect was more significant than that of dissolved ozone concentration. It was also reported by Dasgupta et al. (1990) that peroxide generation was higher at $60^{\circ} \mathrm{C}$ than at room temperature during polypropylene slurry ozonation. The higher the temperature is, the faster the reaction. 
Table 4.2 Dissolved ozone concentration of $\mathrm{Cu}^{2+}$ catalytic ozonation at different temperatures (room temperature versus $0{ }^{\circ} \mathrm{C}$ )

\begin{tabular}{|c|c|c|c|c|c|c|c|}
\hline $\begin{array}{l}0.7868 \mathrm{mmol} / \mathrm{L} \\
\mathrm{Cu}^{2+}+\mathrm{Aq}\end{array}$ & $\begin{array}{c}\mathrm{O}_{3} \text { gas lead in } \\
\text { Time (min) }\end{array}$ & 0 & 1 & 3 & 6 & 10 & 14 \\
\hline Ozonation at $22^{\circ} \mathrm{C}$ & mg/L O3 & 0.00 & 8.51 & 8.48 & 8.93 & 9.07 & 9.12 \\
\hline $\begin{array}{l}0.7868 \mathrm{mmol} / \mathrm{L} \\
\mathrm{Cu}^{2+}+\mathrm{Aq} .\end{array}$ & $\begin{array}{l}\mathrm{O}_{3} \text { gas lead in } \\
\text { Time (min) }\end{array}$ & Blank & 1 & 3 & 5 & 15 & 30 \\
\hline Ozonation at $0^{\circ} \mathrm{C}$ & $\mathrm{mg} / \mathrm{L} \mathrm{O} 3$ & 0 & 7.77 & 13.34 & 14.77 & 13.66 & 13.77 \\
\hline
\end{tabular}

The ozone solution (at $25 \mathrm{~min}$ and $35 \mathrm{~min}$ ozonation time, respectively) at $0^{\circ} \mathrm{C}$ was scanned by UV from wavelength $200 \mathrm{~nm}$ to $320 \mathrm{~nm}$. The results showed that the dissolved ozone concentration had no change with or without $\mathrm{Cu}^{2+}$ catalysis. As shown in Figures 4.20 and 4.21 of the UV scan charts, both ozone peaks with or without copper catalyst, which occurred at $258 \mathrm{~nm}$, were the same. The peaks from $200 \mathrm{~nm}$ to $250 \mathrm{~nm}$ varied considerably, and this indicated that the intermediates in the aqueous phase ozonation with or without copper catalyst were different.

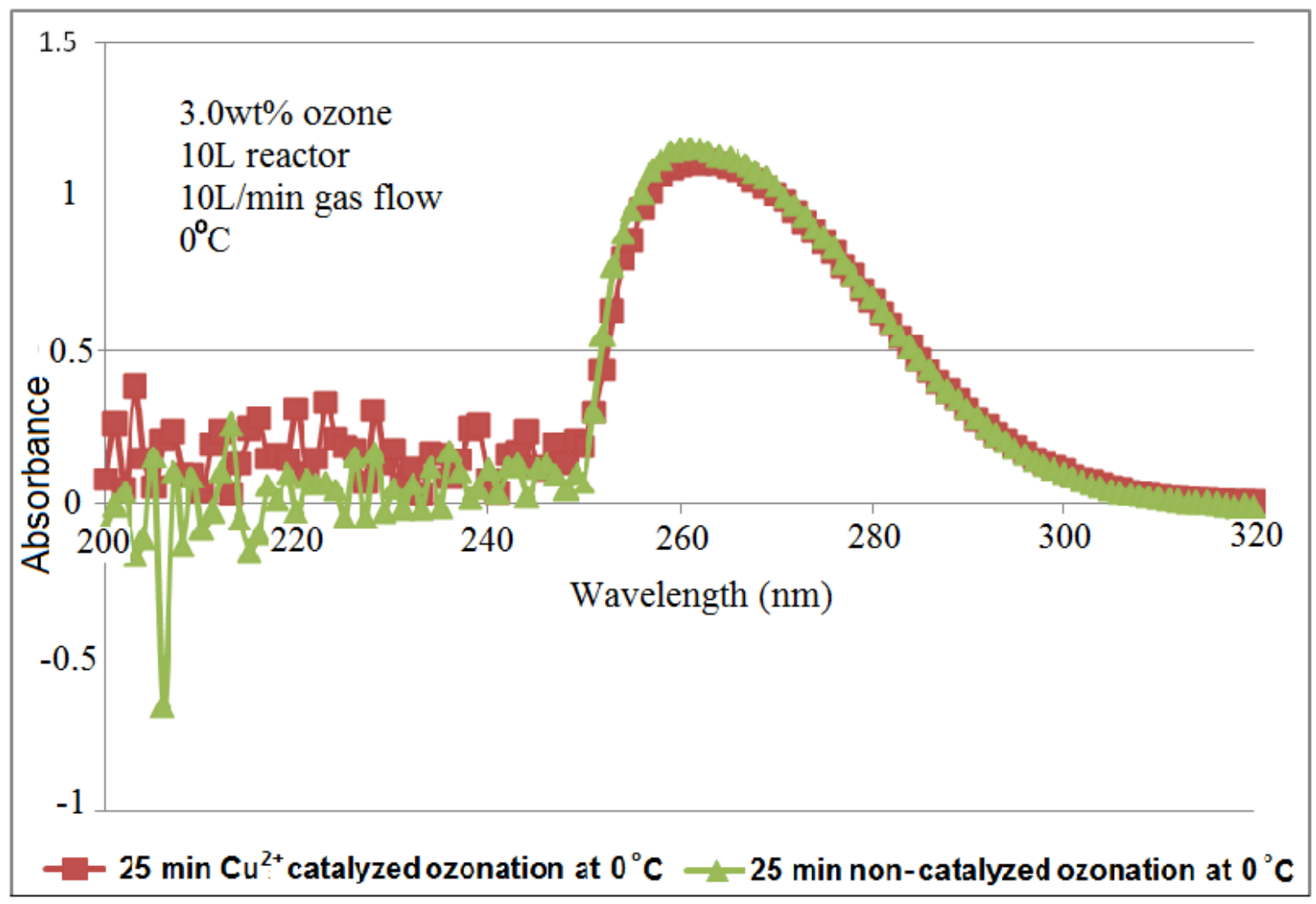

Fig 4.20 UV scan chart for $25 \mathrm{~min}$ of aqueous ozonation at $0^{\circ} \mathrm{C}$ with/without catalyst 


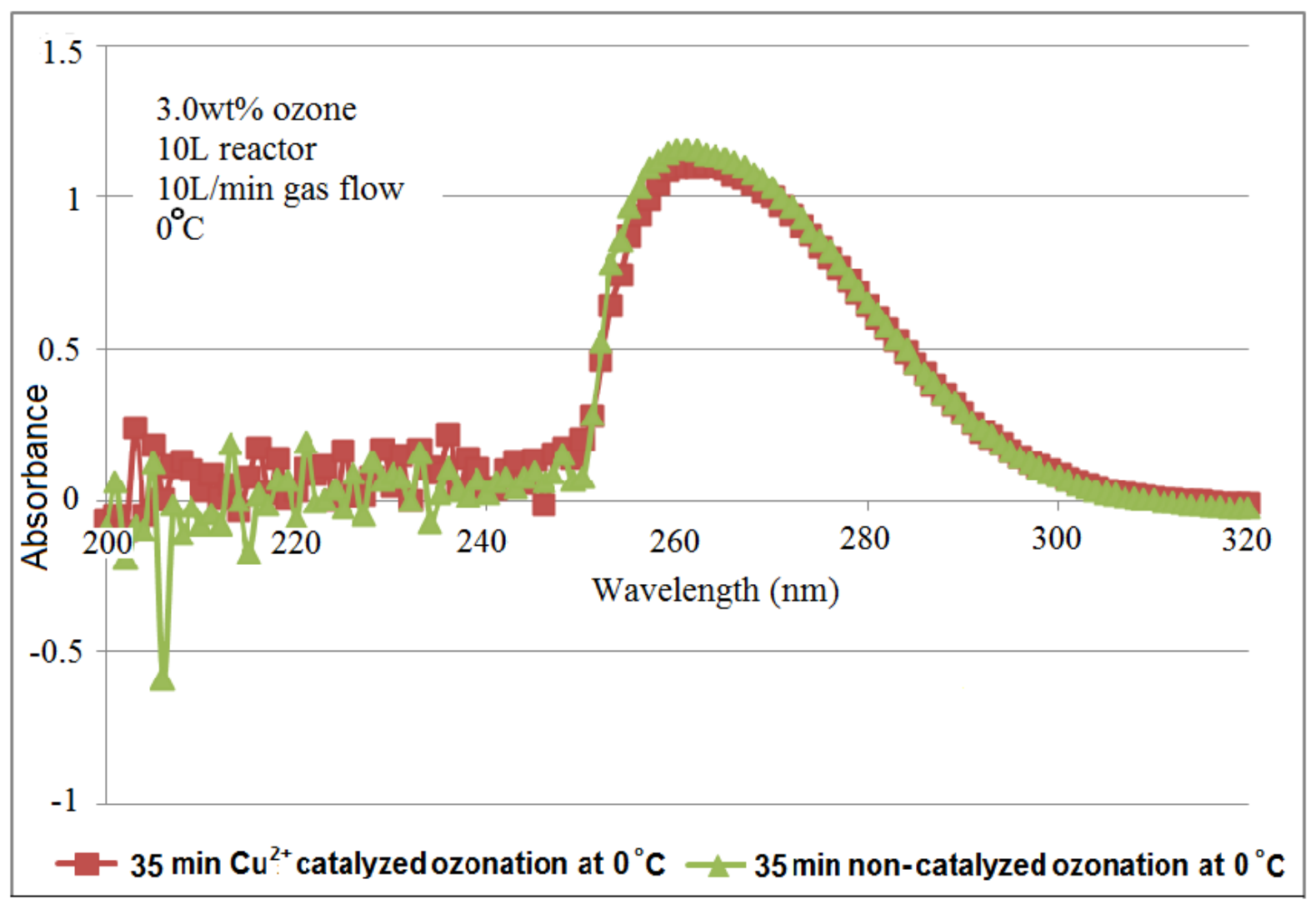

Fig 4.21 UV scan chart for $35 \mathrm{~min}$ of aqueous ozonation at $0^{\circ} \mathrm{C}$ with/without catalyst

4.1.8.5 Hydroxyl radical capture by Nitrobenzene applied to ozonation with or without $\mathrm{Cu}^{2+}$ catalyst

In Section 4.1.8.1, the scavengers' effect on ozonation with/without $\mathrm{Cu}^{2+}$ catalyst was investigated, and the radicals were found to be the factor in the catalytic ozonation that increased the peroxide generation. Generally, hydroxyl radicals were assumed to be those generated as intermediates for ozone reaction with organic chemicals in waste water (Beltran, 2004). A method of special radical capture is necessary to further confirm the existence of the radicals for ozonation of PPMM. Nitrobenzene (NB) is a chemical for capturing the hydroxyl radicals. The author referred to Feng et al. 2011's "Experimental Determination of Hydroxyl Radical Reactivity in Supercritical Water Using Pulse Radiolysis," and applied $25 \mathrm{ml}$ NB (99\% purity) to $75 \mathrm{ml}$ ozonation solution at different ozonation times to capture the hydroxyl radicals by forcefully shaking the flasks for 5 minutes. NB and water were separated after the capture procedure, and the samples of reacted NB were removed and tested using UV-Vis at the single wavelength of $410 \mathrm{~nm}$. The addition of hydroxyl radicals to nitrobenzene forms the 
easily detected radical Nitrohydroxycyclohexadienyl - • $\mathrm{OHC}_{6} \mathrm{H}_{5} \mathrm{NO}_{2}$. It is detectable because of the increase in the absorbance of the product (NB itself has a light brown color, so the UV-Vis absorption was not zero at 0 min ozonation). The chemical reaction is shown in Equation (4.2) (Feng et al., 2011).

$\mathrm{C}_{6} \mathrm{H}_{5} \mathrm{NO}_{2}+\cdot \mathrm{OH} \rightarrow \cdot \mathrm{OHC}_{6} \mathrm{H}_{5} \mathrm{NO}_{2}$

The UV-Vis testing results are shown in Figure 4.22 and Figure 4.23. The results indicated that the $\cdot \mathrm{OH}$ was present in the distilled water and the copper (II) catalytic ozonation solution and was captured by the nitrobenzene, because the absorption increased as compared to that of the blank liquid. Comparison of Figure 4.22 and 4.23 shows that catalytic ozonation did generated more hydroxyl radicals.

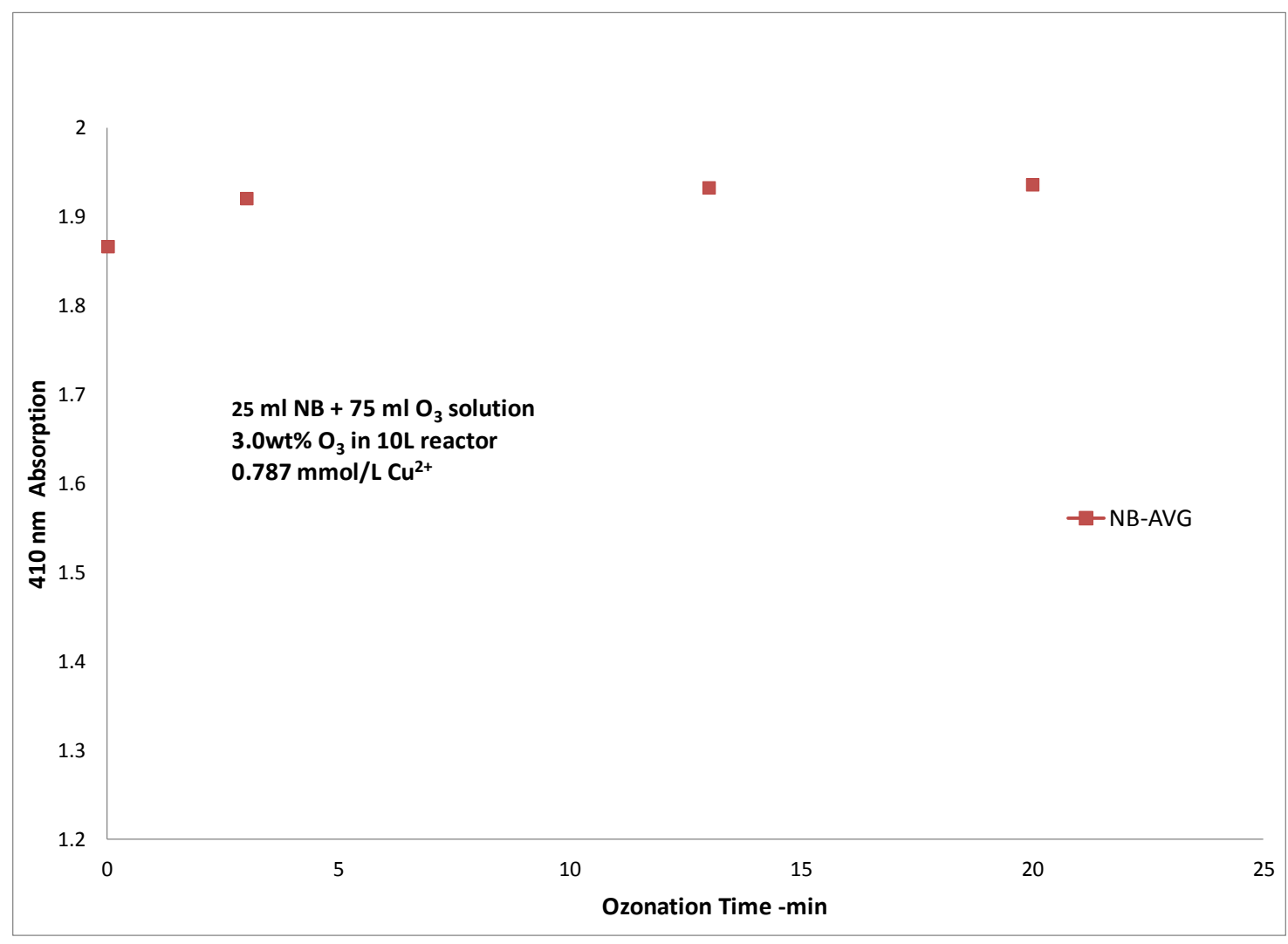

Fig 4.22 Detection of $\mathrm{NB}$ captured $\cdot \mathrm{OH}$ in the $\mathrm{Cu}^{2+}$ catalyzed ozonation 


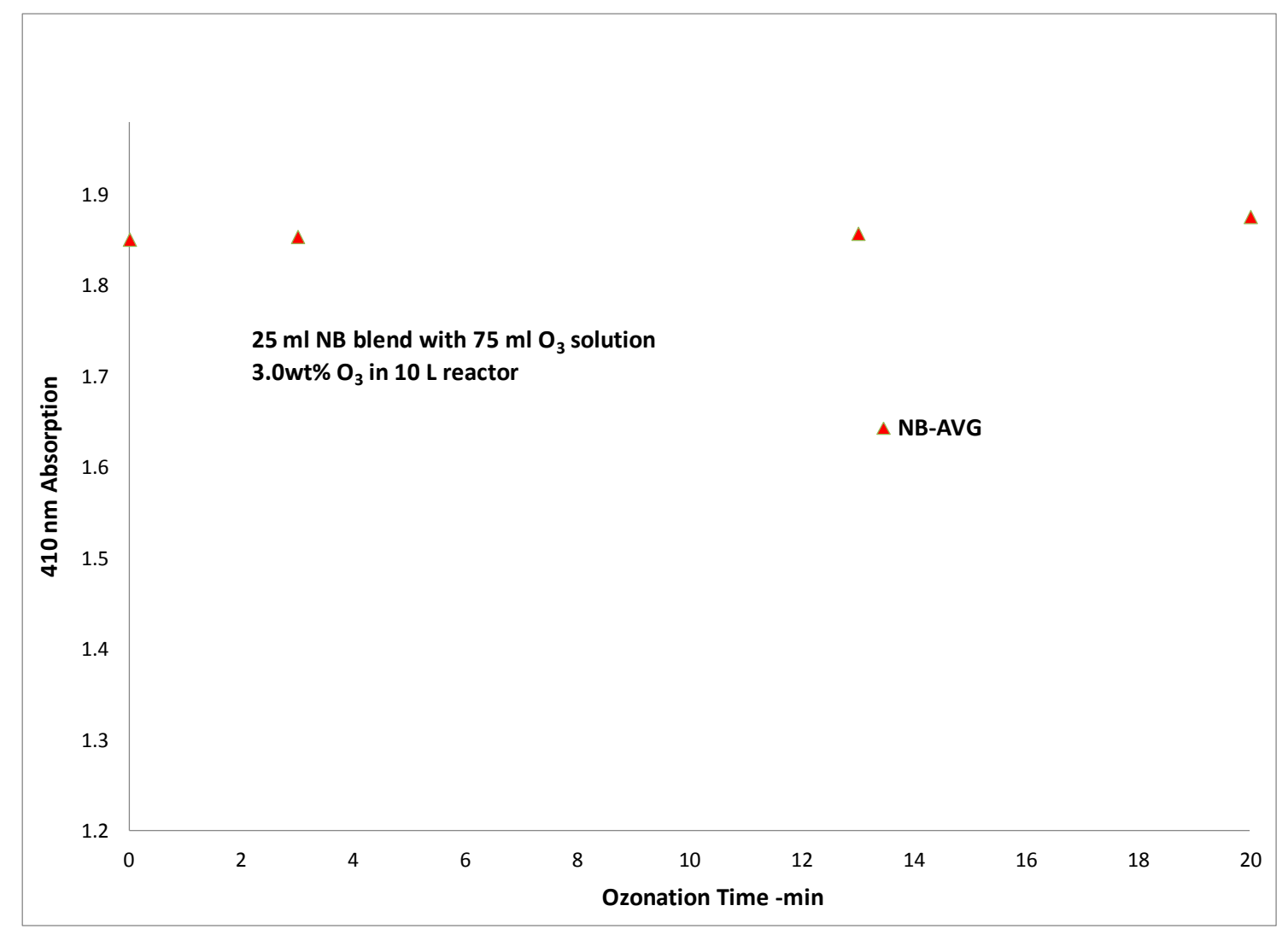

Fig 4.23 Detection of NB captured $\cdot \mathrm{OH}$ in non-catalyzed ozonation

\subsubsection{Homogeneous catalyst test using $\mathrm{Fe}^{3+}$}

Another metal ion, $\mathrm{Fe}^{3+}$ (ferric sulfate), also can be used as a homogeneous catalyst and can accelerate the peroxide generation on polyethylene film (Pastel et al., 2009). What would the result be if $\mathrm{Fe}^{3+}$ was applied to PPMM? In these experiments, $\mathrm{Fe}^{3+}$ catalyst was applied to PPMM, as shown in Figure 4.24. Specifically, $0.0616 \mathrm{mmol} / \mathrm{L}, 0.3696 \mathrm{mmol} / \mathrm{L}$, $0.6160 \mathrm{mmol} / \mathrm{L}$, and $3.6959 \mathrm{mmol} / \mathrm{L}$ of $\mathrm{Fe}^{3+}$ were added to the aqueous phase ozonation at $3.0 \mathrm{wt} \% \mathrm{O}_{3}$ for 30 minutes. It was found that both $0.3696 \mathrm{mmol} / \mathrm{L}$ and $0.6160 \mathrm{mmol} / \mathrm{L}$ of $\mathrm{Fe}^{3+}$ made satisfatory contribution to the peroxide generation. $3.6959 \mathrm{mmol} / \mathrm{L} \mathrm{Fe}^{3+}$ accelerated the peroxide generation rate, but the increase was smaller than that of $0.3696 \& 0.6160 \mathrm{mmol} / \mathrm{L}$. The high concentration of $\mathrm{Fe}^{3+}(3.6959 \mathrm{mmol} / \mathrm{L})$ had a decreasing effect on the peroxide generation rate due to the high concentration of $\mathrm{SO}_{4}{ }^{2-}$ anions. $\mathrm{pH}$ value was measured from 3.8 to 2.2 when $\mathrm{Fe}^{3+}$ concentrate varied from $0.0616 \mathrm{mmol} / \mathrm{L}$ to $3.6959 \mathrm{mmol} / \mathrm{L}$. From economic point of view, $0.3696 \mathrm{mmol} / \mathrm{L}$ of $\mathrm{Fe}^{3+}$ should be selected for practical application. 


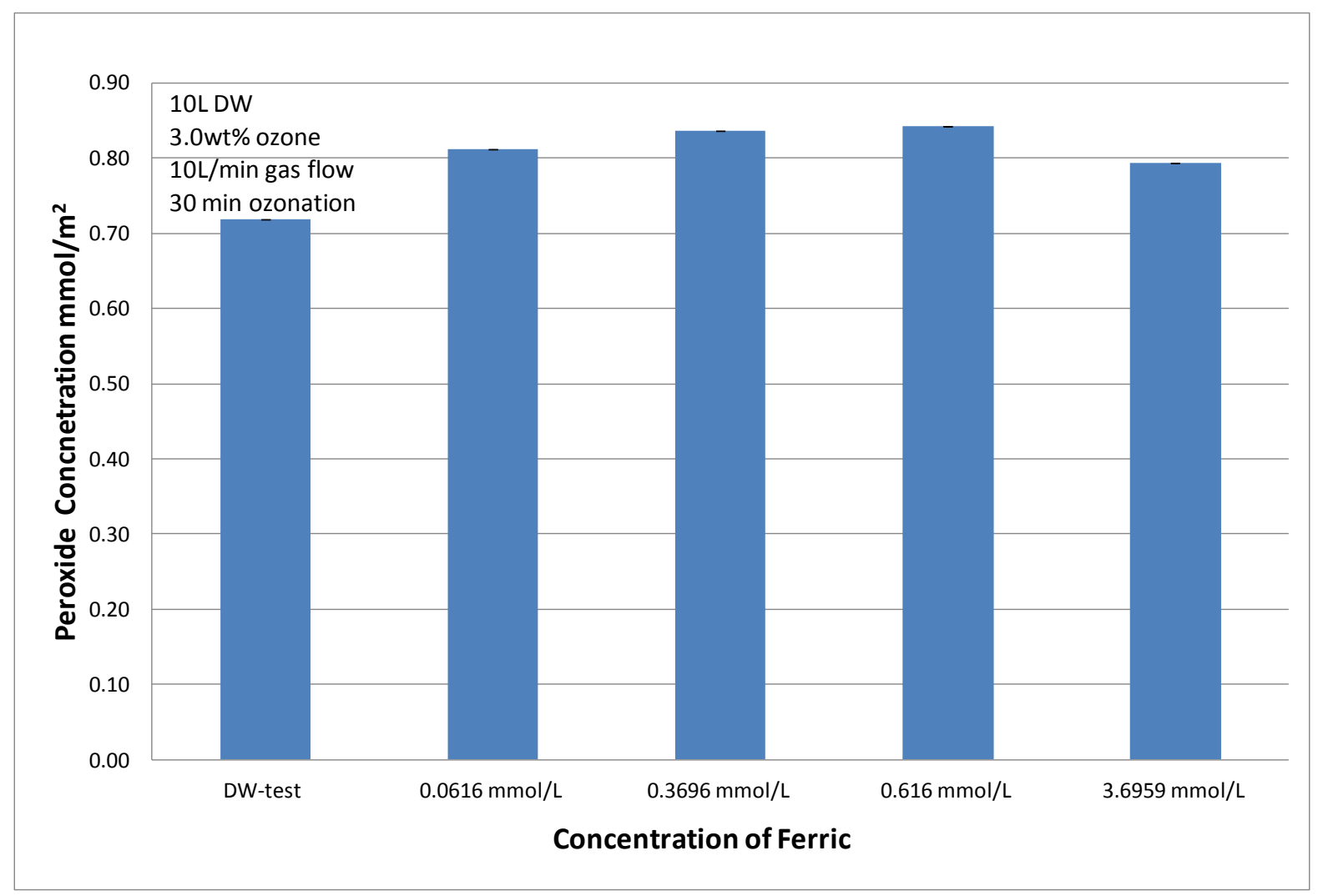

Fig 4.24 Catalytic ozonation of PPMM using $\mathrm{Fe}^{3+}$ catalyst

\subsection{Polypropylene Film Ozonation}

Biaxial oriented polypropylene (BOPP) film makes excellent packaging and medical materials. However, it is highly hydrophobic. The hydrophobic property, when it is not modified, limits its use in packaging applications that have low bonding strength to laminate with other films, and in medical applications. There are many methods that are applied to improve its hydrophilicity, and ozonation is one method that has been studied in this research.

The surface area of BOPP is much smaller than that of PPMM because there are no pores, it needs to be explored whether the effect of ozonation is the same or not. The peroxide concentration of BOPP ozonation was detected much less than that of PPMM, but there are many similar characteristics shared by BOPP and PPMM, which will be discussed in the following sections.

\subsubsection{Ozonation of BOPP in the aqueous and gaseous phases}

Referring to my M.A.Sc thesis (Gu, H., 2008), BOPP films were ozonated in the 
aqueous (distilled water) and in the gaseous phase, respectively, to compare the efficiency of ozonation in different reaction media. The ozonation conditions were as follows: ozone concentration $3.0 \mathrm{wt} \%$, gas flow rate $10.0 \mathrm{~L} / \mathrm{min}$, pressure $103.4 \mathrm{kPa}$ (15 psig), room temperature $22^{\circ} \mathrm{C}$. Figure 4.25 shows the results. Peroxide generation increased with ozonation time regardless of whether the ozonation was conducted in the aqueous or the gaseous phase. When the same ozone dose was applied, the detected peroxide concentration generated by aqueous phase ozonation was a little lower than that generated by gaseous phase ozonation.

\subsubsection{Washing test}

A washing test was applied to the ozonated BOPP films immediately after degasing. To wash the BOPP that had been ozonated in the gaseous phase and the aqueous phase, $10 \%$ (v/v) IPA (used to increase the wetting of the BOPP in DW) in distilled water was employed for 15 minutes respectively. The peroxide concentration was analyzed and compared with that of the BOPP that had not undergone the washing test. It was found that the peroxide concentration of the BOPP that undergone the gaseous phase ozonation decreased to a lower level after washing than that of its aqueous counterpart which had been treated with the same ozone dose and for the same treatment time, as shown in Figure 4.26 and 4.27. This outcome was the same as for the ozonated PPMM. Ozonation broke the polypropylene chains and generated small oxidized molecules with peroxide moieties. However, the small oxidized molecules with peroxide moieties made no contribution to the graft polymerization and dissolved or were removed either by the distilled water in the aqueous phase ozonation or in the AAm IPA solution during the graft polymerization, or they were removed during the cleaning procedure. The results of this test supported the supposition that the aqueous phase ozonation could initiate more AAm graft polymerization than the gaseous phase ozonation, because the peroxide on the large polymer molecules was retained on the membranes and was effective in grafting monomers, even though the detected peroxide concentration was lower. 


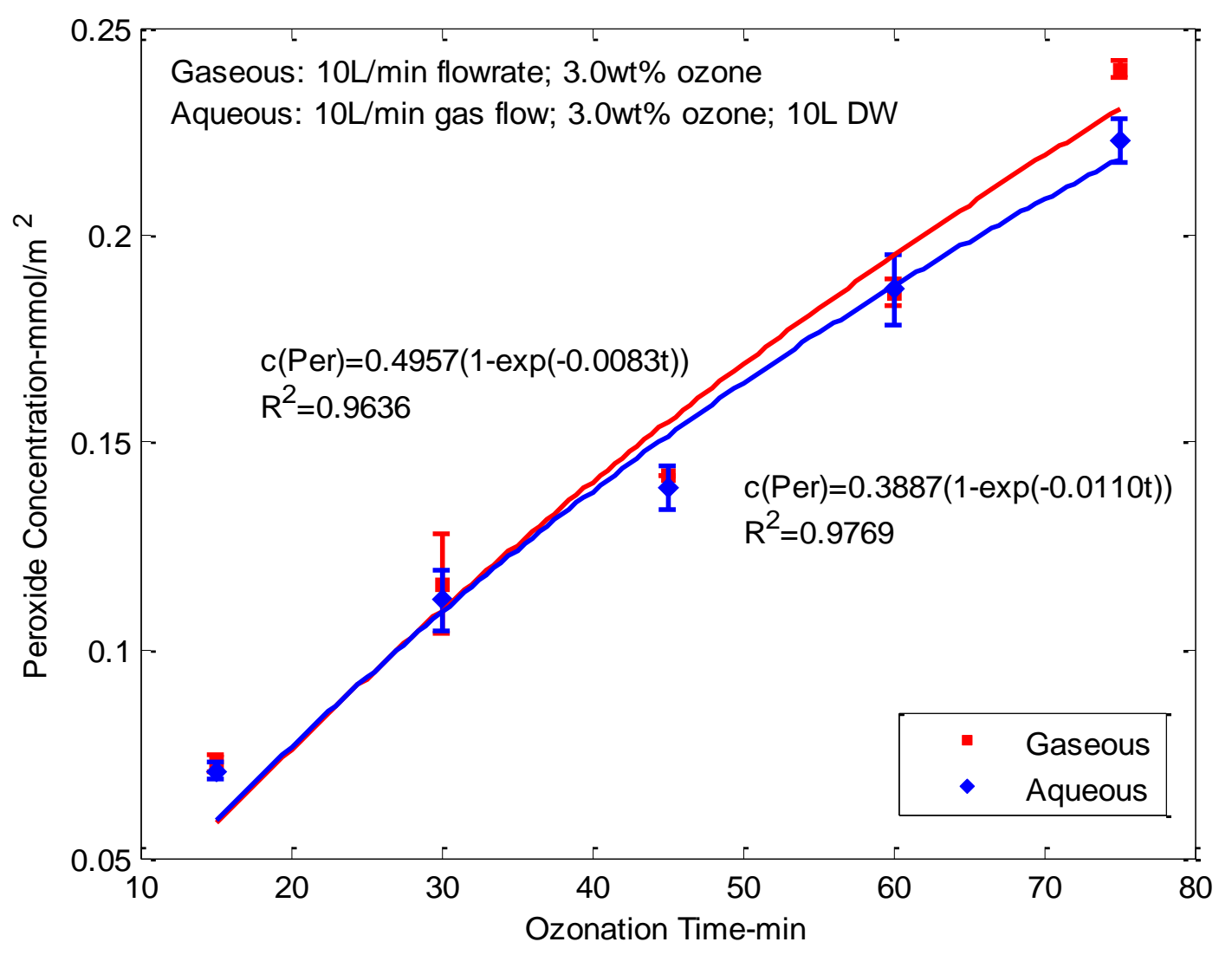

Fig 4.25 Peroxide generation in the ozonation of BOPP in the aqueous and gaseous phases

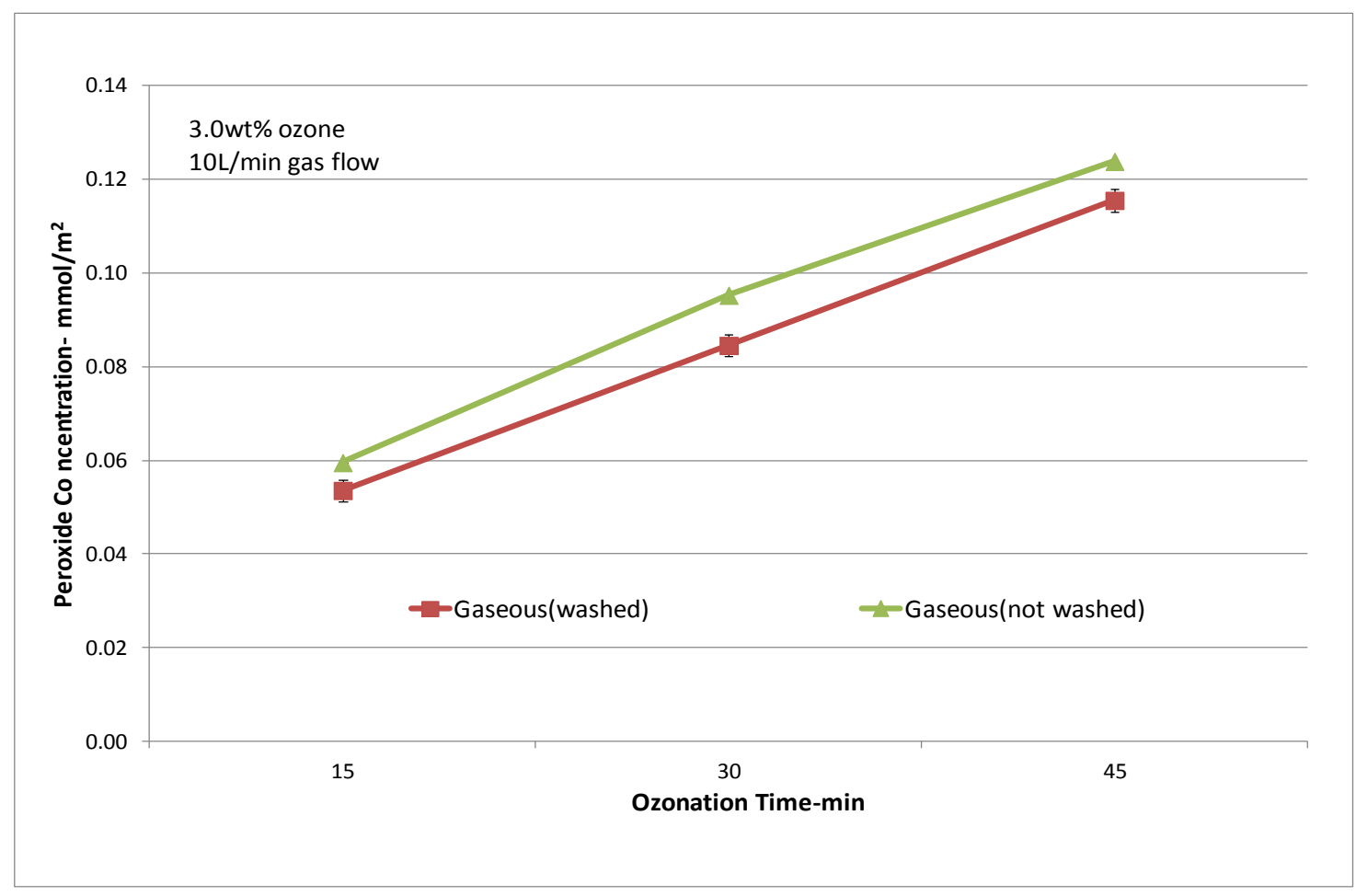

Fig 4.26 Comparison of peroxide concentration on BOPP treated by gaseous phase ozonation 
with or without washing

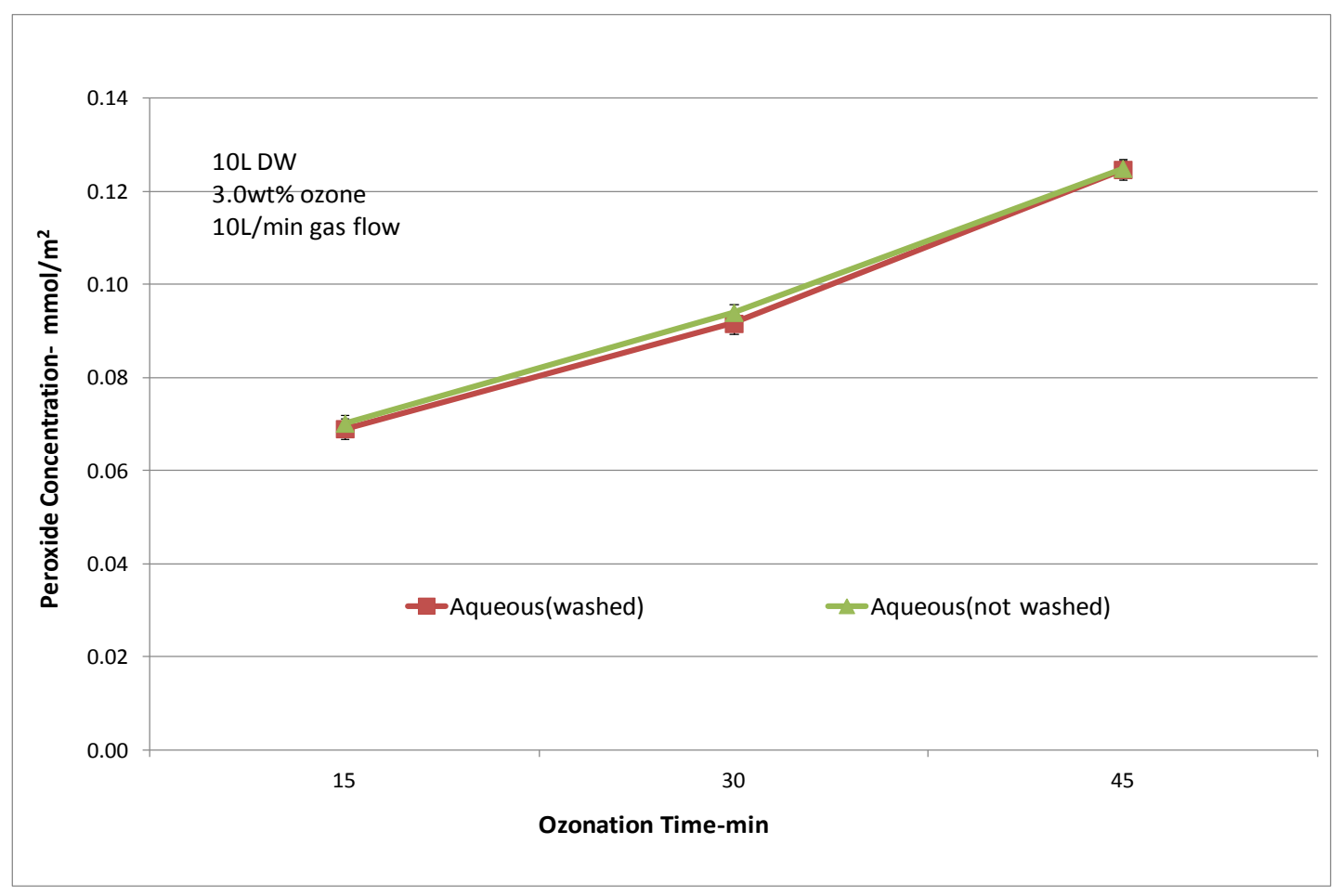

Fig 4.27 Comparison of peroxide concentration on BOPP treated by aqueous phase ozonation with or without washing

\subsubsection{Peroxide aging test of BOPP}

To study the peroxide stability on the BOPP film, ozonated BOPP films were tested after 1 day, 3 days, 14 days, and 28 days using the iodometric method, and the results are shown in Figure 4.28. The peroxide concentration decreased slowly from 0-3 days; it maintained $95.7 \%$ activity after three days, and still kept about $60 \%$ activity after 4 weeks. The results indicate that active points were fairly stable for graft polymerization. 


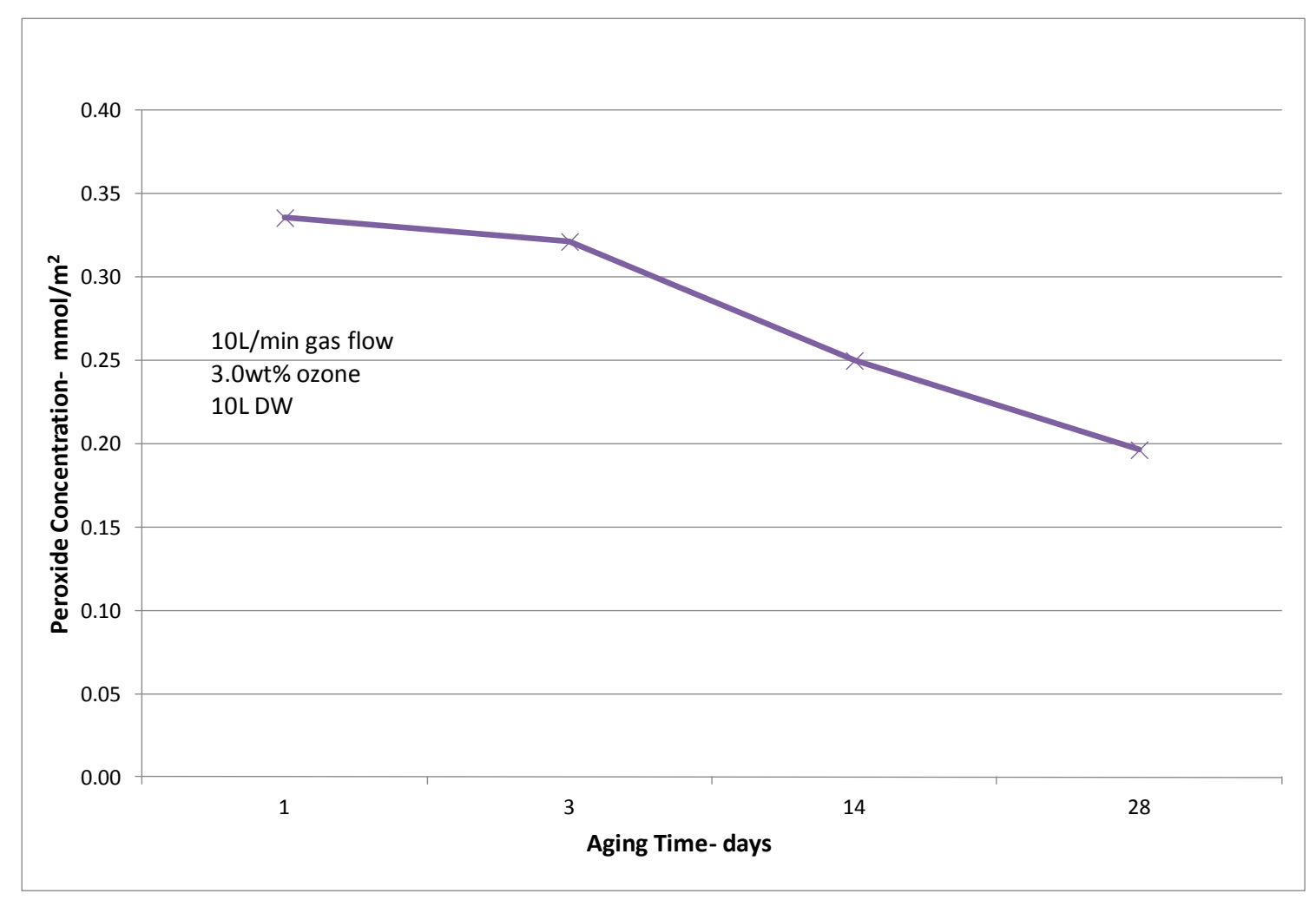

Fig 4.28 Results of the aging test of peroxides generated from aqueous ozonation of BOPP

\subsubsection{Scavenger test}

A radical scavenger test was conducted on the aqueous phase ozonation applied to BOPP film. The results of the tests are shown in Figure 4.29. To eliminate the free radicals, $3.0 \mathrm{~g} / \mathrm{L}$ sodium carbonate was added to the distilled water. It was found that the generated peroxide concentration was much lower when the scavenger was added. The results indicate that the maximum contribution to the peroxide generation made by the free radicals in this test was $24 \%\left(0.0952 \mathrm{mmol} / \mathrm{m}^{2}\right.$ vs. $0.0726 \mathrm{mmol} / \mathrm{m}^{2}$ at $30 \mathrm{~min}$ ozonation). 


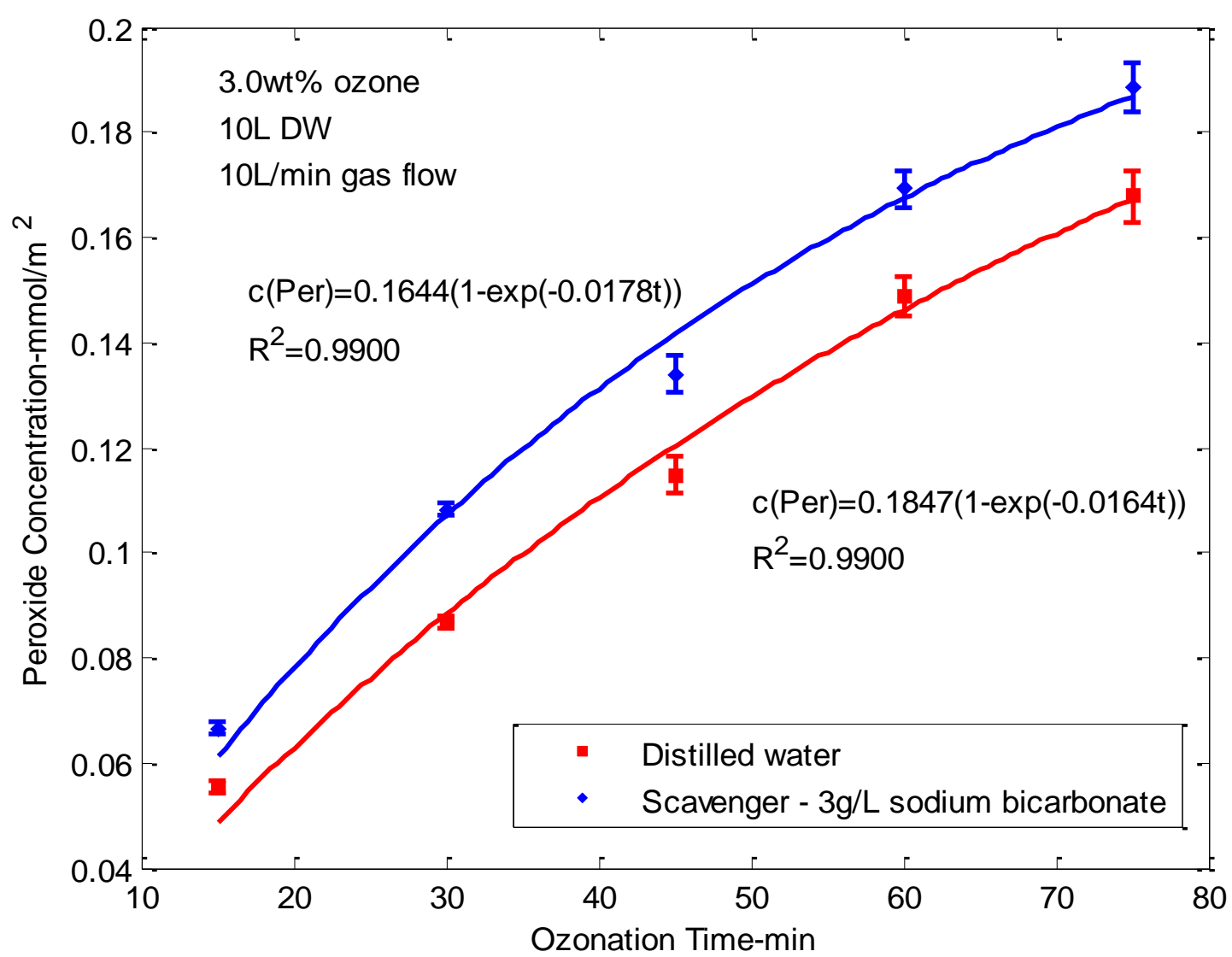

Fig 4.29 Peroxide generation from aqueous ozonation of BOPP with and without $3.0 \mathrm{~g} / \mathrm{L}$ of sodium carbonate as a scavenger

\subsection{5 pH test}

Ozonation of BOPP film at different $\mathrm{pH}$ levels was investigated using the same ozone dose (3.0 wt $\%)$ and reaction time (30 minutes), and the peroxide concentration was found to decrease when $\mathrm{pH}$ value increased, as shown in Figure 4.30. The reaction kinetics is the combination of reactions with gas ozone, dissolved molecular ozone and radicals, and was affected by their concentration, diffusion and life time as discussed previously in Section 4.1.6 for $\mathrm{pH}$ test of PPMM ozonation. 


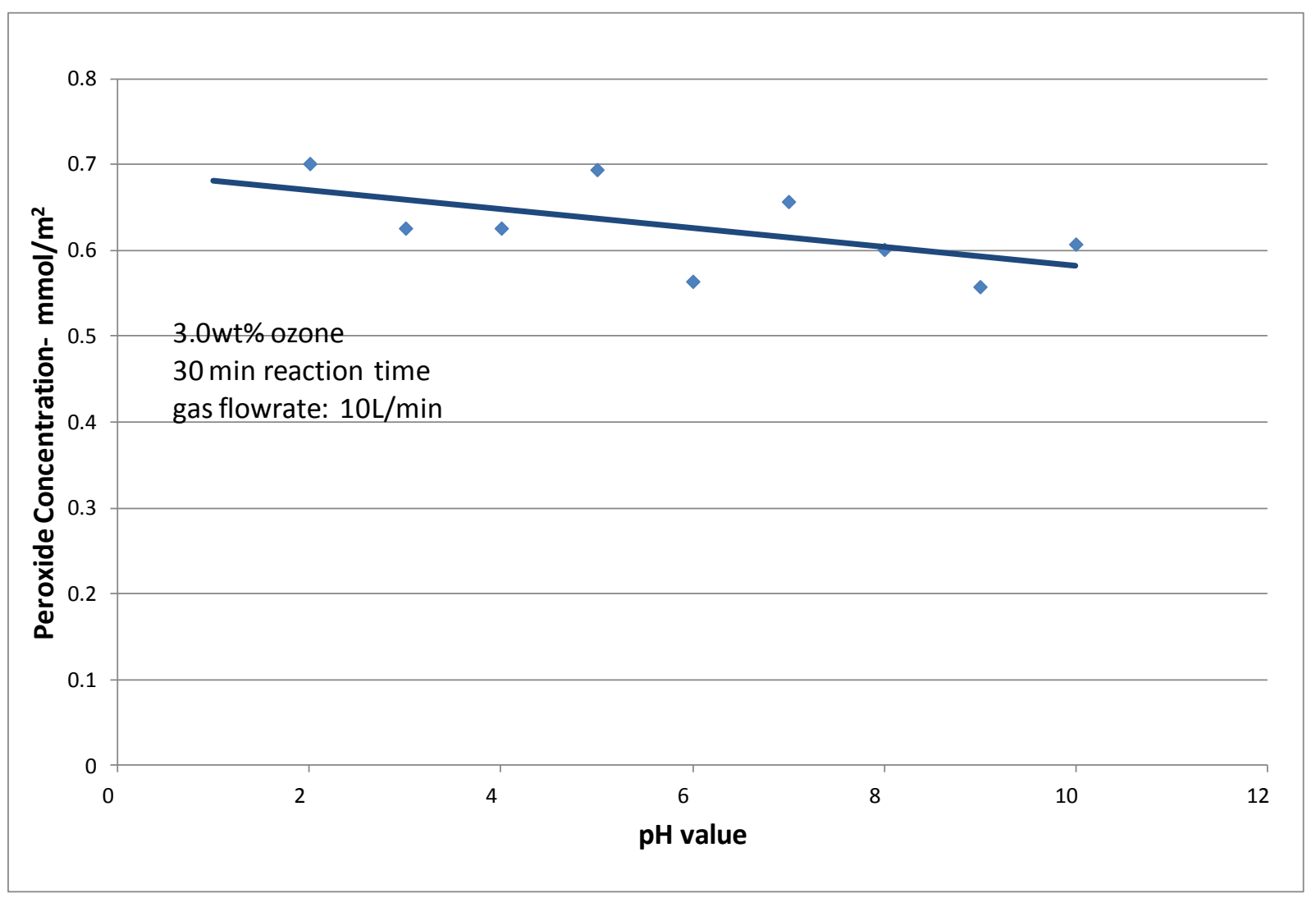

Fig 4.30 Peroxide generation of BOPP at different $\mathrm{pH}$ levels

\subsubsection{Homogeneous catalyst test $-\mathrm{Fe}^{3+}$}

As shown in Figure 4.31, aqueous phase ozonation of BOPP films was conducted with and without $\mathrm{Fe}^{3+}$ catalyst, and it was found that more peroxide was generated by the catalytic ozonation (for a maximum $16 \%$ increase compared to the peroxide level generated by ozonation in $\mathrm{DW}-0.116 \mathrm{mmol} / \mathrm{m}^{2}$ vs. $0.099 \mathrm{mmol} / \mathrm{m}^{2}$ at 30 minutes). The mechanism is discussed in Section 4.6.3.2. 


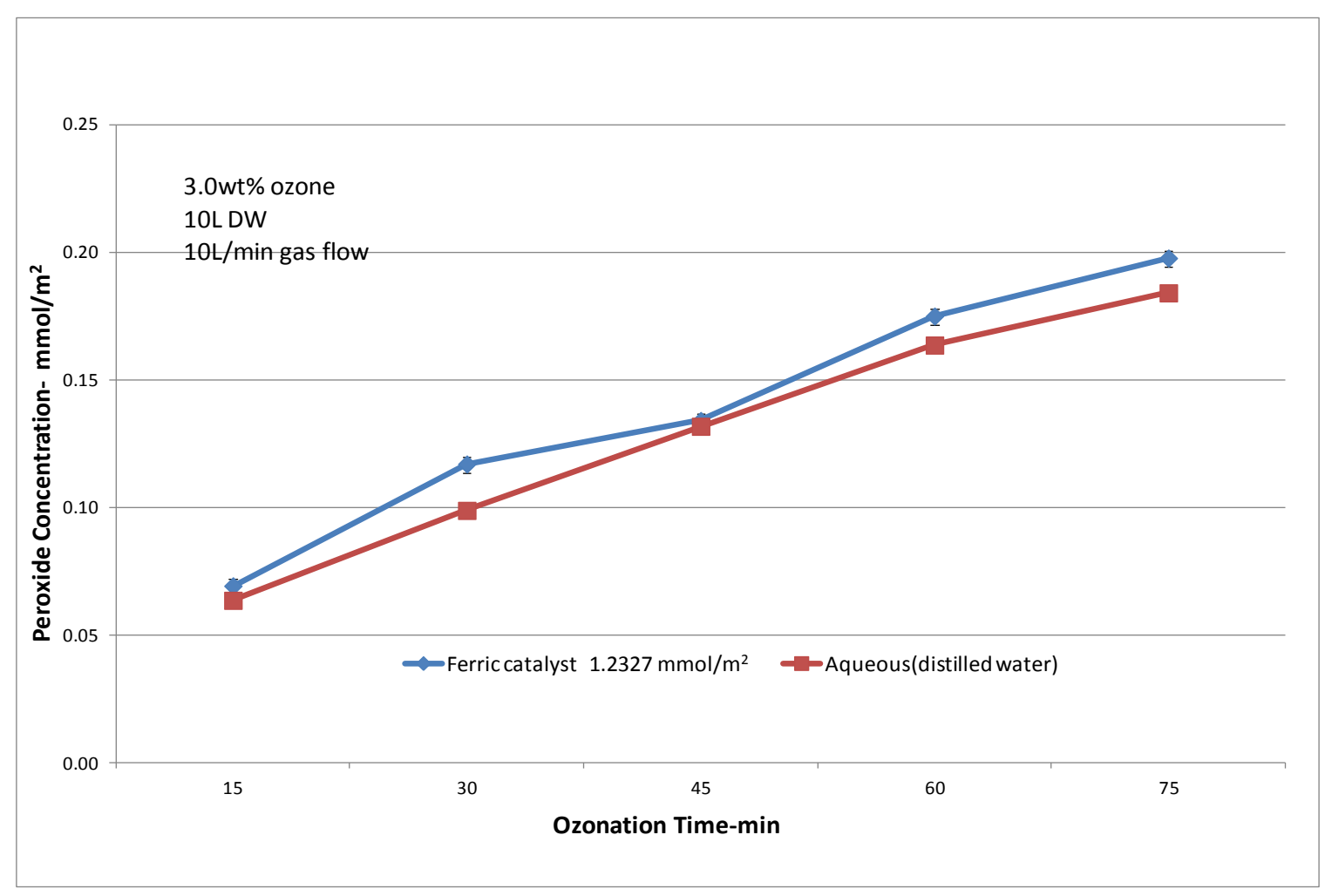

Fig 4.31 Effect of different ozonation times for $\mathrm{Fe}^{3+}$ catalyzed ozonation of BOPP

\subsection{Graft Polymerization}

Although peroxides have been generated, their accessibility to monomers still needs to be evaluated. Therefore, graft polymerization was conducted in the following sections; the results were induced by aqueous and gaseous phase ozonation and compared. The samples were employed a cleaning procedure after graft polymerization with DW and acetone in the incubator shaker, the homopolymers were removed.

\subsubsection{Graft polymerization and FTIR analysis}

Graft polymerization of different monomers induced by peroxides was investigated, dried samples were analyzed by FTIR, and the results were compared.

\subsubsection{Acrylic amide (AAm) grafted to PPMM and FTIR analysis}

Ozonated membranes were dried and degassed under vacuum for 2 hours, then immersed in the $\mathrm{N}_{2}$ purged and degassed 20\% AAm IPA solution (Yuan et al., 2003), the graft polymerization was conducted at $80^{\circ} \mathrm{C}$ and $120 \mathrm{rpm}$ in a water bath for 20 hours (different 
grafting time was tested, 20 hours was found a suitable time to obtain more graft polymerization instead of homo-polymerization). The washed and dried samples were sent for FTIR analysis. The functional groups on the film surfaces were then characterized by FTIR. Figures 4.32 and 4.33 show the FTIR spectra of the virgin and grafted membranes ozonated in aqueous and gaseous phases, and Figure 4.34 shows the comparison of the FTIR spectra after aqueous and gaseous ozonation and AAm grafting. After the AAm graft polymerization, a strong peak appeared at $1668 \mathrm{~cm}^{-1}$, and a weak, wide peak appeared at $3200-3400 \mathrm{~cm}^{-1}$. The peak at $1668 \mathrm{~cm}^{-1}$ is the characteristic frequency of the C-N amide group, and the peak at $3347 \mathrm{~cm}^{-1}$ is the characteristic frequency of $\mathrm{N}-\mathrm{H}$ (Wu et al., 2000; Yong et al., 2005; Yuan et al., 2003). According to Figure 4.34, it is evident that the characteristic peaks were larger for A15 + AAm $(\mathrm{A} 15=15$ minutes of aqueous phase ozonation, $A A m=$ acrylic amide graft $)$ than for $\mathrm{G} 15+\mathrm{AAm}(\mathrm{G} 15=15$ minutes of gaseous phase ozonation), which indicated that the degree of AAm grafting was greater for the membrane samples ozonated in aqueous phase than their counterparts ozonated in gaseous phase at the same ozone dose. The results shown in Figure 4.32 and 4.33 also demonstrate that the peroxides generated by ozonation were accessible to AAm monomers.

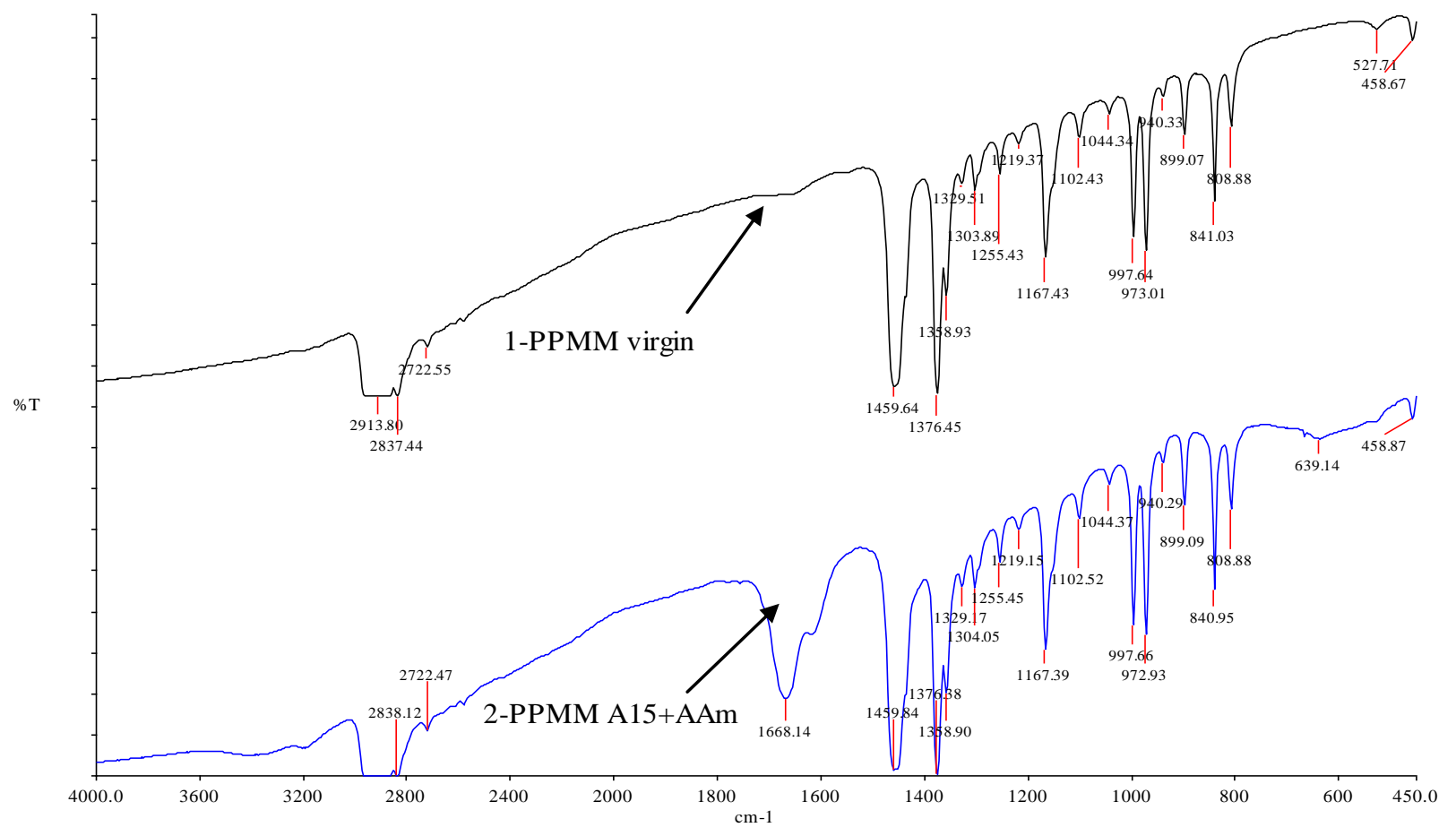

Fig 4.32 Results of FTIR analysis of PPMM after aqueous phase ozonation and AAm grafting 


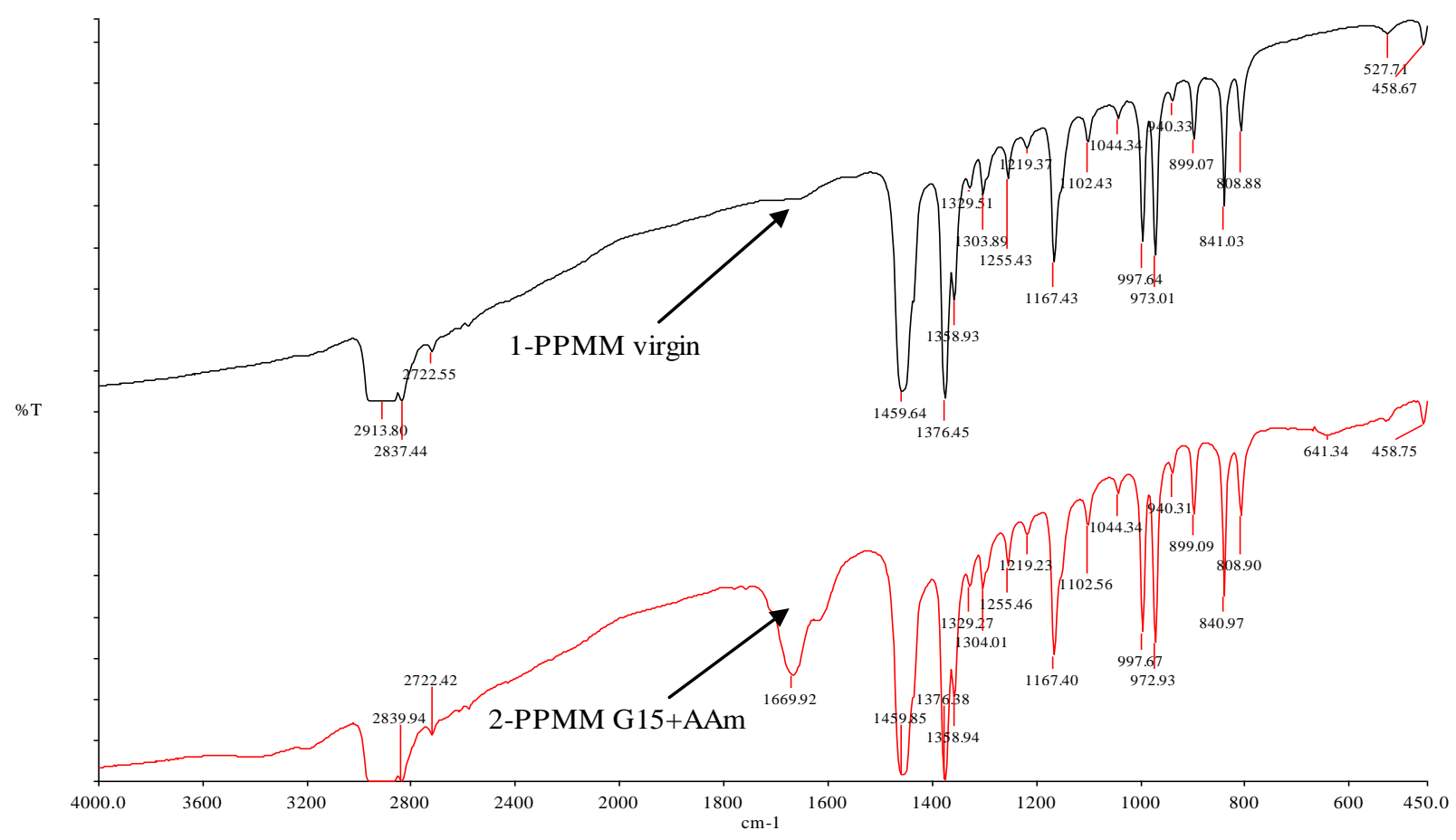

Fig 4.33 Results of FTIR analysis of PPMM after gaseous phase ozonation and AAm grafting

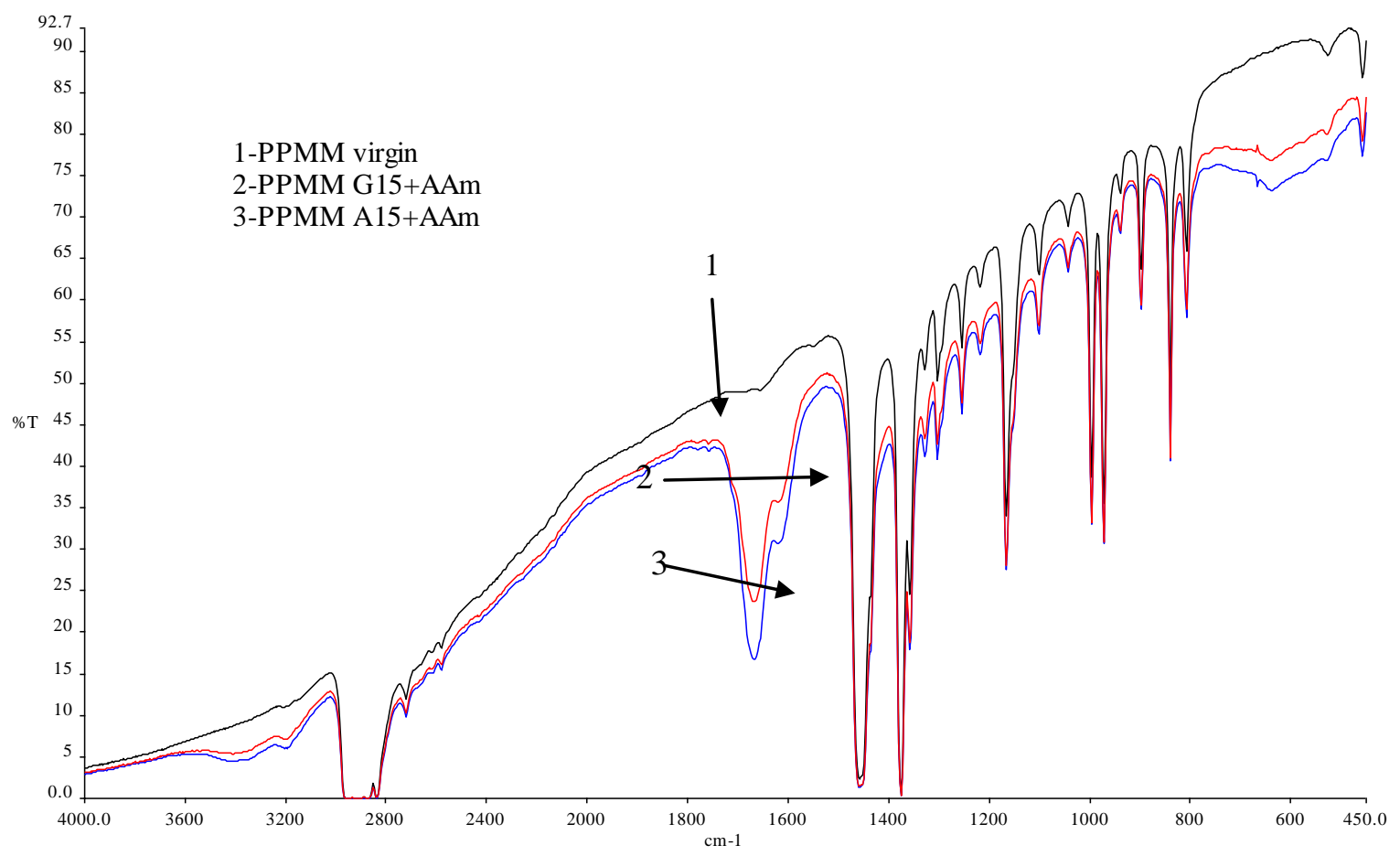

Fig 4.34 Comparison of results of FTIR analysis of PPMM after aqueous and gaseous phase ozonation and AAm grafting 


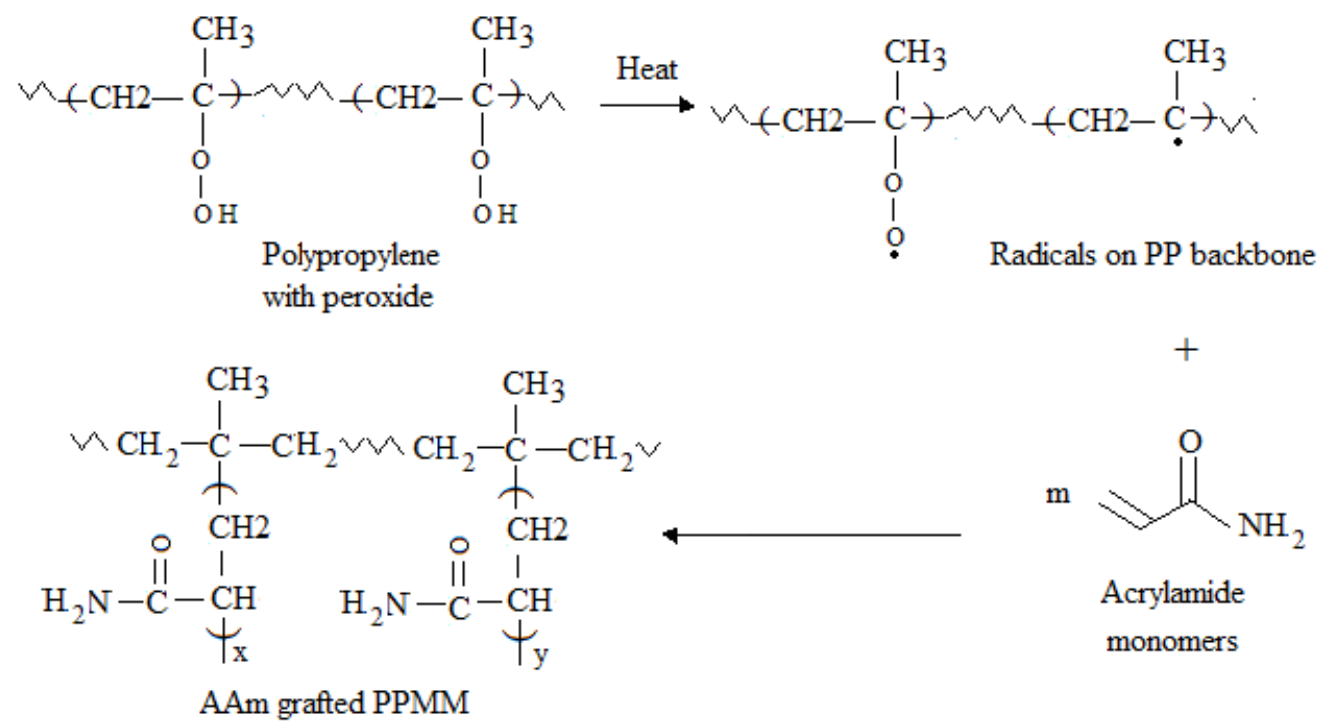

Fig 4.35 Chemical reaction schematic of AAm graft onto PPMM

The graft chemical reaction schematic in Figure 4.35 shows that the peroxide on PPMM backbone molecules decomposed to $\mathrm{R} \cdot$, or ROO • radicals (Razumovskii et al., 1971) (R-alkyl) when heated, the radicals induced the radical polymerization when they came into contact with the vinyl part of the AAm monomers; the double bond was broken by the radicals and further chain reaction occurred, and thus propagating the reaction. The reaction terminated when the radicals hit other radicals $\mathrm{R} \bullet, \mathrm{ROO} \cdot$ or $\mathrm{HO} \cdot$, and the radicals combined.

\subsubsection{Polyethylene glycol (PEG) grafted to PPMM and FTIR analysis}

Another monomer was also used to test the accessibility of peroxide sites. Ozonated and dried membranes were immersed in the $\mathrm{N}_{2}$ purged and degassed 20\% PEG200, PEG600, and PEG 3350 solution, respectively, where the numbers represented molecular weight of PEG; graft polymerization was conducted at $55^{\circ} \mathrm{C}$ and $120 \mathrm{rpm}$ in a water bath shaker, and the membranes were left for 20 hours. The washed and dried samples were sent for FTIR analysis, where the functional groups on the film surfaces were characterized. Figure 4.36 shows the FTIR spectra. A strong peak appeared at $1706 \mathrm{~cm}^{-1}(\mathrm{C}=\mathrm{O}$ group$)$, and a weak, wide peak appeared at $3200 \mathrm{~cm}^{-1}$ (O-H group) (Davidson et al., 2004; Mitchell et al., 2005) after graft polymerization using PEG. Figure 4.36, with the characteristic peaks, which were larger 
and deeper for the samples grafted by PEG200, showed that the monomer PEG200 was able to access the active points of the ozonated membranes easily; PEG600 was less able, and PEG3350 was the least able. These results indicate that the larger the monomer size of the PEG, the harder it was for it to access the reactive points. The mechanism of PEG graft polymerization was different from AAm graft polymerization, the hydroxyl groups of PEG could react with the generated carbonyl and carboxyl groups.

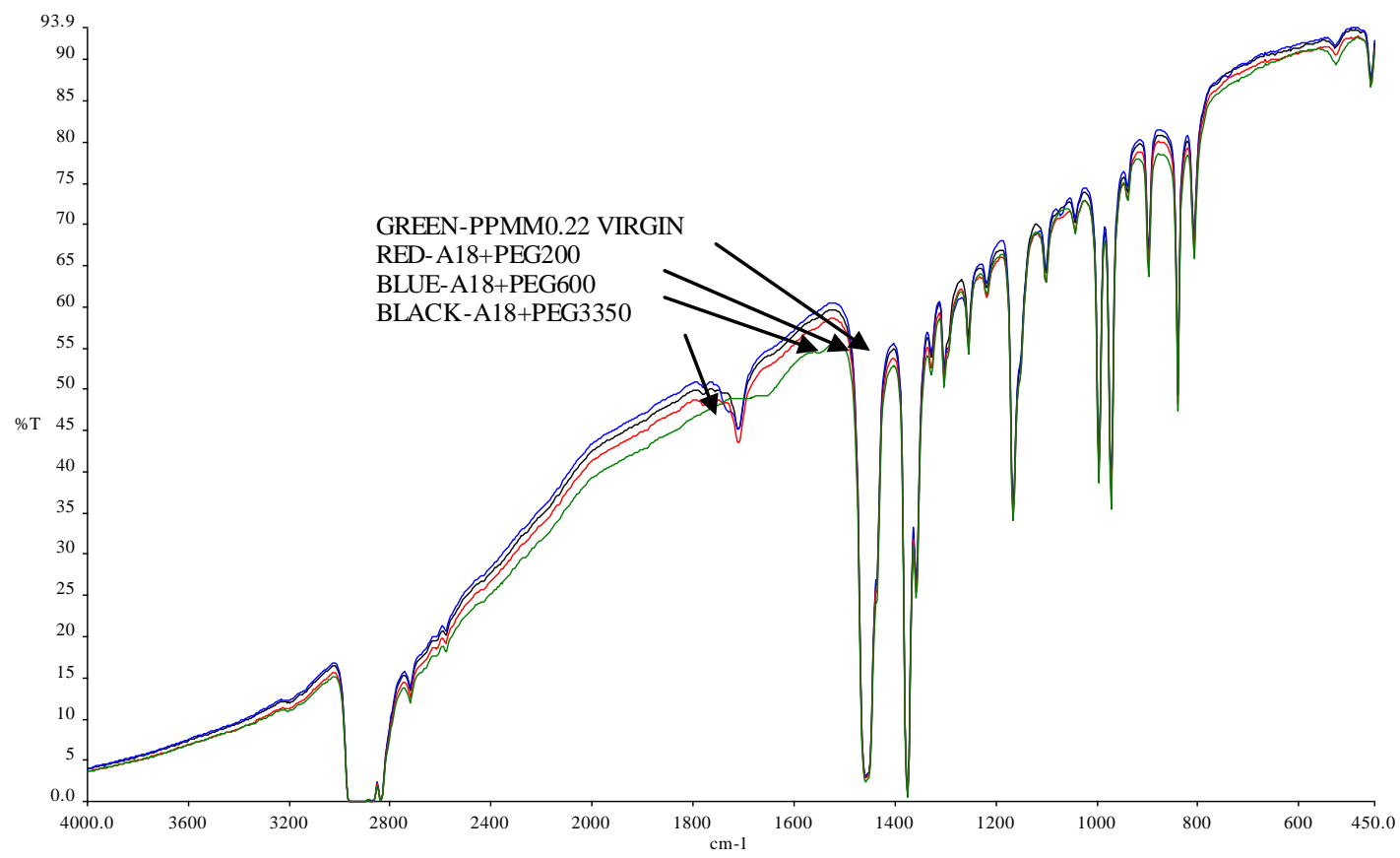

Fig 4.36 Comparison of FTIR results for aqueous phase ozonated PPMM grafted with PEG of different molecular weight 
Additional peaks 1, 2, 3, 4 appeared after the AAm grafted

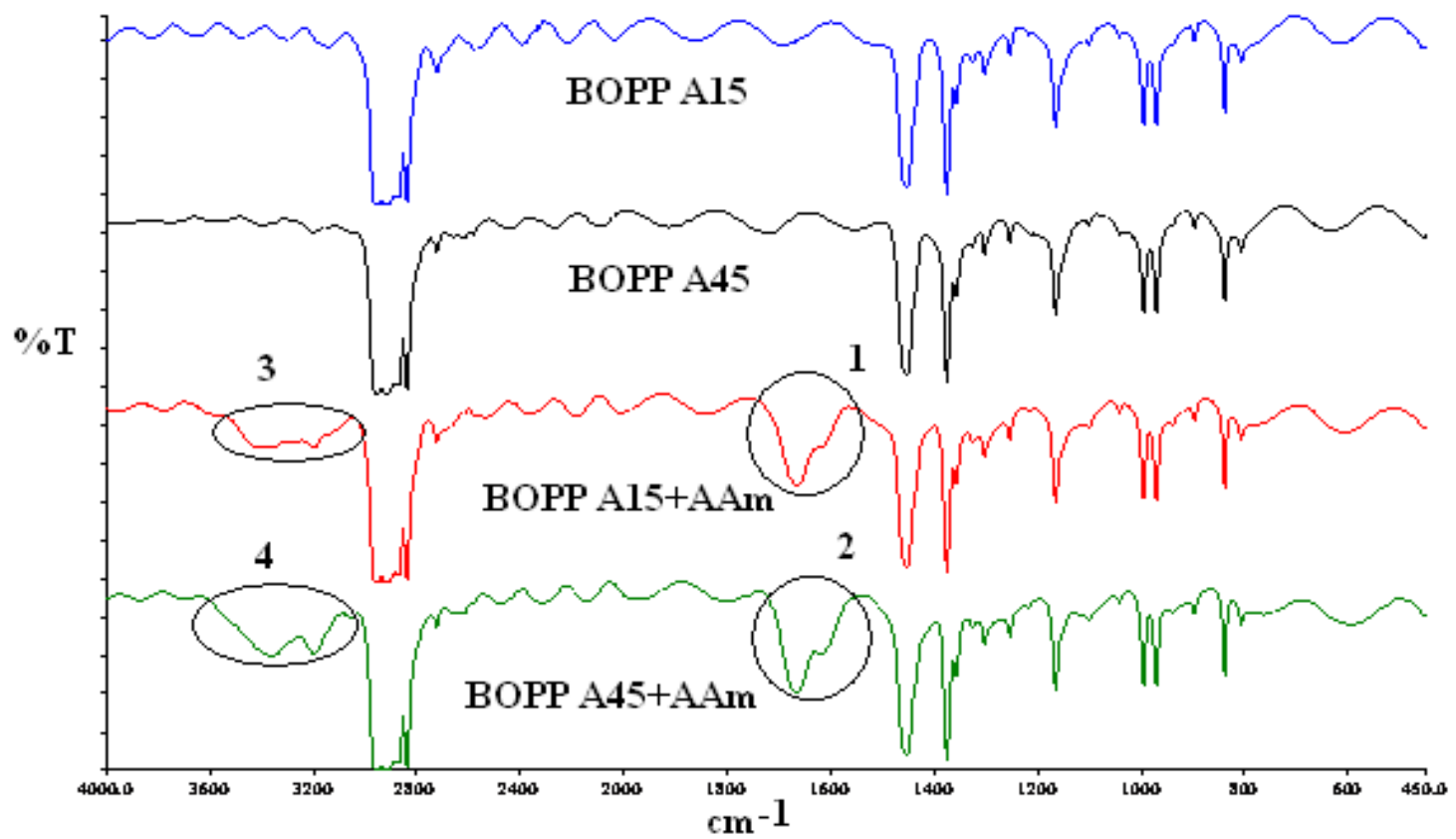

Fig 4.37 FTIR of BOPP with 3.0 wt\% applied ozone dose for A15, A45 + AAm graft polymerization (A15 and A45 $=15$ - and 45-minute ozonation times in the aqueous phase, respectively.)

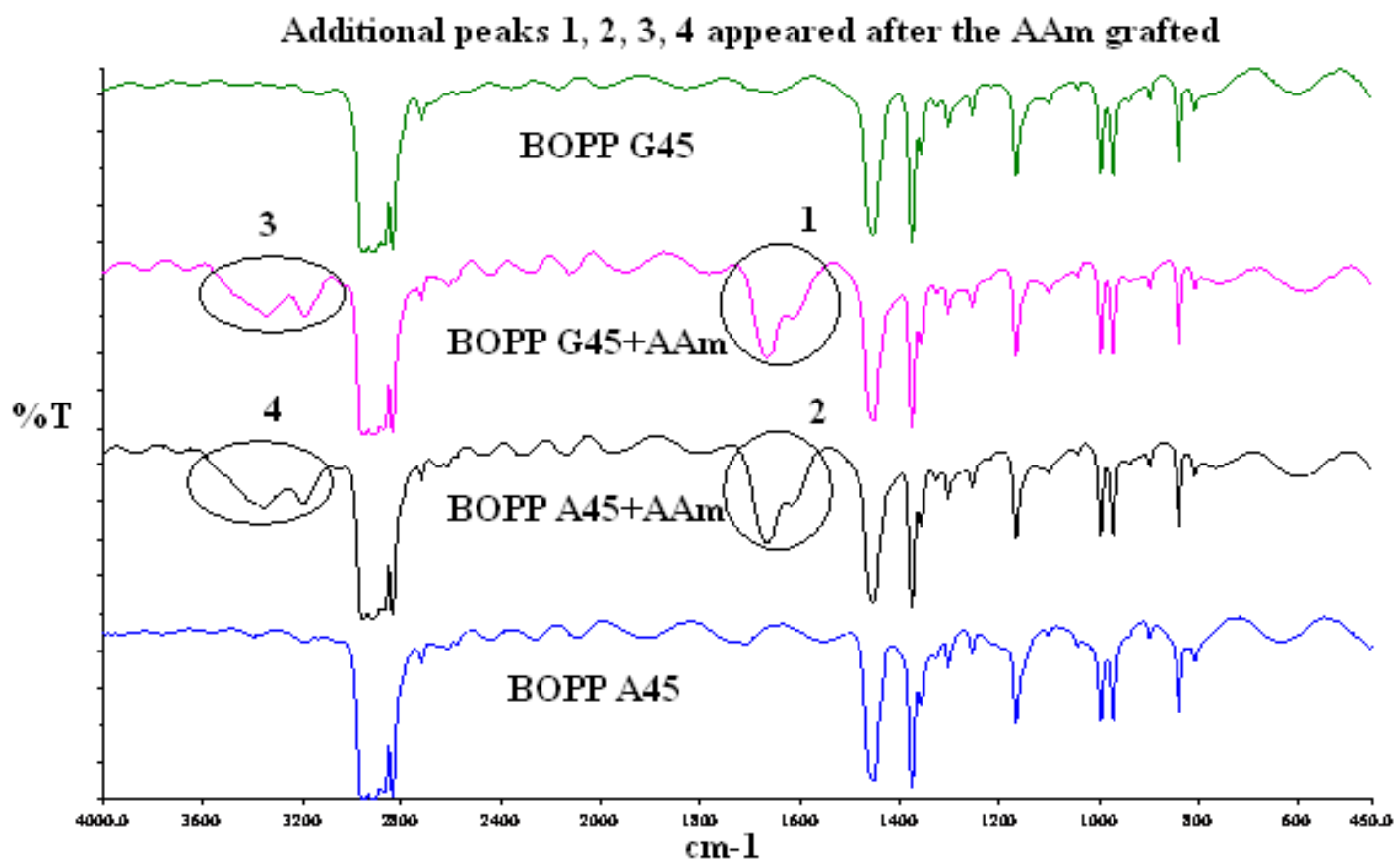

Fig 4.38 FTIR of BOPP with $3.0 \mathrm{wt} \%$ applied ozone dose for A45 or G45, plus AAm graft polymerization $(\mathrm{G} 45=45$ min ozonation time in the gaseous phase. 


\subsubsection{AAm grafedt to BOPP and FTIR analysis}

The same reaction conditions as Section 4.3.1.1 were used, and ozonated PPMM was replaced by BOPP. The washed and dried samples were sent for FTIR analysis. The functional groups on the film surfaces were then characterized. The FTIR results of polypropylene films ozonated by different ozonation time were reported by $\mathrm{Gu}$ (2008) in the master thesis. Figure 4.37 shows the FTIR spectra of the ozonated and AAm grafted BOPP in the aqueous phase with different ozonation times; Figure 4.38 shows the FTIR spectra comparison of the films for which AAm graft polymerization was induced by aqueous and gaseous phases' ozonation. A strong peak appeared at $1706 \mathrm{~cm}^{-1}(\mathrm{C}=\mathrm{O}$ group $)$ and a weak, wide peak appeared at 3200-3400 $\mathrm{cm}^{-1}$ (O-H group) after grafting with AAm. From Figure 4.37 , the characteristic peaks, which became larger and deeper as ozonation time increased, showed that the grafting of the monomer AAm to the film was greater when there was a longer ozonation time, which resulted in the existence of more active points on the film for the AAm to graft to.

\subsubsection{Graft degree}

Graft degree is the weight change ratio after the graft polymerization, which is a percentage of the increased weight after graft polymerization (Equation 2.41). Therefore, the graft degree indicates the amount of monomer/oligomer grafting.

Table 4.3 Graft degree of the PPMM with monomers AAm and PEG

\begin{tabular}{l|lcccc}
\hline $\begin{array}{c}\text { Aqueous } \\
\text { ozonation }\end{array}$ & Membrane & $\begin{array}{c}\text { Average } \\
\text { weight before } \\
\text { grafting (g) }\end{array}$ & $\begin{array}{c}\text { Monomers } \\
\text { grafted }\end{array}$ & $\begin{array}{c}\text { Average } \\
\text { weight after } \\
\text { grafting (g) }\end{array}$ & $\begin{array}{l}\text { Graft } \\
\text { degree }\end{array}$ \\
\hline $3.00 \mathrm{wt} \%$ & PPMM & 0.0440 & AAm & 0.0456 & $3.75 \%$ \\
$15 \mathrm{~min}$ & PPMM & 0.0450 & PEG-200 & 0.0462 & $2.55 \%$ \\
& PPMM & 0.0455 & PEG-600 & 0.0470 & $3.19 \%$ \\
& PPMM & 0.0453 & PEG-3350 & 0.0468 & $3.31 \%$ \\
\hline
\end{tabular}

\subsubsection{Graft degree of PPMM}

The weights of the virgin and grafted membranes were determined, and the graft degree was calculated as shown in Table 4.3. The results show that the average graft degree 
(two replicas for each test) was from $2.55 \%$ to $3.75 \%$ of these monomers' grafting induced by the ozonation ( $3.0 \mathrm{wt} \%$ ozone and 15- minute ozonation time). The AAm had slightly higher graft degree than the PEG had.

\subsubsection{Graft degree of BOPP}

The weights of the virgin and graft BOPP films were checked, and the graft degree were calculated as shown in Table 4.4. The results showed that the average graft degree (two sample replicas did for each test) was from $2.33 \%$ to $3.26 \%$ of the monomer AAm graft induced by the ozonation (3.00 wt\% ozone and 18- and 45-minute ozonation times). This demonstrated a correlation between the graft degree and the ozonation time.

Table 4.4 Graft degree of BOPP with the monomer AAm

\begin{tabular}{c|cccccc}
\hline $\begin{array}{c}\text { Aqueous } \\
\mathrm{O}_{3}\end{array}$ & Film & $\begin{array}{c}\text { Ozonation } \\
\text { time } \\
(\mathrm{min})\end{array}$ & $\begin{array}{c}\text { Average } \\
\text { weight before } \\
\text { grafting (g) }\end{array}$ & $\begin{array}{c}\text { Monomers } \\
\text { grafted }\end{array}$ & $\begin{array}{c}\text { Average } \\
\text { weight after } \\
\text { grafting (g) }\end{array}$ & $\begin{array}{c}\text { Graft } \\
\text { degree }\end{array}$ \\
\hline $3.0 \mathrm{wt} \%$ & BOPP & 18 & 0.0860 & AAm & 0.0880 & $2.33 \%$ \\
& BOPP & 45 & 0.0920 & AAm & 0.0950 & $3.26 \%$ \\
\hline
\end{tabular}

\subsubsection{Contact angle test}

Contact angle measurement is the method to measure the surface tension. When the contact angle is low, the hydrophilicity of substrate is high.

\subsubsection{Contact angle of the AAm grafted PPMM}

As shown in Table 4.5, both the aqueous and gaseous phases' ozonation decreased the contact angle of PPMM, AAm graft polymerization enhanced this trend, the maximum decrease of the contact angle reduced from $126.9^{\circ}$ to $92.2^{\circ}$ by 20 minutes aqueous phase's ozonation plus AAm graft polymerization. The tested result of contact angle by the gaseous phase's ozonation was larger than that by the aqueous phase ozonation at the same ozone dose, and the contact angle of AAm grafted PPMM by the aqueous phase's ozonation was smaller than that by the gaseous phase's ozonation. The data indicated that hydrophilicity improved more by the aqueous ozonation and AAm graft polymerization, the aqueous phase 
ozonation was more effective.

Table 4.5 Contact angle of the PPMM modified by ozonation and AAm graft

\begin{tabular}{l|cccc}
\hline $\begin{array}{l}\text { Contact angle } \\
\text { virgin PPMM }\end{array}$ & 128.8 & & & \\
\hline Contact angle & A6 & A15 & G6 & G15 \\
before grafting & 122.7 & 120.6 & 114.9 & 106.6 \\
\hline Contact angle & A6+AAm & A15+AAm & G6+AAm & G15+AAm \\
after grafting & 92.1 & 92.5 & 108.4 & 99.1 \\
\hline
\end{tabular}

Remarks:

A6 or A15: $\quad$ Aqueous phase ozonation in 6 minutes, or 15 minutes

G6 or G15: $\quad$ Gaseous phase ozonation in 6 minutes, or 15 minutes

A6 or A15+AAm: Aqueous phase ozonation and AAm graft polymerization

G6 or G15+AAm: Gaseous phase ozonation and AAm graft polymerization

\subsubsection{Contact angle of the HEMA grafted PPMM}

The test results of contact angle of HEMA grafted PPMM are shown in Table 4.6, the resulted contact angle by the gaseous phase's ozonation and HEMA graft was higher than that by the aqueous phase's ozonation at the same dose. The data indicated that hydrophilicity improved more by the aqueous ozonation and HEMA graft polymerization, the aqueous phase's ozonation is more effective.

Table 4.6 Contact angle of the PPMM modified by ozonation and HEMA graft

\begin{tabular}{c|cc}
\hline $\begin{array}{c}\text { Virgin PPMM } \\
\text { average }\end{array}$ & $\begin{array}{c}\text { A18+HEMA } \\
\text { average }\end{array}$ & $\begin{array}{c}\text { G18+HEMA } \\
\text { average }\end{array}$ \\
\hline 128.2 & 90.1 & 107.8 \\
\hline
\end{tabular}

Remarks:

A18+HEMA: Aqueous phase ozonation in 18 minutes, and HEMA graft polymerization

G18+HEMA Gaseous phase ozonation in 18 minutes, and HEMA graft polymerization

\subsubsection{Contact angle of the PEG grafted PPMM}

The test results of contact angle of PEG grafted PPMM are shown in Table 4.7, which showed PEG200 grafted membrane had the highest hydrophilicity. This phenomenon also indicated the monomer PEG 200 has the best accessibility to the active sites. 
Table 4.7 Contact angle of the PEG graft on PPMM

\begin{tabular}{c|ccc}
\hline $\begin{array}{c}\text { Average } \\
\text { Virgin PPMM }\end{array}$ & $\begin{array}{c}\text { Average } \\
\text { A18+PEG3350 }\end{array}$ & $\begin{array}{c}\text { Average } \\
\text { A18+PEG600 }\end{array}$ & $\begin{array}{c}\text { Average } \\
\text { A18+PEG200 }\end{array}$ \\
\hline 128.9 & 107.4 & 104.5 & 102.1 \\
\hline
\end{tabular}

Remarks:

A18+PEG3350: Aqueous phase ozonation in 18 minutes, and PEG3350graft polymerization A18+PEG600 Aqueous phase ozonation in 18 minutes, and PEG600 graft polymerization

A18+PEG200 Aqueous phase ozonation in 18 minutes, and PEG200 graft polymerization

\subsubsection{Contact angle of the AAm grafted BOPP}

The test results of contact angle by the aqueous phase's ozonation with different time are shown in Table 4.8, contact angle decreased after the aqueous phase's ozonation; decreased more after AAm grafted. The contact angle went down the most after AAm grafted to the 45 minutes aqueous phase's ozonated BOPP film. The data indicated that hydrophilicity improved significantly $(-35.7 \%)$ by the aqueous phase's ozonation and AAm graft polymerization.

Table 4.8 Contact angle of the BOPP film modified by ozonation and AAm graft

\begin{tabular}{c|cccc}
\hline BOPP film $20 \mu \mathrm{m}$ & \multicolumn{4}{|c}{ Average Contact angle } \\
\hline Virgin BOPP & A18 & A45 & A18+AAm & A45+AAm \\
\hline 87.4 & 79.2 & 79.0 & 66.6 & 56.2 \\
\hline Remarks: & & & \\
A18 or A45: & Aqueous phase ozonation in 18 minutes, or 45 minutes \\
A18 or A45+AAm: & Aqueous phase ozonation and AAm graft polymerization
\end{tabular}

\subsubsection{X-ray diffraction (XRD) analysis}

English physicists Sir W.H. Bragg and his son Sir W.L. Bragg developed a relationship in 1913 to explain why the cleavage faces of crystals appear to reflect X-ray beams at certain angles of incidence (theta, $\theta$ ). Bragg's Law shows that $n \lambda=2 d \sin \theta$. The variable $d$ is the distance between atomic layers in a crystal, and the variable lambda $\lambda$ is the wavelength of the incident $\mathrm{X}$-ray beam; $\mathrm{n}$ is an integer (a whole number of wavelengths $\lambda$ for the pathway). This observation is an example of X-ray wave interference, commonly known as X-ray diffraction (Nuffield, 1966).

The broad feature in the $14-30^{\circ} 2 \theta$ region remains for all the samples, XRD peaks of 
polypropylene appear at 5 values in $2 \theta$ region $14.25-28.75^{\circ}$. Results of diffractogram peaks' counts, as shown in Figure 4.39, indicated that ozonation decreased the surface crystallinity of PPMM and increased the amorphicity, and crystalline decreased with ozonation time (the higher counts represented the high crystallinity). This phenomenon is very similar to the results of Michael et al. (2004) for the polyester fabrics.

Results of diffractogram peaks' counts, as shown in Figure 4.39, Figure 4.40, and Figure 4.41 indicate that ozonation decreased the crystalline, while AAm graft increased the crystalline. AAm grafted membranes by the aqueous phase ozonation had the largest crystalline increase (Graft degree of A15+AAm was 3.75\%, the peak count was about 3600 correlated to the large XRD peak of grafted PPMM). Unlike small molecules, such as metal or methane, which is either totally crystalline or totally amorphous, polymer molecules are often only partially crystalline due to their sizes, having crystalline regions dispersed within the amorphous material. The small crystalline regions have a precise alignment. These crystals can be regularly shaped as thin platelets, and form multilayer structure, or form spherulites. Any chain disorder or misalignment will result in an amorphous region (Callister, 2006). The ozonation inserted oxygen atoms into chain molecules or broke the chains on the surfaces, disordered the chain, thus decreased the crystalline. AAm itself can be totally crystallized. When grafted onto the surfaces, AAm made the molecule chains of polymer easy to crystallize, therefore increased the crystalline. The brought in different atoms by ozonation or graft polymerization changed some molecules' status, thus shifted the peak a little. 
The relationship of X-ray intensity and 20 degrees of a Quartz sample using X-Ray Diffractometry-PP Virgin- A6-A15

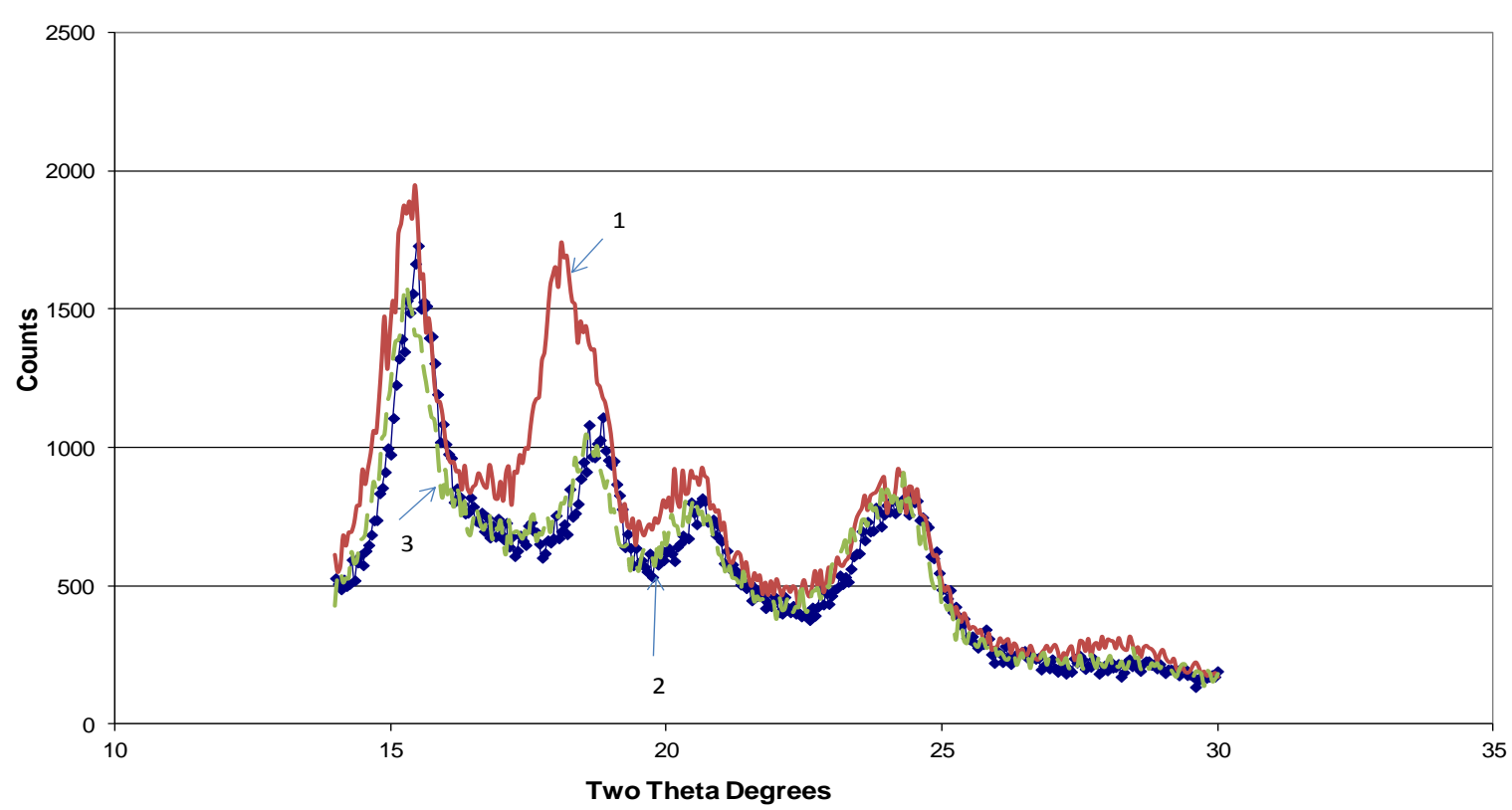

Fig 4.39 XRD of PPMM: 1 - virgin; 2 - 6 min aqueous ozonation; 3-15 min aqueous ozonation

The relationship of X-ray intensity and 20 degrees of a Quartz sample using X-Ray Diffractometry - PP virgin+A15+AAM

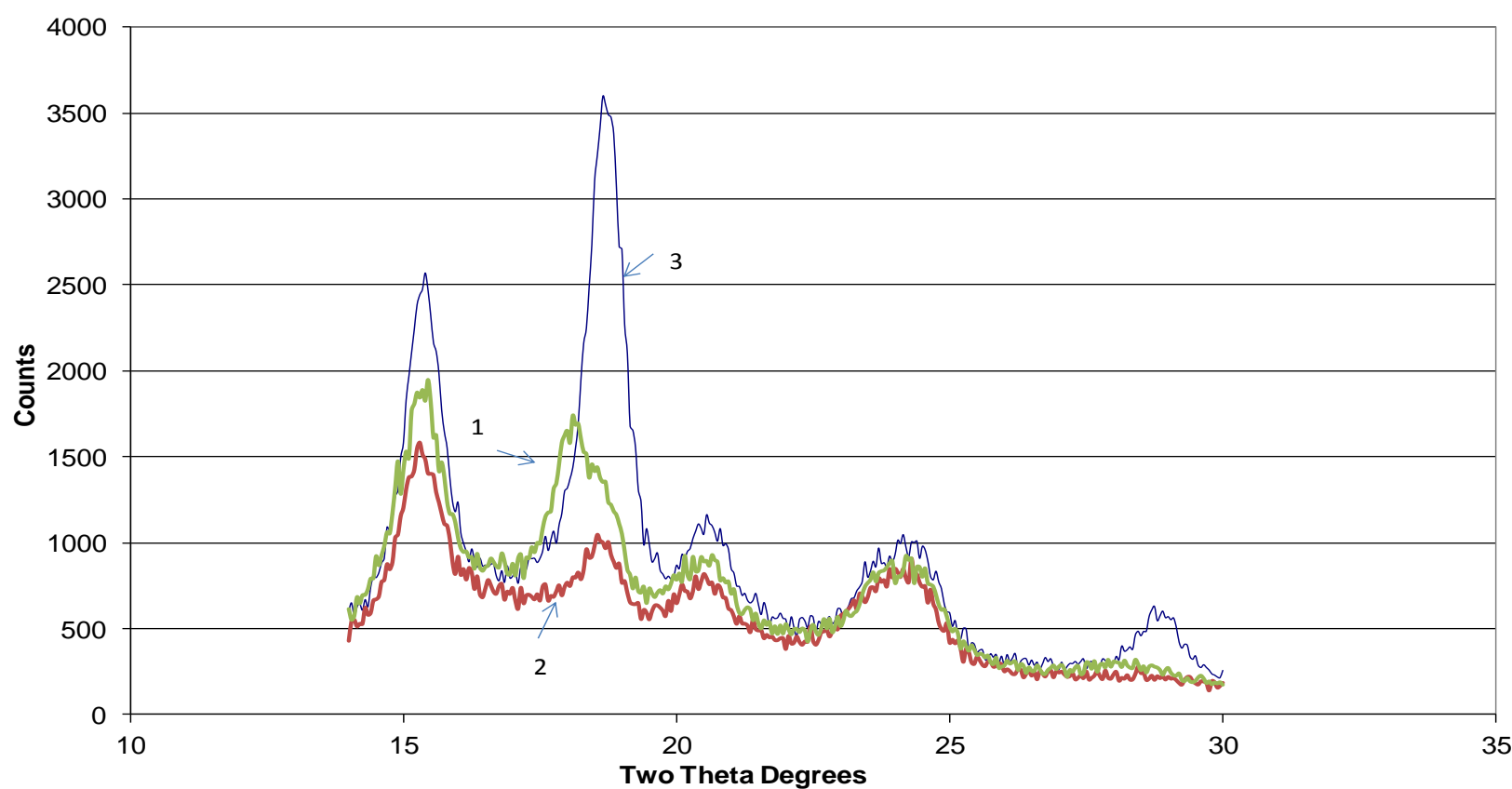

Fig 4.40 XRD of PPMM: 1 - virgin; 2 - 15 min aqueous ozonation; 3-15 min aqueous ozonation plus AAm graft 


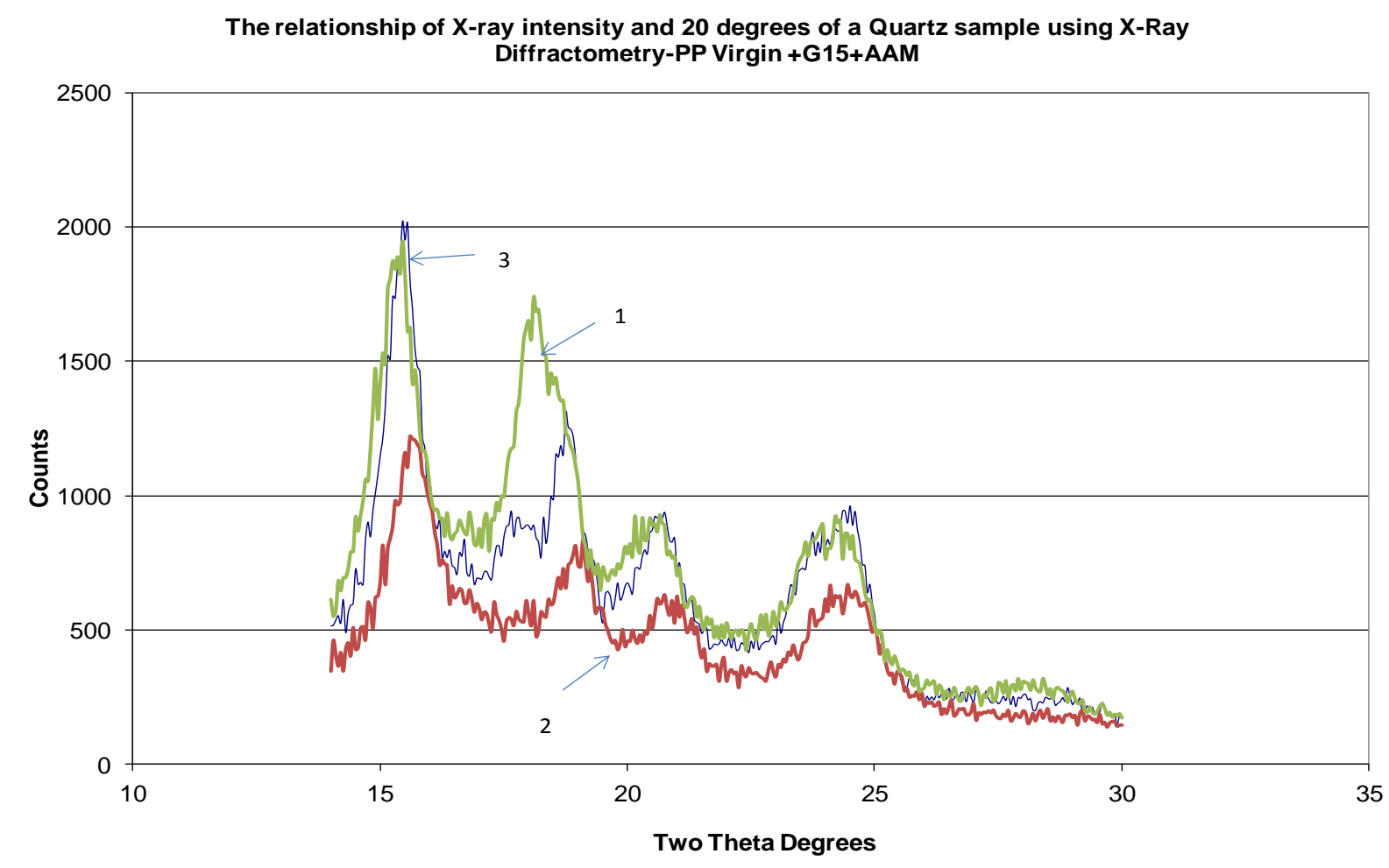

Fig 4.41 XRD of PPMM: 1 - virgin; 2 - 15 min gaseous ozonation; 3 - 15 min gaseous ozonation plus AAm graft

\subsection{Protein Adsorption and Filtration Flux Test}

Bovine serum albumin (BSA, Sigma, USA) was used as a model protein to evaluate the protein fouling characteristics of virgin and grafted membranes. The membranes were soaked in IPA for $1 \mathrm{~min}$ for pre-wetting and washed with $500 \mathrm{ml}$ of distilled water for 5 minutes, then fixed to the flux test device, as shown in Figure 3.5. A BSA solution with a concentration of $1000 \mathrm{mg} / \mathrm{L}$, and $\mathrm{pH}$ adjusted to 6.9 with a $0.1 \mathrm{M}$ phosphate buffer, was used for the filtration test (Hu et al., 2008; Wang et al., 1999). The initial pumping pressure of the solution was set at $10 \mathrm{psig}$ and adjusted to maintain this pressure, and the filtration of 900-1000 ml of solution was run till the permeate flow declined to a very low number. The DW back flush (back flush applied to move the cake layer and absorption fouling) pressure was set at 20 psig and maintained at this pressure, and the amount of DW used was $600 \mathrm{ml}$. The solution permeate weights of the membranes were recorded every 5 seconds. The recorded weights of the modified membranes were calculated along with flow rate and plotted against time, then compared to that of the virgin membrane. The results were 
presented in the following paragraphs.

\subsubsection{AAm grafted PPMM}

As described in Section 3.4.4.1, the dead-end filtration test was applied to the AAm grafted PPMM, and the permeate flow rate versus time (shown in Figure 4.42) was compared to that of virgin PPMM. It was found that the aqueous phase ozonation and AAm grafting resulted in the PPMM having a higher permeate flow rate, and the permeate flow rate of this modified membrane kept higher comparing to the gaseous counterpart and virgin PPMM during the BSA filtration test. A18 = 18 minutes of aqueous phase ozonation; G18 = 18 minutes of gaseous phase ozonation; AAm = AAm graft polymerization.

The back flush was implemented by reversing the fouled membranes and using DW to flush the membrane with $600 \mathrm{ml}$ of permeate and double the pressure (20 psig) of the BSA filtration.

The cleaned membranes were then used in the second filtration test using BSA solution. The chart of permeate versus time is shown in Figure 4.43. The aqueous phase ozonated and AAm grafted membrane was found to have the best recovery of permeate flow rate, a $84 \%(1.47 \mathrm{ml} / \mathrm{s} \div 1.75 \mathrm{ml} / \mathrm{s})$ permeate flow rate was recovered after the back flush; while the gaseous phase ozonated and AAm grafted membrane was found to have the permeate flow rate recovery of $82 \%(1.27 \mathrm{ml} / \mathrm{s} \div 1.54 \mathrm{ml} / \mathrm{s})$; the virgin membrane was found to have the least permeate flow rate recovery of $52 \%(0.58 \mathrm{ml} / \mathrm{s} \div 1.1 \mathrm{ml} / \mathrm{s})$. These results indicated that the aqueous phase ozonated and AAm grafted membrane had the least absorption fouling-the improved hydrophilicity repelled the BSA absorption, and it was found easier for this modified membrane to clean by back flush, thus it had a longer life time and a better anti-fouling property.

The explanation for the phenomenon, which the membrane with higher permeate flow rate had a faster flow rate dropping, is that the cake layer piled up faster when more solution was filtered by the membrane. 


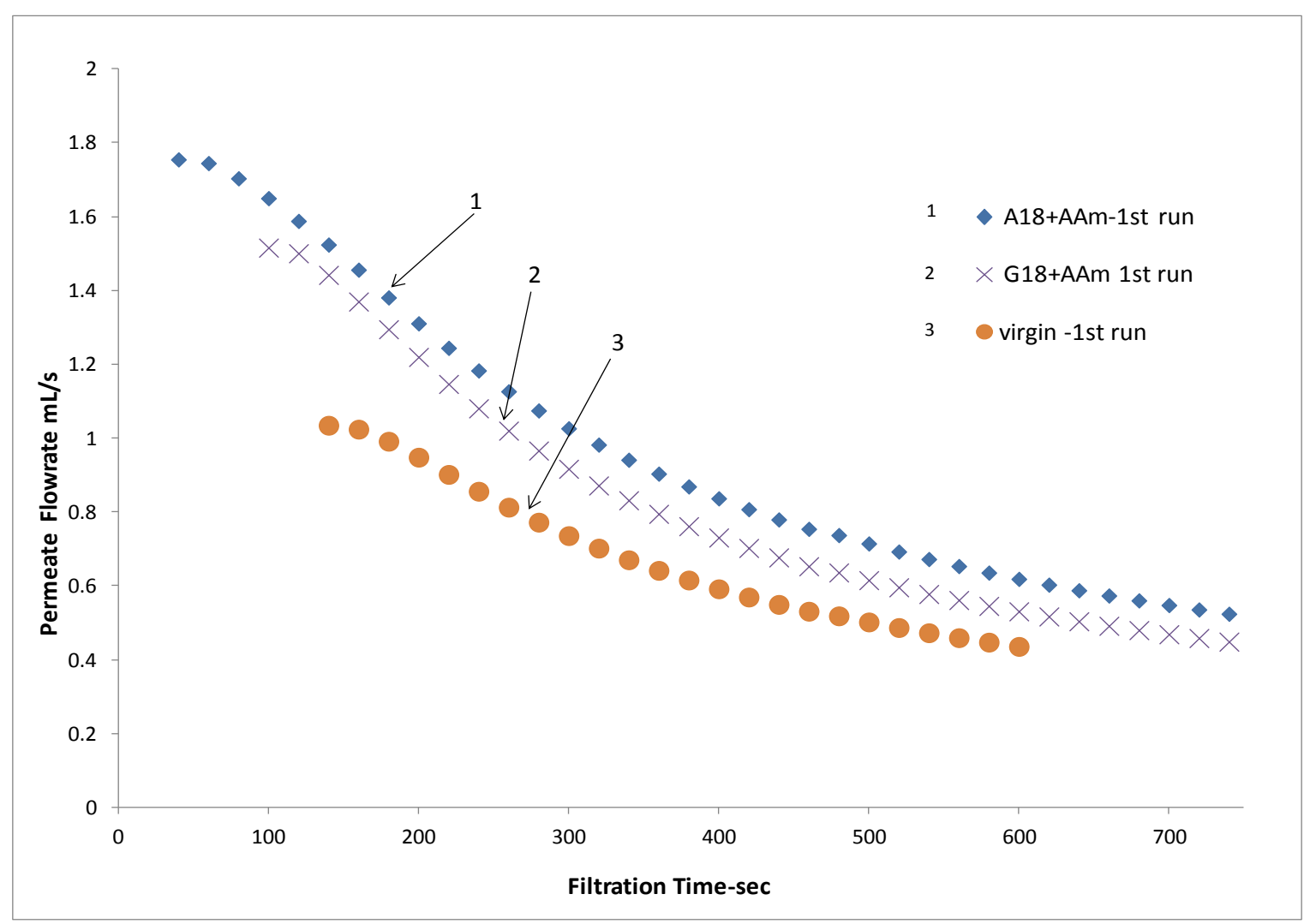

Fig 4.42 Dead-end BSA filtarion of virgin, A18, and G18 + AAm modified PPMM

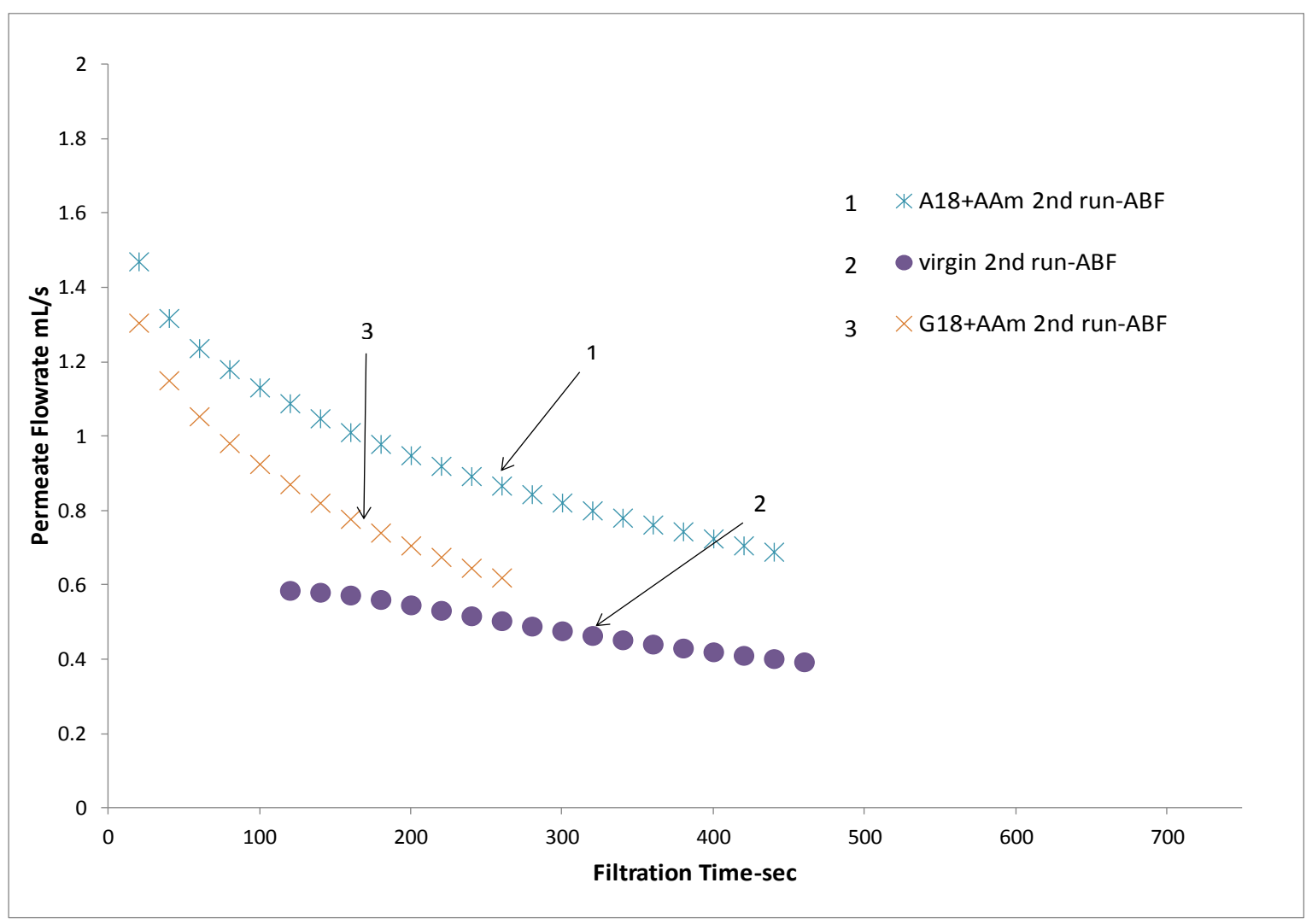

Fig 4.43 Second filtration test (after back flush-ABF) of AAm modified PPMM 


\subsubsection{PEG grafted PPMM}

Different PEG grafted PPMM were used to test filtration through the dead-end filtration device, as shown in Figure 4.44. The PEG200 and PEG600 modified membranes had the best permeate flow rates, and the PEG3350 modified PPMM also had a better permeate flow rate than the virgin PPMM, which indicate that the PEG grafting improved the anti-fouling property of PPMM. It was found from Figure 4.44 that the PEG200 grafted PPMM had the biggest permeate flow rate -around $3.0 \mathrm{ml} / \mathrm{s}$, while the PEG3350 modified PPMM had the least improvement, which indicated that the PEG200 modified PPMM had the best anti-fouling property to BSA solution. The reason is that the PEG200 modified PPMM had a lower contact angle, as shown in Table 4.7 at Section 4.3.3.3, thus its hydrophilicity was improved more. Another reason could be that PEG 3350 has a larger molecular size than PEG200 and PEG600, and was easier to block the pores, or reduced the pore size, when it grafted onto the PPMM surface or inside the pores. The flow rate of virgin PPMM was a little different from that showed in Section 4.4.1, because the BSA is a protein, the molecule size and filtration condition can vary a little. The comparison of filtration in the same section was conducted using the same BSA solution, while BSA solution was freshly made in different sections.

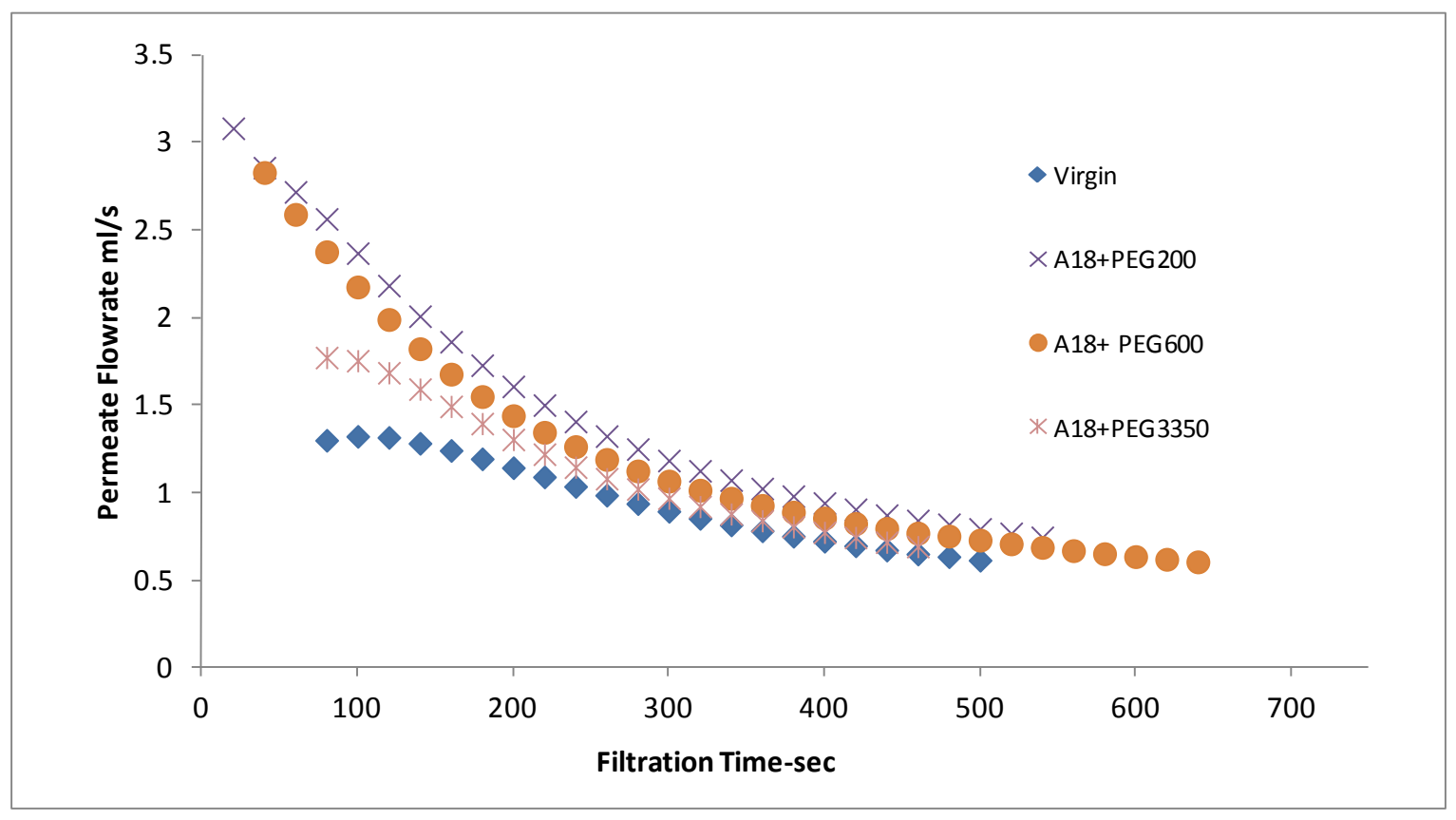

Fig 4.44 BSA filtration test of PEG modified PPMM 


\subsubsection{HEMA grafted PPMM}

HEMA grafted PPMM were used to test filtration through the dead-end filtration device, as shown in Figure 4.45. HEMA grafted membranes had a higher permeate flow rate than virgin PPMM, which indicate the HEMA grafting improved the anti-fouling property of PPMM.

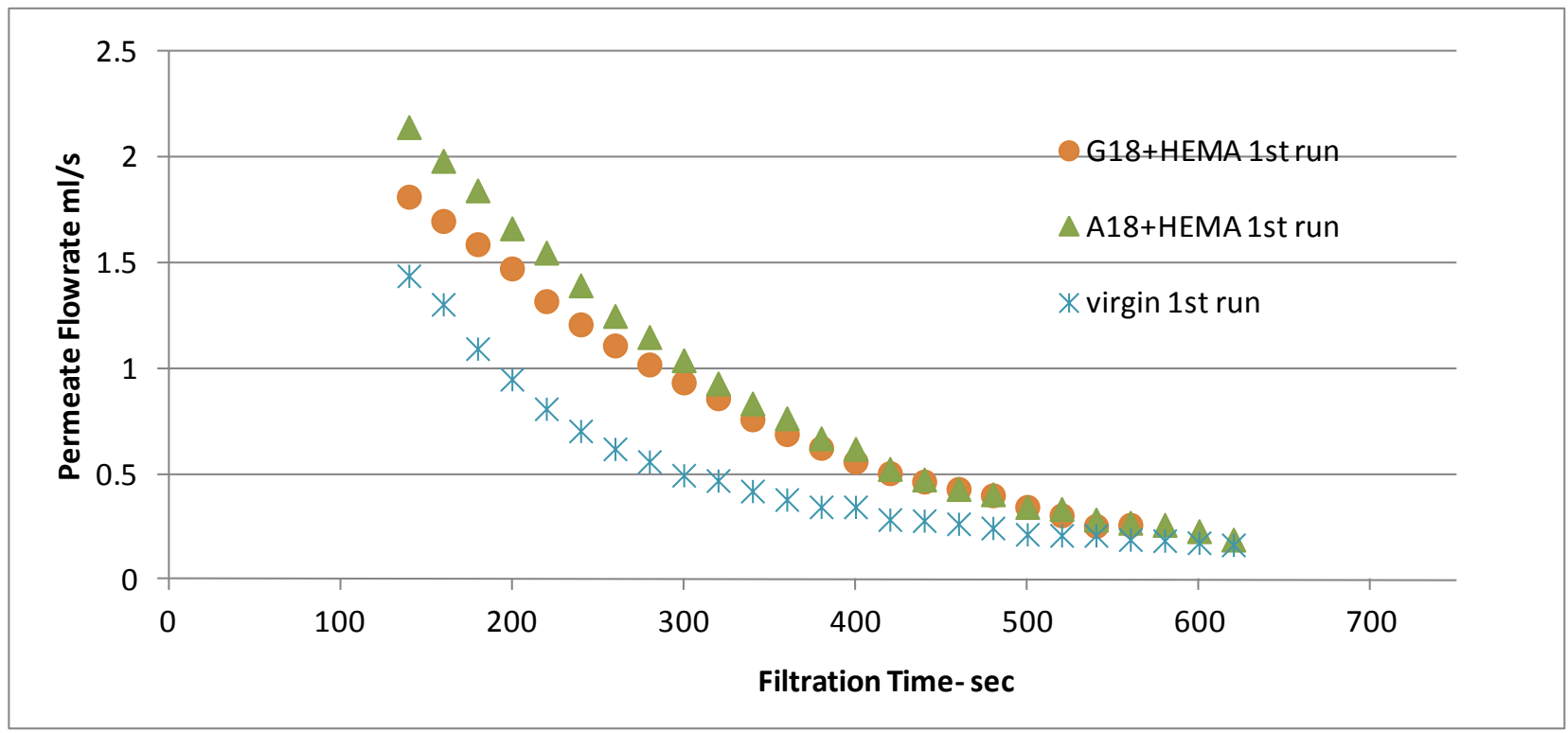

Fig 4.45 BSA filtration test of HEMA modified PPMM

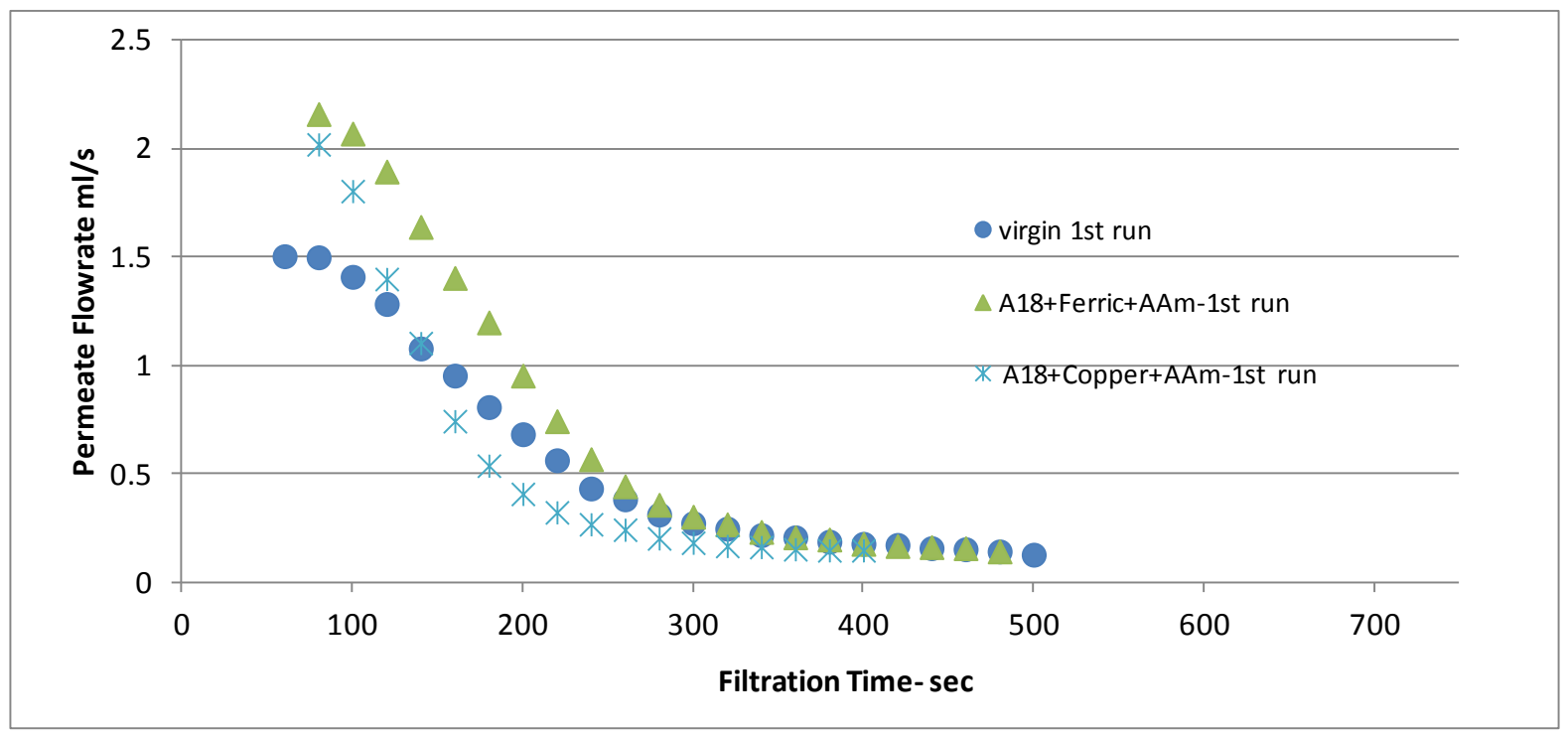

Fig 4.46 BSA filtration test of $\mathrm{Fe}^{3+}$ and $\mathrm{Cu}^{2+}+\mathrm{AAm}$ modified PPMM 


\subsubsection{Catalytic grafted PPMM}

PPMM with AAm geafted after ozonation either in the presence of $0.05 \mathrm{~g} / \mathrm{L}(0.7868$ $\mathrm{mmol} / \mathrm{L}) \mathrm{Cu}^{2+}$ catalyst or $0.1 \mathrm{~g} / \mathrm{L}(1.574 \mathrm{mmol} / \mathrm{L}) \mathrm{Fe}^{3+}$ catalyst was used for filtration through the dead-end filtration device, and compared with virgin PPMM. As shown in Figure 4.46, PPMM with AAm grafted had a higher permeate flow rate than virgin PPMM.

It is confirmed experimentally in this study that modification of PPMM by ozonation and grafting monomers, such as AAm, PEG, and HEMA, did improve the hydrophilicity of PPMM surfaces, thus the anti-fouling property was improved. Furthermore, the PEG200 grafted PPMM showed the largest permeate flow rate, which indicated that it had the best hydrophilicity improvement. Catalyzed ozonation helped the AAm grafting polymerization, and got a larger permeate flow rate of BSA solution than the non-catalyzed counterpart.

\subsubsection{Formula of filtration flux and fouling}

In filtration tests, the flux formula for pore blockage and cake layer combination fouling, refer to Equation 2.53, in which the relationship of flow rate (g/s) and filtration time (s) is represented by the following equation (Kilduff et al., 2005):

$Q=Q_{o}\left[\exp \left(-\frac{\alpha_{b} \Delta P C_{f}}{R_{m}} t\right)+\frac{R_{m}}{R_{m}+R_{c}} \times\left(1-\exp \left(-\frac{\alpha_{b} \Delta P C_{f}}{R_{m}} t\right)\right)\right]$

where $\mathrm{Q}$ is the permeate flow rate, and other parameters were defined in Section 2.6.3

Therefore,

$Q=Q_{o}\left[\frac{R_{c}}{R_{m}+R_{c}} \exp \left(-\frac{\alpha_{b} \Delta P C_{f}}{R_{m}} t\right)+\frac{R_{m}}{R_{m}+R_{c}}\right]$

Refers to equation (2.54), 


$$
\begin{aligned}
& \frac{R_{m}+R_{c}}{R_{m}+R_{c, 0}}=\sqrt{1+\frac{2 \alpha_{c} \Delta P C_{f}}{\left(R_{m}+R_{c . o}\right)^{2}}} t \\
& R_{c}=\left(R_{m}+R_{c, o}\right) \sqrt{1+\frac{2 \alpha_{c} \Delta P C_{f}}{\left(R_{m}+R_{c . o}\right)^{2}}} t-R_{m}
\end{aligned}
$$

Combination of Equation (4.3) and (4.4) yields,

$$
Q=Q_{o}\left[\frac{R_{c}}{R_{m}+R_{c}} \exp \left(-\frac{\alpha_{b} \Delta P C_{f}}{R_{m}} t\right)+\frac{R_{m}}{R_{m}+R_{c}}\right]
$$

$$
\begin{aligned}
& =Q_{o} R_{m} /\left[\left(R_{m}+R_{c, o}\right) \sqrt{1+\frac{2 \alpha_{c} \Delta P C_{f}}{\left(R_{m}+R_{c . o}\right)^{2}}} t\left[1-\exp \left(-\frac{\alpha_{b} \Delta P C_{f}}{R_{m}} t\right)\right]+Q_{o} \exp \left(-\frac{\alpha_{b} \Delta P C_{f}}{R_{m}} t\right)\right. \\
& =Q_{o}\left[k_{1} \sqrt{1+k_{2} t}+\left(1-k_{1} \sqrt{1+k_{2} t}\right) \exp \left(-k_{3} t\right)\right]
\end{aligned}
$$

where $k_{l=} R_{m} /\left[\left(R_{m}+R_{c, o}\right) ; k_{2}=\frac{2 \alpha_{c} \Delta P C_{f}}{\left(R_{m}+R_{c, o}\right)^{2}} ; k_{3}=\frac{\alpha_{b} \Delta P C_{f}}{R_{m}}\right.$

The permeate flow rate with time is a complex exponential relationship. The $k_{1}, k_{2}, k_{3}$ can be calculated out from Figures 4.4.2 to 4.4.6 by using a certain computer program, this project will be left for further study.

\subsection{SEM Images}

The scanning electron microscope (SEM) is an instrument used to observe surface morphology and get images of a larger magnification by electron scanning of the surface.

\subsubsection{SEM images of AAm grafted PPMM}

The surface morphology of the PPMM before and after AAm graft polymerization 
was observed using SEM. Figure 4.47 shows the SEM images of virgin, ozonated, and AAm-grafted PP membranes at 5000x magnification. It can be observed that ozonation changed the morphology of the membranes. In Figures $4.47 \mathrm{~d}$ and $4.47 \mathrm{e}$, one can see AAm grafted on the surface and in the pores of the PPMM, which modified its hydrophilicity. The AAm-grafted PPMM by aqueous phase ozonation had clearer pores and less blocking in the images than that grafted by gaseous phase ozonation.

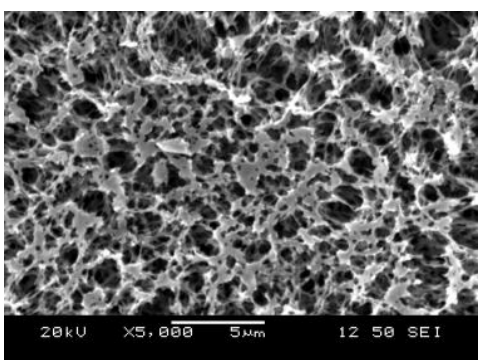

a.

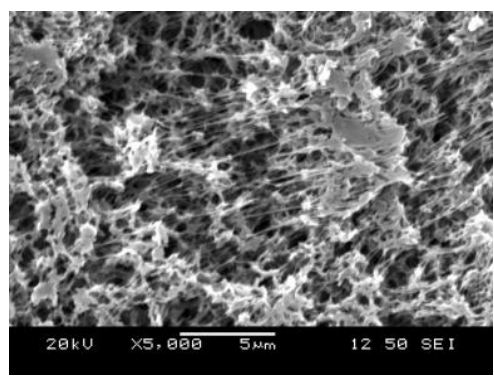

d.

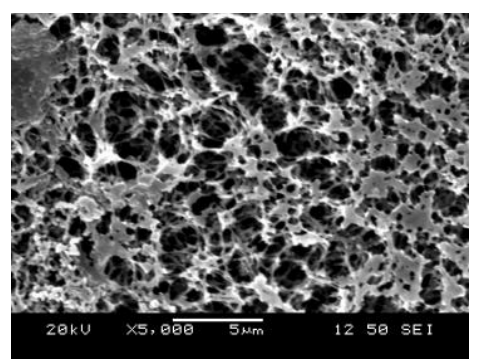

b.

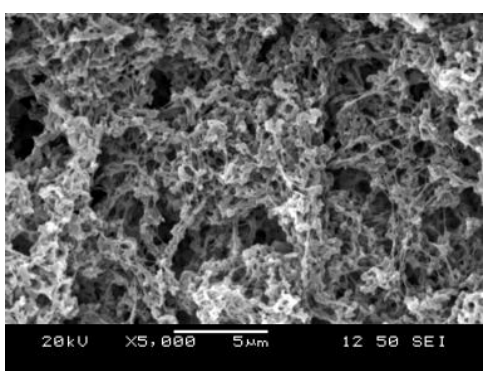

e.

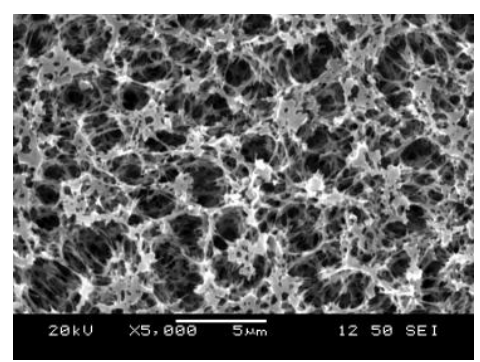

c.

Fig 4.47 SEM images of PPMM grafted by AAm, a. Virgin PPMM, b. Ozonated in aqueous solution for $15 \mathrm{~min}$ (A15), c. Ozonated in gaseous solution for $15 \mathrm{~min}$ (G15), d. After A15 and AAm graft, and e. After G15 and AAm graft

\subsubsection{SEM images of the BSA filtration test for PPMM grafted by AAm}

The surface morphology of PPMM before and after AAm graft polymerization and the protein (BSA) filtration test was observed using SEM. Figure 4.48 shows the SEM images of virgin, gaseous and aqueous phase ozonated, and AAm-grafted PP membranes at 300x magnification. As shown in Figures 4.48a, 4.48b, and 4.48c, BSA fouled the surfaces of virgin, G18 + AAm, and A18 + AAm PP microfiltration membranes. Figures 4.48d, 4.48e, and 4.48f show washed* BSA fouled virgin, G18 + AAm, and A18 + AAm PP microfiltration 
membranes. The cake layer fouling was removed and the pores can be seen clearly after the fouled AAm grafted membranes have been washed (Figures 4.38e and 4.38f), while the pores of the unmodified membranes were barely visible after the same treatment (Figure 4.48d). These images indicate that AAm grafting improved the anti-fouling property of the membranes, and the aqueous phase ozonation followed by AAm grafting improved it the most.

*Wash method: the membranes were soaked in DW and the surfaces were abraded 3 times using a clean, wetted napkin.

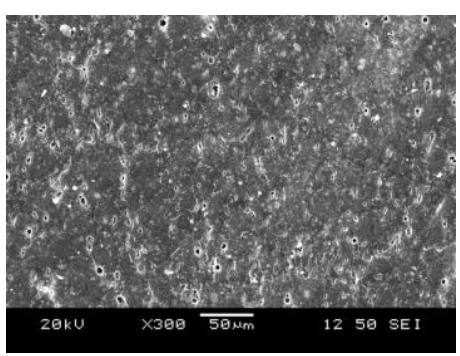

a.

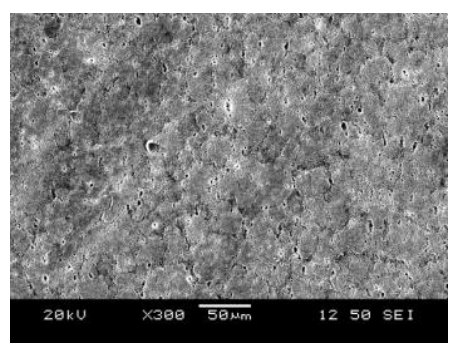

d.

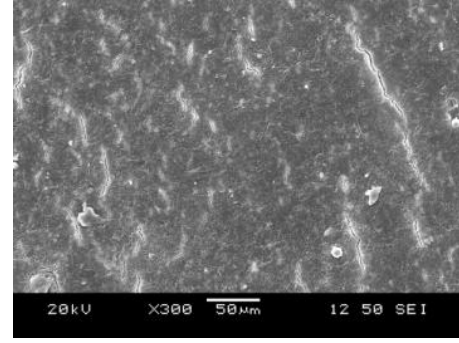

b.

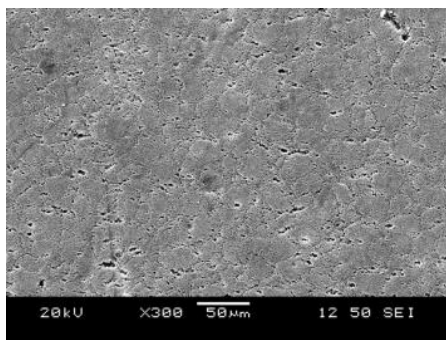

e.

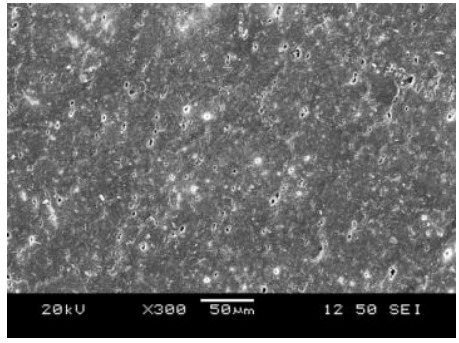

c.

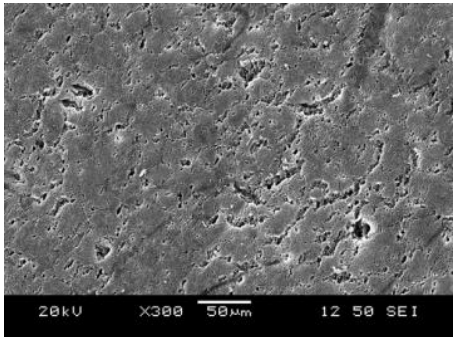

f.

Fig 4.48 SEM images of BSA fouled PPMM, a. Virgin PPMM, BSA fouled - non washed, b. G18 + AAm, BSA fouled - non washed, c. A18 + AAm, BSA fouled - non washed d. Virgin PPMM, BSA fouled - washed, e. G18 + AAm, BSA fouled - washed, and f. A18 + AAm, BSA fouled - washed

\subsubsection{SEM images of BOPP after AAm graft polymerization}

The surface morphology of BOPP before and after AAm graft polymerization was observed using the SEM. Figure 4.49 shows the SEM images of virgin, ozonated, and AAm-grafted PP film at 5000x magnification. It was observed in Figures $4.49 \mathrm{~b}$ and $4.49 \mathrm{c}$ 
(two different areas of the same sample) that the AAm graft covered the entire surface of the BOPP film after 45 minutes of the aqueous phase ozonation induced reaction; while in Figures $4.49 \mathrm{~d}$ and $4.49 \mathrm{e}$ (two different areas of the same sample), only points of the AAm graft were observed on the BOPP film after 45 minutes of the gaseous phase ozonation induced reaction. The greater covering of AAm graft polymers indicates that the reaction induced by the aqueous phase ozonation was more effective than that of the gaseous phase counterpart. The images show that radical graft polymerization occurred on the active points and propagated to an irregular shaped mass (images $4.49 \mathrm{~d}$ and $4.49 \mathrm{e}$ ), and grew to a layer that completely covered the film surface when the amounts of active points available and reaction time were sufficient (Figures $4.49 \mathrm{~b}$ and $4.49 \mathrm{c}$ ).

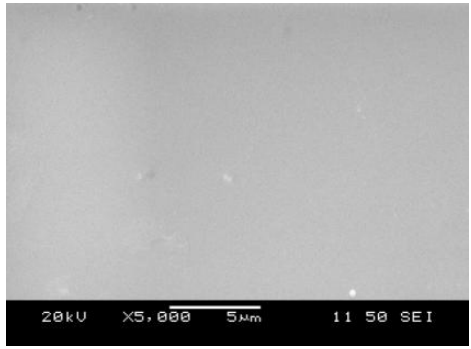

a.

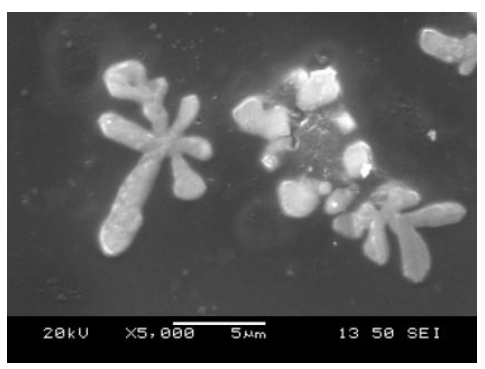

d.

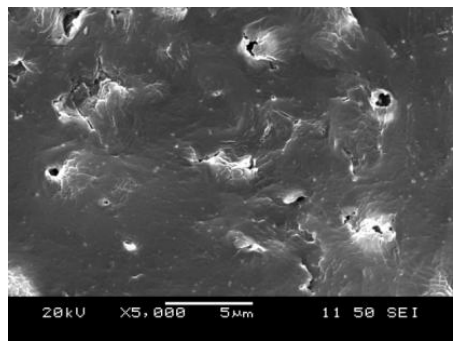

b.

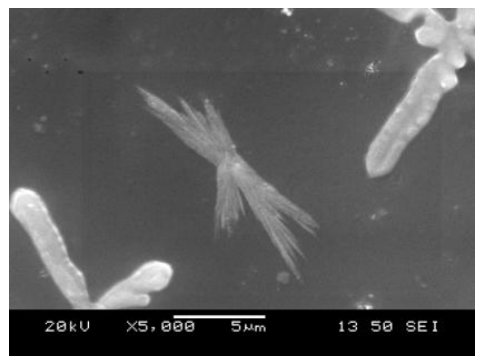

e.

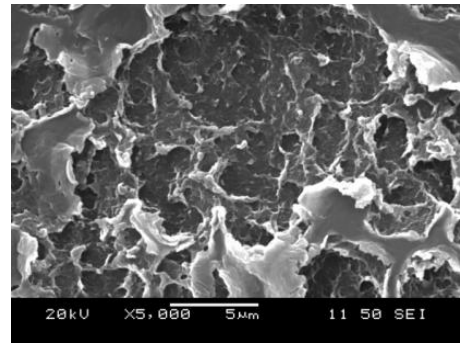

c.

Fig 4.49 SEM images of BOPP grafted with AAm, a. BOPP virgin, b. A45 + AAm graft-1, c. A45 + AAm graft-2, d. G45 + AAm graft-1, and e. G45 + AAm graft-2

\subsubsection{SEM images of the BSA adsorption test on BOPP}

The surface morphology of the protein (BSA) adsorption test on BOPP before and after AAm graft polymerization was observed with SEM. Figure 4.50 shows the SEM images of virgin, gaseous and aqueous phase ozonated, and AAm-grafted BOPP films at 5000x 
magnification. As shown in Images a, b, and c of Figure 4.50, BSA adsorbed on the surface of all of the BOPP films - virgin, G45 + AAm, and A45 + AAm. It was observed that the AAm grafted films had the least adsorption of protein, indicating that the higher the hydrophilicity of the membrane, the less adsorptive it is. The AAm graft polymerization induced by ozonation improved the hydrophilicity and reduced the protein adsorption property.

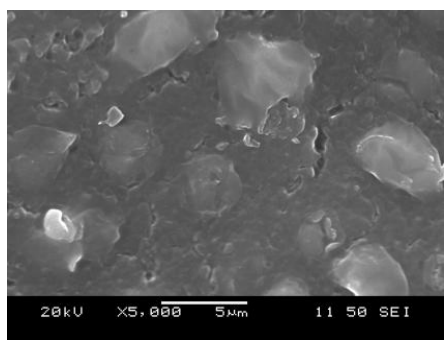

$a$.

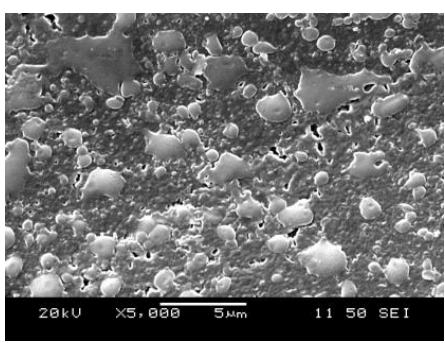

$b$.

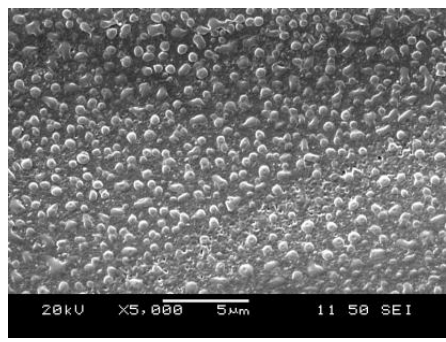

c.

Fig 4.50 SEM images of BOPP adsorbed with BSA after grafted with AAm, a. BOPP virgin + BSA, b. G45 + AAm + BSA, and c. A45 + AAm + BSA

The surface morphology of the protein (BSA) adsorption test of BOPP before and after the AAm or PEG grafting was observed using SEM. Figure 4.51 shows the SEM images at 30x magnification. As shown in Images a, b, c, and d of Figure 4.51, BSA adsorbed on the surfaces of AAm, PEG200, PEG600 and PEG3350 grafted BOPP. It was further observed that the PEG3350 grafted film had the least adsorption of protein on the surface.

From results of the filtration of PPMM membrane modified by PEG grafting, as shown in Figure 4.44, the BSA permeate rate of PEG200, PEG600 and PEG3350 grafted membranes was improved compared to that of the virgin membrane. The PEG200 grafted PPMM had the largest permeate flow rate, which indicated that the PEG200 had the best access to graft on membrane surface and pores. The filtration result is different from that of absorption, while PEG3350 grafted PPMM showed a less protein absorbed on the surface. It is possible that the size of PEG3350 hindered its grafting into the membrane surfaces and pores, therefore, the PPMM grafted with PEG3350 does not have the hydrophilicity as good as that grafted with PEG200. Although the grafting of PEG3350 was not as good as PEG200 
on the PPMM surface, the larger size of molecule could exclude more absorption of protein.

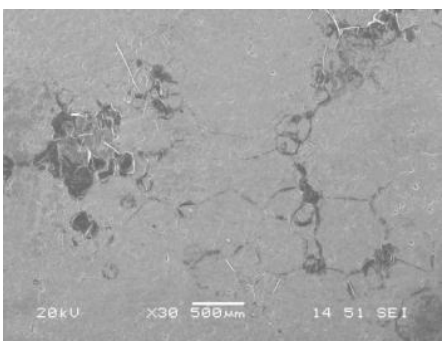

a.

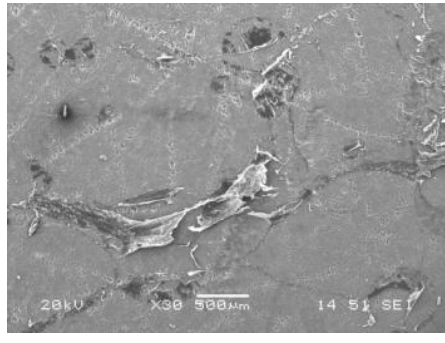

b.

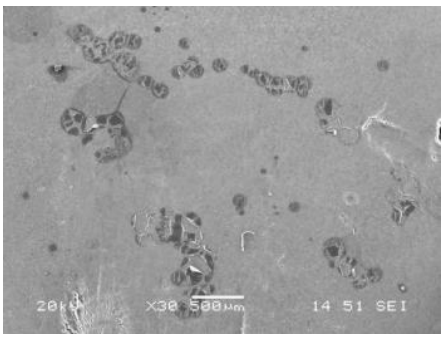

c.

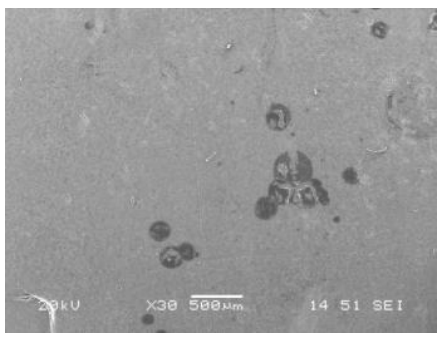

d.

Fig 4.51 SEM images of PPMM adsorbed with BSA after grafted with AAm and PEG, a. PPMM A30 + AAm - BSA soaked, $b$. PPMM A30 + PEG200 - BSA soaked, c. PPMM A30 + PEG600 - BSA soaked, and d. PPMM A30 + PEG3350 - BSA soaked

\subsection{Discussion of the Mechanism and Reaction Models}

The mechanism and reaction models of ozonation, ozonation with scavengers, catalytic ozonation, graft polymerization, filtration flux and fouling are discussed in the following sections.

\subsubsection{Kinetics of ozonation of membrane and film surfaces}

The ozonation process in aqueous phase consists of direct and indirect reactions which can occur at the same time. An oxidation model of compound M can be described as the sum of the two reaction pathways as follows (Gottschalk et al., 2000):

$-\frac{d c(M)}{d t}=\left[\mathrm{k}_{\mathrm{D}} \mathrm{c}\left(\mathrm{O}_{3}\right)+\mathrm{k}_{\mathrm{R}} \mathrm{c}(\mathrm{OH} \cdot)\right] \mathrm{C}(\mathrm{M})$ 
where

M: model compound

$\mathrm{k}_{\mathrm{D}}$ : reaction rate constant for the direct reaction

$\mathrm{k}_{\mathrm{R}}$ : reaction rate constant for the indirect reaction

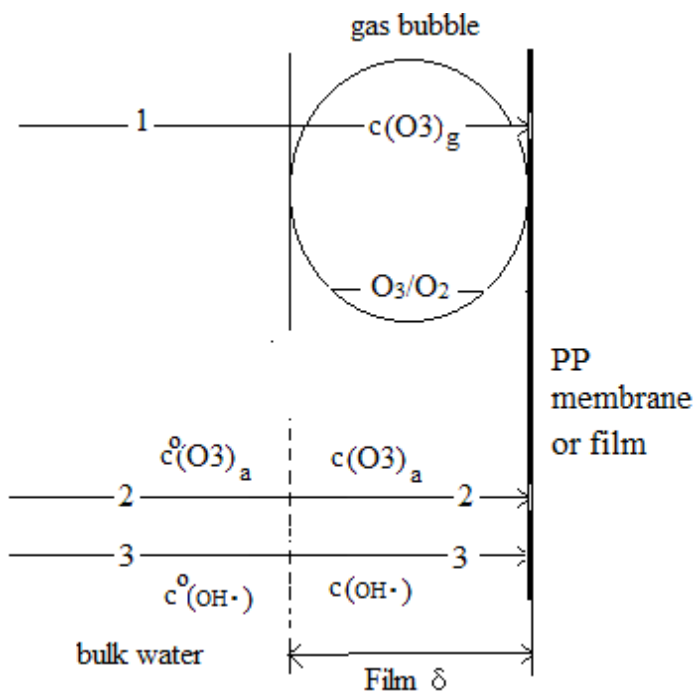

Fig 4.52 Schematic of ozone reacting with PPMM in the aqueous phase

i) The peroxide generation equation. As shown in Figure 4.52, in this research ozone reacts with PPMM/BOPP in the aqueous phase in three ways: 1. Gaseous ozone in gas bubbles $-\mathrm{c}\left(\mathrm{O}_{3}\right)_{\mathrm{g}} ; 2$. Dissolved ozone $-\mathrm{c}\left(\mathrm{O}_{3}\right)_{\mathrm{a}} ; 3 . \mathrm{OH} \cdot$ radicals generated in water $-\mathrm{c}(\mathrm{OH} \cdot)$. $\mathrm{c}\left(\mathrm{O}_{3}\right)_{\mathrm{g}}$ is held constant by controlling the gas feed (assuming the gas bubbles hit and leave the surfaces of PPMM at a constant rate). $\mathrm{c}\left(\mathrm{O}_{3}\right)_{\mathrm{a}}$ and $\mathrm{c}(\mathrm{OH} \cdot)$ are related to ozone dissolving and decomposition.

For $\mathrm{RH}+\mathrm{O}_{3} \rightarrow \mathrm{ROOH}$ in the aqueous phase reaction in this research, it was assumed that the gaseous ozone, dissolved ozone, and generated $\mathrm{OH} \cdot$ all contributed to the peroxide generation. The ozonation of polypropylene membrane and films can be described as:

$\frac{d c(P e r)}{d t}=\alpha\left(-\frac{d c(M)}{d t}\right)=\alpha c(\mathrm{M})\left[\mathrm{k}_{\mathrm{g}} \mathrm{c}\left(\mathrm{O}_{3}\right)_{\mathrm{g}}+\mathrm{k}_{\mathrm{a}} \mathrm{c}\left(\mathrm{O}_{3}\right)_{\mathrm{a}}+\mathrm{k}_{\mathrm{R}} \mathrm{c}(\mathrm{OH} \cdot)\right]$ 
where $c(P e r)$ is the concentration of generated peroxide; $\mathrm{k}_{\mathrm{g}}, \mathrm{k}_{\mathrm{a}}$, and $\mathrm{k}_{\mathrm{R}}$ are the reaction constants of gas ozone, molecular ozone in water, and hydroxyl radicals, respectively; $\alpha$ is a constant; and $\mathrm{c}(\mathrm{M})$ is the reactive molecule concentration of the PPMM \& BOPP surface, which is related to the material of the sample pieces.

ii) Ozone concentration in a non reaction gas-liquid system. Henry's Law is stated as:

$x_{A}=\frac{P_{A}}{H}$

where

$x_{\mathrm{A}}$ : moles solute $-\mathrm{A} /$ mole solution

$P_{A}$ : partial pressure of $\mathrm{A}$

$H$ : Henry's Law Constant

Interpreting this formula for gaseous ozone dissolved (ozone solubility) in water gives,

$\mathrm{c} *\left(\mathrm{O}_{3}\right)_{\mathrm{a}}=\frac{P\left(O_{3}\right)_{g}}{H}$

Assume ozone is an ideal gas, and according to the Ideal Gas Law:

$P\left(O_{3}\right)_{g}=R T\left(O_{3}\right)_{g}$

Combination of Equation (4-8) and (4-9) gives,

$\mathrm{c} *\left(\mathrm{O}_{3}\right)_{\mathrm{a}}=\frac{R T}{H} c\left(O_{3}\right)_{g}$

where $\mathrm{R}$ is the Ideal Gas Constant, $\mathrm{T}$ is the temperature in $\mathrm{K}$.

iii) Ozone concentration in a gas-liquid system with reaction. Refer to Equation 
(2-16) in Section 2.3.2 "Decomposition of ozone in water and pH effect", when the ozone gas mixture $\left(\mathrm{O}_{2}\right.$ and $\left.\mathrm{O}_{3}\right)$ is continuously fed into the reactor, assume the gas and water phases are perfectly mixed, according to the hypothesis of perfect mixing, a molar balance of ozone in the water leads to the following equation (Beltran, 2004):

$\frac{d c\left(O_{3}\right)_{a}}{d t}=k_{L a}\left[c^{*}\left(O_{3}\right)_{a}-c\left(O_{3}\right)_{a}\right]-k_{1} c\left(O_{3}\right)_{a}$

where $k_{L a}$ and $k_{l}$ are the volumetric mass-transfer coefficient through the water phase and the rate constant of the ozone reaction (in pure water, the reaction is decomposition), respectively, $\mathrm{c}^{*}\left(\mathrm{O}_{3}\right)_{\mathrm{a}}$ is the ozone solubility, also is the interface concentration.

In a well-agitated tank, the concentration of dissolved ozone increases with time until it reaches a stationary value, at this time, $\frac{d c\left(O_{3}\right)_{a}}{d t}=0$, thus

$k_{L a}\left[c^{*}\left(O_{3}\right)_{a}-c\left(O_{3}\right)_{a}\right]-k_{1} c\left(O_{3}\right)_{a}=0$

Therefore

$c\left(O_{3}\right)_{a}=\frac{k_{L a} c^{*}\left(O_{3}\right)_{a}}{\left(k_{L a}+k_{1}\right)}$

Combination of Equation (4-10) with Equation (4-12) gives,

$c\left(O_{3}\right)_{a}=\frac{k_{L a} R T}{H\left(k_{L a}+k_{1}\right)} c\left(O_{3}\right)_{g}=\beta c\left(O_{3}\right)_{g}$

$\beta=\frac{k_{L a} R T}{H\left(k_{L a}+k_{1}\right)}$ is constant when the ozone gas is fed in continuously at constant temperature. 
iv) Hydroxyl radical concentration in the aqueous solution. As shown in Equations (2-6)-(2-9) (Beltran, 2004) and Equations (2-38)-(2-42) (Staehelln et al., 1982), the principal reactions of $\mathrm{O}_{3}$ in water to generate hydroxyl radicals are as follows:

$\mathrm{O}_{3}+\mathrm{OH}^{-} \longrightarrow \mathrm{HO}_{2}^{-}+\mathrm{O}_{2}$

$\mathrm{HO}_{2}^{-}+\mathrm{O}_{3} \longrightarrow \cdot \mathrm{O}_{2}^{-}+\mathrm{OH} \cdot+\mathrm{O}_{2}$

$\cdot \mathrm{O}_{2}^{-}+\mathrm{O}_{3} \longrightarrow \cdot \mathrm{O}_{3}{ }^{-}+\mathrm{O}_{2}$

$\cdot \mathrm{O}_{3}{ }^{-}+\mathrm{H}_{2} \mathrm{O} \longrightarrow \mathrm{HO}^{-}+\mathrm{OH}^{\cdot}+\mathrm{O}_{2}$

To sum up the principal reactions, $\mathrm{OH} \cdot$ was generated by the following reaction

$3 \mathrm{O}_{3}+\mathrm{H}_{2} \mathrm{O} \stackrel{\mathrm{k}}{\longrightarrow} \quad 2 \mathrm{OH} \cdot+4 \mathrm{O}_{2}$

while $\mathrm{OH} \cdot$ was consumed by the following paths

$\begin{array}{lll}\mathrm{OH} \cdot+\mathrm{R}-\mathrm{H} & \stackrel{k_{d} \rightarrow}{\longrightarrow} \mathrm{R} \cdot+\mathrm{H}_{2} \mathrm{O} \\ \mathrm{OH} \cdot+\mathrm{S} & \stackrel{k_{s}}{\longrightarrow} & \text { inactive molecules }\end{array}$

where $\mathrm{R}-\mathrm{H}$ is the substrate used to generate peroxide, and $\mathrm{S}$ is the scavenger or radical terminator, $\mathrm{k}_{\mathrm{d}}$ is the reactive constant of direct reaction, $\mathrm{k}_{\mathrm{s}}$ is the reactive constant of hydroxyl radicals with scavenger or terminator. $R \cdot$ will react continually with ozone, oxygen and the polymer molecules to obtain oxygen and hydrogen, and reconstruct to peroxide.

The generated and eliminated $\mathrm{OH} \cdot$ were in equilibrium:

$$
\begin{gathered}
k_{d} \mathrm{c}(\mathrm{OH} \cdot) \mathrm{c}(\mathrm{M})+k_{s} \mathrm{c}(\mathrm{OH} \cdot) \mathrm{c}(\mathrm{S})=\frac{2}{3} k_{O H} \mathrm{c}\left(\mathrm{O}_{3}\right)_{\mathrm{a}} \\
\mathrm{c}(\mathrm{OH} \cdot)=\frac{2 k_{O H}}{3\left[k_{d} c(M)+k_{s} c(S)\right]} \quad \mathrm{c}\left(\mathrm{O}_{3}\right)_{\mathrm{a}}
\end{gathered}
$$


The scavenger in the system is negligible:

$\mathrm{c}(\mathrm{OH} \cdot) \approx \frac{2 k_{O H}}{3 k_{d} c(M)} \quad \mathrm{c}\left(\mathrm{O}_{3}\right)_{\mathrm{a}}$

The combination of Equations (4-13) and (4-14) gives,

$\mathrm{c}(\mathrm{OH} \cdot)=\frac{2 k_{O H} \beta}{3 k_{d} c(M)} C\left(O_{3}\right)_{g}$

Combination of Equations (4-7), (4-13), and (4-15), and the oxidation kinetics can be described as:

$$
\begin{aligned}
& \frac{d c(M)}{d t}=-\mathrm{c}(\mathrm{M})\left[k_{g} \mathrm{c}\left(\mathrm{O}_{3}\right)_{\mathrm{g}}+k_{a} \mathrm{c}\left(\mathrm{O}_{3}\right)_{\mathrm{a}}+k_{R} \mathrm{c}(\mathrm{OH} \cdot)\right] \\
& =-\mathrm{c}(\mathrm{M})\left[k_{g} \mathrm{c}\left(\mathrm{O}_{3}\right)_{\mathrm{g}}+k_{a} \beta C\left(O_{3}\right)_{g}+k_{R} \frac{2 k_{O H} \beta}{3 k_{d} c(M)} C\left(O_{3}\right)_{g}\right] \\
& =-\left[\left(k_{g}+k_{a} \beta+k_{R} \frac{2 k_{O H}}{3 k_{d}} \beta\right)\right] \mathrm{c}\left(\mathrm{O}_{3}\right)_{\mathrm{g}} \mathrm{c}(\mathrm{M})
\end{aligned}
$$

For PPMM ozonation when there is excess ozone with constantly feeding in:

$$
\frac{d c(M)}{d t}=-k_{M} \mathrm{c}(\mathrm{M})
$$

where $k_{M}=\left(k_{g}+k_{a} \beta+k_{R} \frac{2 k_{O H}}{3 k_{d}} \beta\right) \mathrm{c}\left(\mathrm{O}_{3}\right)_{\mathrm{g}}$

Integration of Equation (4-17) yields, 


$$
\frac{c(M)}{c\left(M_{o}\right)}=\exp \left(-k_{M} t\right)
$$

where $\mathrm{c}\left(\mathrm{M}_{\mathrm{o}}\right)$ is the active molecular concentration of polymer at beginning

$$
\mathrm{c}(\mathrm{M})=\mathrm{c}\left(\mathrm{M}_{\mathrm{o}}\right) \exp \left(-k_{M} t\right)
$$

Combination of Equations (4-7), (4-17), and (4-18) gives the ozonation model as:

$$
\frac{d c(P e r)}{d t}=\alpha\left[-\frac{d c(M)}{d t}\right]=\alpha k_{M} \mathrm{c}(\mathrm{M})=a k_{M} \mathrm{c}\left(\mathrm{M}_{\mathrm{o}}\right) \exp \left(-k_{M} t\right)
$$

Integration of the above equation gives, $\int_{0}^{c(\text { per })} d c($ Per $)=\int_{0}^{t}\left[\alpha k_{M} c\left(M_{o}\right) \exp \left(-k_{M} t\right)\right] d t$

$\mathrm{c}(\operatorname{Per})=\alpha c\left(M_{o}\right)\left[1-\exp \left(-k_{M} \mathrm{t}\right)\right]$

This is the mathematical model of the aqueous phase ozonation of PPMM, which is an exponential relationship between the peroxide concentration and ozonation time. Comparing this equation to Equation 2.49 that was proposed by Razumovskii \& Kefeli from their investigation of the mechanism of gaseous ozonation of polyethylene powder in 1971, they are in agreement, only the constant $k_{M}$ and $\alpha$ are different. Figure 4.1 showed a relationship of PPMM in the aqueous phase ozonation of $c($ Per $)=2.7548(1-\exp (-0.0081 \mathrm{t}))$, thus $\alpha c\left(M_{o}\right)=2.7548, k_{M}=0.0081$; Figure 4.25 showed a relationship of BOPP in the aqueous phase ozonation of $\mathrm{c}(\operatorname{Per})=0.2462(1-\exp (-0.02035 \mathrm{t}))$, thus $\alpha c\left(M_{o}\right)=0.2462, k_{M}=0.02035$.

v) In the gaseous phase ozonation,

$$
\frac{d c(P e r)}{d t}=a^{\prime}\left[-\frac{d c(M)}{d t}\right]=\alpha^{\prime} c(\mathrm{M}) k_{g} \mathrm{c}\left(\mathrm{O}_{3}\right)_{\mathrm{g}}
$$


When ozone is in excess $\frac{d c(M)}{d t}=-k_{g} \mathrm{c}\left(\mathrm{O}_{3}\right)_{\mathrm{g}} \mathrm{c}(\mathrm{M})=-k_{M}{ }^{\prime} \mathrm{c}(\mathrm{M})$

where $k_{M}{ }^{\prime}=k_{g} \mathrm{c}\left(\mathrm{O}_{3}\right)_{\mathrm{g}}$

Integration of Equation (4-21) gives

$\mathrm{c}(\mathrm{M})=\mathrm{c}\left(\mathrm{M}_{\mathrm{o}}\right) \exp \left(-k_{M}{ }^{\prime} t\right)$

Combination of Equation (4-20) and (4-21) yields,

$\frac{d c(P e r)}{d t}=-\alpha^{\prime} k_{M}^{\prime} \quad c\left(M_{o}\right) \exp \left(-k_{M}^{\prime} t\right)$

Integration of above equation gives

$\int_{0}^{c(p e r)} d c($ Per $)=\int_{0}^{t}\left[-\alpha^{\prime} k_{M}{ }^{\prime} c\left(M_{o}\right) \exp \left(-k_{M}{ }^{\prime} t\right)\right] d t$

$\mathrm{c}(\mathrm{Per})=\mathrm{a}^{\prime} \mathrm{c}\left(\mathrm{M}_{\mathrm{o}}\right)\left[1-\exp \left(-k^{\prime}{ }_{M} \mathrm{t}\right)\right]$

This is the mathematical model of the gaseous phase ozonation, which also represents the relationship of the peroxide concentration with ozonation time, as shown in Figure 4.1 and Figure 4.25. Figure 4.1 showed a relationship of PPMM in the gaseous phase ozonation of $\mathrm{c}(\operatorname{Per})=5.369(1-\exp (-0.0041 \mathrm{t}))$, thus $\alpha^{\prime} c\left(M_{o}\right)=5.369, k_{M}{ }^{\prime}=0.0041$; Figure 4.25 showed a relationship of BOPP in the aqueous phase ozonation of $c(\operatorname{Per})=0.2800(1-\exp (-0.01738 t))$, thus $\alpha^{\prime} c\left(M_{o}\right)=0.2800, k_{M}^{\prime}=0.01738$.

\subsubsection{Scavengers and the mechanism}

Scavenger is a material that captures the free radicals. Carbonates usually acted as a scavenger (Beltran, 2004), due to the following reactions: 


$$
\begin{aligned}
& \mathrm{HCO}_{3}{ }^{-}+\mathrm{HO} \cdot \stackrel{k_{c 1}=8.5 \times 10^{6} M^{-1} s^{-1}}{\longrightarrow} \mathrm{CO}_{3}{ }^{-}+\mathrm{H}_{2} \mathrm{O} \\
& \mathrm{CO}_{3}{ }^{2-}+\mathrm{HO} \cdot \quad \stackrel{k_{c 2}=3.9 \times 10^{8} M^{-1} s^{-1}}{\longrightarrow} \mathrm{CO}_{3}{ }^{-}+\mathrm{OH}^{-}
\end{aligned}
$$

In this study, freshly made distilled water (DW) was used, the $\mathrm{pH}$ was tested and maintained at 5.4, and DW was purged by the oxygen and ozone gas mixture for a certain time before the sample's ozonation started. This method removed carbon dioxide dissolved in DW, which acted as scavenger:

$\mathrm{CO}_{2}+\mathrm{H}_{2} \mathrm{O} \longleftrightarrow \mathrm{H}_{2} \mathrm{CO}_{3} \longleftrightarrow \mathrm{HCO}_{3}{ }^{-}+\mathrm{H}^{+} \longleftrightarrow \mathrm{CO}_{3}{ }^{-}+2 \mathrm{H}^{+}$

Tert-Butanol is another material acted as scavenger:<smiles>CC(C)(C)O[CH+]OC(C)(C)C</smiles>

Polypropylene $(\mathrm{RH})$ reacts with ozone and with the scavenger as shown in Figure 4.53. Ozone reacts with $\mathrm{RH}$, creating $\mathrm{ROOH}, \mathrm{R} \cdot$, and $\mathrm{OH} \cdot$. $\mathrm{ROOH}$ broke into $\mathrm{R} \cdot$ and $\mathrm{OH} \cdot$ when heated, and induced radical polymerization. $\mathrm{OH} \cdot$ can react with $\mathrm{RH}$ to create $\mathrm{R} \cdot$, or can be eliminated by a scavenger. 


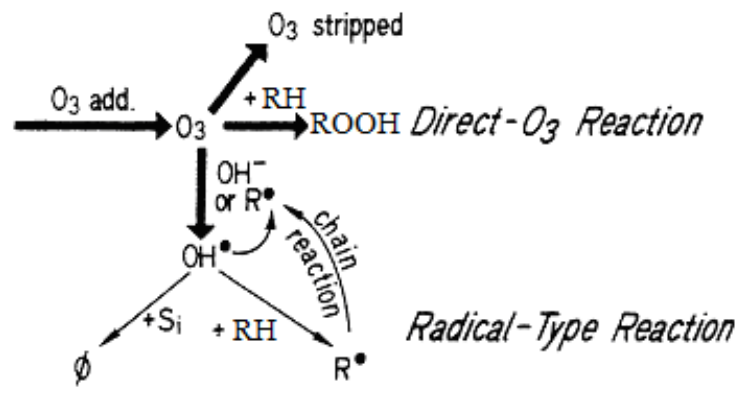

Fig 4.53 The two types of reactions of ozone in aqueous solution. $\mathrm{RH}(\mathrm{PP})$ reacts with $\mathrm{O}_{3}$ and $\mathrm{OH} \cdot$ yielding, respectively, $\mathrm{ROOH}$ and the radicals $\mathrm{R} \cdot \mathrm{S}_{\mathrm{i}}$ (scavenger) captures $\mathrm{OH} \cdot$ radicals yielding the byproduct $\Phi$ (Beltran, 2004)

Scavengers captured all the radicals generated in the ozonation solution; therefore, the ozonation model of polypropylene membrane and films (Equation 4-7) becomes:

$\frac{d c(P e r)}{d t}=\alpha^{\prime \prime}\left[-\frac{d c(M)}{d t}\right]=\alpha^{\prime \prime} \mathrm{c}(\mathrm{M})\left[k_{g} \mathrm{c}\left(\mathrm{O}_{3}\right)_{\mathrm{g}}+k_{a} \mathrm{c}\left(\mathrm{O}_{3}\right)_{\mathrm{a}}\right]$

Combination of Equations (4-13) and (4-28) gives,

$$
\begin{aligned}
& \frac{d c(M)}{d t}=-\mathrm{c}(\mathrm{M})\left[k_{g} \mathrm{c}\left(\mathrm{O}_{3}\right)_{\mathrm{g}}+k_{a} \beta \mathrm{c}\left(\mathrm{O}_{3}\right)_{\mathrm{g}}\right] \\
& =-\left(k_{g}+k_{a} \beta\right) \mathrm{c}\left(\mathrm{O}_{3}\right)_{\mathrm{g}} \mathrm{c}(\mathrm{M})
\end{aligned}
$$

Define $k_{M}{ }^{\prime \prime}=-\left(k_{g}+k_{a} \beta\right) \mathrm{c}\left(\mathrm{O}_{3}\right)_{\mathrm{g}}$

$$
\frac{d c(M)}{d t}=-k_{M}{ }^{\prime \prime} \mathrm{c}(\mathrm{M})
$$

Integration of Equation (4-30) yields, 
$\mathrm{c}(\mathrm{M})=\mathrm{c}\left(\mathrm{M}_{\mathrm{o}}\right) \exp \left(-k_{M}{ }^{\prime \prime}\right)$

Combination of Equations (4-28), (4-30) and (4-31), and integration gives,

$\mathrm{c}($ Per $)=\alpha^{\prime \prime} \mathrm{c}\left(\mathrm{M}_{\mathrm{o}}\right)\left[1-\exp \left(-k_{M} \mathrm{t}\right)\right]$

This is the mathematical model of the aqueous phase ozonation with scavenger. Figure 4.8 showed a relationship of PPMM in the aqueous phase ozonation with scavenger $\mathrm{Na}_{2} \mathrm{CO}_{3}$ of $c($ Per $)=1.2922(1-\exp (-0.0189 \mathrm{t}))$, thus $\alpha^{\prime \prime} c\left(M_{o}\right)=1.2922, k_{M}{ }^{\prime \prime}=0.0189$; Figure 4.25 showed a relationship of BOPP in the aqueous phase ozonation with scavenger $\mathrm{Na}_{2} \mathrm{CO}_{3}$ of $\mathrm{c}($ Per $)=0.2569(1-\exp (-0.0140 \mathrm{t}))$, thus $\alpha^{\prime \prime} c\left(M_{o}\right)=0.2569, k_{M}{ }^{\prime \prime}=0.0140$.

\subsubsection{The catalytic pathways}

\subsubsection{The $\mathrm{Cu}^{2+}$ catalyst mechanism}

Since there was no information about the mechanism of copper (II) catalyzed ozonation found in the literature, special tests were designed and conducted, and are discussed in Section 4.18.1 to Section 4.18.5 of this thesis, in which the author investigates the mechanism of copper (II) catalyzed ozonation from two perspectives: how the catalysis worked and why there was an optimum concentration of copper (II) cation.

The test results demonstrated the $\mathrm{Cu}^{2+}$ boosted production of radicals and an optimal $\mathrm{Cu}^{2+}$ concentration present. It was also concluded that excess anion $\mathrm{SO}_{4}{ }^{2-}$ would have negatively affected the reaction.

Based on our experimental results, two possible catalytic ozonation pathways are proposed.

Pathway I: $\mathrm{Cu}$ (II) cation reacted with ozone to generate the intermediates $\mathrm{CuOH}^{2+} \bullet$, $\mathrm{HO} \bullet$, and $\mathrm{HO}_{2} \bullet$ radicals (Hill, 1948), and these radicals accelerated the peroxide generation on the PP surface, as shown in Equations (4-33), (4-34), and (4-35). The radical generation was demonstrated in Section 4.1.8.5. Figure 4.54 shows the schematic that the radicals generated by $\mathrm{Cu}^{2+}$ catalyzed ozonation reacted with polypropylene and formed peroxide. 
$\mathrm{Cu}^{2+}+\mathrm{O}_{3}+\mathrm{H}_{2} \mathrm{O} \longrightarrow \mathrm{CuOH}^{2+} \cdot+\mathrm{O}_{2}+\mathrm{HO} \cdot$

$\mathrm{HO} \cdot+\mathrm{O}_{3} \longrightarrow \mathrm{HO}_{2} \cdot+\mathrm{O}_{2}$

$\mathrm{CuOH}^{2+} \cdot+\mathrm{HO}_{2} \cdot \longrightarrow \mathrm{Cu}^{2+}+\mathrm{O}_{2}+\mathrm{H}_{2} \mathrm{O}$

Since the enhanced radicals were demonstrated to be the pathway of catalytic ozonation, the catalytic ozonation of polypropylene membrane and films can be described as:

$\frac{d c(P e r)}{d t}=\alpha\left(-\frac{d c(M)}{d t}\right)=\alpha \mathrm{c}(\mathrm{M})\left[k_{g} \mathrm{c}\left(\mathrm{O}_{3}\right)_{\mathrm{g}}+k_{a} \mathrm{c}\left(\mathrm{O}_{3}\right)_{\mathrm{a}}+k_{R}{ }^{\prime} \mathrm{c}(\mathrm{OH} \cdot)\right]$

where $k_{R}{ }^{\prime}$ is a constant that all radicals contribute to the peroxide generation.

The final equation can be derived as:

$\mathrm{c}(\operatorname{Per})=\alpha c\left(M_{o}\right)\left[1-\exp \left(-k_{M c} \mathrm{t}\right)\right]$

where $k_{M c}=\left(k_{g}+k_{a} \beta+k_{R}{ }^{\prime} \frac{2 k_{O H}}{3 k_{d}} \beta\right) \mathrm{c}\left(\mathrm{O}_{3}\right)_{\mathrm{g}}$

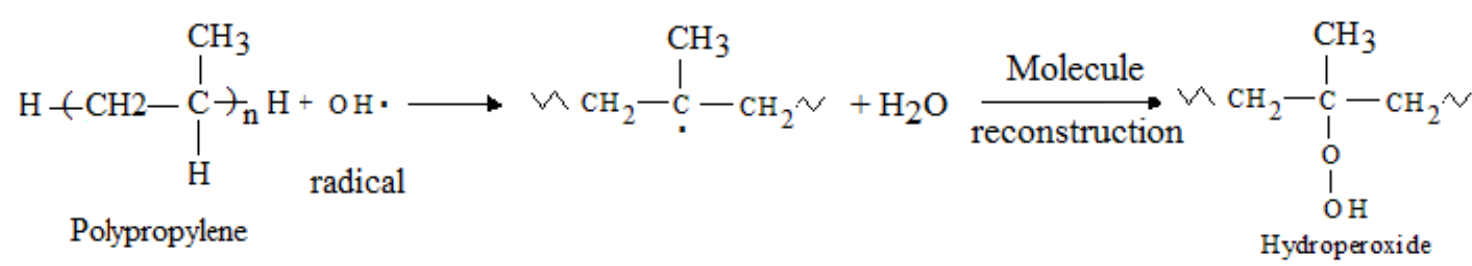

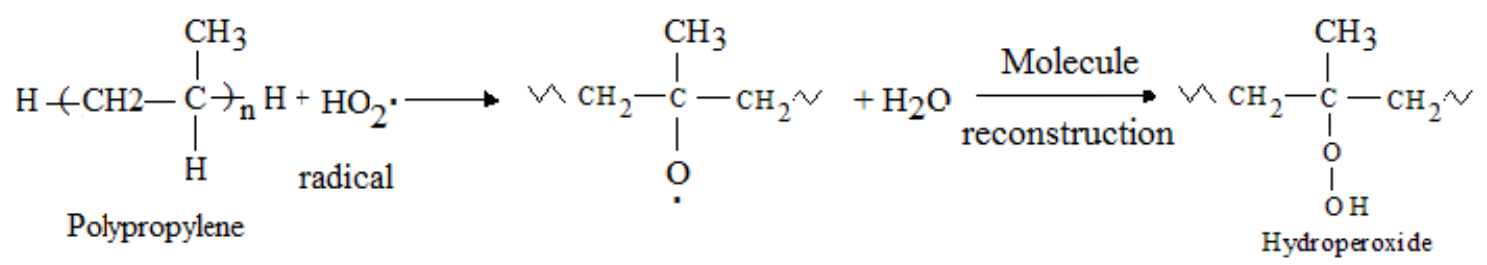

Fig 4.54 Peroxide generation accelerated by hydroxyl radicals 
Pathway II: The $\mathrm{Cu}$ (II) cation combined with oxygen to create the third body $\mathrm{CuO}^{2+}$ and oxygen radical $\mathrm{O}_{2} \cdot \mathrm{CuO}^{2+}$ reacted with ozone to generate two other oxygen radicals. The mechanism is assumed to be similar to that in Pathway I, as discussed in next section, Section 4.6.3.2. These radicals accelerated the peroxide generation on the polypropylene surfaces, as shown in Figure 4.55.

While the hydroxyl radicals in $\mathrm{Cu}^{2+}$ catalytic ozonation could have been affected by anions such as $\mathrm{PO}_{4}{ }^{3-}$ and $\mathrm{CO}_{3}{ }^{2-}$ (Beltran F., 2004), $\mathrm{SO}_{4}{ }^{2-}$ is assumed to have the following function:

$\mathrm{HO} \cdot+\mathrm{SO}_{4}^{2-} \rightarrow \mathrm{OH}^{-}+\mathrm{SO}_{4}^{\cdot}$

$\mathrm{SO}_{4}{ }^{-}$could not help the peroxide creation; thus, $\mathrm{SO}_{4}{ }^{2-}$ had a negative effect on the ozonation. The tests in Figures 4.16 and 4.17 demonstrate this phenomenon. The $\mathrm{Cu}^{2+}$ catalytic ozonation had an optimal concentration; Figure 4.11, Figure 4.18 and Figure 4.19 indicate that this optimum concentration of $\mathrm{Cu}^{2+}$ was $0.787 \mathrm{mmol} / \mathrm{L}$.

At $20^{\circ} \mathrm{C}$ ozone solubility is $12.86 \mathrm{mg} / \mathrm{L}$ (Table 2.3 ) in water. The actual concentration, obtained by the standard indigo analysis as shown in Table 4.1, was a little lower, at 9-10 $\mathrm{mg} / \mathrm{L}$ at $22^{\circ} \mathrm{C}$, and the number of ozone molecules of $9.12 \mathrm{mg} / \mathrm{L}$ is $1.144 \times 10^{20}$ (corresponding to the Avogadro constant, mole molecule number is $6.022 \times 20^{23}$ ); while the number of $\mathrm{Cu}^{2+}$ ions at $0.787 \mathrm{mmol} / \mathrm{L}$ is $4.74 \times 10^{20}$, which is also the number of $\mathrm{SO}_{4}{ }^{2-}$ ions. The best catalytic results were achieved at this ratio (by the decomposition of ozone to $\cdot \mathrm{OH}$ radicals) in which the number of $\mathrm{Cu}^{2+}$ ions was almost 4 times that of ozone molecules. When the $\mathrm{CuSO}_{4} \cdot 5 \mathrm{H}_{2} \mathrm{O}$ concentration was increased, the increased $\mathrm{Cu}^{2+}$ could not help generate more peroxide, while the increased $\mathrm{SO}_{4}{ }^{2-}$ consumed the generated $\cdot \mathrm{OH}$ radicals. On the other hand, when the $\mathrm{CuSO}_{4} \cdot 5 \mathrm{H}_{2} \mathrm{O}$ concentration was decreased, the concentration of $\mathrm{Cu}^{2+}$ was not sufficient. Therefore, the optimum concentration of $\mathrm{Cu}^{2+}$ catalyst was shown at $0.787 \mathrm{mmol} / \mathrm{L}$.

In Section 4.1.9, the concentration $0.6160 \mathrm{mmol} / \mathrm{L}$ of $\mathrm{Fe}^{3+}$ was demonstrated to have the best catalytic results; the number of $\mathrm{Fe}^{3+}$ ions per liter is $3.71 \times 10^{20}$, which is in the same grade of molecule number of dissolved ozone (assume the saturated ozone concentration has 
no significant difference between $\mathrm{Fe}^{3+}$ catalyzed solution and $\mathrm{Cu}^{2+}$ catalyzed solution). The number of $\mathrm{Fe}^{3+}$ ions was almost 3.2 times that of ozone molecules, and the best catalytic results were achieved by decomposing ozone to $\cdot \mathrm{OH}$ radicals.

\subsubsection{Fe(III) catalyst}

The following reaction mechanisms are proposed pathways for ferric catalyzed ozonation.

Pathway I: The $\mathrm{Fe}^{3+}$ cation constitutes the essential third body $\mathrm{FeO}^{3+}$, the reaction rate being determined by the appropriate collision frequency, in accordance with Calderbank et al., 1976. Ozone decomposition is observed to have a low activation energy in Reaction (4-40), and $\mathrm{O}_{3}$ reacts much more rapidly than $\mathrm{O}_{2}$ does. The activation energy of this reactivation reaction is greater than that of Reaction (4-40). Reactions (4-39) and (4-40) give a total reaction as shown in Equation (4-41), in which a $\mathrm{Fe}(\mathrm{III})$ cation reacts with two $\mathrm{O}_{3}$, generating three $\mathrm{O}_{2} \cdot$ radicals, which accelerate the peroxide generation on the PP surface, as shown in Figure 4.55.
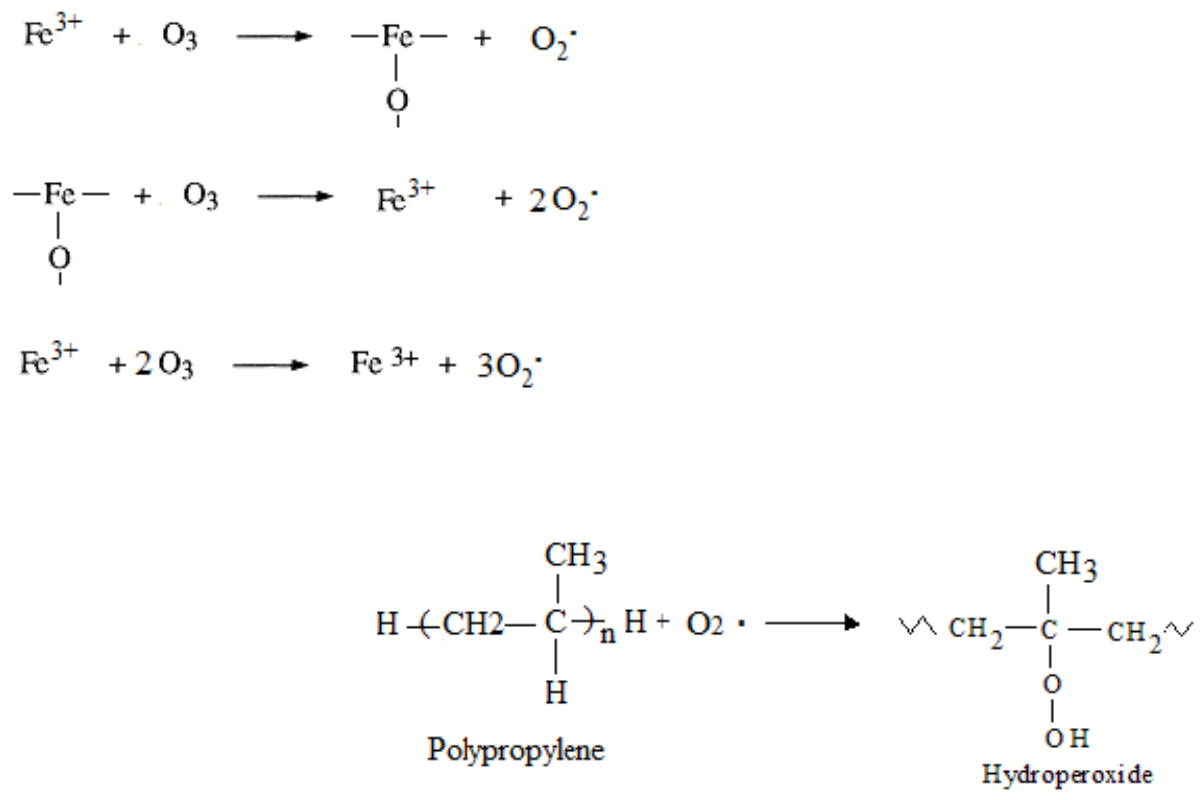

Fig 4.55 Peroxide generation accelerated by oxygen radicals 
Pathway II: $\mathrm{Fe}(\mathrm{III})$ cations reacted with ozone to generate intermediate $\mathrm{FeOH}^{3+}$, $\mathrm{HO}$. radicals and $\mathrm{HO}_{2} \cdot$ radicals. The mechanism was similar to that of Pathway II in Equations (4-33), (4-34), and (4-35) in Section 4.6.3.1, in which these radicals accelerated the peroxide generation on PP surface, as shown in Figure 4.54. 


\section{Chapter 5}

\section{Conclusions}

The following are the conclusions of this work:

a) The results of ozonation of both polypropylene microfiltration membranes (PPMM) and commercial biaxial oriented polypropylene (BOPP) films, show that the concentration of peroxides generated on the membrane and film surfaces increased with the applied ozone dose and ozonation time in both the aqueous and gaseous phases.

b) Free radicals in the aqueous ozonion were found to contribute about $20-30 \%$ to the peroxide generation, the major contribution to peroxide generation coming from the molecule ozone.

c) Heavy metal ions as catalysts were investigated in this research. Copper sulfate hydrate $\left(\mathrm{CuSO}_{4} \cdot 5 \mathrm{H}_{2} \mathrm{O}\right)$ was added as a homogeneous catalyst in the aqueous phase ozonation, and the peroxide generation rate of PPMM was improved by $17 \%$. Fe (III) $\left(\mathrm{FeCl}_{3} \cdot 6 \mathrm{H}_{2} \mathrm{O}\right)$ also was found to have a positive effect on the peroxide generation.

d) Although the generated peroxide concentration of the gaseous phase ozonation was found to be a little higher than that of the aqueous phase counterpart on the substrates, the aqueous phase ozonation generated more monomer-accessible peroxide for subsequent graft polymerization. Different monomers applied to the ozonated membranes and films - AAm, HEMA, and PEG - were all shown to be accessible to the peroxide active sites, and graft polymerization of all these monomers occurred on the substrates, which was proved by FTIR spectrometry, the contact angle test, and SEM images.

e) Hydrophilicity of polypropylene membranes and films was improved by graft polymerization of Acryl amide (AAm), Hydroxyethyl Methacrylate (HEMA), and Polyethylene Glycol (PEG). The hydrophilicity of PPMM was improved by graft polymerization of AAm, HEMA, and PEG initiated by the peroxides, as indicated by the contact angle reduction from $129^{\circ}$ to $91^{\circ}$ of grafted AAm; from $126^{\circ}$ to $74^{\circ}$ of grafted HEMA; and from $126^{\circ}$ to $88^{\circ}$ of grafted PEG. The contact angle of AAm grafted BOPP film 
was reduced from $80^{\circ}$ to $56^{\circ}$. Fourier Transform Infrared (FTIR) spectrometry confirmed the success of the above monomers; and the Scanning Electron Microscope (SEM) images indicated changes in the amorphousity of the graft polymerization. The XRD diffractogram revealed the crystal changes of ozonated and grafted PPMM.

f) The filtration test results demonstrated the improvement of the anti-fouling effect of the modified PPMM by using bovine serum albumin (BSA). The SEM images of the fouled and washed membranes revealed the cake layer fouling and pore blockage, and the recovery of the surface.

g) The mechanisms of the aqueous and gaseous phase ozonation applied to polypropylene membranes and films were discussed. Based on the experimental results, an exponential order of peroxide generation versus time was obtained. The mechanism of the aqueous phase ozonation with scavengers added was discussed as well. Scavengers captured the radicals which generated in the aqueous phase, thus decreasing the peroxide generation speed.

h) The mechanism of the aqueous phase ozonation (employed to PPMM and BOPP) with catalysts $\mathrm{Cu}^{2+}$ and $\mathrm{Fe}^{3+}$ was discussed. A third body $\left(\mathrm{CuOH}^{2+}\right.$ or $\left.\mathrm{FeO}^{3+}\right)$ was postulated to cause intermediate and accelerated $\cdot \mathrm{OH}, \mathrm{HO}_{2} \cdot$ or $\cdot \mathrm{O}_{2}$ radical generation, and thus increase the peroxide generation speed. Special tests were designed and executed to investigate the catalytic mechanism of copper (II) in ozonation. The radical pathway was found in the copper (II) catalyzed ozonation, and the reason for an optimal copper concentration was found.

In general, by the use of homogeneous catalysts, this study further developed the aqueous ozonation into a practical and effective solution to the problem of hydrophobic surfaces of polypropylene (PP) membranes and films. This study also verified experimentally that the PP membranes and films modified in this study had improved hydrophilicity. Moreover, detailed analysis of the reaction mechanisms provided important insights of the polymer surfaces which will contribute significantly to the research of the oxidative modification of polymer surfaces. The results of this study will positively impact the industrial using of PPMM to elongate its duration time of filtration, and improve the applications of BOPP in biomedical areas. 


\section{Nomenclatures and Abbreviates}

AAC

AAm

ATR-IR

BSA

BOPP

CA

$\mathrm{C}_{\mathrm{f}}$

$\mathrm{c}_{\mathrm{L}}^{*}$

$\mathrm{c}(\mathrm{M})$

$\mathrm{c}\left(\mathrm{M}_{\mathrm{o}}\right)$

Cmem

$\mathrm{c}\left(\mathrm{O}_{3}\right)_{\mathrm{g}}$

$\mathrm{c}\left(\mathrm{O}_{3}\right)_{\mathrm{a}}$

$\mathrm{c} *\left(\mathrm{O}_{3}\right)_{\mathrm{a}}$

$C_{f}$

$c_{g}, c_{g i:}$

$c_{L}, c_{L i}$

$\mathrm{c}(\mathrm{OH} \bullet)$

$c($ Per)

$\mathrm{C}_{\text {reten, NOM }}$

Creten,s

$\mathrm{D}^{*}$

$\mathrm{d}_{32}$

DG

$d_{i}$

DI
The interfacial area

Acrylic acid

Acylicamide

Attenuated total reflection infrared

Bovine serum albumin

Biaxial oriented polypropylene film

Cellulose Acetate

Bulk or feed concentration, in weight fraction units

Liquid concentration in equilibrium with the bulk gas concentration

The reactive molecule concentration of PPMM \& BOPP surface

The reactive molecule concentration of PPMM \& BOPP surface at

beginning

Concentration at the membrane surface

Concentration of ozone in gas bulb

Concentration of ozone in water

Ozone solubility

Weight fraction units.

Concentration of gas component in gas mixture

Concentration of gas component in liquid

The concentration of radicals

The generated concentration of peroxide

NOM concentration in the retentate recycle loop

Bulk (retentate recycle loop) salt $(\mathrm{NaCl})$ concentration

Average diameter of the pores

The Sauter diameter

Degree of graft

Diameter of the equivalent disc of each individual bubble

Distilled 
DMAPS N,N'-dimethyl (methacyloyloxyethyl) ammonium propanesulfate

DMAA $\quad N, N$-dimethylacrylamide

DMAEMA Dimethyl aminoethyl methacrylate

DMAPS 3-dimethyl(methacryloyl ethyl)- ammonium propanesulfonate

DPPH 1,1-diphenyl-2-picrylhydrazyl

DMMCA 2-(Dimethylamino) ethyl methacrylate

DW Distilled water

E Enhancement factor for mass transfer with reaction

$\mathrm{E}^{\prime} \quad$ Efficency

EVA Poly(ethylene-co-vinyl alcohol) film

FTIR Fourier transfer infrared

$G_{O_{3}} \quad$ Generation rate term of ozone

GSH Glutathione

h (hr) Hour

$h_{\mathrm{L}} \quad$ Still liquid height

$h_{\mathrm{G}} \quad$ The gas-liquid dispersion height

HAc Glacial acetic acid

HEMA 2-hydroxyethyl methacrylate

IPA Isopropanol

$J \quad$ The relative flux (\%),

$J_{0} \quad$ Initial permeate flux taken at filtration time of $30 \mathrm{~min}$

$\mathrm{J}^{*}\left(\mathrm{~m} \mathrm{~s}^{-1}\right) \quad$ Effective flux associated with back-transport resulting from crossflow

$J_{0, \mathrm{u}} \quad$ The flux before membrane surface modification

$J t \quad$ The permeate flux at filtration time $t$

$J_{T} \quad$ The experimental flux at $T$

$J_{w} \quad$ The volumetric water flux

$\mathrm{k} \quad$ Rate constant of reaction

$\mathrm{k}_{1(\mathrm{~m})} \quad$ Decomposition rate constant

$k_{a} \quad$ Reactive constants of gas ozone 
$\mathrm{K}_{2} \mathrm{Cr}_{2} \mathrm{O}_{7}$

KI

$\mathrm{K}_{\mathrm{La}}$

$\mathrm{k}_{\mathrm{R}}$

LDPE

m

$\mathrm{m}_{\mathrm{r}}$

M

MF

Mpa

MPC

MWCO

$\mathrm{NADPH}$

NaSS

NF

NOM

Nylon
Reaction rate constant for the direct reaction

Reactive constants of molecular ozone in water

Fouling coefficient

Mass transfer constant in aqueous phase

Constants defined by the author to derive the aqueous ozonation formula

Constants defined by the author to derive the gaseous ozonation formula

Constants defined by the author to derive the aqueous ozonation formula with scavenger

Constants defined by the author to derive the catalytic ozonation

Reactive constants of hydroxyl radicals

Reactive constants of hydroxyl radicals with catalyst

Reactive constants of hydroxyl radicals with scavenger

Potassium dichromate

Potassium iodide

Mass transfer constant in gaseous phase

Reaction rate constant for the indirect reaction

Low density polyethylene films

Mass transfer rate

Mass transfer rate with reaction

Model compound

Microfiltration

Tensile strength unit

Methacryloyloxyethyl phosphorylcholine

Molecular weight cut off

Nicotinamide adenine dinucleotide phosphate

Styrene sulfonic acid sodium salt

Nanofiltration

Natural dissolved organic matter

Polyamide film 


\begin{tabular}{|c|c|}
\hline $\mathrm{O}_{3}$ & Ozone \\
\hline OBCS & $O$-butyrylchitosan \\
\hline $\mathrm{O}-\mathrm{C}=\mathrm{O}$ & Carboxyl \\
\hline PA & Polyamide \\
\hline PAN & Polyacylonitrole \\
\hline PE & Polyethylene \\
\hline PES & Polyethersulfone \\
\hline PEG & Poly ethyleneglycol \\
\hline Per & Total peroxide density at time $t$ \\
\hline $\mathrm{Per}^{\circ}$ & Total initial peroxide density; \\
\hline $\operatorname{Per}^{\circ}{ }_{1(\mathrm{~m})}$ & Initial density of the least (most) decomposable peroxide \\
\hline PET & $12 \mu$ biaxial oriented polyethylene terephthalate film \\
\hline PLLA & Poly-L-lactic acid film \\
\hline PMMA & Poly (methyl methacrylate) film \\
\hline PP & Polypropylene \\
\hline PPMM & Polypropylene microfiltration membrane \\
\hline PS & Polystyrene film and powder \\
\hline PSF & Polysulfone film \\
\hline Psi & Pressure unit, pounds per square inch \\
\hline PTFE & Poly (tetrafluoroethylene) film \\
\hline $\mathrm{PVC}$ & Polyvinyl chloride \\
\hline PVP & Polyvinyl pyrolidinone \\
\hline PU & Polyurethance \\
\hline$\Delta \mathrm{P}$ & Pressure different \\
\hline Q & Flow rate of permeate \\
\hline $\mathrm{Q}_{\mathrm{o}}$ & Initiate flow rate of permeate \\
\hline $\mathrm{Ra}$ & Adsorption, biofouling of membrane \\
\hline $\mathrm{Rc}$ & Cake layer fouling of membrane \\
\hline $\mathrm{Rc}, \mathrm{o}$ & Initial resistance of the deposit \\
\hline RH & Represents substrates reacting with ozone \\
\hline
\end{tabular}




\begin{tabular}{|c|c|}
\hline$R_{h}$ & The hydraulic resistance of the membrane \\
\hline$R_{f}$ & The hydraulic resistance under specific (and similar) conditions for all the \\
\hline & solutes in nonmodified and modified \\
\hline $\mathrm{Rm}$ & Membrane resistance \\
\hline Rmem & Intrinsic membrane rejection \\
\hline $\mathrm{Rpb}$ & Pore plugging \\
\hline$r_{O_{3}}$ & Ozone decomposition rate \\
\hline RO & Reverse Osmosis \\
\hline $\mathrm{ROOH}$ & Peroxide/hydroperoxide \\
\hline $\mathrm{S}_{\mathrm{i}}$ & Represents scavenger \\
\hline SEM & Scanning Electronic Microscopy \\
\hline SPEU & Segment Poly (ether-urethane) \\
\hline $\mathrm{t}$ & Time \\
\hline$t_{D}$ & Diffusion time \\
\hline$t_{R}$ & Reaction time \\
\hline $\mathrm{T}$ & Temperature \\
\hline UF & Ultrafiltration \\
\hline Us & The linear rising velocity of an individual bubble \\
\hline$w_{\mathrm{b}}$ & Weight of a membrane before surface modification \\
\hline$w_{\mathrm{a}}$ & Weight of a membrane after surface modification \\
\hline $\mathrm{W}$ & Mass/weight \\
\hline $\mathrm{wt} \%$ & Weight percentage in the gas mixture \\
\hline $\mathrm{X}$ & Ozone uptake \\
\hline
\end{tabular}

\section{Greek letters}

$\alpha$

A ratio defined by the author of proportion of peroxide generation by aqueous ozonation at PPMM or BOPP

$\alpha^{\prime} \quad$ A ratio defined by the author of proportion of peroxide generation by gaseous ozonation at PPMM or BOPP 


\begin{tabular}{|c|c|}
\hline$\alpha ”$ & $\begin{array}{l}\text { A ratio defined by the author of proportion of peroxide generation by } \\
\text { aqueous ozonation with scavengers at PPMM or BOPP }\end{array}$ \\
\hline$\alpha_{\mathrm{b}}$ & Pore blockage parameter \\
\hline$\alpha_{c}$ & Cake formation parameter \\
\hline$\alpha_{\mathrm{s}}$ & A literature correlation \\
\hline$\alpha_{\text {cake }}$ & Specific cake resistance (assumed constant) \\
\hline$\beta$ & Constants defined by the author to derive the aqueous ozonation formula \\
\hline$\beta_{\mathrm{s}}$ & Cmem/Creten, degree of concentration polarization \\
\hline$\sigma_{1 \mathrm{f}}$ & Liquid-fluid interfacial tensions \\
\hline$\sigma_{\mathrm{s}}$ & Osmotic reflection coefficient \\
\hline$\sigma_{\mathrm{sl}}$ & Solid-liquid interfacial tensions \\
\hline$\sigma_{\mathrm{sf}}$ & Solid-fluid interfacial tensions \\
\hline$\delta$ & The radicals' concentration in water when $\mathrm{t}=0$ \\
\hline$\delta_{L 1}, \delta_{L 2}, \delta_{L 3}$ & The film thickness, the characteristic of film theory \\
\hline$\Phi$ & Byproduct \\
\hline$\mu$ & Viscosity \\
\hline$\mu_{0}$ & The viscosity of the permeate \\
\hline$\varepsilon \mathrm{G}$ & The gas hold-up \\
\hline
\end{tabular}

\section{Superindexes}

c

$J$

$\mathrm{k}$

$\min$

Per

Q

S

S

$\mathrm{t}$

Refers to concentration

Refers to the relative flux (\%),

Refers to rate constant of reaction

Refers to minutes

Refers to peroxide

Refers to flow rate

Refers to scavenger

Refers to second

Refers to time 
Refers to temperature

\section{Subindexs}

a

$\mathrm{g}$

1

$*$
Refers to aqueous phase

Refers to gaseous phase

Refers to liquid

Refers to gas-liquid equilibrium conditions 


\section{Appendix I}

\section{Calculation of titration}

The hydrogen peroxide reaction with iodide in acidic medium:

$$
\mathrm{H}_{2} \mathrm{O}_{2}(\mathrm{aq})+2 \mathrm{I}^{-}(\mathrm{aq})+2 \mathrm{H}^{+}(\mathrm{aq}) \longrightarrow \mathrm{I}_{2}(\mathrm{aq})+2 \mathrm{H}_{2} \mathrm{O}(\mathrm{l})
$$

The created $\mathrm{I}_{2}(\mathrm{aq})$ can be investigated by the titration of a standard $\mathrm{S}_{2} \mathrm{O}_{3}{ }^{2-}$ (aq) solution

$$
\mathrm{I}_{2}+2 \mathrm{~S}_{2} \mathrm{O}_{3}^{2-} \longrightarrow 2 \mathrm{I}^{-}+\mathrm{S}_{4} \mathrm{O}_{6}^{2-}
$$

[APHA, 1992, Iodometric Method I, pp 4-38]

We assume the R-OOH has the same function as the $\mathrm{H}_{2} \mathrm{O}_{2}$ in this reaction, according to this formula, the consumption is:

\section{$1 \mathrm{MMOL} \mathrm{R}-\mathrm{OOH}=2 \mathrm{MMOL} \quad \mathrm{S}_{2} \mathrm{O}_{3}{ }^{2-}$ TITRATION CONSUMPTION}

As an example, calculate the peroxide content of HDPE reacting with ozone at $3.7 \% \mathrm{wt}, 1.0 \mathrm{~h}$ in aqueous medium:

Samples: 3 strips of $1.5 " \times 10 "$ HDPE film

Area: $2($ sides $) \times 3($ strips $) \times(1.5$ inch $\times 25.4 \mathrm{~mm}) \times(10$ inch $\times 25.4 \mathrm{~mm})=58064.4 \mathrm{~mm}^{2}$

$$
=0.0580644 \mathrm{~m}^{2}
$$

$0.001 \mathrm{~N}$ standard $\mathrm{Na}_{2} \mathrm{~S}_{2} \mathrm{O}_{3}{ }^{2-}$ (aq) solution titration consumption: $3.6 \mathrm{ml}$

Consumption normality of $\mathrm{S}_{2} \mathrm{O}_{3}{ }^{2-}: 3.6 \mathrm{ml} \times 0.001 \mathrm{~N}=0.0036$

$\mathrm{R}-\mathrm{OOH}$ normality $=$ normality of $\mathrm{S}_{2} \mathrm{O}_{3}{ }^{2-}=0.0036$

Where Normality $=$ molality $\mathrm{x} n$ (where $\mathrm{n}=$ the number of protons exchanged in a reaction) $\mathrm{n}=2$ in this reaction

R-OOH mmol: $0.0036 \div 2=0.0018$

R-OOH (peroxide) density: $0.0018 \mathrm{mmol} \div 0.0580644 \mathrm{~m}^{2}=0.031 \mathrm{mmol} / \mathrm{m}^{2}$ 


\section{Appendix II}

\section{Standard Sodium thiosulfate solution preparation}

$0.1 \mathrm{~N}$ : Dissolve $25.000 \mathrm{~g} \mathrm{Na}_{2} \mathrm{~S}_{2} \mathrm{O}_{3} \cdot 5 \mathrm{H}_{2} \mathrm{O}$ in a 1.000 liter freshly boiled distilled water and standardized against potassium dichromate after at least 2 weeks storage.

0.01N: Dilute 10 times with freshly boiled distilled water, standardized against potassium dichromate.

0.001N: Dilute 100 times with freshly boiled distilled water, standardized against potassium dichromate.

Sodium thiosulfate solution must be calibrated by standard $\mathrm{K}_{2} \mathrm{Cr}_{2} \mathrm{O}_{7}$ solution. 


\section{Appendix III}

\section{Calibration of Sodium Thiosulfate solution}

In $80.0 \mathrm{ml}$ distilled water slowly add in $1.0 \mathrm{ml}$ smoking $\mathrm{H}_{2} \mathrm{SO}_{4}$ and stirred, then added in $1.0 \mathrm{~g} \mathrm{KI} \cdot 5 \mathrm{H}_{2} \mathrm{O}$, precisely measured $10.00 \mathrm{ml} 0.1000 \mathrm{~N} \mathrm{~K}_{2} \mathrm{Cr}_{2} \mathrm{O}_{7}$ solution add in the solution, shook and kept in dark for reaction of 6 minutes. Use sodium thiosulfate solution to titrate, record the consumption [APHA. 1992].

Standard 0.1000N $\mathrm{K}_{2} \mathrm{Cr}_{2} \mathrm{O}_{7}$ making: dissolve $4.904 \mathrm{~g}$ anhydrous potassium dichromate of primary standard quality in distilled water and diluted to $1000 \mathrm{ml}$ to yield a $0.1000 \mathrm{~N}$ solution. Then stored in a glass-stopper bottle.

Calculation is:

Normality of sodium thiosulfate $=0.1 \times 10 \mathrm{ml}$ of $\mathrm{K}_{2} \mathrm{Cr}_{2} \mathrm{O}_{7} /$ consumption of sodium thiosulfate solution 


\section{Appendix IV}

\section{Calculation of permeate flux}

Automatic weighted and recorded the accumulated gram each 5 second by the Ohaus AV2101 auto balance.

Time (5s): $\quad t_{1}, \quad t_{2}, \quad t_{3}, \ldots \ldots, t_{n}$

Weight $(\mathrm{g}): \quad \mathrm{g}_{1}, \quad \mathrm{~g}_{2}, \quad \mathrm{~g}_{3}, \ldots \ldots, \mathrm{g}_{\mathrm{n}}$

Flowrate $(\mathrm{g} / \mathrm{s}) \quad \mathrm{g}_{1} / \mathrm{t}_{1},\left(\mathrm{~g}_{2}-\mathrm{g}_{1}\right) /\left(\mathrm{t}_{2}-\mathrm{t}_{1}\right), \ldots . .,\left(\mathrm{g}_{\mathrm{n}}-\mathrm{g}_{\mathrm{n}-1}\right) /\left(\mathrm{t}_{\mathrm{n}}-\mathrm{t}_{\mathrm{n}-1}\right)$ 


\section{Appendix V}

\section{Test and Calculation of Ozone Concentration}

\section{REAGENTS}

Indigo stock solution: Dissolve $192.5 \mathrm{mg}$ of potassium indigo trisulfonate in deionized water and dilute to volume in a $250 \mathrm{~mL}$ volumetric flask. The stock solution is stable for about 4 months when stored in the dark.

Indigo reagent II: Prepare by adding $25 \mathrm{~mL}$ of the Indigo stock, $2.5 \mathrm{~g}$ of sodium dihydrogen phosphate and $1.75 \mathrm{~mL}$ of concentrated phosphoric acid to a $250 \mathrm{~mL}$ volumetric flask and diluting to volume with deionized water. The solution should be fine to use within a week.

\section{TEST}

Pipet $3.0 \mathrm{~mL}$ of Indigo Reagent II into a $10 \mathrm{~mL}$ volumetric flask and dilute to $10 \mathrm{~mL}$. Repeat so that a volumetric flask is prepared for each sample and the blank. Pipet $1.0 \mathrm{ml}$ ozone solution into the flask and shake immediately.

Determine the absorbance of the blank and samples at $600 \mathrm{~nm}$ using a one cm cuvette. Absorbance values should be determined as soon as possible.

\section{CALCULATION}

Determine the concentration of ozone in the sample using the following equation:

$\mathrm{mg} / \mathrm{L} \mathrm{O} \mathrm{O}_{3}=\frac{\text { Final.sample.vol. }(\mathrm{ml}) \times \Delta A}{f \times b \times V}$

where: final sample vol. $(\mathrm{mL})=$ volume sample is diluted to in volumetric flask

$\Delta A=$ difference in absorbance between blank and sample (blank $\mathrm{A}-$ sample $\mathrm{A}$ )

$f=0.42($ constant $)$

$b=$ path length of absorbance cell, $\mathrm{cm}$

$V=$ volume of sample, $\mathrm{mL}$

(Markee et al., 2009) 


\section{Appendix VI}

\section{A summary of the principle infrared bands and their assignments.}

$\mathrm{R}$ is an aliphatic group.

[Infrared Spectroscopy pp11 of 26, http://www.umsl.edu/ orglab/documents/IR/IR2.html]

\begin{tabular}{|c|c|c|c|c|}
\hline $\begin{array}{l}\text { Functional } \\
\text { Group }\end{array}$ & Type & & $\begin{array}{l}\text { Frequencies } \\
\mathrm{cm}-1\end{array}$ & $\begin{array}{l}\text { Peak } \\
\text { Intensity }\end{array}$ \\
\hline \multirow[t]{4}{*}{$\mathrm{C}-\mathrm{H}$} & sp3 hybridized & R3C-H & $2850-3000$ & M (sh) \\
\hline & sp2 hybridized & $=\mathrm{CR}-\mathrm{H}$ & $3000-3250$ & M(sh) \\
\hline & sp hybridized & $\mathrm{C}-\mathrm{H}$ & 3300 & M-S (sh) \\
\hline & aldehyde $\mathrm{C}-\mathrm{H}$ & $\mathrm{H}-(\mathrm{C}=0) \mathrm{R}$ & $2750, \quad 2850$ & M(sh) \\
\hline \multirow[t]{3}{*}{$\mathrm{N}-\mathrm{H}$} & $\begin{array}{l}\text { primary amine, } \\
\text { amide }\end{array}$ & $\mathrm{RN}-\mathrm{H} 2, \quad \mathrm{RCON}-\mathrm{H} 2$ & 3300,3340 & S, S (br) \\
\hline & $\begin{array}{l}\text { secondary amine, } \\
\text { amide }\end{array}$ & RNR-H, RCON-HR & $3300-3500$ & $S(b r)$ \\
\hline & $\begin{array}{ll}\text { tertiary amine, } \\
\text { amide }\end{array}$ & RN (R3), RCONR2 & none & \\
\hline \multirow[t]{3}{*}{ O-H } & alcohols, phenols & free $0-\mathrm{H}$ & $3620-3580$ & W(sh) \\
\hline & & hydrogen bonded & $3600-3650$ & $S(b r)$ \\
\hline & carboxylic acids & $\mathrm{R}(\mathrm{C}=0) 0-\mathrm{H}$ & $3500-2400$ & $S$ (br) \\
\hline $\mathrm{CN}$ & nitriles & $\mathrm{RCN}$ & $2280-2200$ & S(sh) \\
\hline \multirow[t]{2}{*}{$\mathrm{CC}$} & acetylenes & $\mathrm{R}-\mathrm{CC}-\mathrm{R}$ & $2260-2180$ & W(sh) \\
\hline & & $\mathrm{R}-\mathrm{CC}-\mathrm{H}$ & $2160-2100$ & $\mathrm{M}(\mathrm{sh})$ \\
\hline \multirow[t]{5}{*}{$C=0$} & aldehydes & $\mathrm{R}(\mathrm{C}=0) \mathrm{H}$ & $1740-1720$ & $\mathrm{~S}(\mathrm{sh})$ \\
\hline & ketones & $\mathrm{R}(\mathrm{C}=0) \mathrm{R}$ & \begin{tabular}{|l}
$1730-1710$ \\
\end{tabular} & S(sh) \\
\hline & esters & $\mathrm{R}(\mathrm{C} 02) \mathrm{R}$ & \begin{tabular}{|l|l|}
$1750-1735$ \\
\end{tabular} & S(sh) \\
\hline & anhydrides & $\mathrm{R}(\mathrm{CO} 2 \mathrm{CO}) \mathrm{R}$ & $1820, \quad 1750$ & S, S(sh) \\
\hline & carboxylates & $\mathrm{R}(\mathrm{CO} 2) \mathrm{H}$ & 1600,1400 & $\mathrm{~S}, \mathrm{~S}(\mathrm{sh})$ \\
\hline \multirow[t]{3}{*}{$\mathrm{C}=\mathrm{C}$} & olefins & $\mathrm{R} 2 \mathrm{C}=\mathrm{CR} 2$ & 1680-1640 & W(sh) \\
\hline & & $\mathrm{R} 2 \mathrm{C}=\mathrm{CH} 2$ & $1600-1675$ & $M(\mathrm{sh})$ \\
\hline & & R2C $=C$ (OR) R & $1600-1630$ & S(sh) \\
\hline$-\mathrm{N} 02$ & nitro groups & RN02 & 1550,1370 & $\mathrm{~S}, \mathrm{~S}(\mathrm{sh})$ \\
\hline
\end{tabular}

Intensities as follows: $\mathrm{w}=$ weak, $\mathrm{m}=$ =medium, $\mathrm{s}=\mathrm{strong}, \mathrm{sh}=$ shoulder. 


\section{Appendix VII}

\section{Experimental Data Tables of PPMM reaction with ozone}

\section{Table VII-1 Peroxide data table of PPMM in gaseous phase}

The PPMM sample pieces $(90 \mathrm{~mm} \times 0.45 \mu)$ were fixed in reactor and treated by gaseous ozone. Ozone flow was in $10 \mathrm{~L} / \mathrm{min}$ at concentration of $3.0 \mathrm{wt} \%$ and pressure of $15 \mathrm{psig}$.

Ozonated samples were degassed by vacuum in more than $1.5 \mathrm{hrs}$.

The standard iodometric titration was applied to test the peroxide concentration.

\begin{tabular}{|c|c|c|c|c|c|c|c|c|c|c|}
\hline \multirow{2}{*}{$\begin{array}{l}\text { Ozone } \\
\text { \%wt }\end{array}$} & \multirow{2}{*}{$\begin{array}{l}\text { Time } \\
\text { hr }\end{array}$} & \multicolumn{3}{|c|}{$\begin{array}{l}0.00084 \mathrm{~N} \mathrm{Na}_{2} \mathrm{~S}_{2} \mathrm{O}_{3} \text { Titration } \\
\text { consumption } \mathrm{ml}\end{array}$} & \multicolumn{3}{|c|}{$\begin{array}{l}\text { Peroxide Concentration } \\
\qquad \mathrm{mmol} / \mathrm{m}^{2}\end{array}$} & \multirow{2}{*}{$\begin{array}{l}\text { Avera } \\
\text { ge } \\
\text { mmol/ } \\
\mathrm{m}^{2}\end{array}$} & \multirow{2}{*}{\multicolumn{2}{|c|}{ Error }} \\
\hline & & $\begin{array}{c}\text { Sample } \\
1\end{array}$ & $\begin{array}{c}\text { Sample } \\
2\end{array}$ & $\begin{array}{l}\text { Sample } \\
3\end{array}$ & $\begin{array}{c}\text { Sample } \\
1 \\
\end{array}$ & $\begin{array}{c}\text { Sample } \\
2 \\
\end{array}$ & $\begin{array}{l}\text { Samp1 } \\
\text { e } 3 \\
\end{array}$ & & & \\
\hline 3.0 & 0.25 & 13 & 12.9 & 12.8 & 0.4293 & 0.4260 & 0.4194 & 0.4211 & -0.0116 & 0.0083 \\
\hline 3.0 & 0.5 & 21 & 21.5 & 20.2 & 0.6936 & 0.7111 & 0.6853 & 0.6967 & -0.0114 & 0.0144 \\
\hline 3.0 & 1.0 & 34.2 & 33.6 & 35.0 & 1.1295 & 1.1101 & 1.1354 & 1.1250 & -0.0149 & 0.0104 \\
\hline 3.0 & 1.25 & 42.4 & & & 1. 4001 & & & 1.4001 & & \\
\hline 3.0 & 1.50 & 50.2 & 51.5 & 49.8 & 1.6579 & 1.7015 & 1.6873 & 1.6822 & -0.0243 & 0.0193 \\
\hline 3.0 & 1.75 & 58.3 & 57.5 & 59.8 & 1.9255 & 1.8985 & 1.9458 & 1.9233 & -0.0248 & 0.0225 \\
\hline$A V G$ & & & & & & & & & -0.0174 & 0.0150 \\
\hline
\end{tabular}




\section{Appendix VII}

\section{Experimental Data Tables of PPMM reaction with ozone}

\section{Table VII-2 Peroxide data table of PPMM in aqueous phase}

The PPMM sample pieces $(90 \mathrm{~mm} \times 0.45 \mu)$ were fixed in reactor and treated by aqueous ozonation.

The reactor was filled with $10 \mathrm{~L}$ distilled water.

Ozone flow was in $10 \mathrm{~L} / \mathrm{min}$ at concentration of $3.0 \mathrm{wt} \%$ and pressure of $15 \mathrm{psig}$, ozone bubbled in water.

Ozonated samples were dried and degassed by vacuum in more than $1.5 \mathrm{hrs}$.

The standard iodometric titration was applied to test the peroxide concentration.

\begin{tabular}{|c|c|c|c|c|c|c|c|c|c|c|}
\hline \multirow{2}{*}{$\begin{array}{l}\text { Ozone } \\
\text { \%wt }\end{array}$} & \multirow{2}{*}{$\begin{array}{c}\text { Time } \\
\text { hr }\end{array}$} & \multicolumn{3}{|c|}{$\begin{array}{c}\text { 0. } 00084 \mathrm{~N} \mathrm{Na}_{2} \mathrm{~S}_{2} \mathrm{O}_{3} \text { Titration } \\
\text { consumption } \mathrm{ml}\end{array}$} & \multicolumn{3}{|c|}{$\begin{array}{c}\text { Peroxide Concentration } \\
\mathrm{mmol} / \mathrm{m}^{2}\end{array}$} & \multirow{2}{*}{$\begin{array}{l}\text { Average } \\
\mathrm{mmol} / \mathrm{m}^{2}\end{array}$} & \multirow{2}{*}{\multicolumn{2}{|c|}{ Error }} \\
\hline & & $\begin{array}{c}\text { Sample } \\
1\end{array}$ & $\begin{array}{c}\text { Sample } \\
2\end{array}$ & $\begin{array}{c}\text { Sample } \\
3\end{array}$ & $\begin{array}{c}\text { Sample } \\
1\end{array}$ & $\begin{array}{c}\text { Sample } \\
2\end{array}$ & $\begin{array}{c}\text { Sample } \\
3\end{array}$ & & & \\
\hline 3.0 & 0.25 & 12.2 & 12.1 & 12.8 & 0.4029 & 0.3987 & 0.424 & 0.4085 & -0.0098 & 0.0155 \\
\hline 3.0 & 0.5 & 18.9 & 19.3 & 18.2 & 0.6242 & 0.6385 & 0.6023 & 0.6217 & -0.0194 & 0.0168 \\
\hline 3.0 & 1 & 30.8 & 32.2 & 30.4 & 1.0172 & 1.0636 & 1.0032 & 1.0280 & -0.0248 & 0.0356 \\
\hline 3.0 & 1.25 & 38.1 & 37.1 & 37.6 & 1.2583 & 1.2257 & 1.243 & 1.2423 & -0.0166 & 0.0007 \\
\hline 3.0 & 1.5 & 43.2 & 43.5 & 42.6 & 1.4280 & 1.438 & 1.4076 & 1.4245 & -0.0169 & 0.0135 \\
\hline 3.0 & 1.75 & 47.6 & 49.6 & 48.1 & 1.5721 & 1.6376 & 1.5891 & 1. 59959 & -0.0275 & 0.0380 \\
\hline AVG & & & & & & & & & -0.0192 & 0.0200 \\
\hline
\end{tabular}




\section{Appendix VIII}

\section{Experimental Data Tables of scavenger test of PPMM reaction with ozone}

Table VIII Peroxide data table of PPMM in aqueous medium with scavenger- Sodium Carbonate Concentration $3 \mathrm{~g} / \mathrm{L}$

The PPMM sample pieces were fixed in reactor and treated by aqueous ozonation.

The reactor was filled with $10 \mathrm{~L}$ distilled water and with scavenger- Sodium Carbonate dissolved in.

Ozone flow was in $10 \mathrm{~L} / \mathrm{min}$ at concentration of $3.0 \mathrm{wt} \%$ and pressure of $15 \mathrm{psig}$, ozone bubbled in water.

Ozonated samples were dried and degassed by vacuum in more than $1.5 \mathrm{hrs}$.

The standard iodometric titration was applied to test the peroxide concentration.

\begin{tabular}{|c|c|c|c|c|c|c|c|c|c|}
\hline \multirow{2}{*}{$\begin{array}{l}\text { Ozone } \\
\text { \%wt }\end{array}$} & \multirow{2}{*}{$\begin{array}{l}\text { Time } \\
\text { hr }\end{array}$} & \multicolumn{3}{|c|}{$\begin{array}{l}0.00084 \mathrm{~N} \mathrm{Na}_{2} \mathrm{~S}_{2} \mathrm{O}_{3} \text { Titration } \\
\text { consumption } \mathrm{ml}\end{array}$} & \multicolumn{3}{|c|}{$\begin{array}{l}\text { Peroxide Concentration } \\
\mathrm{mmol} / \mathrm{m}^{2}\end{array}$} & \multirow{2}{*}{$\begin{array}{l}\text { Average } \\
\mathrm{mmol} / \mathrm{m}^{2}\end{array}$} & \multirow{2}{*}{$\begin{array}{l}\text { Error } \\
+/-\end{array}$} \\
\hline & & Sample 1 & $\begin{array}{c}\text { Sample } \\
2\end{array}$ & $\begin{array}{c}\text { Sample } \\
3\end{array}$ & $\begin{array}{c}\text { Sample } \\
1\end{array}$ & $\begin{array}{c}\text { Sample } \\
2\end{array}$ & $\begin{array}{c}\text { Sample } \\
3\end{array}$ & & \\
\hline 3.0 & 0.25 & 10.7 & 10.9 & & 0.3534 & 0.3600 & & 0.3567 & 0.0033 \\
\hline 3.0 & 0.5 & 17.5 & 18.1 & & 0.5780 & 0.5978 & & 0.5879 & 0.0099 \\
\hline 3.0 & 1.25 & 26.3 & 25.6 & & 0.8686 & 0.8455 & & 0.8570 & 0.0231 \\
\hline 3.0 & 1.5 & 29.4 & 29 & & 0.9710 & 0.9578 & & 0.9644 & 0.0132 \\
\hline 3.0 & 1.75 & 32.3 & 32.8 & & 1.0668 & 1.0833 & & 1.0750 & 0.0083 \\
\hline $\mathrm{AVG}$ & & & & & & & & & 0.0116 \\
\hline
\end{tabular}




\section{Appendix IX}

\section{Experimental Data Tables of washing test of PPMM reaction with ozone}

Table IX-1 Peroxide data table of PPMM in gaseous medium with/without $10 \%$ IPA + distilled water washing 15 minutes

The PPMM sample pieces $(90 \mathrm{~mm} \times 0.45 \mu)$ were fixed in reactor and treated by gaseous ozone. Ozone flow was in $10 \mathrm{~L} / \mathrm{min}$ at concentration of $3.0 \mathrm{wt} \%$ and pressure of $15 \mathrm{psig}$.

Ozonated samples were split in half, one half was washed by $10 \%$ IPA + distilled water, and another half was not washed. Then dried and degassed by vacuum in more than $1.5 \mathrm{hrs}$.

The standard iodometric titration was applied to test the peroxide concentration.

\begin{tabular}{|c|c|c|c|c|c|c|c|c|}
\hline \multirow{2}{*}{$\begin{array}{l}\text { Ozone } \\
\% w t\end{array}$} & \multirow{2}{*}{$\begin{array}{l}\text { Time } \\
\text { hr }\end{array}$} & \multicolumn{2}{|c|}{$\begin{array}{c}0.00084 \mathrm{~N} \mathrm{Na}_{2} \mathrm{~S}_{2} \mathrm{O}_{3} \\
\text { Titration consumption } \\
\text { ml-Non-washing }\end{array}$} & \multicolumn{3}{|c|}{$\begin{array}{l}\text { Peroxide Concentration } \\
\mathrm{mmol} / \mathrm{m}^{2} \text {-Non-washing }\end{array}$} & \multirow{2}{*}{$\begin{array}{l}\text { Average } \\
\mathrm{mmol} / \mathrm{m}^{2}\end{array}$} & \multirow{2}{*}{$\begin{array}{l}\text { Error } \\
+/-\end{array}$} \\
\hline & & Sample 1 & $\begin{array}{l}\text { Sample } \\
2\end{array}$ & \multicolumn{2}{|c|}{ Sample 1} & $\begin{array}{l}\text { Sample } \\
2\end{array}$ & & \\
\hline 3.0 & 0.25 & 12.1 & 12.7 & \multicolumn{2}{|c|}{0.3996} & 0.4194 & 0.4095 & 0.0099 \\
\hline 3.0 & 0.5 & 19.4 & 19.8 & \multicolumn{2}{|c|}{0.6407} & 0.6539 & 0.6473 & -0.0066 \\
\hline 3.0 & 0.75 & 25.7 & 26.6 & \multicolumn{2}{|c|}{0.8488} & 0.8785 & 0.8636 & 0.0149 \\
\hline $\mathrm{AVG}$ & & & & & & & & 0.0061 \\
\hline \multirow{2}{*}{$\begin{array}{l}\text { Ozone } \\
\text { \%wt }\end{array}$} & \multirow{2}{*}{$\begin{array}{l}\text { Time } \\
\text { hr }\end{array}$} & \multicolumn{2}{|c|}{$\begin{array}{c}0.00084 \mathrm{~N} \mathrm{Na}_{2} \mathrm{~S}_{2} \mathrm{O}_{3} \\
\text { Titration consumption } \\
\text { ml-washed }\end{array}$} & \multicolumn{2}{|c|}{$\begin{array}{c}\text { Peroxide } \\
\text { Concentration } \\
\mathrm{mmol} / \mathrm{m}^{2} \text {-washed }\end{array}$} & \multirow{2}{*}{\multicolumn{2}{|c|}{ Average $\mathrm{mmol} / \mathrm{m}^{2}$}} & \multirow[t]{2}{*}{$\begin{array}{l}\text { Error } \\
+/-\end{array}$} \\
\hline & & Sample 1 & $\begin{array}{l}\text { Sample } \\
2 \\
\end{array}$ & $\begin{array}{c}\text { Sample } \\
1 \\
\end{array}$ & Sample 2 & & & \\
\hline 3.0 & 0.25 & 10.8 & 10.5 & 0.3567 & 0.3468 & \multicolumn{2}{|c|}{0.3517} & 0.0050 \\
\hline 3.0 & 0.5 & 15.9 & 15.3 & 0.5251 & 0.5053 & \multicolumn{2}{|c|}{0.5152} & 0.0099 \\
\hline 3.0 & 0.75 & 22.8 & 22.0 & 0.7530 & 0.7266 & \multirow{2}{*}{\multicolumn{2}{|c|}{0.7398}} & 0.0132 \\
\hline$A V G$ & & & & & & & & 0.0094 \\
\hline
\end{tabular}




\section{Appendix IX}

\section{Experimental Data Tables of washing test of PPMM reaction with ozone}

Table IX-2 Peroxide data table of PPMM in aqueous medium with/without $10 \%$ IPA + distilled water washing 15 minutes

The PPMM sample pieces were fixed in reactor and treated by aqueous ozonation. Ozone flow was in $10 \mathrm{~L} / \mathrm{min}$ at concentration of $3.0 \mathrm{wt} \%$ and pressure of $15 \mathrm{psig}$, ozone bubbled in water.

Ozonated samples were split in half, one half was washed by $10 \%$ IPA + distilled water, and another half was not washed. Then dried and degassed by vacuum in more than $1.5 \mathrm{hrs}$.

The standard iodometric titration was applied to test the peroxide concentration.

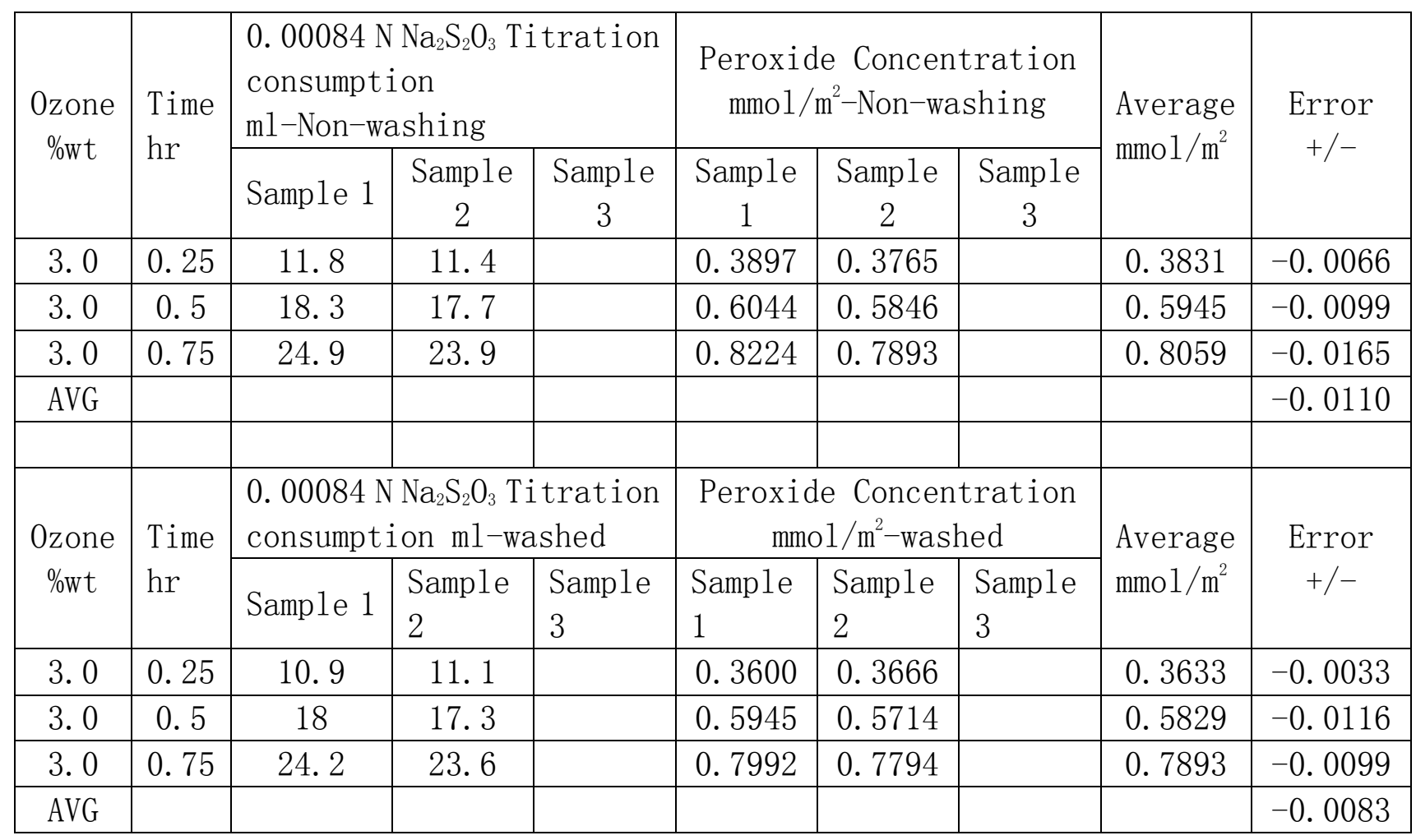




\section{Appendix X}

\section{Experimental Data Tables of Pre-wet test of PPMM reaction with ozone}

Table X Peroxide data table of PPMM in aqueous medium with/without 1 minute IPA pre-wet +15 minutes distilled water washing

The PPMM sample pieces were split in half, one half was soaked in IPA liquid as pre-wet in one minute then washed the IPA away by distilled water in 15 minutes, another half was used directly. Then fixed the samples in reactor and treated by aqueous ozonation.

Ozone flow was in $10 \mathrm{~L} / \mathrm{min}$ at concentration of $3.0 \mathrm{wt} \%$ and pressure of $15 \mathrm{psig}$, ozone bubbled in water.

The standard iodometric titration was applied to test the peroxide concentration.

\begin{tabular}{|c|c|c|c|c|c|c|c|c|c|}
\hline \multirow{3}{*}{$\begin{array}{l}\text { Ozone } \\
\text { \%wt }\end{array}$} & \multirow{3}{*}{$\begin{array}{l}\text { Time } \\
\text { hr }\end{array}$} & \multicolumn{3}{|c|}{$\begin{array}{l}0.00084 \mathrm{~N} \mathrm{Na}_{2} \mathrm{~S}_{2} \mathrm{O}_{3} \text { Titration } \\
\text { consumption } \mathrm{ml}\end{array}$} & \multicolumn{3}{|c|}{$\begin{array}{l}\text { Peroxide Concentration } \\
\mathrm{mmol} / \mathrm{m}^{2}\end{array}$} & \multirow{3}{*}{$\begin{array}{l}\text { Pre-wet } \\
\text { Average } \\
\mathrm{mmol} / \mathrm{m}^{2}\end{array}$} & \multirow{3}{*}{$\begin{array}{c}\text { Pre-wet } \\
\text { Error } \\
+/-\end{array}$} \\
\hline & & \multicolumn{2}{|l|}{ Pre-wet } & \multirow{2}{*}{\begin{tabular}{|l}
$\begin{array}{l}\text { Not } \\
\text { wet }\end{array}$ \\
Sample \\
1 \\
\end{tabular}} & \multicolumn{2}{|c|}{ Pre-wet } & \multirow{2}{*}{$\begin{array}{c}\text { Not } \\
\text { wet } \\
\text { Sample } \\
1\end{array}$} & & \\
\hline & & Sample 1 & $\begin{array}{c}\text { Sample } \\
2\end{array}$ & & $\begin{array}{c}\text { Sample } \\
1\end{array}$ & $\begin{array}{c}\text { Sample } \\
2 \\
\end{array}$ & & & \\
\hline 3.0 & 0.25 & 12.85 & 13.1 & 12.2 & 0.4244 & 0.4326 & 0.4029 & 0.4285 & 0.0041 \\
\hline 3.0 & 0.5 & 20.8 & 20.9 & 18.9 & 0.6870 & 0.6903 & 0.6242 & 0.6886 & 0.0017 \\
\hline 3.0 & 1.0 & 28.3 & 27.9 & 30.8 & 0.9347 & 0.9214 & 1.0172 & 0.9280 & 0.0066 \\
\hline 3.0 & 1.25 & 31.2 & 32.2 & 38.1 & 1.0304 & 1.0635 & 1.2583 & 1.0469 & 0.0165 \\
\hline $\mathrm{AVG}$ & & & & & & & & & 0.0072 \\
\hline
\end{tabular}




\section{Appendix XI}

\section{Experimental Data Tables of different $\mathrm{pH}$ test of PPMM reaction with}

\section{ozone}

Table XI-1 Peroxide data table of PPMM in aqueous medium at pH 2.3

The PPMM sample pieces were fixed in reactor and treated by aqueous ozonation.

The reactor was filled with $10 \mathrm{~L}$ distilled water and set $\mathrm{pH}$ at 2.3 adjusted by $1.0 \mathrm{M} \mathrm{H}_{2} \mathrm{SO}_{4}$ and $\mathrm{NaOH}$.

Ozone flow was in $10 \mathrm{~L} / \mathrm{min}$ at concentration of $3.0 \mathrm{wt} \%$ and pressure of $15 \mathrm{psig}$, ozone bubbled in water.

Ozonated samples were dried and degassed by vacuum in more than $1.5 \mathrm{hrs}$.

The standard iodometric titration was applied to test the peroxide concentration.

\begin{tabular}{|c|c|c|c|c|c|}
\hline $\begin{array}{l}\text { Ozone \%wt } \\
\text { non-washing }\end{array}$ & Time hr & $\begin{array}{l}\text { 0.000909 } \mathrm{N} \mathrm{Na}_{2} \mathrm{~S}_{2} \mathrm{O}_{3} \\
\text { Titration } \\
\text { consumption } \mathrm{ml}\end{array}$ & $\begin{array}{l}\text { Molality of } \\
\text { peroxide } \\
\text { mmol }\end{array}$ & $\begin{array}{l}\text { Area of } \\
\text { samples } \mathrm{m}^{2}\end{array}$ & mmol/m \\
\hline 3.0 & $0.25 \mathrm{hr}$ & 3.8 & 0.0017 & 0.003468 & 0.4936 \\
\hline 3.0 & $0.5 \mathrm{hr}$ & 5.4 & 0.0028 & 0.003468 & 0.7014 \\
\hline 3.0 & $0.75 \mathrm{hr}$ & 8.1 & 0.0042 & 0.003468 & 1.0520 \\
\hline 3.0 & $1.25 \mathrm{HR}$ & 10.4 & 0.0049 & 0.003468 & 1.3508 \\
\hline
\end{tabular}




\section{Appendix XI}

\section{Experimental Data Tables of different pH test of PPMM reaction with}

\section{ozone}

Table XI-2 Peroxide data table of PPMM in aqueous medium at pH 5.35 -distilled water

The PPMM sample pieces were fixed in reactor and treated by aqueous ozonation.

The reactor was filled with $10 \mathrm{~L}$ distilled water and $\mathrm{pH}$ was tested to be 5.35.

Ozone flow was in $10 \mathrm{~L} / \mathrm{min}$ at concentration of $3.0 \mathrm{wt} \%$ and pressure of $15 \mathrm{psig}$, ozone bubbled in water.

Ozonated samples were dried and degassed by vacuum in more than $1.5 \mathrm{hrs}$.

The standard iodometric titration was applied to test the peroxide concentration.

\begin{tabular}{|c|c|c|c|c|c|}
\hline $\begin{array}{l}\text { Ozone \%wt } \\
\text { non-washing }\end{array}$ & Time hr & $\begin{array}{c}\text { 0.000909 } \\
\text { Titration } \\
\text { consumption } \mathrm{ml}\end{array}$ & $\begin{array}{c}\text { Molality } \\
\text { of } \\
\text { peroxide } \\
\text { mmol }\end{array}$ & $\begin{array}{c}\text { Area of } \\
\text { samples } \\
\mathrm{m}^{2}\end{array}$ & $\mathrm{mmol} / \mathrm{m}^{2}$ \\
\hline 3.0 & $0.25 \mathrm{hr}$ & 3.5 & 0.001505 & 0.003468 & 0.4340 \\
\hline 3.0 & $0.5 \mathrm{hr}$ & 5.6 & 0.002408 & 0.003468 & 0.6943 \\
\hline 3.0 & $0.75 \mathrm{hr}$ & 8 & 0.00344 & 0.003468 & 0.9919 \\
\hline 3.0 & $1.25 \mathrm{HR}$ & 10.9 & 0.004687 & 0.003468 & 1.3514 \\
\hline
\end{tabular}




\section{Appendix XI}

\section{Experimental Data Tables of different $\mathrm{pH}$ test of PPMM reaction with}

\section{ozone}

Table XI-3 Peroxide data table of PPMM in aqueous medium at pH 10.0

The PPMM sample pieces were fixed in reactor and treated by aqueous ozonation.

The reactor was filled with $10 \mathrm{~L}$ distilled water and set $\mathrm{pH}$ at 10.0 adjusted by $1.0 \mathrm{M} \mathrm{H}_{2} \mathrm{SO}_{4}$ and $\mathrm{NaOH}$.

Ozone flow was in $10 \mathrm{~L} / \mathrm{min}$ at concentration of $3.0 \mathrm{wt} \%$ and pressure of $15 \mathrm{psig}$, ozone bubbled in water.

Ozonated samples were dried and degassed by vacuum in more than $1.5 \mathrm{hrs}$.

The standard iodometric titration was applied to test the peroxide concentration.

\begin{tabular}{|c|c|c|c|c|c|}
\hline $\begin{array}{l}\text { Ozone \%wt } \\
\text { non-washing }\end{array}$ & Time hr & $\begin{array}{c}0.000909 \mathrm{~N} \mathrm{Na}_{2} \mathrm{~S}_{2} \mathrm{O}_{3} \\
\text { Titration } \\
\text { consumption } \mathrm{ml}\end{array}$ & $\begin{array}{c}\text { Molality of } \\
\text { peroxide } \\
\text { mmol }\end{array}$ & $\begin{array}{c}\text { Area of } \\
\text { samples } \mathrm{m}^{2}\end{array}$ & $\mathrm{mmol} / \mathrm{m}^{2}$ \\
\hline 3.0 & 0.25 & 3.2 & 0.001376 & 0.003468 & 0.3968 \\
\hline 3.0 & 0.5 & 4.9 & 0.002107 & 0.003468 & 0.6075 \\
\hline 3.0 & 0.75 & 7 & 0.00301 & 0.003468 & 0.8679 \\
\hline 3.0 & 1.25 & 7.8 & 0.003354 & 0.003468 & 0.9671 \\
\hline
\end{tabular}




\section{Appendix XI}

\section{Experimental Data Tables of different $\mathrm{pH}$ test of PPMM reaction with}

\section{ozone}

\section{Table XI-4 Peroxide data table of PPMM in aqueous medium at different pH}

The PPMM sample pieces were fixed in reactor and treated by aqueous ozonation.

The reactor was filled with $10 \mathrm{~L}$ distilled water and set different $\mathrm{pH}$, respectively, adjusted by $1.0 \mathrm{M} \mathrm{H}_{2} \mathrm{SO}_{4}$ and $\mathrm{NaOH}$.

Ozone flow was in $10 \mathrm{~L} / \mathrm{min}$ at concentration of $3.0 \mathrm{wt} \%$ and pressure of $15 \mathrm{psig}$, ozone bubbled in water. Ozonation time is $30 \mathrm{~min}$.

Ozonated samples were dried and degassed by vacuum in more than $1.5 \mathrm{hrs}$.

The standard iodometric titration was applied to test the peroxide concentration.

\begin{tabular}{|c|c|c|c|c|c|c|c|c|c|}
\hline $\begin{array}{l}\text { Reaction } \\
\text { time }\end{array}$ & Ozone & $\mathrm{pH}=2.3$ & & $\mathrm{pH}=4.0$ & & $\mathrm{pH}=5.0$ & & $\mathrm{pH}=6.0$ & \\
\hline \multirow{3}{*}{ minutes } & & $9.0 \times 10^{-4}$ & Peroxide & $8.4 \times 10^{-4}$ & Peroxide & $8.4 \times 10^{-4}$ & Peroxide & & Peroxide \\
\hline & $3.0 \mathrm{wt} \%$ & $\mathrm{~N}$ & CONC & $\mathrm{N}$ & CONC & $\mathrm{N}$ & CONC & $8.4 \times 10^{-4} \mathrm{~N}$ & CONC \\
\hline & & $\mathrm{ml}$ & $\mathrm{mmol} / \mathrm{M}^{2}$ & $\mathrm{ml}$ & $\mathrm{mmol} / \mathrm{M}^{2}$ & $\mathrm{ml}$ & $\mathrm{mmol} / \mathrm{M}^{2}$ & $\mathrm{ml}$ & $\mathrm{mmol} / \mathrm{M}^{2}$ \\
\hline 30 & Sample 1 & 5.4 & 0.7014 & 7 & 0.8477 & 5.9 & 0.7145 & 5.5 & 0.6661 \\
\hline \multirow[t]{2}{*}{30} & Sample 2 & & & 6.2 & 0.7508 & 5.8 & 0.7024 & 6.1 & 0.7387 \\
\hline & AVG & & 0.7014 & & 0.7993 & & 0.7085 & & 0.7024 \\
\hline $\begin{array}{c}\text { Reaction } \\
\text { time }\end{array}$ & Ozone & $\mathrm{pH}=7.0$ & & $\mathrm{pH}=8.0$ & & $\mathrm{pH}=9.0$ & & $\mathrm{pH}=10.0$ & \\
\hline \multirow{3}{*}{ minutes } & & $9.0 \times 10^{-4}$ & Peroxide & $8.4 \times 10-4$ & Peroxide & $8.4 \times 10-4$ & Peroxide & & Peroxide \\
\hline & $3.0 \mathrm{wt} \%$ & $\mathrm{~N}$ & CONC & $\mathrm{N}$ & CONC & $\mathrm{N}$ & CONC & $8.4 \times 10-4 \mathrm{~N}$ & CONC \\
\hline & & $\mathrm{ml}$ & $\mathrm{mmol} / \mathrm{M}^{2}$ & $\mathrm{ml}$ & $\mathrm{mmol} / \mathrm{M} 2$ & $\mathrm{ml}$ & $\mathrm{mmol} / \mathrm{M} 2$ & $\mathrm{ml}$ & $\mathrm{mmol} / \mathrm{M} 2$ \\
\hline 30 & Sample 1 & 6.4 & 0.7935 & 4.15 & 0.5026 & 4.5 & 0.5450 & 4.9 & 0.6075 \\
\hline \multirow[t]{2}{*}{30} & Sample 2 & & & 4.2 & 0.5086 & 4.55 & 0.5510 & & \\
\hline & $\mathrm{AVG}$ & & 0.7935 & & 0.5056 & & 0.5480 & & 0.6075 \\
\hline
\end{tabular}




\section{Appendix XII}

\section{Experimental Data Tables of catalyst test of PPMM reaction with ozone}

Table XII-1 Peroxide data table of PPMM in aqueous medium at $0.1 \mathrm{~g} / \mathrm{L} \mathrm{Cu}^{2+}$ catalyst

The PPMM sample pieces were fixed in reactor and treated by aqueous ozonation.

The reactor was filled with $10 \mathrm{~L}$ distilled water and $0.1 \mathrm{~g} / \mathrm{L}(3.148 \mathrm{mmol} / \mathrm{L}) \mathrm{Cu}^{2+}$ catalyst was added (with $\mathrm{CuSO}_{4} \cdot 5 \mathrm{H}_{2} \mathrm{O}$ ).

Ozone flow was in $10 \mathrm{~L} / \mathrm{min}$ at concentration of $3.0 \mathrm{wt} \%$ and pressure of $15 \mathrm{psig}$, ozone bubbled in water.

Ozonated samples were dried and degassed by vacuum in more than $1.5 \mathrm{hrs}$.

The standard iodometric titration was applied to test the peroxide concentration.

\begin{tabular}{|c|c|c|c|c|c|}
\hline $\begin{array}{l}\text { Ozone \%wt } \\
\text { non-washing }\end{array}$ & $\begin{array}{l}\text { Time } \\
\text { hr }\end{array}$ & $\begin{array}{l}0.00086 \quad \mathrm{~N} \quad \mathrm{Na}_{2} \mathrm{~S}_{2} \mathrm{O}_{3} \\
\text { Titration } \\
\text { consumption } \mathrm{ml}\end{array}$ & $\begin{array}{l}\text { Area of } \\
\text { samples } \mathrm{m}^{2}\end{array}$ & $\begin{array}{c}\text { Peroxide at } \\
0.1 \mathrm{~g} / \mathrm{L} \mathrm{Cu}{ }^{2+} \\
\text { catalyst } \\
\mathrm{mmol} / \mathrm{m}^{2}\end{array}$ & $\begin{array}{l}\mathrm{In} \mathrm{DW} \\
\mathrm{mmol} / \mathrm{m}^{2}\end{array}$ \\
\hline 3.0 & $\begin{array}{c}0.25 \\
\text { hr }\end{array}$ & 4. 1 & 0.003468 & 0.5083 & 0.4340 \\
\hline 3.0 & $0.5 \mathrm{hr}$ & 5.8 & 0.003468 & 0.7191 & 0.6943 \\
\hline 3.0 & $\begin{array}{c}0.75 \\
\mathrm{hr}\end{array}$ & 8.7 & 0.003468 & 1.0787 & 0.9919 \\
\hline
\end{tabular}




\section{Appendix XII}

\section{Experimental Data Tables of catalyst test of PPMM reaction with ozone}

Table XII-2 Peroxide data table of PPMM in aqueous medium at different concentration of $\mathrm{Cu}^{2+}$ catalyst (with $\mathrm{CuSO}_{4} \cdot 5 \mathrm{H}_{2} \mathrm{O}$ )

The PPMM sample pieces were fixed in reactor and treated by aqueous ozonation.

The reactor was filled with $10 \mathrm{~L}$ distilled water and different concentration of $\mathrm{Cu}^{2+}$ catalyst was added (with $\mathrm{CuSO}_{4} \cdot 5 \mathrm{H}_{2} \mathrm{O}$ ), respectively.

Ozone flow was in $10 \mathrm{~L} / \mathrm{min}$ at concentration of $3.0 \mathrm{wt} \%$ and pressure of $15 \mathrm{psig}$, ozone bubbled in water. Ozonation time was $30 \mathrm{~min}$.

Ozonated samples were dried and degassed by vacuum in more than $1.5 \mathrm{hrs}$.

The standard iodometric titration was applied to test the peroxide concentration.

\begin{tabular}{|c|c|c|c|c|c|c|c|c|c|}
\hline $\begin{array}{c}\text { Reaction } \\
\text { time }\end{array}$ & Ozone & $\begin{array}{c}0.025 \mathrm{~g} / \mathrm{L} \\
\mathrm{Cu}^{2+}\end{array}$ & & $\begin{array}{c}0.05 \mathrm{~g} / \mathrm{L} \\
\mathrm{Cu}^{2}\end{array}$ & & $\begin{array}{c}0.10 \mathrm{~g} / \mathrm{L} \\
\mathrm{Cu}^{2}\end{array}$ & & $\begin{array}{c}0.20 \mathrm{~g} / \mathrm{L} \\
\mathrm{Cu}^{2}\end{array}$ & \\
\hline & & $\begin{array}{c}0.787 \\
\mathrm{mmol} / \mathrm{L}\end{array}$ & & $\begin{array}{c}1.574 \\
\mathrm{mmol} / \mathrm{L}\end{array}$ & & $\begin{array}{c}3.148 \\
\mathrm{mmol} / \mathrm{L}\end{array}$ & & $\begin{array}{c}6.296 \\
\mathrm{mmol} / \mathrm{L}\end{array}$ & \\
\hline minutes & $3.0 \mathrm{wt} \%$ & $8.4 \times 10^{-4} \mathrm{~N}$ & $\begin{array}{c}\mathrm{OOH} \\
\mathrm{CONC}\end{array}$ & $\begin{array}{c}8.4 \times 10^{-4} \\
\mathrm{~N}\end{array}$ & $\begin{array}{c}\mathrm{OOH} \\
\mathrm{CONC}\end{array}$ & $8.4 \times 10^{-4} \mathrm{~N}$ & $\begin{array}{c}\mathrm{OOH} \\
\mathrm{CONC}\end{array}$ & $8.4 \times 10^{-4} \mathrm{~N}$ & $\begin{array}{c}\mathrm{OOH} \\
\mathrm{CONC}\end{array}$ \\
\hline & & $\mathrm{ml}$ & $\mathrm{mmol} / \mathrm{M}^{2}$ & $\mathrm{ml}$ & $\mathrm{mmol} / \mathrm{M}^{2}$ & $\mathrm{ml}$ & $\mathrm{mmol} / \mathrm{M}^{2}$ & $\mathrm{ml}$ & $\mathrm{mmol} / \mathrm{M}^{2}$ \\
\hline 30 & & 11.7 & 0.7253 & 11.9 & 0.7377 & 11.6 & 0.7191 & 11.3 & 0.7005 \\
\hline $\begin{array}{c}\text { Reaction } \\
\text { time }\end{array}$ & Ozone & DW & & & & & & & \\
\hline minutes & $3.0 \mathrm{wt} \%$ & $\begin{array}{c}8.4 \times 10^{-4} \\
\mathrm{~N}\end{array}$ & $\begin{array}{c}\mathrm{OOH} \\
\mathrm{CONC}\end{array}$ & & & & & & \\
\hline & & $\mathrm{ml}$ & $\mathrm{mmol} / \mathrm{M}^{2}$ & & & & & & \\
\hline 30 & & 11.2 & 0.6943 & & & & & & \\
\hline
\end{tabular}




\section{Appendix XII}

\section{Experimental Data Tables of catalyst test of PPMM reaction with ozone}

Table XII-3 Scavenger's $\left(\mathrm{Na}_{2} \mathrm{CO}_{3}\right)$ effect on the aqueous ozonation and $\mathrm{Cu}^{2+}$ catalytic ozonation

The PPMM sample pieces were fixed in reactor and treated by aqueous ozonation.

The reactor was filled with $10 \mathrm{~L}$ distilled water and $1.574 \mathrm{mmol} / \mathrm{L} \mathrm{Cu}^{2+}$ catalyst was added (with $\mathrm{CuSO}_{4} \cdot 5 \mathrm{H}_{2} \mathrm{O}$ ). Then scavenger of $3.0 \mathrm{~g} / \mathrm{L}$ carbonate $\left(\mathrm{Na}_{2} \mathrm{CO}_{3}\right)$ was added to the solution.

Ozone flow was in $10 \mathrm{~L} / \mathrm{min}$ at concentration of $3.0 \mathrm{wt} \%$ and pressure of $15 \mathrm{psig}$, ozone bubbled in water.

Ozonated samples were dried and degassed by vacuum in more than $1.5 \mathrm{hrs}$.

The standard iodometric titration was applied to test the peroxide concentration.

\begin{tabular}{|c|c|c|c|c|c|c|c|}
\hline \multirow{2}{*}{ Ozone \%wt } & \multirow[b]{2}{*}{ Time hr } & \multicolumn{3}{|c|}{$\begin{array}{c}0.00086 \mathrm{~N} \mathrm{Na}_{2} \mathrm{~S}_{2} \mathrm{O}_{3} \text { Titration } \\
\text { consumption ml }\end{array}$} & \multirow{2}{*}{ 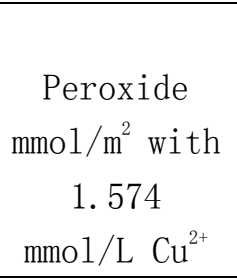 } & \multirow{2}{*}{$\begin{array}{c}\text { Peroxide } \\
\mathrm{mmol} / \mathrm{m}^{2} \text { with } \\
\mathrm{Cu}^{2+}+3.0 \mathrm{~g} / \mathrm{L} \\
\text { Scavenger } \\
\mathrm{CO}_{3}{ }^{2+}\end{array}$} & \multirow{2}{*}{$\begin{array}{l}\text { Peroxide } \\
\mathrm{mmol} / \mathrm{m}^{2} \text { in } \\
\text { aqueous } \\
\text { 3. 0wt\% } \mathrm{O}_{3}\end{array}$} \\
\hline & & $\begin{array}{c}1.574 \\
\mathrm{mmol} / \mathrm{L} \\
\mathrm{Cu}^{2+}\end{array}$ & $\begin{array}{c}\mathrm{Cu}^{2+}+ \\
\text { Scavenge } \\
\mathrm{r} \mathrm{CO}_{3}{ }^{2+}\end{array}$ & $\begin{array}{c}\text { 3. } 0 \mathrm{wt} \% \\
\text { aqueous } \mathrm{O}_{3} \\
\text { AVG }\end{array}$ & & & \\
\hline 3.0 & 0.25 & 3.8 & 3.0 & 3.0 & 0.5259 & 0.4083 & 0.4092 \\
\hline 3.0 & 0.5 & 5.6 & 4.3 & 4.5 & 0.7751 & 0.5951 & 0.6235 \\
\hline 3.0 & 0.75 & 7.2 & 5.5 & 6.5 & 0.9965 & 0.7612 & 0.8995 \\
\hline
\end{tabular}




\section{Appendix XII}

\section{Experimental Data Tables of catalyst test of PPMM reaction with ozone}

Table XII-4 Scavenger's (t-Butanol) effect on the aqueous ozonation and $\mathrm{Cu}^{2+}$ catalytic ozonation

The PPMM sample pieces were fixed in reactor and treated by aqueous ozonation.

The reactor was filled with $10 \mathrm{~L}$ distilled water and $1.574 \mathrm{mmol} / \mathrm{L} \mathrm{Cu}^{2+}$ catalyst was added (with $\mathrm{CuSO}_{4} \cdot 5 \mathrm{H}_{2} \mathrm{O}$ ). Then scavenger of $0.1 \mathrm{~mol} / \mathrm{L} \mathrm{t}$-Butanol was added to the solution.

Ozone flow was in $10 \mathrm{~L} / \mathrm{min}$ at concentration of $3.0 \mathrm{wt} \%$ and pressure of $15 \mathrm{psig}$, ozone bubbled in water.

Ozonated samples were dried and degassed by vacuum in more than $1.5 \mathrm{hrs}$.

The standard iodometric titration was applied to test the peroxide concentration.

\begin{tabular}{|c|c|c|c|c|c|c|c|}
\hline \multirow[b]{2}{*}{ Ozone \%wt } & \multirow[b]{2}{*}{ Time hr } & \multicolumn{3}{|c|}{$\begin{array}{l}0.00086 \mathrm{~N} \mathrm{Na}_{2} \mathrm{~S}_{2} \mathrm{O}_{3} \text { Titration } \\
\text { consumption } \mathrm{ml}\end{array}$} & \multirow{2}{*}{$\begin{array}{c}\text { Peroxide } \\
\mathrm{mmol} / \mathrm{m}^{2} \\
\text { with } \\
1.574 \\
\mathrm{mmol} / \mathrm{L} \\
\mathrm{Cu}^{2+}\end{array}$} & \multirow{2}{*}{$\begin{array}{c}\text { Peroxide } \\
\mathrm{mmol} / \mathrm{m}^{2} \\
\text { with } \mathrm{Cu}^{2+}+ \\
3.0 \mathrm{~g} / \mathrm{L} \\
\text { Scavenger } \\
\mathrm{CO}_{3}{ }^{2+}\end{array}$} & \multirow[b]{2}{*}{$\begin{array}{c}\text { Peroxide } \\
\mathrm{mmol} / \mathrm{m}^{2} \text { in } \\
\mathrm{Cu}^{2+}+0.1 \\
\text { mol } \\
\text { T-butanol }\end{array}$} \\
\hline & & $\begin{array}{c}1.574 \\
\mathrm{mmol} / \mathrm{L} \\
\mathrm{Cu}^{2+}\end{array}$ & $\begin{array}{c}\mathrm{Cu}^{2+}+ \\
\text { Scaveng } \\
\text { er } \mathrm{CO}_{3}{ }^{2+}\end{array}$ & $\begin{array}{c}\mathrm{Cu}^{2+}+0.1 \\
\text { mol } \\
\mathrm{T}-\text { butano } \\
1\end{array}$ & & & \\
\hline 3.0 & 0.25 & 3.8 & 3.0 & 2.7 & 0.5259 & 0.4083 & 0.3270 \\
\hline 3.0 & 0.5 & 5.6 & 4.3 & 4.8 & 0.7751 & 0.5951 & 0.5813 \\
\hline 3.0 & 0.75 & 7.2 & 5.5 & 6.3 & 0.9965 & 0.7612 & 0.7629 \\
\hline
\end{tabular}




\section{Appendix XII}

\section{Experimental Data Tables of catalyst test of PPMM reaction with ozone}

Table XII-5 The effect of scavengers on $\mathrm{Cu}^{2+}$ catalytic ozonation (with $\mathrm{CuSO}_{4} \mathbf{5 H}_{2} \mathrm{O}$ ) -

$$
3.0 \mathrm{~g} / \mathrm{L} \mathrm{Na}_{2} \mathrm{CO}_{3}-\text { in cold water }\left({ }^{\circ} \mathrm{C}\right)
$$

The PPMM sample pieces were fixed in reactor and treated by aqueous ozonation.

The reactor was filled with $10 \mathrm{~L}$ cold distilled water $\left(0{ }^{\circ} \mathrm{C}\right)$ and $1.574 \mathrm{mmol} / \mathrm{L} \mathrm{Cu}^{2+}$ catalyst was added (with $\mathrm{CuSO}_{4} \cdot 5 \mathrm{H}_{2} \mathrm{O}$ ). Then scavenger of $3.0 \mathrm{~g} / \mathrm{L}$ carbonate $\left(\mathrm{Na}_{2} \mathrm{CO}_{3}\right)$ was added to the solution.

Ozone flow was in $10 \mathrm{~L} / \mathrm{min}$ at concentration of $3.0 \mathrm{wt} \%$ and pressure of $15 \mathrm{psig}$, ozone bubbled in water.

Ozonated samples were dried and degassed by vacuum in more than $1.5 \mathrm{hrs}$.

The standard iodometric titration was applied to test the peroxide concentration.

\begin{tabular}{|c|c|c|c|c|c|c|c|}
\hline \multirow[t]{2}{*}{ Ozone \%wt } & \multirow{2}{*}{$\begin{array}{l}\text { Time } \\
\text { hr }\end{array}$} & \multicolumn{3}{|c|}{$\begin{array}{lll}0.00086 \quad \mathrm{~N} \mathrm{Na}_{2} \mathrm{~S}_{2} \mathrm{O}_{3} & \text { Titration } \\
\text { consumption } \mathrm{ml} & \end{array}$} & \multicolumn{3}{|c|}{ Peroxide $\mathrm{mmol} / \mathrm{m}^{2}$} \\
\hline & & $\mathrm{DW}\left(0{ }^{\circ} \mathrm{C}\right)$ & $\begin{array}{r}\mathrm{Cu}^{2+}+ \\
\mathrm{DW}\left(0^{\circ} \mathrm{C}\right)\end{array}$ & $\begin{array}{c}\mathrm{Cu}{ }^{2+}+ \\
\mathrm{CO}_{3}^{2-}\left(0{ }^{\circ} \mathrm{C}\right)\end{array}$ & $\mathrm{DW}\left(0{ }^{\circ} \mathrm{C}\right)$ & $\begin{array}{c}\mathrm{Cu}^{2+} \\
\mathrm{DW}\left(0^{\circ} \mathrm{C}\right)\end{array}$ & $\begin{array}{c}\mathrm{Cu}{ }^{2+}+ \\
\mathrm{CO}_{3}{ }^{2-}\left(0{ }^{\circ} \mathrm{C}\right)\end{array}$ \\
\hline 3.0 & $\begin{array}{l}0.25 \\
\mathrm{hr}\end{array}$ & 1.2 & 1.5 & 1. 1 & 0.1661 & 0.2076 & 0.1522 \\
\hline 3.0 & $0.5 \mathrm{hr}$ & 1.9 & 2.45 & 1.5 & 0.2630 & 0.3391 & 0.2076 \\
\hline 3.0 & $\begin{array}{l}0.75 \\
\text { hr }\end{array}$ & 2.5 & 3.1 & 1.8 & 0.3460 & 0.4290 & 0.2491 \\
\hline
\end{tabular}




\section{Appendix XII}

\section{Experimental Data Tables of catalyst test of PPMM reaction with ozone}

Table XII-6 $\mathrm{Na}_{2} \mathrm{SO}_{4}$ applied for 30 minutes at $3.0 \mathrm{wt} \%$ DW ozonation

The PPMM sample pieces were fixed in reactor and treated by aqueous ozonation.

The reactor was filled with $10 \mathrm{~L}$ distilled water and different concentration of anion $\mathrm{SO}_{4}{ }^{2-}$ was added (with $\mathrm{Na}_{2} \mathrm{SO}_{4}$ ), respectively.

Ozone flow was in $10 \mathrm{~L} / \mathrm{min}$ at concentration of $3.0 \mathrm{wt} \%$ and pressure of $15 \mathrm{psig}$, ozone bubbled in water. Ozonation time was $30 \mathrm{~min}$.

Ozonated samples were dried and degassed by vacuum in more than $1.5 \mathrm{hrs}$.

The standard iodometric titration was applied to test the peroxide concentration.

\begin{tabular}{|c|c|c|c|c|c|c|c|}
\hline \multirow{2}{*}{ Ozone \%wt } & \multirow[b]{2}{*}{$\begin{array}{l}\text { Time } \\
\text { hr }\end{array}$} & \multicolumn{3}{|c|}{$\begin{array}{l}0.00086 \mathrm{~N} \mathrm{Na} \mathrm{S}_{2} \mathrm{O}_{3} \text { Titration } \\
\text { consumption } \mathrm{ml}\end{array}$} & \multicolumn{3}{|c|}{ Peroxide $\mathrm{mmol} / \mathrm{m}^{2}$} \\
\hline & & $\begin{array}{c}0.0005 \\
\mathrm{~mol} / \mathrm{L} \\
\mathrm{SO}_{4}{ }^{2-}\end{array}$ & $\begin{array}{c}0.005 \\
\mathrm{~mol} / \mathrm{L} \\
\mathrm{SO}_{4}^{2-}\end{array}$ & $\begin{array}{c}0.05 \\
\mathrm{~mol} / \mathrm{L} \\
\mathrm{SO}_{4}^{2-}\end{array}$ & $\begin{array}{c}0.0005 \\
\mathrm{~mol} / \mathrm{L} \\
\mathrm{SO}_{4}{ }^{2-}\end{array}$ & $\begin{array}{c}0.005 \\
\mathrm{~mol} / \mathrm{L} \\
\mathrm{SO}_{4}^{2-}\end{array}$ & $\begin{array}{c}0.05 \\
\mathrm{~mol} / \mathrm{L} \\
\mathrm{SO}_{4}{ }^{2-} \\
\end{array}$ \\
\hline 3.0 & $\begin{array}{l}0.25 \\
\text { hr }\end{array}$ & 3.4 & 3.2 & 3 & 0.4117 & 0.3875 & 0.3633 \\
\hline 3.0 & $0.5 \mathrm{hr}$ & 5.6 & 5.4 & 5 & 0.6782 & 0.6540 & 0.6055 \\
\hline 3.0 & $\begin{array}{l}0.75 \\
\mathrm{hr}\end{array}$ & 7.9 & 7 & 6.3 & 0.9567 & 0.8477 & 0.7629 \\
\hline
\end{tabular}




\section{Appendix XII}

\section{Experimental Data Tables of catalyst test of PPMM reaction with ozone}

Table XII-7 $\mathrm{Na}_{2} \mathrm{SO}_{4}$ applied for 15 - and 30-minute intervals to 3.0 wt\% $\mathrm{O}_{3}$ and $\mathbf{0 . 7 8 6 8}$ $\mathrm{mmol} / \mathrm{L} \mathrm{Cu}^{2+}$ catalytic ozonation

The PPMM sample pieces were fixed in reactor and treated by aqueous ozonation.

The reactor was filled with $10 \mathrm{~L}$ distilled water and $0.7868 \mathrm{mmol} / \mathrm{L} \mathrm{Cu}^{2+}$ catalyst, then different concentration of anion $\mathrm{SO}_{4}{ }^{2-}$ was added (with $\mathrm{Na}_{2} \mathrm{SO}_{4}$ ), respectively.

Ozone flow was in $10 \mathrm{~L} / \mathrm{min}$ at concentration of $3.0 \mathrm{wt} \%$ and pressure of $15 \mathrm{psig}$, ozone bubbled in water.

Ozonated samples were dried and degassed by vacuum in more than $1.5 \mathrm{hrs}$.

The standard iodometric titration was applied to test the peroxide concentration.

\begin{tabular}{|c|c|c|c|c|c|c|c|}
\hline \multirow{2}{*}{ Ozone \%wt } & \multirow[b]{2}{*}{$\begin{array}{l}\text { Time } \\
\text { hr }\end{array}$} & \multicolumn{3}{|c|}{$\begin{array}{l}0.00086 \mathrm{~N} \mathrm{Na} \mathrm{S}_{2} \mathrm{O}_{3} \text { Titration } \\
\text { consumption } \mathrm{ml}\end{array}$} & \multicolumn{3}{|c|}{ Peroxide $\mathrm{mmol} / \mathrm{m}^{2}$} \\
\hline & & $\begin{array}{c}0.000 \\
\mathrm{~mol} / \mathrm{L} \\
\mathrm{SO}_{4}{ }^{2-}\end{array}$ & $\begin{array}{c}0.005 \\
\mathrm{~mol} / \mathrm{L} \\
\mathrm{SO}_{4}{ }^{2-}\end{array}$ & $\begin{array}{c}0.05 \\
\mathrm{~mol} / \mathrm{L} \\
\mathrm{SO}_{4}{ }^{2-}\end{array}$ & $\begin{array}{c}0.000 \\
\mathrm{~mol} / \mathrm{L} \\
\mathrm{SO}_{4}^{2-}\end{array}$ & $\begin{array}{c}0.005 \\
\mathrm{~mol} / \mathrm{L} \\
\mathrm{SO}_{4}{ }^{2-}\end{array}$ & $\begin{array}{c}0.05 \\
\mathrm{~mol} / \mathrm{L} \\
\mathrm{SO}_{4}{ }^{2-}\end{array}$ \\
\hline 3.0 & $\begin{array}{l}0.25 \\
h r\end{array}$ & 4 & 3.9 & 3.8 & 0.4844 & 0.4723 & 0.4602 \\
\hline 3.0 & $0.5 \mathrm{hr}$ & 6.4 & 5.9 & 5.4 & 0.7751 & 0.7145 & 0.6540 \\
\hline
\end{tabular}




\section{Appendix XII}

\section{Experimental Data Tables of catalyst test of PPMM reaction with ozone}

Table XII-8 Different concentrations of $\mathrm{Cu}^{2+}$ catalyst applied to cold water $\left(0^{\circ} \mathrm{C}\right)$ ozonation

The PPMM sample pieces were fixed in reactor and treated by aqueous ozonation.

The reactor was filled with $10 \mathrm{~L}$ cold distilled water $\left(0^{\circ} \mathrm{C}\right)$, and different concentration of $\mathrm{Cu}^{2+}$ catalyst was added, respectively.

Ozone flow was in $10 \mathrm{~L} / \mathrm{min}$ at concentration of $3.0 \mathrm{wt} \%$ and pressure of $15 \mathrm{psig}$, ozone bubbled in water.

Ozonated samples were dried and degassed by vacuum in more than $1.5 \mathrm{hrs}$.

The standard iodometric titration was applied to test the peroxide concentration.

\begin{tabular}{|c|c|c|c|c|c|c|}
\hline $\mathrm{Cu}^{2+} \mathrm{mmol} / \mathrm{L}$ & 0.157 & 0.383 & 0.787 & 1.574 & 3.934 & $0.0-\mathrm{DW}$ \\
\hline $\begin{array}{c}\text { Ozonation } \\
\text { time }\end{array}$ & \multicolumn{6}{|c|}{$0.00086 \mathrm{~N} \mathrm{Na}_{2} \mathrm{~S}_{2} \mathrm{O}_{3}$ Titration consumption $\mathrm{ml}$} \\
\hline $15 \min$ & 1.3 & 1.5 & 1.71 & 1.7 & 1.4 & 1.3 \\
\hline $30 \min$ & 2.2 & 2.6 & 2.8 & 2.7 & 2.3 & 2.2 \\
\hline $\mathrm{Cu}^{2+} \mathrm{mmol} / \mathrm{L}$ & 0.157 & 0.383 & 0.787 & 1.574 & 3.934 & $0.0-\mathrm{DW}$ \\
\hline $\begin{array}{c}\text { Ozonation } \\
\text { time }\end{array}$ & \multicolumn{6}{|c|}{ Peroxide concentration mmol/L } \\
\hline $15 \min$ & 0.1574 & 0.1817 & 0.2076 & 0.2059 & 0.1695 & 0.1661 \\
\hline $30 \min$ & 0.2664 & 0.3149 & 0.3391 & 0.3270 & 0.2785 & 0.2630 \\
\hline
\end{tabular}




\section{Appendix XII}

\section{Experimental Data Tables of catalyst test of PPMM reaction with ozone}

Table XII-9 Detection of NB capture ${ }^{\circ} \mathrm{OH}$ in $\mathrm{Cu}^{2+}$ catalytic ozonation by $410 \mathrm{~nm}$ UV-Vis absorption

The PPMM sample pieces were fixed in reactor and treated by $\mathrm{Cu}^{2+}$ catalytic ozonation.

$75 \mathrm{ml}$ of the ozonation solution was taken out in certain interval of reaction time, and mixed into $25 \mathrm{ml}$ Nitrobenzene (NB) immediately. Shake for $5 \mathrm{~min}$. The water was removed, and the reacted NB was tested by UV-Vis in $410 \mathrm{~nm}$ wave length.

\begin{tabular}{|l|c|c|c|c|}
\hline $\begin{array}{l}\text { Ozonation } \\
\text { time-min }\end{array}$ & 0 & 3 & 13 & 20 \\
\hline $\begin{array}{l}\text { UV-Vis } \\
\text { adsorption } \\
\text { Index -1 }\end{array}$ & 1.714 & 2.011 & 1.886 & 1.877 \\
\hline $\begin{array}{l}\text { UV-Vis } \\
\text { adsorption } \\
\text { Index -2 }\end{array}$ & 2.02 & 1.831 & 1.98 & 1.996 \\
\hline $\begin{array}{l}\text { UV-Vis } \\
\text { adsorption } \\
\text { Index -Avg }\end{array}$ & 1.867 & 1.921 & 1.933 & 1.9365 \\
\hline
\end{tabular}




\section{Appendix XII}

\section{Experimental Data Tables of catalyst test of PPMM reaction with ozone}

Table XII-10 Detection of NB capture ${ }^{\circ} \mathrm{OH}$ in pure water ozonation by $410 \mathrm{~nm}$ UV-Vis absorption

The PPMM sample pieces were fixed in reactor and treated by aqueous ozonation without catalyst.

$75 \mathrm{ml}$ of the ozonation solution was taken out in different interval of reaction time, respectively, and mixed into $25 \mathrm{ml}$ Nitrobenzene (NB) immediately. Shake for $5 \mathrm{~min}$. The water was removed, and the reacted NB was tested by UV-Vis in $410 \mathrm{~nm}$ wave length.

\begin{tabular}{|l|c|c|c|c|}
\hline $\begin{array}{l}\text { Ozonation } \\
\text { time-min }\end{array}$ & 0 & 3 & 13 & 20 \\
\hline $\begin{array}{l}\text { UV-Vis } \\
\text { adsorption } \\
\text { Index-1 }\end{array}$ & 1.72 & 1.721 & 1.936 & 1.726 \\
\hline $\begin{array}{l}\text { UV-Vis } \\
\text { adsorption } \\
\text { Index-2 }\end{array}$ & 1.982 & 1.987 & 1.779 & 2.026 \\
\hline $\begin{array}{l}\text { UV-Vis } \\
\text { adsorption } \\
\text { Index- Avg }\end{array}$ & 1.851 & 1.854 & 1.8575 & $1 . .876$ \\
\hline
\end{tabular}




\section{Appendix XII}

\section{Experimental Data Tables of catalyst test of PPMM reaction with ozone}

Table XII-11 Peroxide data table of PPMM in aqueous medium at different concentration of $\mathrm{Fe}^{3+}$ catalyst

The PPMM sample pieces were fixed in reactor and treated by aqueous ozonation.

The reactor was filled with $10 \mathrm{~L}$ distilled water, and different concentration of $\mathrm{Fe}^{3+}$ catalyst (with $\mathrm{FeCl}_{3} \cdot 6 \mathrm{H}_{2} \mathrm{O}$ ) was added, respectively.

Ozone flow was in $10 \mathrm{~L} / \mathrm{min}$ at concentration of $3.0 \mathrm{wt} \%$ and pressure of $15 \mathrm{psig}$, ozone bubbled in water.

Ozonated samples were dried and degassed by vacuum in more than $1.5 \mathrm{hrs}$.

The standard iodometric titration was applied to test the peroxide concentration.

\begin{tabular}{|c|c|c|c|c|c|c|c|c|c|}
\hline $\begin{array}{c}\text { Reaction } \\
\text { time }\end{array}$ & Ozone & $\begin{array}{l}0.0616 \\
\mathrm{mmol} / \\
\mathrm{L} \mathrm{Fe}^{3+}\end{array}$ & & $\begin{array}{c}0.3696 \\
\mathrm{mmol} / \mathrm{L} \\
\mathrm{Fe}^{3+}\end{array}$ & & $\begin{array}{c}0.616 \\
\mathrm{mmol} / \mathrm{L} \\
\mathrm{Fe}^{3+}\end{array}$ & & $\begin{array}{c}3.6959 \\
\mathrm{mmol} / \mathrm{L} \\
\mathrm{Fe}^{3+}\end{array}$ & \\
\hline \multirow[t]{2}{*}{ minutes } & $3.0 \mathrm{wt} \%$ & $\begin{array}{c}8.4 \times 10^{-4} \\
\mathrm{~N}\end{array}$ & $\begin{array}{c}\text { Peroxide } \\
\text { CONC }\end{array}$ & $\begin{array}{c}8.4 \times 10^{-4} \\
\mathrm{~N}\end{array}$ & $\begin{array}{c}\text { Peroxide } \\
\text { CONC }\end{array}$ & $\begin{array}{c}8.4 \times 10^{-4} \\
\mathrm{~N}\end{array}$ & $\begin{array}{c}\text { Peroxide } \\
\text { CONC }\end{array}$ & $\begin{array}{c}8.4 \times 10^{-4} \\
\mathrm{~N}\end{array}$ & $\begin{array}{c}\text { Peroxide } \\
\text { CONC }\end{array}$ \\
\hline & & $\mathrm{ml}$ & $\mathrm{mmol} / \mathrm{M}^{2}$ & $\mathrm{ml}$ & $\mathrm{mmol} / \mathrm{M}^{2}$ & $\mathrm{ml}$ & $\mathrm{mmol} / \mathrm{M}^{2}$ & $\mathrm{ml}$ & $\mathrm{mmol} / \mathrm{M}^{2}$ \\
\hline 30 & $\begin{array}{c}\text { Sample } \\
1\end{array}$ & 13.1 & 0.8121 & 13.5 & 0.8369 & 13.6 & 0.8431 & 12.8 & 0.7935 \\
\hline \multicolumn{2}{|l|}{ DW } & & & & & & & & \\
\hline $8.4 \times 10^{-4} \mathrm{~N}$ & $\begin{array}{l}\text { Peroxid } \\
\text { e CONC }\end{array}$ & & & & & & & & \\
\hline $\mathrm{ml}$ & $\mathrm{mmol} / \mathrm{M}^{2}$ & & & & & & & & \\
\hline 11.6 & 0.7191 & & & & & & & & \\
\hline
\end{tabular}




\section{Appendix XIII}

\section{Experimental Data Tables of BOPP reaction with ozone}

\section{Table XIII-1 Peroxide data table of BOPP in gaseous phase}

The BOPP sample pieces were fixed in reactor and treated by gaseous ozonation.

Ozone flow was in $10 \mathrm{~L} / \mathrm{min}$ at concentration of $3.0 \mathrm{wt} \%$ and pressure of $15 \mathrm{psig}$.

Ozonated samples were degassed by vacuum in more than $1.5 \mathrm{hrs}$.

The standard iodometric titration was applied to test the peroxide concentration.

\begin{tabular}{|c|c|c|c|c|c|c|c|}
\hline \multirow{2}{*}{$\begin{array}{c}\text { Ozone } \\
\text { \%wt }\end{array}$} & $\begin{array}{c}\text { Time } \\
\text { hr }\end{array}$ & $\begin{array}{l}\text { Titration } \\
\text { consumption ml }\end{array}$ & \multicolumn{2}{c|}{$\begin{array}{c}\text { Peroxide } \\
\text { Concentration } \\
\text { mmol/m }\end{array}$} & $\begin{array}{c}\text { Average } \\
\mathrm{mmol} / \mathrm{m}^{2}\end{array}$ & Error +/- \\
\cline { 2 - 8 } & & $\begin{array}{c}\text { Sample } \\
1\end{array}$ & $\begin{array}{c}\text { Sample } \\
2\end{array}$ & Sample 1 & Sample 2 & & \\
\hline 3.0 & 0.25 & 3.4 & 3.5 & 0.0705 & 0.0748 & 0.0726 & 0.00214 \\
\hline 3.0 & 0.50 & 5.45 & 5.1 & 0.124 & 0.109 & 0.116 & 0.0118 \\
\hline 3.0 & 0.75 & 6.65 & 6.4 & 0.147 & 0.137 & 0.142 & 0.000 \\
\hline 3.0 & 1.00 & 8.64 & 7.8 & 0.185 & 0.167 & 0.176 & 0.0032 \\
\hline 3.0 & 1.25 & 10.04 & 9.6 & 0.215 & 0.205 & 0.210 & 0.0082 \\
\hline AVG & & & & & & & \\
\hline
\end{tabular}




\section{Appendix XIII}

\section{Experimental Data Tables of BOPP reaction with ozone}

Table XIII-2 Peroxide data table of BOPP in aqueous phase

The BOPP sample pieces were fixed in reactor and treated by aqueous ozonation.

The reactor was filled with $10 \mathrm{~L}$ distilled water.

Ozone flow was in $10 \mathrm{~L} / \mathrm{min}$ at concentration of $3.0 \mathrm{wt} \%$ and pressure of $15 \mathrm{psig}$, ozone bubbled in water.

Ozonated samples were dried and degassed by vacuum in more than $1.5 \mathrm{hrs}$.

The standard iodometric titration was applied to test the peroxide concentration.

\begin{tabular}{|c|c|c|c|c|c|c|c|}
\hline \multirow{2}{*}{$\begin{array}{c}\text { Ozone } \\
\text { \%wt }\end{array}$} & \multirow{2}{*}{$\begin{array}{l}\text { Time } \\
\text { hr }\end{array}$} & $\begin{array}{l}\text { l.00086 } \\
\text { Titration } \\
\text { consumption } \mathrm{ml}\end{array}$ & \multicolumn{2}{|c|}{$\begin{array}{l}\text { Peroxide } \\
\text { Concentration mmol/m }\end{array}$} & $\begin{array}{l}\text { Average } \\
\mathrm{mmol} / \mathrm{m}^{2} \mathrm{O}_{3}\end{array}$ & $\begin{array}{c}\text { Error } \\
+/-\end{array}$ \\
\cline { 3 - 8 } & Sample 1 & Sample 2 & Sample 1 & Sample 2 & & \\
\hline 3.0 & 0.25 & 3.2 & 3.4 & 0.726 & 0.684 & 0.071 & 0.00213 \\
\hline 3.0 & 0.50 & 4.7 & 5.8 & 0.124 & 0.100 & 0.112 & 0.00748 \\
\hline 3.0 & 0.75 & 6.4 & 6.5 & 0.138 & 0.139 & 0.139 & 0.00534 \\
\hline 3.0 & 1.00 & 8.4 & 8.2 & 0.180 & 0.174 & 0.177 & 0.00855 \\
\hline 3.0 & 1.25 & 8.8 & 9.3 & 0.188 & 0.1986 & 0.1933 & 0.00535 \\
\hline AVG & & & & & & & \\
\hline
\end{tabular}




\section{Appendix XIV}

\section{Experimental Data Tables of peroxide concentration aging of BOPP}

\section{Table XIV peroxide concentration aging of BOPP}

The BOPP sample pieces were fixed in reactor and treated by aqueous ozonation.

The dried and degassed samples were tested by the standard iodometric titration in different time.

\begin{tabular}{|c|c|c|c|c|c|c|c|c|c|}
\hline $\begin{array}{l}\text { Reaction } \\
\text { time }\end{array}$ & Ozone & 1 day & & 3 days & & 2 weeks & & 4 weeks & \\
\hline minutes & $3.0 \mathrm{wt} \%$ & $\begin{array}{c}8.6 \\
\times 10^{-4} \mathrm{~N}\end{array}$ & $\begin{array}{c}\mathrm{OOH} \\
\mathrm{CONC}\end{array}$ & $\begin{array}{c}8.6 \\
\times 10^{-4} \mathrm{~N}\end{array}$ & $\begin{array}{c}\mathrm{OOH} \\
\mathrm{CONC}\end{array}$ & $\begin{array}{c}8.6 \times 10^{-4} \\
\mathrm{~N}\end{array}$ & $\begin{array}{c}\mathrm{OOH} \\
\mathrm{CONC}\end{array}$ & $\begin{array}{c}8.6 \times 10^{-4} \\
\mathrm{~N}\end{array}$ & $\begin{array}{c}\mathrm{OOH} \\
\mathrm{CONC}\end{array}$ \\
\hline & & $\mathrm{ml}$ & $\mathrm{mmol} / \mathrm{M}^{2}$ & $\mathrm{ml}$ & $\mathrm{mmol} / \mathrm{M}^{2}$ & $\mathrm{ml}$ & $\mathrm{mmol} / \mathrm{M}^{2}$ & $\mathrm{ml}$ & $\mathrm{mmol} / \mathrm{M}^{2}$ \\
\hline 60 & & 9.4 & 0.0047 & 9 & 0.0045 & 7 & 0.0035 & 5.5 & 0.00275 \\
\hline$\%$ & & & 100 & & 95.74 & & 74.47 & & 58.51 \\
\hline
\end{tabular}




\section{Appendix XV}

\section{Experimental Data Tables of BOPP aqueous ozonation with scavengers}

Table XV Peroxide data table of BOPP in aqueous medium with scavenger- Sodium Carbonate Concentration $3 \mathrm{~g} / \mathrm{L}$

The BOPP sample pieces were fixed in reactor and treated by aqueous ozonation.

The reactor was filled with $10 \mathrm{~L}$ distilled water and with scavenger- Sodium Carbonate dissolved in.

Ozone flow was in $10 \mathrm{~L} / \mathrm{min}$ at concentration of $3.0 \mathrm{wt} \%$ and pressure of $15 \mathrm{psig}$, ozone bubbled in water.

Ozonated samples were dried and degassed by vacuum in more than $1.5 \mathrm{hrs}$.

The standard iodometric titration was applied to test the peroxide concentration.

\begin{tabular}{|c|c|c|c|c|c|c|c|}
\hline \multirow{2}{*}{$\begin{array}{c}\text { Ozone } \\
\% w t\end{array}$} & \multirow{2}{*}{$\begin{array}{l}\text { Time } \\
\text { minutes }\end{array}$} & \multicolumn{2}{|c|}{$\begin{array}{l}0.00084 \quad \mathrm{~N} \quad \mathrm{Na}_{2} \mathrm{~S}_{2} \mathrm{O}_{3} \\
\text { Titration } \\
\text { consumption } \mathrm{ml}\end{array}$} & \multicolumn{2}{|c|}{$\begin{array}{c}\text { Peroxide } \\
\text { Concentration } \\
\mathrm{mmol} / \mathrm{m}^{2} \\
\end{array}$} & \multirow{2}{*}{$\begin{array}{l}\text { Average } \\
\mathrm{mmol} / \mathrm{m}^{2}\end{array}$} & \multirow[t]{2}{*}{$\begin{array}{c}\text { Error } \\
+/-\end{array}$} \\
\hline & & $\begin{array}{c}\text { Sample } \\
1 \\
\end{array}$ & $\begin{array}{c}\text { Sample } \\
2 \\
\end{array}$ & $\begin{array}{c}\text { Sample } \\
1 \\
\end{array}$ & $\begin{array}{c}\text { Sample } \\
2 \\
\end{array}$ & & \\
\hline 3.0 & 15 & 2.4 & 2.3 & 0.05586 & 0.05524 & 0.05535 & 0.00119 \\
\hline 3.0 & 30 & 3.7 & 3.5 & 0.08905 & 0.08419 & 0.08662 & 0.00121 \\
\hline 3.0 & 45 & 5.0 & 4.6 & 0.1197 & 0.1098 & 0.1148 & 0.00357 \\
\hline 3.0 & 60 & 6.2 & 6.3 & 0.1485 & 0.1491 & 0.1488 & 0.00358 \\
\hline 3.0 & 75 & 6.9 & 7.2 & 0.1646 & 0.1710 & 0.1678 & 0.00476 \\
\hline $\mathrm{AVG}$ & & & & & & & \\
\hline
\end{tabular}




\section{Appendix XVI}

\section{Experimental Data Tables of washing test of BOPP reaction with ozone}

Table XVI Peroxide data table of BOPP in gaseous medium with/without 10\% IPA + distilled water washing 15 minutes

The BOPP sample pieces were fixed in reactor and treated by gaseous ozone. Ozone flow was in $10 \mathrm{~L} / \mathrm{min}$ at concentration of $3.0 \mathrm{wt} \%$ and pressure of $15 \mathrm{psig}$.

Ozonated samples were split in half, one half was washed by $10 \%$ IPA + distilled water, and another half was not washed. Then dried and degassed by vacuum in more than $1.5 \mathrm{hrs}$.

The standard iodometric titration was applied to test the peroxide concentration.

\begin{tabular}{|c|c|c|c|c|c|c|c|c|c|}
\hline \multirow{3}{*}{$\begin{array}{l}\text { Ozone } \\
\text { \%wt }\end{array}$} & \multirow{3}{*}{$\begin{array}{l}\text { Time } \\
\text { hr }\end{array}$} & \multicolumn{3}{|c|}{$\begin{array}{llr}0.00095 & \mathrm{~N} & \mathrm{Na}_{2} \mathrm{~S}_{2} \mathrm{O}_{3} \\
\text { Titration } & \text { consumption } \\
\mathrm{ml} & & \end{array}$} & \multicolumn{3}{|c|}{$\begin{array}{l}\text { Peroxide Concentration } \\
\qquad \mathrm{mmol} / \mathrm{m}^{2}\end{array}$} & \multirow{3}{*}{$\begin{array}{l}\text { Average } \\
\mathrm{mmol} / \mathrm{m}^{2} \\
\text { Washed }\end{array}$} & \multirow{3}{*}{$\begin{array}{c}\text { Error } \\
+/- \\
\text { Washed }\end{array}$} \\
\hline & & \multicolumn{2}{|c|}{ Washed } & $\begin{array}{l}\text { Non- } \\
\text { washed }\end{array}$ & \multicolumn{2}{|c|}{ Washed } & $\begin{array}{l}\text { Non- } \\
\text { washed }\end{array}$ & & \\
\hline & & $\begin{array}{c}\text { Sample } \\
1\end{array}$ & $\begin{array}{c}\text { Sample } \\
2\end{array}$ & $\begin{array}{c}\text { Sample } \\
1\end{array}$ & $\begin{array}{c}\text { Sample } \\
1\end{array}$ & $\begin{array}{c}\text { Sample } \\
2\end{array}$ & $\begin{array}{c}\text { Sample } \\
1\end{array}$ & & \\
\hline 3.0 & 0.25 & 2.2 & 2.3 & 2.5 & 0.052 & 0.0679 & 0.060 & 0.0536 & 0.0012 \\
\hline 3.0 & 0.5 & 3.6 & 3.5 & 4 & 0.086 & 0.0893 & 0.095 & 0.0845 & 0.0012 \\
\hline 3.0 & 0.75 & 4.7 & 5 & 5.2 & 0.112 & 0.1245 & 0.124 & 0.1155 & 0.0036 \\
\hline $\mathrm{AVG}$ & & & & & & & & & 0.0020 \\
\hline
\end{tabular}




\section{Appendix XVI}

\section{Experimental Data Tables of washing test of BOPP reaction with ozone}

Table XVI-2 Peroxide data table of BOPP in aqueous medium with/without $10 \%$ IPA + distilled water washing 15 minutes

The BOPP sample pieces were fixed in reactor and treated by aqueous ozonation. Ozone flow was in $10 \mathrm{~L} / \mathrm{min}$ at concentration of $3.0 \mathrm{wt} \%$ and pressure of $15 \mathrm{psig}$. Ozone bubbled in water.

Ozonated samples were split in half, one half was washed by $10 \%$ IPA + distilled water, and another half was not washed. Then dried and degassed by vacuum in more than $1.5 \mathrm{hrs}$.

The standard iodometric titration was applied to test the peroxide concentration.

\begin{tabular}{|c|c|c|c|c|c|c|c|c|c|}
\hline \multirow{3}{*}{$\begin{array}{l}\text { Ozone } \\
\text { \%wt }\end{array}$} & \multirow{3}{*}{$\begin{array}{l}\text { Time } \\
\text { hr }\end{array}$} & \multicolumn{3}{|c|}{$\begin{array}{l}0.00095 \mathrm{~N} \mathrm{Na}_{2} \mathrm{~S}_{2} \mathrm{O}_{3} \text { Titration } \\
\text { consumption } \mathrm{ml}\end{array}$} & \multicolumn{3}{|c|}{$\begin{array}{l}\text { Peroxide Concentration } \\
\qquad \mathrm{mmol} / \mathrm{m}^{2}\end{array}$} & \multirow{3}{*}{$\begin{array}{l}\text { Washed } \\
\text { average } \\
\mathrm{mmol} / \mathrm{m}^{2}\end{array}$} & \multirow{3}{*}{$\begin{array}{c}\text { Washed } \\
\text { Error } \\
+/-\end{array}$} \\
\hline & & \multicolumn{2}{|c|}{ washed } & \multirow{2}{*}{$\begin{array}{l}\text { Non- } \\
\text { washing } \\
\text { Sample } \\
1\end{array}$} & \multicolumn{2}{|c|}{ washed } & \multirow{2}{*}{$\begin{array}{c}\begin{array}{l}\text { Non- } \\
\text { washing }\end{array} \\
\text { Sample } \\
1\end{array}$} & & \\
\hline & & $\begin{array}{c}\text { Sample } \\
1 \\
\end{array}$ & $\begin{array}{l}\text { Sample } \\
\quad 2 \\
\end{array}$ & & $\begin{array}{c}\text { Sample } \\
1 \\
\end{array}$ & $\begin{array}{c}\text { Sample } \\
2 \\
\end{array}$ & & & \\
\hline 3.0 & 0.25 & 3 & 2.85 & 2.95 & 0.071 & 0.0679 & 0.070 & 0.0690 & 0.0012 \\
\hline 3.0 & 0.5 & 3.85 & 3.75 & 3.95 & 0.092 & 0.0893 & 0.094 & 0.0917 & 0.0024 \\
\hline 3.0 & 0.75 & 5.05 & 5.23 & 5.25 & 0.120 & 0.1245 & 0.125 & 0.1248 & 0.0002 \\
\hline $\mathrm{AVG}$ & & & & & & & & & 0.0013 \\
\hline
\end{tabular}




\section{Appendix XVII}

\section{Experimental Data Tables of different $\mathrm{pH}$ test of BOPP reaction with ozone}

Table XVII Peroxide data table of BOPP in aqueous medium at different pH (3.0wt\% $\mathrm{O}_{3}$ and 30 minutes ozonation time

The BOPP sample pieces were fixed in reactor and treated by aqueous ozonation.

The reactor was filled with $10 \mathrm{~L}$ distilled water and set different $\mathrm{pH}$, respectively, adjusted by $1.0 \mathrm{M} \mathrm{H}_{2} \mathrm{SO}_{4}$ and $1.0 \mathrm{M} \mathrm{NaOH}$.

Ozone flow was in $10 \mathrm{~L} / \mathrm{min}$ at concentration of $3.0 \mathrm{wt} \%$ and pressure of $15 \mathrm{psig}$, ozone bubbled in water. Ozonation time is $30 \mathrm{~min}$.

Ozonated samples were dried and degassed by vacuum in more than $1.5 \mathrm{hrs}$.

The standard iodometric titration was applied to test the peroxide concentration.

\begin{tabular}{|c|c|c|c|c|c|c|c|}
\hline \multirow{2}{*}{$\begin{array}{c}\text { Ozone } \\
\% w t\end{array}$} & \multirow[t]{2}{*}{$\mathrm{pH}$} & \multicolumn{2}{|c|}{$\begin{array}{llr}0.00084 & \mathrm{~N} & \mathrm{Na}_{2} \mathrm{~S}_{2} \mathrm{O}_{3} \\
\text { Titration } & \text { consumption } \\
\text { m] }\end{array}$} & \multicolumn{2}{|c|}{$\begin{array}{l}\text { Peroxide Concentration } \\
\qquad \mathrm{mmol} / \mathrm{m}^{2}\end{array}$} & \multirow{2}{*}{$\begin{array}{l}\text { Average } \\
\mathrm{mmol} / \mathrm{m}^{2}\end{array}$} & \multirow{2}{*}{$\begin{array}{c}\text { Error } \\
+/-\end{array}$} \\
\hline & & $\begin{array}{c}\text { Sample } \\
1\end{array}$ & $\begin{array}{c}\text { Sample } \\
2\end{array}$ & $\begin{array}{c}\text { Sample } \\
1\end{array}$ & $\begin{array}{c}\text { Sample } \\
2\end{array}$ & & \\
\hline 3.0 & 3 & 3.8 & 3.6 & 0.0905 & 0.0857 & 0.0881 & 0.0024 \\
\hline 3.0 & 4 & 3.9 & 4 & 0.0929 & 0.0952 & 0.0940 & 0.0012 \\
\hline 3.0 & 5 & 3.9 & 3.8 & 0.0929 & 0.0905 & 0.0917 & 0.0012 \\
\hline 3.0 & 6 & 3.4 & 3.3 & 0.0810 & 0.0786 & 0.0798 & 0.0012 \\
\hline 3.0 & 7 & 3.5 & 3.3 & 0.0833 & 0.0786 & 0.0810 & 0.0024 \\
\hline 3.0 & 8 & 3.2 & 3.3 & 0.0762 & 0.0786 & 0.0774 & 0.0012 \\
\hline 3.0 & 9 & 3.2 & 3.3 & 0.0762 & 0.0786 & 0.0774 & 0.0012 \\
\hline AVG & & & & & & & 0.0015 \\
\hline
\end{tabular}




\section{Appendix XVIII}

\section{Experimental Data Tables of catalyst test of BOPP reaction with ozone}

Table XVIII Peroxide data table of BOPP in aqueous medium at $0.06884 \mathrm{~g} / \mathrm{L} \mathrm{Fe}^{3+}$ catalyst (with $\mathrm{FeCl}_{3} \cdot 6 \mathrm{H}_{2} \mathrm{O}$ )

The BOPP sample pieces were fixed in reactor and treated by aqueous ozonation.

The reactor was filled with $10 \mathrm{~L}$ distilled water and $0.06884 \mathrm{~g} / \mathrm{L} \mathrm{Fe}^{3+}$ catalyst (with $\left.\mathrm{FeCl}_{3} \cdot 6 \mathrm{H}_{2} \mathrm{O}\right)$

was added. Ozone flow was in $10 \mathrm{~L} / \mathrm{min}$ at concentration of $3.0 \mathrm{wt} \%$ and pressure of $15 \mathrm{psig}$, ozone bubbled in water.

Ozonated samples were dried and degassed by vacuum in more than $1.5 \mathrm{hrs}$.

The standard iodometric titration was applied to test the peroxide concentration.

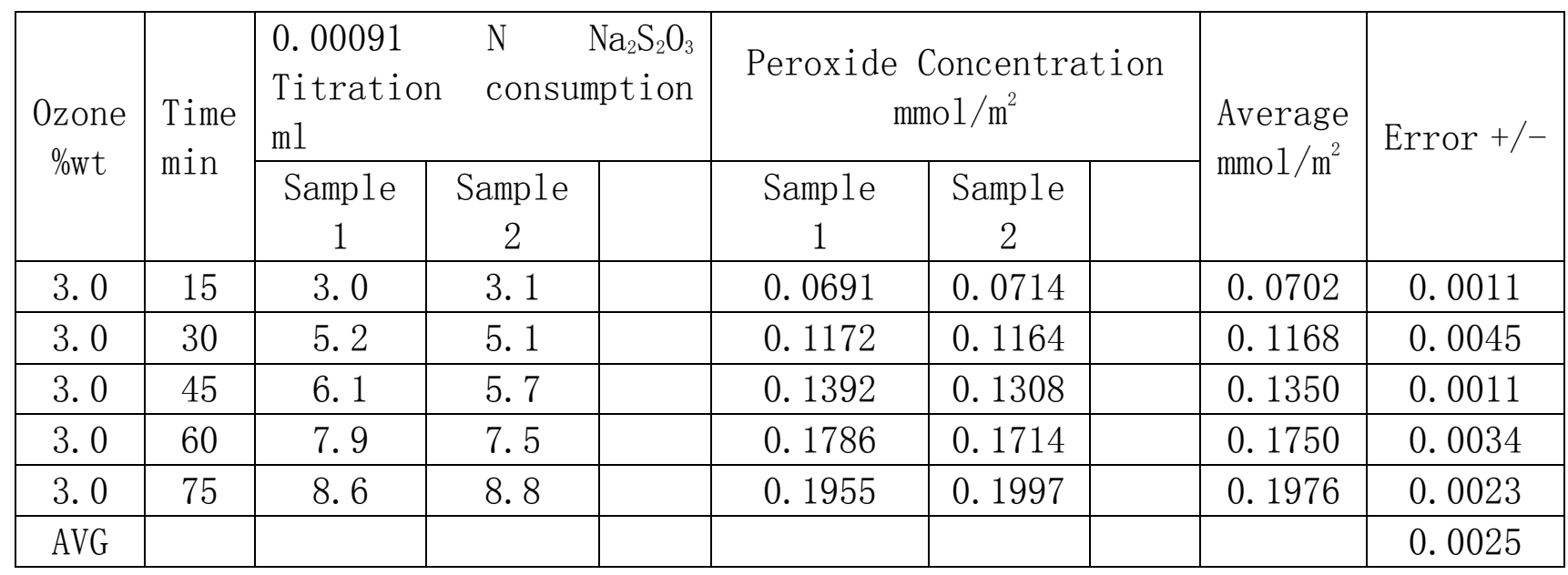




\section{References}

Belfer S., Purinson Y., Fainshtein R., Radchenko Y., Kedem O., Surface modification for commercial composite polyamide reverse osmosis membranes, J. Membrane Sci. 139, 1998, 175-181.

Beltran F., Theoretical aspects of the kinetics of competitive ozone reactions in water, Ozone Sci. Eng., 17, 1995, 163-181.

Beltran F., Ozone Reaction Kinetic for Water and Wastewater Systems, Lewis Publishers, a CRC Press Company, New York, USA, 2004.

Boutevin B., Robin J., Torres N., Casteil J., Graft copolymerization of styrene onto ozonized polyethylene, Macromol. Chem. Phys. 2002, 245-252.

Bruice P., Organic Chemistry, Prentice Hall, Upper Saddle River, NJ 2004.

Buhler R., Staehelin J., Hoign. B J., Ozone decomposition in water studied by pulse radiolysis. $1 . \mathrm{HO}_{2} / \mathrm{O}_{2}{ }^{-}$and $\mathrm{HO}_{3} / \mathrm{O}_{3}{ }^{-}$as intermediates, J. Phys. Chem. 88, 1984, 2560-2564.

Calderbank P. H., Lewis J. M. O., Ozone-decomposition Catalysis, Chem. Eng. Sci., Vol. 31, Pergamon Press, printed in Great Britain, 1976, 1216.

Callister, Jr. W. D., Material Science and Engineering, An Introduction, $16^{\text {th }}$ edition, John Wiley \& Sons, Inc, printed in USA, 2003.

Chang Y., Shih Y., Ruaan R., Higuchi A., Chenb W., Lai J., Preparation of poly (vinylidene fluoride) microfiltration membrane with uniform surface-copolymerized poly (ethylene glycol) methacrylate and improvement of blood compatibility, J. Mem. Sci., 309, 2008, $165-174$.

Cheng F., Hong S., Ho C., The Adhesion properties of an ozone modified thermoplastic olefin elastomer, J. Adhesion, 67, 1998, 123-137.

Davidson M., Mitchell S., Bradley R., UV/ozone modification of plasma-polymerized acetonnitrille films for enhanced cell attachment, J. Col Surf b, 01, 2004, 008.

Dasgupta, S., Surface modification of polyolefin for hydrophilicity and bondabiity : \

Ozonization and grafting hydrophilic monomers on ozonized polyolefin, J. Appl. Polym. Sci., 41, 1990, 233-248. 
Ershov B. G., Morozov P. A., Decomposition of ozone in water at pH 4-8, Russian J. of Appl. Chem., 81. No.11. 2008, pp. 1895-1898.

Escobar I., Hock E., Committee Report: Recent advances and research needs in membrane fouling, American Water Works Association. J. 97.8, ABI/INFORM Trade \& Industry, 2005,79 .

Feng J., Aki S., Chateauneuf J. and Brennecke J., Experimental Determination of Hydroxyl Radical Reactivity in Supercritical Water Using Pulse Radiolysis Department of Chemical Engineering, University of Notre Dame, Notre Dame, IN-46556; Department of Chemistry, Wester Michigan University, Kalamazoo, MI-49008, 2011.

Fornl L., Bahnemann D., Edwln J. Hart, Mechanism of the hydroxide ion initiated decomposition of ozone in aqueous solution, J. Phys. Chem. 86, 1982, 255-259.

Fujimoto K., Takebayashi Y., Inoue H., Ikada Y., Ozone-induced graft polymerization onto polymer surface J. Polym. Sci. 31, 1993, 1035.

Gabelman A., Hwang S., Hollow fiber membrane contactors, J. Membr. Sci. 159, 1999, 61-106.

Gatenholm P., Ashida T., Hoffman A., J. Polym. Sci. A: Polym. Chem. 35, 1996, 461.

Hill G. R., Kinetics, Mechanism, and Activation Energy of the Cobaltous Ion Catalyzed Decomposition of Ozone, contribution no. 77 from the chemical laboratory of the University of Utah, 70, 1948, 1306-1307.

Gottschalk C., Libra J. A., Saupe A., Ozonation of Water and waste Water, A practical Guide to Understanding Ozone and its Application,WILEY-VCH Verlag GmbH, D-69469 Weinheim, Germany, 2000.

Gu, H. B., Hydrophilicity improvement of selected films by ozonation and graft polymerization, Chem. Eng. Department, Ryerson University, 2008.

Gu, H. B., Wu, J., Doan H., Hydrophilicity enhancement of high density polyethylene film ozonation, Chem. Eng. Technol., 32, N0. 5, 2009, 726-731.

Gurol, M.D. Singer, P.C., Kinetics of ozone decomposition: a dynamic approach, Environ. Sci. Technol., 16, no. 7, 1982, 377-383.

Hewes C. G., Davison R. R., Kinetics of ozone decomposition reaction with organics in water, AlChe Journal, 17, No. 1, 1971, 141-147. 
Hoigné J., Bader H., Rate constants of reactions of ozone with organic and inorganic compounds in water III: inorganic compounds and radicals, Wat. Res. 19, 1985, 993.

Hu M., Wan L., Liu Z., Dai Z., Xu Z., Fabrication of glycosylated surfaces on microporous polypropylene membranes for protein recognition and adsorption, J. Mater. Chem., 2008, 18.

Hua H., Li N., Wu L., Zhong H., Tang L., Anti-fouling ultrafiltration membrane prepared from polysultone-graft-methyl acrylate copolymers by UV-induced grafting method, J. Environ. Sci. 20, 2008, 565-570.

Kai T., Tsurum T., Nakao S.-I., Kimura S., Preparation of hollow-fiber membranes by plasma-graft filling polymerization for organic-liquid separation, J. Membrane. Sci. 170, 2000, 61-70.

Kang G., Liu M., A novel method of surface modification on thin-film composite reverse osmosis membrane by grafting poly(ethylene glycol), Polymer 48, 2007, 1165-1170.

Kasprzyk-Hordern B., Ziolek M., Nawrocki J., Catalytic ozonation and methods of enhancing molecular ozone reactions in water treatment, Applied Catalysis B: Environmental 46, 2003, 639-669.

Kefeli, A. A., Razumovskii, S. D., Zaikov, G. Y., Interaction of polyethylene with ozone, Polym. Sci. U.S.S.R, 13, 1971, 904-911.

Kilduff J., Mattaraj S., Sensibaugh J., Pieracci J., Yuan Y. \& Belfort G., Modeling flux decline during nanofiltration of NOM with poly(arylsulfone) membranes modified using UV-assisted graft polymerization, Environ. Eng. Sci. 19, 2002, 477-495.

Kilduff J., Mattaraj S., Zhou M., Belfort G., Kinetics of membrane flux decline: the role of natural colloids and mitigation via membrane surface modification, J. Nanoparticle Res. 7, $2005,525-544$.

Kim M., Saito K., Furusaki S., Water flux and protein adsorption of a hollow fiber modified with hydroxyl groups, J. Mem. Sci. 56, 1991, 289-302.

Kim M., Saito K., Radiation-induced graft polymerization and sulfonation of glycidyl methacrylate on to porous hollow-fiber membranes with different pore sizes, Radiat. Phys. Chem. 57, 2000, 167-172. 
Kiyohara S., Kim M., Toida Y., Saito K., Sugita K., Sugo T., Selection of a precursor monomer for the introduction of affinity ligands onto a porous membrane by radiationinduced graft polymerization, J. Chromatogr. A 758, 1997, 209-215.

Kokatnur V. R., Jelling M., Indometric determination of peroxygen in organic compound, J. Am. Chem. Soc. 63, 1941, 1432-1433.

Ku Y., Su W. J., Shen Y. S., Ind. Eng. Chem. Res., 35, no. 10, 1996, 3369-3374.

Kulik E., Ivanchenko M., Kato K., Sano S., and Ikada Y., Peroxide Generation and Decomposition on Polymer Surface, J. Polym. Sci.: Part A Polymer Chemistry, 33, 1995, 323-330.

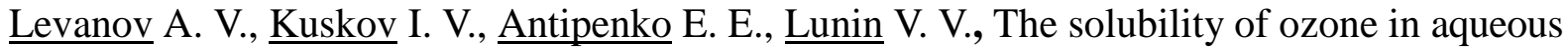
solutions of sulfuric, phosphoric, and perchloric acids, Russian J. Phys. Chem. A, Focus on Chemistry, 82, No\# 7, 2008, 1126-1131.

Logager T., Holcman J., Sehested K., Pedersen T., Oxidation of ferrous ions by ozone in acidic solution, Inorg. Chem. 31, 1992, 3523-3529.

Low S., Cheng L., Lee S., Water softening using a generic low cost nano-filtration membrane, Desalination 221, 2008, 168-173.

L'opez A., L'opez, J. S. Pic, Benbelkacem H., Debellefontaine H., Influence of $t$-butanol and of $\mathrm{pH}$ on hydrodynamic and mass transfer parameters in an ozonation process, Chemical Engineering and Processing 46, 2007, 649-655.

Ma H., Bowman C., Davis R., Membrane fouling reduction by backpulsing and surface modification, J. Membrane. Sci. 173, 2000, 191-200.

Markee T., Kelsey Prihoda K., Mays N., Cangelosi A., STANDARD OPERATING PROCEDURE-Procedure for Analyzing the Concentration of Ozone in Water, Procedure No: GSI/SOP/BS/RA/C/1, July 10, 2009, 1-5.

Mastan E., Wu J., Doan H., An investigation into surface modification of polyethylene films for hydrophilicity enhancement by catalytic ozonation, J. Appl. Polym. Sci. 128, Issue 1, $2013,828-835$.

Max S. Peters, Klaus D Timmerhaus, Ronald E. West, Plant design and economics for chemical engineers, fifth edition, the McGraw-Hill Companies, Inc, 2003. 
Michel V., C. Marzin, Tarrago G., Durand J., New membranes bearing pyridinic ligands by plasma graft polymerization, J. Appl. Polym. Sci. 70, 1998, 359-366.

Michael M. N., El-Zaher N. A., Ibrahim S. F., Polymer-Plastics Technology and Engineering, 43, no. 4, 2004, 1041-1052.

Morris J. C., The Aqueous Solubility of Ozone-A Review, Ozone News, No.1, 1988, 14-16.

Murakami T., Fukushima Y., Hirano Y., Tokuoka Y., Takahashi M., Kawashima N., Modification of PS films by combined treatment of ozone aeration and UV irradiation in aqueous ammonia solution for the introduction of amine and amide groups on their surface, Appl. Surf. Sci. 249, 2005, 425.

Murakami, T. N., Fushinori, Y., Hirano, Y., Tokouka, Y., Takahashi, M., Kawashima, N., Surface modification of polystyrene and poly (methyl methacrylate) by active oxygen treatment, Colloid Surface B: Biointerfaces 29, 2003, 171-179.

Nuffield E. N., X-ray diffraction methods, New York, Wiley, 1966

Patel Dipak P., An investigation into surface modificatin of polyethylene film by ozonation. Thesis (M.A.Sc.), Ryerson University, 2008.

Phattaranawika J., Leiknesa T., Pronk W., Mass transfer studies in flat-sheet membrane contactor with ozonation, J. Mem. Sci. 247, 2005, 153-167.

Portaccio M., El-Masry M., An amperometric sensor employing glucose oxidase immobilized on nylon membranes with different pore diameter and grafted with different monomers, J. Mol. Cat. B: Enzymatic 18, 2002, 49-67.

Razumovskii S. D., Kefeli A. A., Zaikov G. E., Degradation of Polymers in reactive gases, Eur. Polym. J., 7, 1971, 275-285.

Robin, J., The use of ozone in the synthesis of new polymers and the modification of polymers, Adv

Polym Sci . 167, 2004, 35-79.

Roth J., Sullivan D., Solubility of ozone in water, Indust. Eng. Chem. Fundam. 20 , 1981, $137-140$.

Saito N., Yamashita S., Characterization of surfacecharge-mosaic-modified ultrafiltration membranes prepared by laser-induced surface graft polymerization, J. Appl. Polym. Sci. 67, 1998, 1141-1149. 
Sainbayar A., Kim J. S., Jung W. J., Lee Y. S., Lee C. H., Application of Surface Modified Polypropylene Membranes to an Anaerobic Membrane Bioreactor, Environ. Technol., 22, 2001, 1035-1042.

Sayed E., Hegazy A., Abdel-Rehim H., Characterization and application of radiation grafted membranes in waste water treatment, Czechoslovak J. Physics, 50, 2000, No. 2.

Sharma R., Sims R. A., Mazumder M. K., Modification of Surface Properties of Polymeric Materials, J. the Arkansas Academy of Sci., 56, 2002, 157-161.

Sonntag C. V., Gunten U.V., Chemistry of Ozone in Water and Wastewater Treatment for Basic Principles to Applications, IWA Publishing, London, UK, 2012, 13-14.

Sotelo, J. L. et al., Ozone decomposition in water: kinetic study, Ind. Eng. Chem. Res., 26, 1987, 39-43.

Staehelln J., Hoigné J., Decomposition of Ozone in Water: Rate of Initiation by Hydroxide Ions and Hydrogen Peroxide, Environ. Sci. Technol. 16, 1982, 676-681.

Taube H., Bray W. C., Chain Reactions in Aqueous Solutions Containing Ozone, Hydrogen Peroxide and Acid, Chain Reactions in Aqueous Solutions, Contribution from the Chemical Laboratory of the University of California, Berkley, Calif , Dec., 1940, 3357-3373.

Taniguchi M., Belfort G., Low protein fouling synthetic membranes by UV-assisted surface grafting modification: varying monomer type, J. Membrane Sci. 231, 2004, 147-157.

Teramoto M., Imamura S., Yatagai N., et al., J. Chem. Eng. Jpn., 14, no. 5, 1981, 383-388.

Turmanova S., Radiation grafting of Acrylic acid onto Polytetrafluoroethylene film for glucose oxidase immobilization and its application in membrane biosensor, J. Membrane Sci., 127, 1997, 1-7.

Tu C., Liu Y., Lee K., Lai J., Hydrophilic surface-grafted poly(tetrafluoroethylene) membranes using in pervaporation dehydration processes, J. Membrane. Sci. 274, 2006, 47.

Ulbricht M., Belfort G., Surface modification of ultrafiltration membranes by low temperature plasma. II. Graft polymerization onto polyacrylonitrile and polysulfone, J. Membrane Sci. 111, 1996, 193-215. 
Ulbricht M., Yang H., Porous Polypropylene Membranes with Different Carboxyl Polymer Brush Layers for Reversible Protein Binding via Surface-Initiated Graft Copolymerization, Chem Materials, 17, 2005, 2622-2631.

Wang Y., Kim J., Choo K., Lee Y., Lee C., Hydrophilic modification of polypropylene microfiltration membranes by ozone-induced graft polymerization, J. Membrane Sci. 169, 2000, 269-276.

Wavhal D.S., Fisher E.R., Hydrophilic modification of polyethersulfone membranes by low temperature plasmainduced graft polymerization, J. Membrane Sci. 209, 2002, 255-269.

Wu J. Rudy K. Spark J. Oxidation of aqueous phenol by ozone and peroxidase, Advances in Environ. Res, 4, 2000, 339-346.

Xua P., Drewesa J., Kimb T., Bellona C., Amy G., Effect of membrane fouling on transport of organic contaminants in NF/RO membrane applications, J. Membrane Sci. 279, 2006, $165-175$.

Yamagishi H., Crivello J., Belfort G., Development of a novel photochemical technique for modifying poly (arylsulfone) ultrafiltration membranes, J. Membrane Sci. 105, 1995a, $237-247$.

Yong K., Wu J., Andrews S., Heterogeneous Catalytic Ozonation of Aqueous Reactive Dye, Ozone Sci. Eng. 27, 2005, 257.

Yuan Y., AI F., Zhang J., Zhang X., Shen J. and Lin S., Grafting sulfobetaine monomer onto the segmented poly (ether-urethane) surface to improve hemocompatibility, Biomater. Sci. Polymer Edn, 13, 2002, 1081-1092.

Yuan Y., Zhang J., Ai F., Yuan J., Zhou J., Shen J., Lin S., Surface modification of SPEU films by ozone induced graft copolymerization to improve hemocompatibility, Colloids Surf. B: Biointerfaces 29(4), 2003, 247.

Yu H., Liu L., Tang Z., Yan M., Gu J., Wei X., Mitigated membrane fouling in an SMBR by surface modification, J. Membrane Sci. 310, 2008, 409-417.

Yu H., Xie Y., Hu M., Wang J., Wang S., Xu Z., Surface modification of polypropylene microporous membrane to improve its antifouling property in MBR: $\mathrm{CO} 2$ plasma treatment, J. Membrane. Sci. 254, 2005, 219-227. 
Feng J, Sudhir N. V. K. Aki, John E. Chateauneuf and Joan F. Brennecke*, Experimental Determination of Hydroxyl Radical Reactivity in Supercritical Water Using Pulse Radiolysis, http://www.isasf.net/fileadmin/files/Docs/Versailles/Papers/PRw6.pdf, Aug, 2013.

Gullinkala T., Escobar I., Study of the Hydrophilic-Enhanced Ultrafiltration Membrane, Wiley InterScience (www.interscience.wiley.com), published online 22 April, 2008.

Membrane technology, Lenntech membrane fouling, http://www.lenntech.com/ membrane-fouling.htm, 2009.

Morao A., Escobar I., Pessoa de Amorim M., Lopes A., and Goncalvesa I., Postsynthesis Modification of a Cellulose Acetate Ultrafiltration Membrane for Applications in Water and Wastewater Treatment, Wiley InterScience (www.interscience.wiley.com), Published online 17 November 2005 .

Ozone solubility, http://www.ozoneapplications.com/info/ozone_properties.htm, Oct, 2010.

Schematic of membrane fouling, http://www.lenntech.com/membrane-fouling.htm, Oct, 2008. 